\title{
I. UNDERWAY GEOPHYSICAL DATA: GLOMAR CHALLENGER LEG 43
}

\author{
Brian E. Tucholke, Lamont-Doherty Geological Observatory of Columbia University, Palisades, New York
}

\section{INTRODUCTION}

Bathymetric, magnetic, and seismic profiler data were acquired during underway operations on Glomar Challenger Cruise 43 between Istanbul, Turkey, and Norfolk, Virginia (13 June to 12 August 1975). These data are presented in two groups of profiles, the first containing bathymetric and magnetic data (Figure 1), and the second group showing reproductions of the seismic profiler records (Figure 2). A map in the back cover pocket shows the Leg 43 cruise track annotated with day and hour (Greenwich Mean Time or Z) and with distance along track in hundreds of nautical miles (filled triangles); bathymetric contours on this map are at intervals of 2000 corrected meters from Uchupi (1971) and Defense Mapping Agency (1972).

Leg 43 navigation data are listed in Table 1 together with distance along track (nautical miles), and speed and course maintained between navigation points. Regional magnetic-field values computed from the coefficients of Cain et al. (1968) are listed at the right.

The methods of data reduction and display in Figure 1 are described by Talwani (1969). In each profile are listed (from top to bottom) date, time (Z), and crossings of integral latitudes and longitudes. The upper (lighter) profile gives the bathymetric data at a vertical exaggeration of $100: 1$; the depth scale $(D)$ is in nominal fathoms (1/400 sec two-way travel time). The lower, darker profile shows the magnetic anomaly values in gammas (scale $M$ ); the regional magnetic field has been removed using the IGRF coefficients of Cain et al. (1968). At the bottom of these profiles is listed the distance (linear scale) along the cruise track in nautical miles, and immediately above are ticks showing the position of each navigation point. Selected navigation points are annotated with latitude, longitude, and speed (knots) and course maintained between navigation points. The only navigation points annotated are those where speed changed by more than one knot and/or ship's course varied by more than five degrees.

The seismic reflection profiles (Figure 2) are shown as a function of time, and they run from left to right, top to bottom on the page. The depth scales are annotated in seconds reflection time ( 1 second $=400 \mathrm{fm}$ nominal). Assuming sediment velocities of $2 \mathrm{~km} / \mathrm{sec}$, depths below sea floor in seconds equal sediment thicknesses in kilometers. Along the profiles, time in days and hours $(Z)$ is annotated at the bottom. Course and speed made good at any point along these profiles can be found in Table 1. Distance along track in hundreds of nautical miles, drill site locations, and physiographic information are annotated in the upper part of the profiles. The seismic profiling system used to obtain these records consisted of 10 in. $^{3}$ and 40 in. $^{3}$ Bolt airguns fired simultaneously, a Scripps-designed hydrophone array, Bolt amplifiers, two bandpass filters, and two EDO dry-paper recorders. Recordings normally were made at a 5-sec sweep and a 10-sec sweep; only the better of these records is reproduced in Figure 2. Filter settings vary, and they are annotated on the records (for example, F 40/160 $=40-160 \mathrm{~Hz}$ bandpass).

The $12-\mathrm{kHz}$ profiles were recorded on a Gifft precision depth recorder and were used to read water depths. These profiles rarely showed any sub-bottom penetration. On Leg $43,3.5 \mathrm{kHz}$ profiles were not recorded.

\section{NARRATIVE}

The Glomar Challenger profile departing from Istanbul passes through the Sea of Marmara; only echosounding data were recorded as the ship proceeded through the Dardanelles. The first thick sediments recorded by the seismic profiler are in the Cretan Basin, where more than 1 second (nominal $1 \mathrm{~km}$ ) of weakly deformed sediments is present. Deformation becomes much stronger and faulting is observed in the Hellenic Arc and Trench farther west.

West of the Hellenic Trench the Mediterranean Ridge and the Messina Cone both exhibit very irregular sea floor with acoustically nonlaminated or weakly laminated sediments overlying a strong smooth reflector. This horizon is "Reflector $M$ " which marks the top of the upper Miocene evaporite sequence in the Mediterranean (Ryan, Hsü, et al., 1973). The irregular sea floor has been interpreted as "karst topography" resulting from solution of the underlying salt (Hsü et al., 1973).

The Challenger profile continues through the Straits of Sicily where irregular sub-bottom reflectors commonly are covered by ponded stratified sediments. Profiles recorded over the Balearic Abyssal Plain show that the acoustically laminated turbidites are underlain by a weakly laminated interval and by Reflector $M$. Salt diapirism is apparent in several locations beneath the abyssal plain. Reflector $M$ continues into the Alboran Basin_beneath a nonlaminated acoustic interval; finely laminated sediments overlying these sediments continue upward to the sea floor. Only bathymetric data were recorded as the Challenger passed through the Straits of Gibraltar.

In the eastern North Atlantic the Challenger profile cuts across the northeast corner of the Horseshoe Abyssal Plain before crossing Gorringe and Josephine 
Banks. A shallow, nearly flat top is apparent on Josephine Bank. Thin unconsolidated sediments which are acoustically nonlaminated to weakly laminated cover most of the banks as well as the lower flanks of the Azores Rise to the west.

Sedimentary reflectors become much stronger along the profile near the Azores, showing the influx of coarse clastic debris from the islands. The western Azores Plateau exhibits a relatively smooth acoustic basement with overlying, moderately thick sediments ( $\simeq 800 \mathrm{~m}$ ). In sharp contrast, the crest of the Mid-Atlantic Ridge is rugged and sediment free except for local ponding of carbonate oozes. On the west flank of the Mid-Atlantic Ridge, the Challenger profile cuts into the Pico Fracture Zone at a very shallow angle and follows the trend of the fracture zone west to the Sohm Abyssal Plain. Because the Challenger profile crosses a variety of anomalous features in passing over the MidAtlantic Ridge, it is very difficult to identify any specific magnetic anomalies along the track.

The sediments of the Sohm Abyssal Plain slope westward from the Mid-Atlantic Ridge flank to about $49^{\circ} \mathrm{W}$ where Challenger changed course toward the southwest, headed for Nashville Seamount. The general southward slope of the central Sohm Abyssal Plain is then apparent in the profile approaching Nashville Seamount and in the profile between Sites 383 and 382. The high reflectivity of the abyssal plain sediments generally prevents recognition and identification of individual deep reflectors. Curiously, reflectors in the turbidites seem to be better resolved in the central part of the abyssal plain, near Site 383.

North and northeast of Nashville Seamount (Site 382 ) sizable deposits of acoustically nonlaminated sediments crop out or are locally capped by the laminated turbidites. The nonlaminated sediments are the lateral seismic equivalent of Miocene and Pliocene hemipelagic clays cored on the flank of Nashville Seamount (Site 382).

As Challenger departed Site 382, two sonobuoy runs were attempted to determine the velocity structure of sediments and basement near the site. Neither sonobuoy functioned correctly.

The magnetic profile between Site 382 and Site 383 (Figure 1) gives some indication of the amplitude of the "J Anomaly," which is locally greater than 1000 gammas. The ship's track between Sites 383 and 384, however, parallels the magnetic trends and gives no indication of the nature of the $J$ Anomaly. Similarly, the track between Sites 384 and 385 is subparallel to the magnetic trends, and specific anomalies cannot be identified.

The profile approaching Site 385 goes south across Manning Seamount before crossing and recrossing Vogel Seamount. The profiler record between Manning and Vogel shows a number of moderately well defined reflectors below the Sohm turbidites, including Horizon $A^{\mathrm{C}}$ chert (cored at Site 385 ) and possibly Horizon $\beta$. Vogel Seamount is flanked on the east and north by drifts of acoustically nonlaminated sediments. Site 385 drilling shows this is substantially Miocene hemipelagic clay, like that at Site 382. Both Manning and Vogel seamounts have very high amplitude magnetic anomalies, unlike the small anomaly at Nashville Seamount.

Challenger's departure from Site 385 recrossed the flank of Vogel Seamount and then crossed a remarkably well developed set of abyssal sediment waves on the northern Bermuda Rise. These waves probably are formed by deposition of suspended sediment from the base of the westward flowing Gulf Stream Gyre (E. P. Laine, personal communication, 1976).

Near Bermuda, reflecting Horizon $A^{\mathrm{V}}$ is observed as a seaward extension of the acoustically opaque Bermuda Pedestal. The reflector was cored at Site 386 and consisted of upper Eocene to upper Oligocene volcaniclastic turbidites that record weathering of the Bermuda volcano.

While we were drilling at Site 386 , the satellite teletype suffered an irreparable mechanical failure, so that all subsequent navigation (including the position of Site 387) unfortunately is based on only celestial fixes, marginal quality Loran $\mathrm{C}$ fixes, and some radar fixes near Bermuda.

A single sonobuoy recording was attempted as Challenger crossed Site 386 enroute to Site 387, but the recording was unsuccessful.

The profile going westward from Site 386 to Site 387 shows that Horizon $A^{\mathrm{V}}$ gradually fades out away from Bermuda, and deeper reflectors become better defined. Horizon $\beta$ first appears in the seismic profile just east of Site 387 , but it is much easier to observe further west; at Site 387 , Horizon $\beta$ correlates with the top of Neocomian limestones underlying black clays. Horizon $A^{*}$ is a generally well-defined reflector near Site 387 , appearing in the upper part of an acoustically nonlaminated sequence of sediments overlying Horizon $\beta$. A post-site survey around site 387 shows highly reflective turbidites which flooded the western Bermuda Rise and covered Horizon $A^{*}$ during late Paleocene through middle Eocene time. Horizon $A^{\mathrm{C}}$ (chert within the turbidites) and Horizon $A^{\mathrm{T}}$ (top of the siliceous turbidites) cap this sequence.

When drilling was terminated at Site 387 on $6 \mathrm{Au}-$ gust 1975, the main swivel was found to be severely damaged, and because there were no spare parts on board, drilling had to be terminated for the duration of the leg. With 6 days remaining before the Challenger's scheduled port call in Norfolk, Virginia, we used part of the time for a detailed survey around Site 387 and for obtaining additional tie-lines on the southern Bermuda Rise between Sites 386 and 387.

During the survey around Site 387 , three sonobuoy recordings were attempted as the ship passed over the site. The first of these ( $1219 \mathrm{Z}, 7$ August) failed to record direct water-wave and sub-bottom information for a long enough period to be useful. The second sonobuoy (1800 Z, 7 August) failed to transmit, but a third sonobuoy $(0120 \mathrm{Z}, 8$ August) was successful. Analysis of this recording indicated an average velocity of $2.03 \pm 0.15 \mathrm{~km} / \mathrm{sec}$ for the sediment column, compared to $1.86 \mathrm{~km} / \mathrm{sec}$ derived from drillhole correlations. 
UNDERWAY GEOPHYSICS

The track proceeding across Site 387 to Norfolk at the end of the survey period (9-10 August) clearly recorded the older anomalies in the $M$ Series (Keathley sequence) and confirmed the position of Site 387 between anomalies $M-15$ and $M-16$. Acquisition of underway data was terminated above the top of the continental slope at $2200 \mathrm{hr}$ (GMT), 11 August 1975, in order to prepare for arrival in Norfolk, Virginia, on the morning of 12 August.

ACKNOWLEDGMENTS

I thank T. Gustafson and his shipboard technical staff for their excellent efforts in maintaining the geophysical gear and in routine data collection. T. Aitken of Lamont-Doherty Geological Observatory processed the underway data into the display format of Figure 1.

REFERENCES

Cain, J. C., Hendricks, S., Daniels, W. E., and Jensen, J. C., 1968. Computation of the main geomagnetic field from spherical harmonic expansions: Data User's Note NSSDC68-11, Greenbelt, Md.

Defense Mapping Agency Hydrographic Center, 1972. Bathymetric map of the Mediterranean Sea, Chart N.O. 310, scale 1:2,849,300, Washington, D.C.

Hsü, K. J., Cita, M. B. and Ryan, W. B. F., 1973. The origin of the Mediterranean evaporites In Ryan, W. B. F., Hsü, K. J., et al., Initial Reports of the Deep Sea Drilling Projest, Volume 13: Washington (U.S. Government Printing Office), p. 1203-1231.

Ryan, W. B. F., Hsü, K. J., et al., 1973. Initial Reports of the Deep Sea Drilling Project, Volume 13: Washington (U.S. Government Printing Office).

Talwani, M., 1969. A computer system for the reduction, storage, and display of underway data acquired at sea: Lamont-Doherty Geol. Obs. of Columbia Univ., Tech. Rept. 1, CU-1-69 N00014-67-A-0108-0004.

Uchupi, E., 1971. Bathymetric atlas of the Atlantic, Caribbean and Gulf of Mexico: Woods Hole Oceanog. Inst. Ref. No. 71-72 (unpublished manuscript).

891 


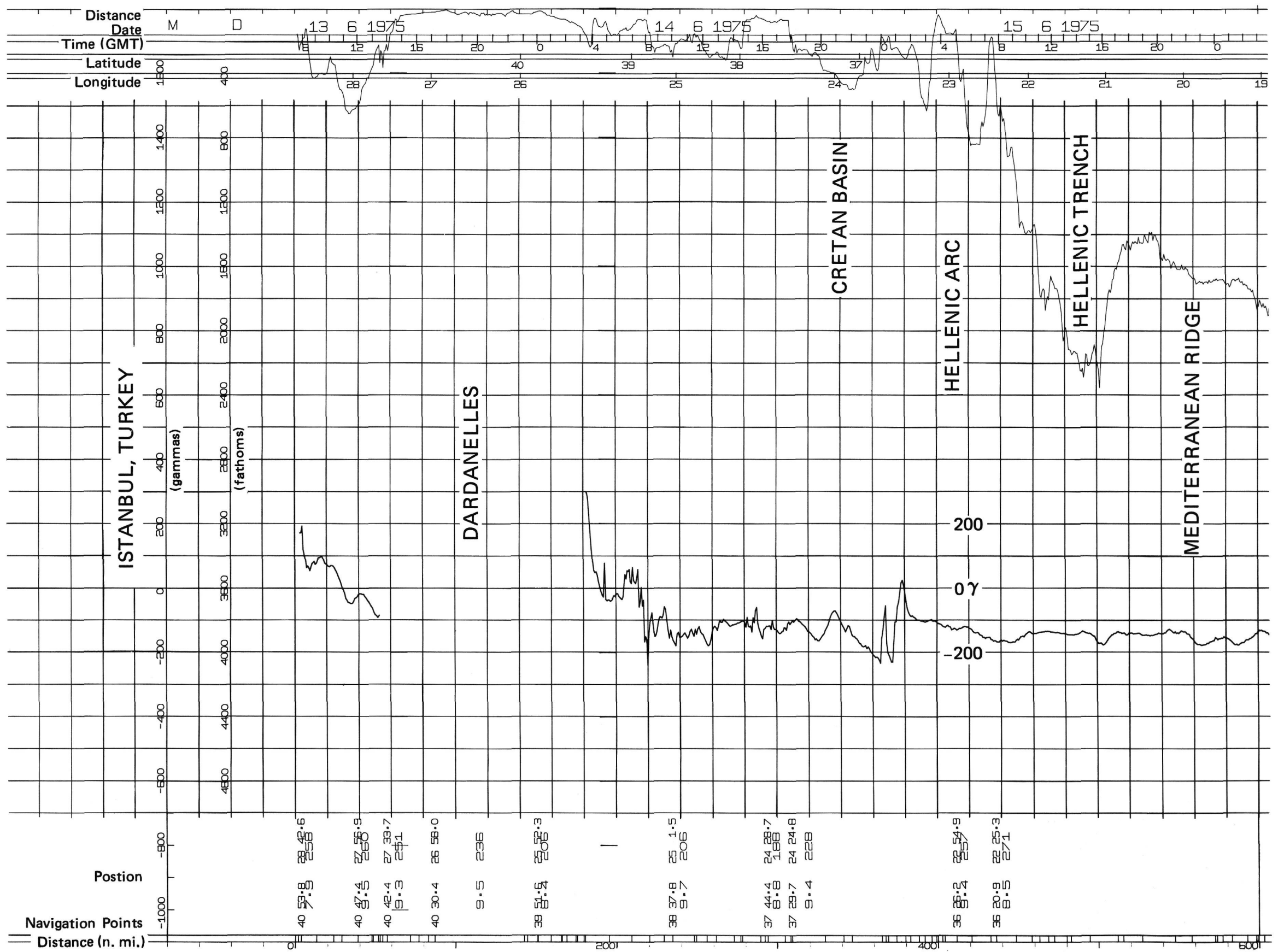




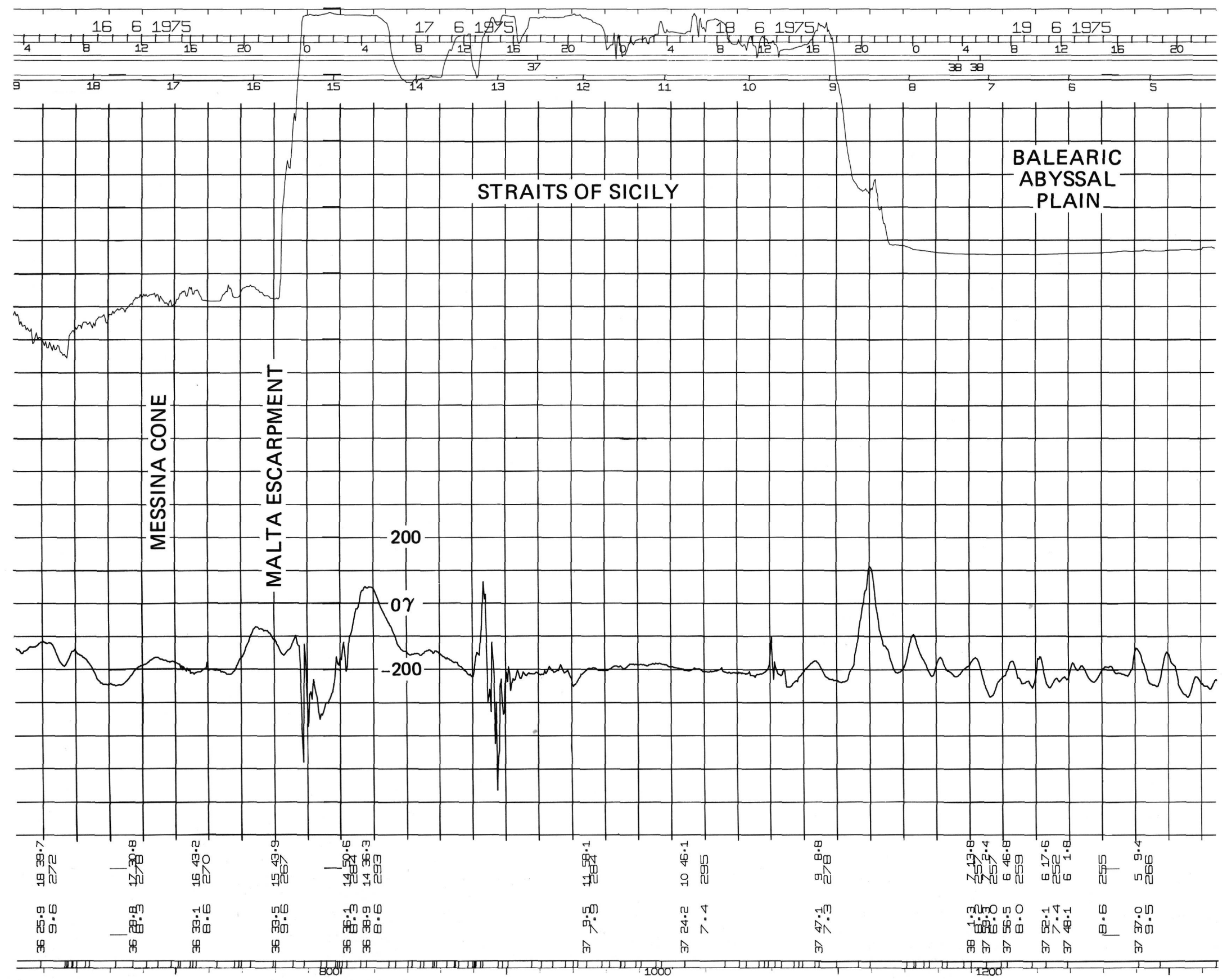

Figure 1. (Continued). 
Figure 1. (Continued). 


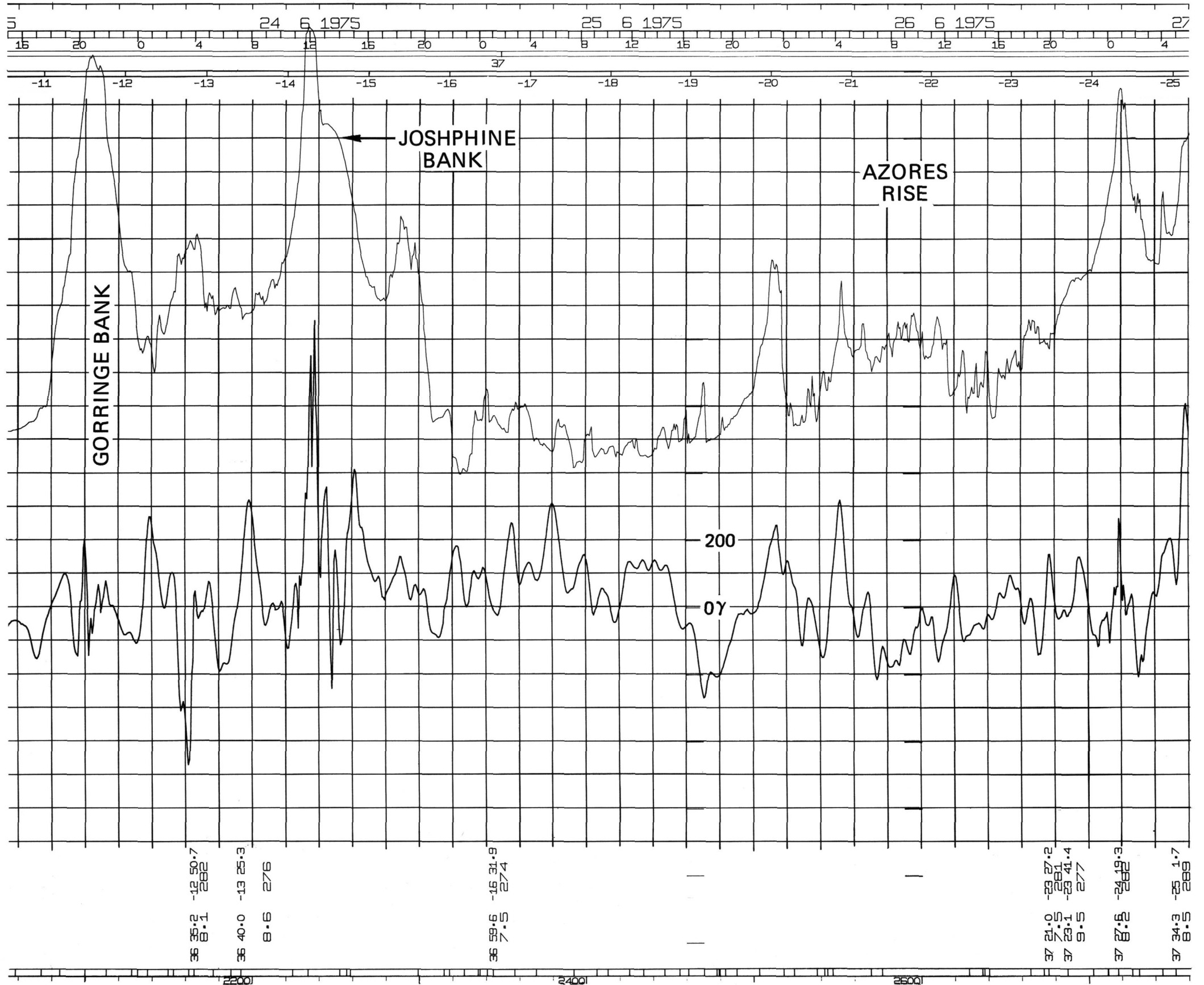




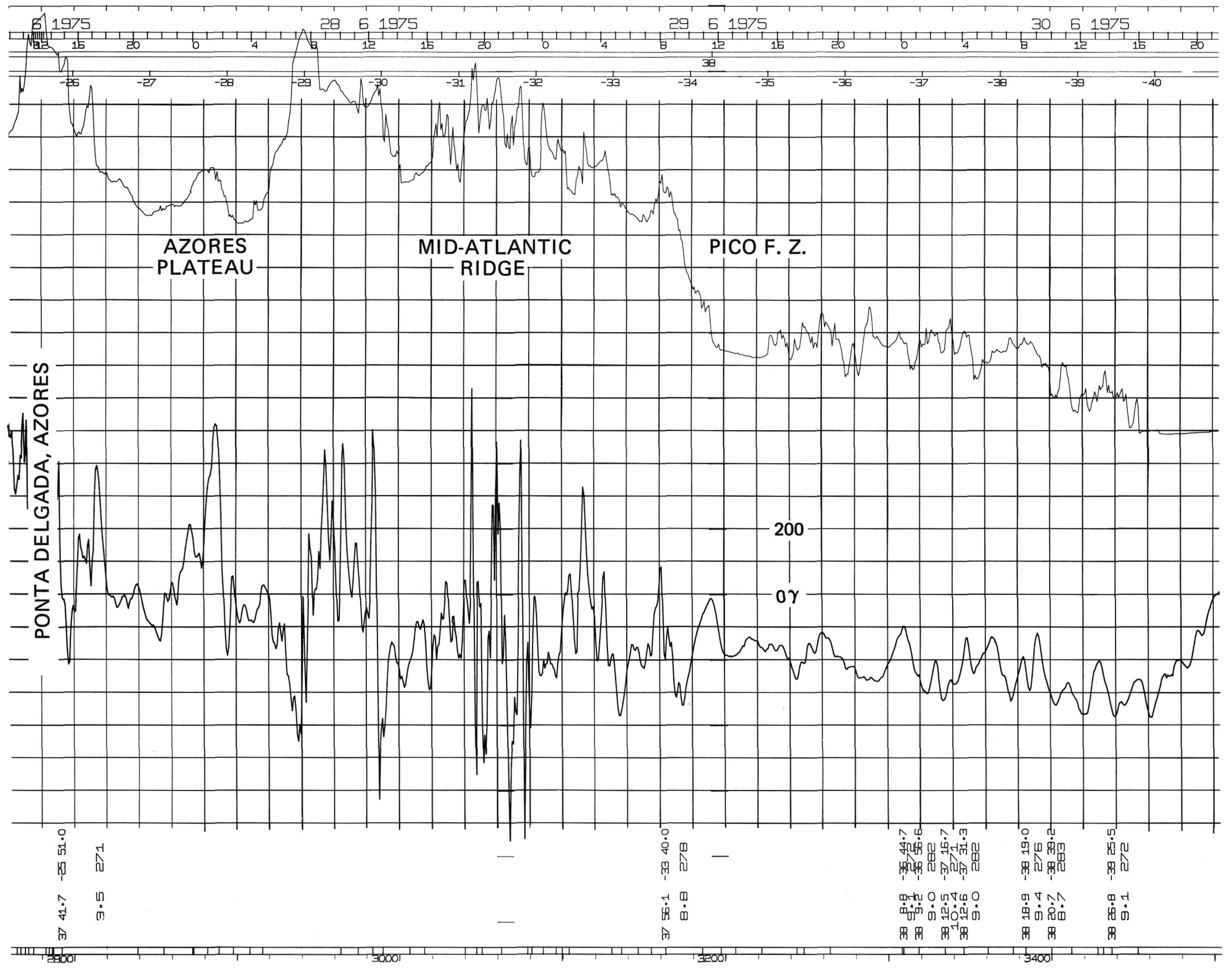

Figure 1. (Continued). 


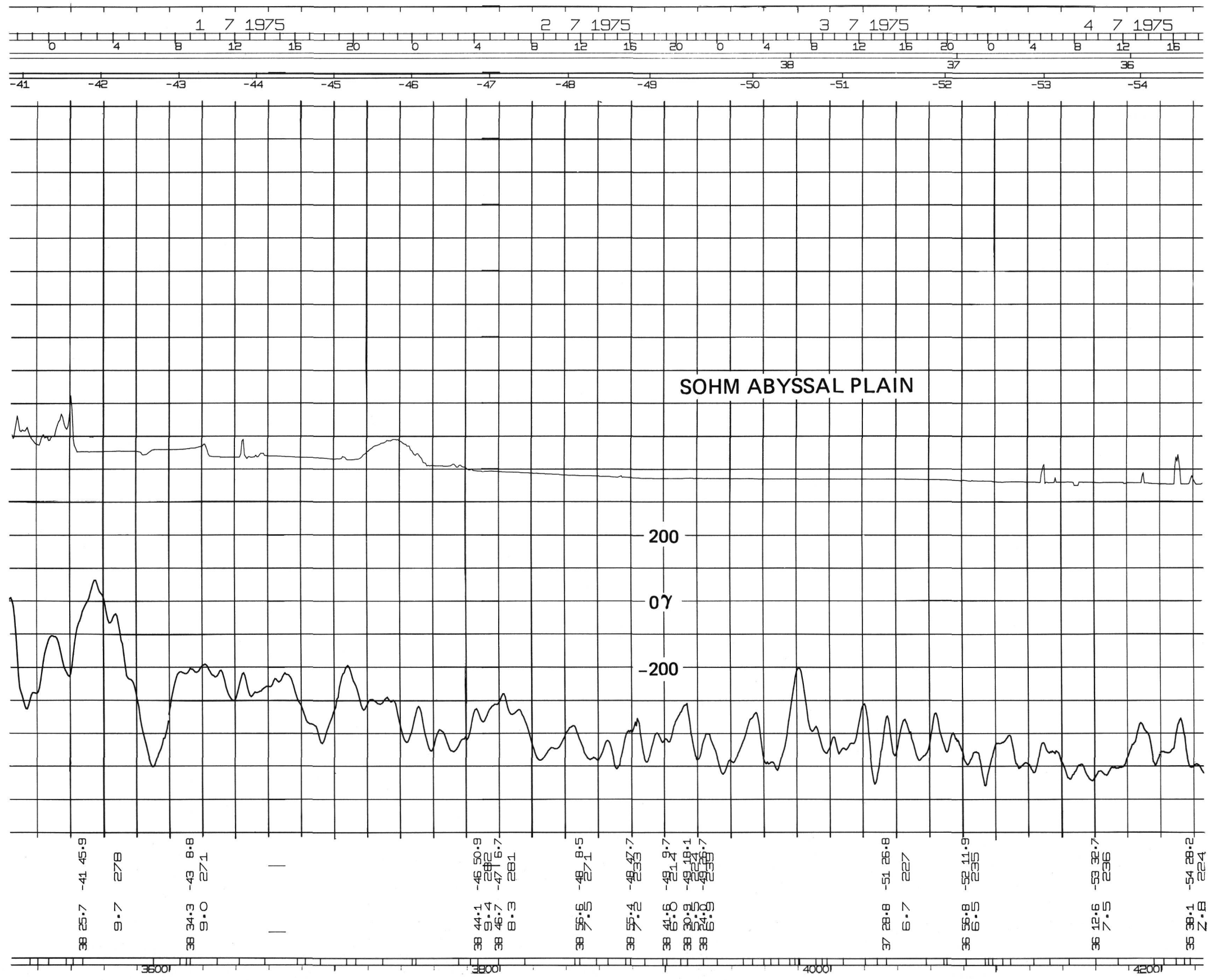




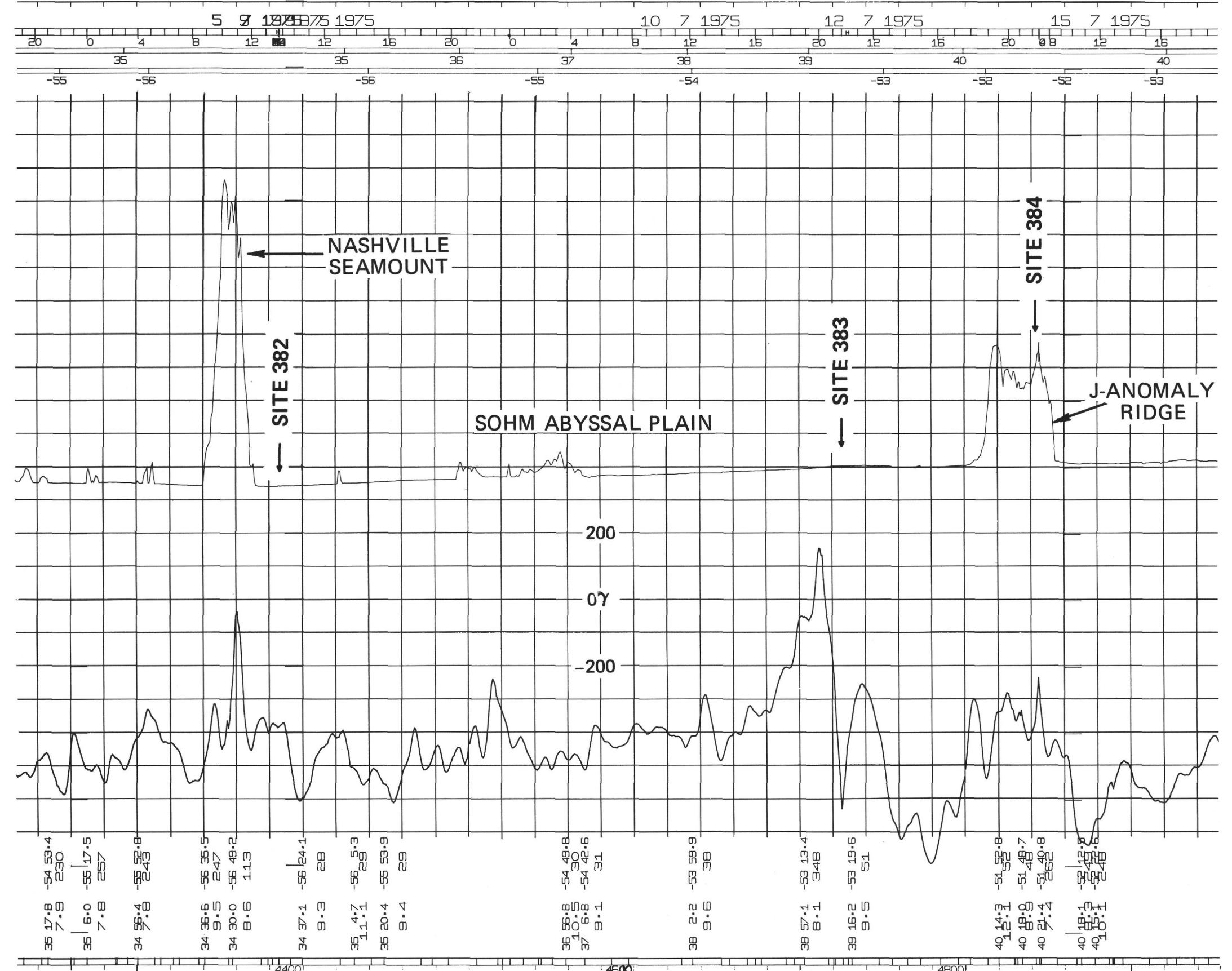

Figure 1. (Continued). 


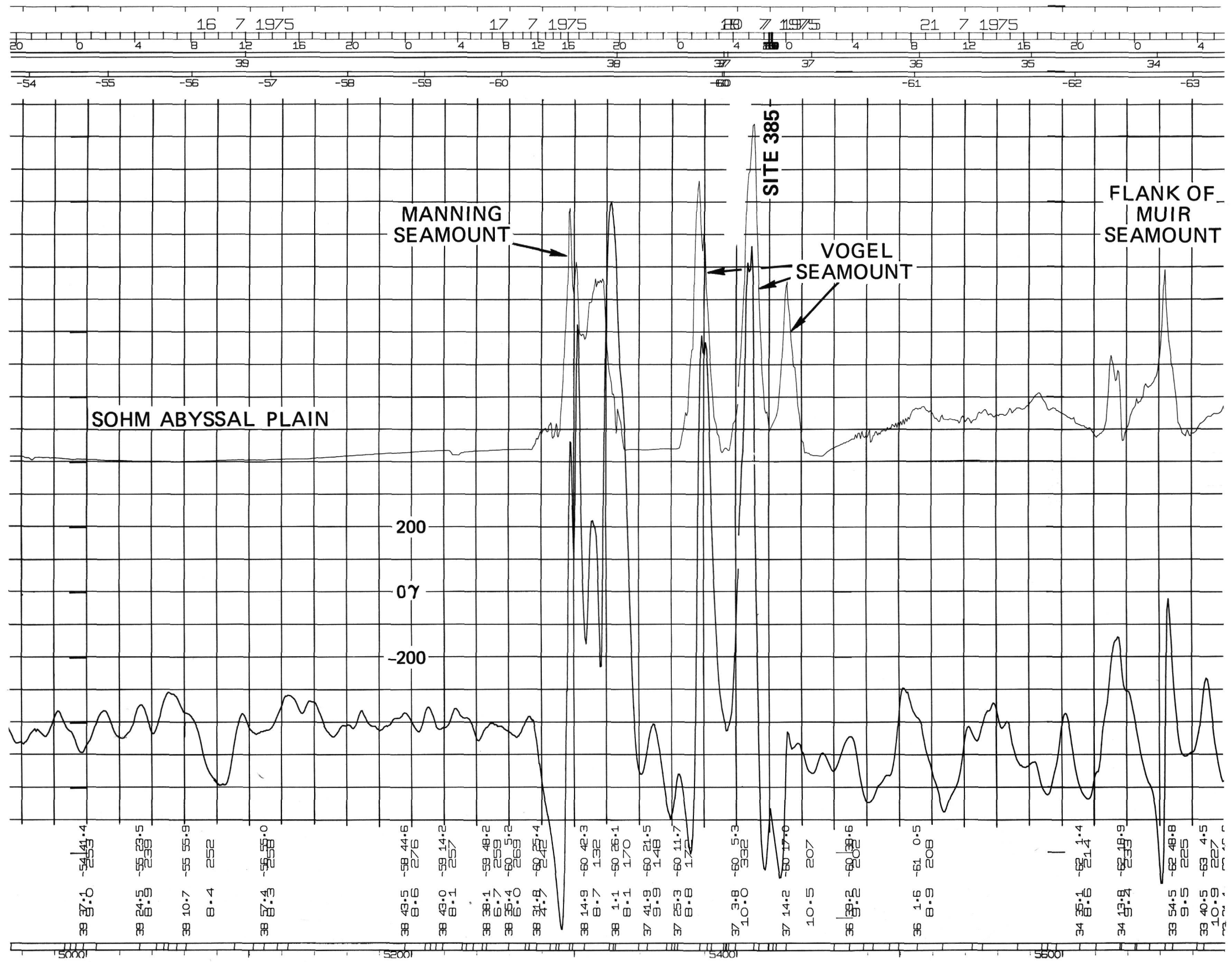

Figure 1. (Continued). 


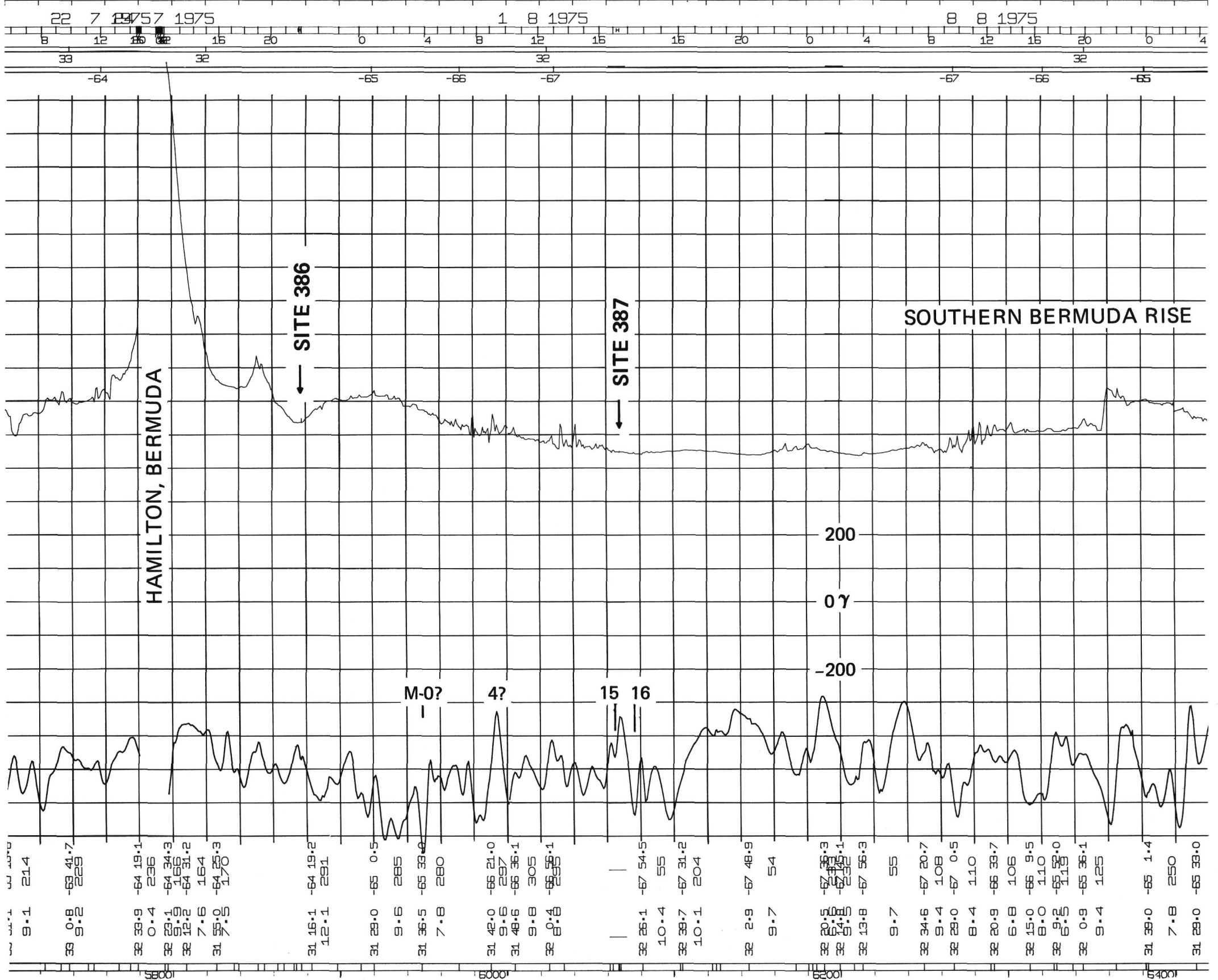

Figure 1. (Continued). 


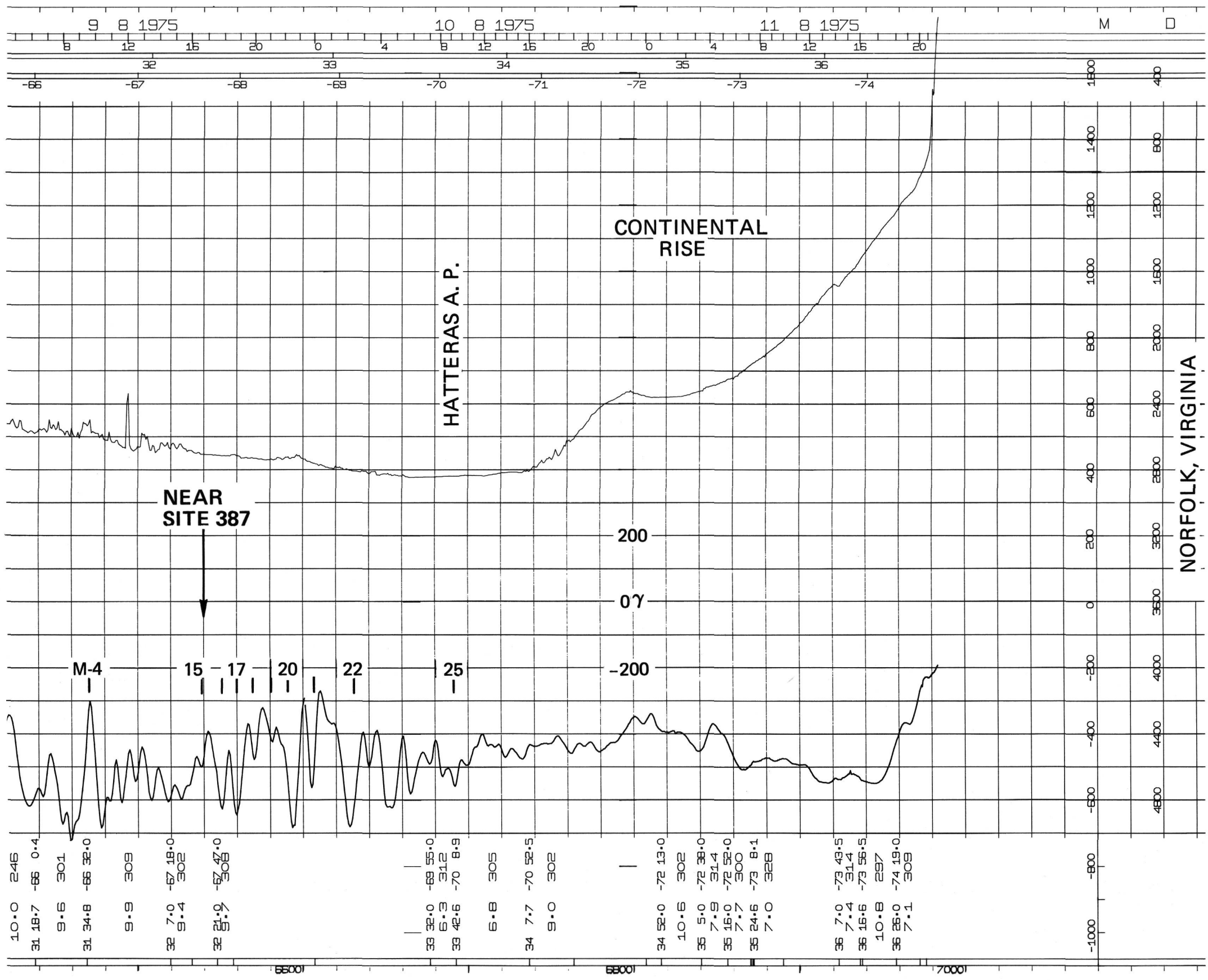

Figure 1. (Continued). 
TABLE 1

Underway Data for Glomar Challenger Leg 43: Navigation Points, Distance Along Track, Course and Speed Maintained Between Navigation Points, and Regional Magnetic Field Values

\begin{tabular}{|c|c|c|c|c|c|c|c|c|c|}
\hline Day & Mon & Year & $\operatorname{Time}^{\mathrm{a}}$ & Latitude & Longitude & Distance (n. mi.) & Speed (kt) & Course & Regional Mag \\
\hline 13 & 6 & 1975 & 716 & $40^{\circ} 54.8^{\prime}$ & $28^{\circ} 47.1^{\prime}$ & 0.0 & 7.7 & 254 & 46493 \\
\hline 13 & 6 & 1975 & 730 & $40^{\circ} 54.3^{\prime}$ & $28^{\circ} 44.8^{\prime}$ & 1.8 & 5.2 & 253 & 46486 \\
\hline 13 & 6 & 1975 & 750 & $40^{\circ} 53.8^{\prime}$ & $28^{\circ} 42.6^{\prime}$ & 3.5 & 7.9 & 258 & 46455 \\
\hline 13 & 6 & 1975 & 902 & $40^{\circ} 51.9^{\prime}$ & $28^{\circ} 30.3^{\prime}$ & 13.0 & 8.8 & 261 & 46421 \\
\hline 13. & 6 & 1975 & 1014 & $40^{\circ} 50.2^{\prime}$ & $28^{\circ} 16.5^{\prime}$ & 23.6 & 8.8 & 262 & 46406 \\
\hline 13 & 6 & 1975 & 1048 & $40^{\circ} 49.5^{\prime}$ & $28^{\circ} 10.0^{\prime}$ & 28.6 & 8.4 & 258 & 46372 \\
\hline 13 & 6 & 1975 & 1200 & $40^{\circ} 47.4^{\prime}$ & $27^{\circ} 56.9^{\prime}$ & 38.7 & 9.5 & 260 & 46345 \\
\hline 13 & 6 & 1975 & 1255 & $40^{\circ} 45.9^{\prime}$ & $27^{\circ} 45.6^{\prime}$ & 47.4 & 9.8 & 248 & 46342 \\
\hline 13 & 6 & 1975 & 1300 & $40^{\circ} 45.6^{\prime}$ & $27^{\circ} 44.6^{\prime}$ & 48.2 & 10.2 & 246 & 46328 \\
\hline 13 & 6 & 1975 & 1319 & $40^{\circ} 44.3^{\prime}$ & $27^{\circ} 40.7^{\prime}$ & 51.4 & 10.6 & 248 & 46324 \\
\hline 13 & 6 & 1975 & 1325 & $40^{\circ} 43.9^{\prime}$ & $27^{\circ} 39.4^{\prime}$ & 52.5 & 7.5 & 248 & 46317 \\
\hline 13 & 6 & 1975 & 1338 & $40^{\circ} 43.3^{\prime}$ & $27^{\circ} 37.4^{\prime}$ & 54.1 & 8.8 & 252 & 46306 \\
\hline 13 & 6 & 1975 & 1358 & $40^{\circ} 42.4^{\prime}$ & $27^{\circ} 33.7^{\prime}$ & 57.1 & 9.3 & 251 & 46250 \\
\hline 13 & 6 & 1975 & 1531 & $40^{\circ} 37.8^{\prime}$ & $27^{\circ} 15.6^{\prime}$ & 71.6 & 9.3 & 247 & 46228 \\
\hline 13 & 6 & 1975 & 1605 & $40^{\circ} 35.7^{\prime}$ & $27^{\circ} \quad 9.2^{\prime}$ & 76.9 & 9.3 & 236 & 46199 \\
\hline 13 & 6 & 1975 & 1643 & $40^{\circ} 32.4^{\prime}$ & $27^{\circ} \quad 2.8^{\prime}$ & 82.7 & 9.2 & 241 & 46181 \\
\hline 13 & 6 & 1975 & 1710 & $40^{\circ} 30.4^{\prime}$ & $26^{\circ} 58.0^{\prime}$ & 86.9 & 9.5 & 236 & 45910 \\
\hline 13 & 6 & 1975 & 2300 & $39^{\circ} 59.0^{\prime}$ & $25^{\circ} 58.1^{\prime}$ & 142.4 & 9.8 & 203 & 45898 \\
\hline 13 & 6 & 1975 & 2312 & $39^{\circ} 57.2^{\prime}$ & $25^{\circ} 57.1^{\prime}$ & 144.3 & 8.3 & 214 & 45861 \\
\hline 13 & 6 & 1975 & 2358 & $39^{\circ} 51.9^{\prime}$ & $25^{\circ} 52.5^{\prime}$ & 150.7 & 10.0 & 207 & 45859 \\
\hline 14 & 6 & 1975 & 000 & $39^{\circ} 51.6^{\prime}$ & $25^{\circ} 52.3^{\prime}$ & 151.0 & 8.4 & 206 & 45811 \\
\hline 14 & 6 & 1975 & 058 & $39^{\circ} 44.3^{\prime}$ & $25^{\circ} 47.7^{\prime}$ & 159.1 & 9.7 & 212 & 45773 \\
\hline 14 & 6 & 1975 & 138 & $39^{\circ} 38.8^{\prime}$ & $25^{\circ} 43.3^{\prime}$ & 165.6 & 9.3 & 210 & 45771 \\
\hline 14 & 6 & 1975 & 141 & $39^{\circ} 38.4^{\prime}$ & $25^{\circ} 43.0^{\prime}$ & 166.0 & 9.2 & 221 & 45754 \\
\hline 14 & 6 & 1975 & 200 & $39^{\circ} 36.2^{\prime}$ & $25^{\circ} 40.5^{\prime}$ & 169.0 & 8.9 & 206 & 45721 \\
\hline 14 & 6 & 1975 & 238 & $39^{\circ} 31.1^{\prime}$ & $25^{\circ} 37.3^{\prime}$ & 174.6 & 9.3 & 210 & 45715 \\
\hline 14 & 6 & 1975 & 244 & $39^{\circ} 30.3^{\prime}$ & $25^{\circ} 36.7^{\prime}$ & 175.6 & 8.9 & 213 & 45691 \\
\hline 14 & 6 & 1975 & 312 & $39^{\circ} 26.8^{\prime}$ & $25^{\circ} 33.8^{\prime}$ & 179.7 & 3.6 & 214 & 45686 \\
\hline 14 & 6 & 1975 & 326 & $39^{\circ} 26.1^{\prime}$ & $25^{\circ} 33.2^{\prime}$ & 180.6 & 8.9 & 207 & 45598 \\
\hline 14 & 6 & 1975 & 506 & $39^{\circ} 13.0^{\prime}$ & $25^{\circ} 24.4^{\prime}$ & 195.3 & 7.9 & 206 & 45514 \\
\hline 14 & 6 & 1975 & 652 & $39^{\circ} \quad 0.4^{\prime}$ & $25^{\circ} 16.5^{\prime}$ & 209.3 & 8.1 & 208 & 45448 \\
\hline 14 & 6 & 1975 & 814 & $38^{\circ} 50.6^{\prime}$ & $25^{\circ} \quad 9.8^{\prime}$ & 220.4 & 7.6 & 211 & 45394 \\
\hline 14 & 6 & 1975 & 925 & $38^{\circ} 42.9^{\prime}$ & $25^{\circ} \quad 3.8^{\prime}$ & 229.4 & 7.6 & 218 & 45393 \\
\hline 14 & 6 & 1975 & 926 & $38^{\circ} 42.8^{\prime}$ & $25^{\circ} \quad 3.7^{\prime}$ & 229.6 & 9.3 & 199 & 45361 \\
\hline 14 & 6 & 1975 & 1000 & $38^{\circ} 37.8^{\prime}$ & $25^{\circ} \quad 1.5^{\prime}$ & 234.9 & 9.7 & 206 & 45280 \\
\hline 14 & 6 & 1975 & 1123 & $38^{\circ} 25.7^{\prime}$ & $24^{\circ} 54.0^{\prime}$ & 248.3 & 9.3 & 210 & 45271 \\
\hline 14 & 6 & 1975 & 1132 & $38^{\circ} 24.5^{\prime}$ & $24^{\circ} 53.1^{\prime}$ & 249.7 & 9.8 & 203 & 45232 \\
\hline 14 & 6 & 1975 & 1212 & $38^{\circ} 18.5^{\prime}$ & $24^{\circ} 49.9^{\prime}$ & 256.2 & 9.6 & 203 & 45196 \\
\hline 14 & 6 & 1975 & 1248 & $38^{\circ} 13.2^{\prime}$ & $24^{\circ} 47.0^{\prime}$ & 262.0 & 9.5 & 211 & 45129 \\
\hline 14 & 6 & 1975 & 1358 & $38^{\circ} \quad 3.7^{\prime}$ & $24^{\circ} 39.7^{\prime}$ & 273.1 & 9.1 & 209 & 45104 \\
\hline 14 & 6 & 1975 & 1425 & $38^{\circ} \quad 0.1^{\prime}$ & $24^{\circ} 37.2^{\prime}$ & 277.2 & 9.2 & 202 & 45098 \\
\hline 14 & 6 & 1975 & 1432 & $37^{\circ} 59.1^{\prime}$ & $24^{\circ} 36.7^{\prime}$ & 278.2 & 9.0 & 204 & 45027 \\
\hline 14 & 6 & 1975 & 1548 & $37^{\circ} 48.7^{\prime}$ & $24^{\circ} 30.8^{\prime}$ & 289.6 & 9.1 & 204 & 45010 \\
\hline 14 & 6 & 1975 & 1606 & $37^{\circ} 46.2^{\prime}$ & $24^{\circ} 29.4^{\prime}$ & 292.4 & 9.4 & 197 & 44999 \\
\hline 14 & 6 & 1975 & 1618 & $37^{\circ} 44.4^{\prime}$ & $24^{\circ} 28.7^{\prime}$ & 294.3 & 8.8 & 188 & 44934 \\
\hline 14 & 6 & 1975 & 1730 & $37^{\circ} 33.9^{\prime}$ & $24^{\circ} 26.9^{\prime}$ & 304.9 & 8.5 & 197 & 44912 \\
\hline 14 & 6 & 1975 & 1755 & $37^{\circ} 30.5^{\prime}$ & $24^{\circ} 25.6^{\prime}$ & 308.4 & 8.8 & 218 & 44906 \\
\hline 14 & 6 & 1975 & 1802 & $37^{\circ} 29.7^{\prime}$ & $24^{\circ} 24.8^{\prime}$ & 309.4 & 9.4 & 228 & 44794 \\
\hline 14 & 6 & 1975 & 2012 & $37^{\circ} 16.0^{\prime}$ & $24^{\circ} \quad 5.8^{\prime}$ & 329.8 & 9.4 & 223 & 44728 \\
\hline 14 & 6 & 1975 & 2126 & $37^{\circ} \quad 7.6^{\prime}$ & $23^{\circ} 55.8^{\prime}$ & 341.4 & 9.8 & 223 & 44698 \\
\hline 14 & 6 & 1975 & 2158 & $37^{\circ} 03.8^{\prime}$ & $23^{\circ} 51.3^{\prime}$ & 346.6 & 9.2 & 224 & 44591 \\
\hline 15 & 6 & 1975 & 000 & $36^{\circ} 50.3^{\prime}$ & $23^{\circ} 35.1^{\prime}$ & 365.3 & 9.5 & 224 & 44579 \\
\hline 15 & 6 & 1975 & 014 & $36^{\circ} 48.7^{\prime}$ & $23^{\circ} 33.2^{\prime}$ & 367.5 & 8.9 & 228 & 44570 \\
\hline 15 & 6 & 1975 & 025 & $36^{\circ} 47.6^{\prime}$ & $23^{\circ} 31.7^{\prime}$ & 369.2 & 8.8 & 213 & 44538 \\
\hline 15 & 6 & 1975 & 100 & $36^{\circ} 43.3^{\prime}$ & $23^{\circ} 28.2^{\prime}$ & 374.3 & 9.3 & 211 & 44504 \\
\hline 15 & 6 & 1975 & 136 & $36^{\circ} 38.5^{\prime}$ & $23^{\circ} 24.6^{\prime}$ & 379.9 & 9.2 & 212 & 44485 \\
\hline 15 & 6 & 1975 & 156 & $36^{\circ} 35.9^{\prime}$ & $23^{\circ} 22.6^{\prime}$ & 382.9 & 9.4 & 210 & 44451 \\
\hline 15 & 6 & 1975 & 230 & $36^{\circ} 31.3^{\prime}$ & $23^{\circ} 19.3^{\prime}$ & 388.3 & 9.5 & 209 & 44428 \\
\hline 15 & 6 & 1975 & 253 & $36^{\circ} 28.1^{\prime}$ & $23^{\circ} 17.1^{\prime}$ & 391.9 & 9.5 & 221 & 44404 \\
\hline 15 & 6 & 1975 & 320 & $36^{\circ} 24.9^{\prime}$ & $23^{\circ} 13.6^{\prime}$ & 396.2 & 9.4 & 246 & 44391 \\
\hline
\end{tabular}


TABLE 1 - Continued

\begin{tabular}{|c|c|c|c|c|c|c|c|c|c|}
\hline Day & Mon & Year & $\operatorname{Time}^{\mathrm{a}}$ & Latitude & Longitude & Distance (n. mi.) & Speed $(k t)$ & Course & Regional Mag \\
\hline 15 & 6 & 1975 & 339 & $36^{\circ} 23.7^{\prime}$ & $23^{\circ} 10.2^{\prime}$ & 399.2 & 9.0 & 270 & 44389 \\
\hline 15 & 6 & 1975 & 346 & $36^{\circ} 23.7^{\prime}$ & $23^{\circ} 8.9^{\prime}$ & 400.2 & 9.4 & 272 & 44384 \\
\hline 15 & 6 & 1975 & 403 & $36^{\circ} 23.8^{\prime}$ & $23^{\circ} 5.6^{\prime}$ & 402.9 & 9.8 & 278 & 44382 \\
\hline 15 & 6 & 1975 & 412 & $36^{\circ} 24.0^{\prime}$ & $23^{\circ} 3.8^{\prime}$ & 404.3 & 9.6 & 287 & 44382 \\
\hline 15 & 6 & 1975 & 459 & $36^{\circ} 26.2^{\prime}$ & $22^{\circ} 54.9^{\prime}$ & 411.8 & 9.4 & 257 & 44351 \\
\hline 15 & 6 & 1975 & 558 & $36^{\circ} 24.1^{\prime}$ & $22^{\circ} 43.7^{\prime}$ & 421.1 & 8.5 & 253 & 44309 \\
\hline 15 & 6 & 1975 & 721 & $36^{\circ} 20.7^{\prime}$ & $22^{\circ} 29.7^{\prime}$ & 432.9 & 8.5 & 273 & 44303 \\
\hline 15 & 6 & 1975 & 746 & $36^{\circ} 20.9^{\prime}$ & $22^{\circ} 25.3^{\prime}$ & 436.4 & 8.5 & 271 & 44281 \\
\hline 15 & 6 & 1975 & 912 & $36^{\circ} 21.2^{\prime}$ & $22^{\circ} 10.1^{\prime}$ & 448.6 & 8.1 & 271 & 44264 \\
\hline 15 & 6 & 1975 & 1024 & $36^{\circ} 21.4^{\prime}$ & $21^{\circ} 58.0^{\prime}$ & 458.4 & 7.9 & 271 & 44255 \\
\hline 15 & 6 & 1975 & 1100 & $36^{\circ} 21.5^{\prime}$ & $21^{\circ} 52.1^{\prime}$ & 463.1 & 7.9 & 271 & 44245 \\
\hline 15 & 6 & 1975 & 1142 & $36^{\circ} 21.6^{\prime}$ & $21^{\circ} 45.2^{\prime}$ & 468.7 & 7.9 & 273 & 44239 \\
\hline 15 & 6 & 1975 & 1210 & $36^{\circ} 21.8^{\prime}$ & $21^{\circ} 40.6^{\prime}$ & 472.4 & 7.7 & 273 & 44227 \\
\hline 15 & 6 & 1975 & 1310 & $36^{\circ} 22.2^{\prime}$ & $21^{\circ} 31.0^{\prime}$ & 480.2 & 8.0 & 274 & 44223 \\
\hline 15 & 6 & 1975 & 1330 & $36^{\circ} 22.4^{\prime}$ & $21^{\circ} 27.7^{\prime}$ & 482.8 & 8.2 & 274 & 44207 \\
\hline 15 & 6 & 1975 & 1456 & $36^{\circ} 23.3^{\prime}$ & $21^{\circ} 13.2^{\prime}$ & 494.5 & 7.9 & 273 & 44179 \\
\hline 15 & 6 & 1975 & 1708 & $36^{\circ} 24.1^{\prime}$ & $20^{\circ} 51.5^{\prime}$ & 512.0 & 8.7 & 272 & 44173 \\
\hline 15 & 6 & 1975 & 1732 & $36^{\circ} 24.2^{\prime}$ & $20^{\circ} 47.2^{\prime}$ & 515.5 & 8.5 & 270 & 44152 \\
\hline 15 & 6 & 1975 & 1854 & $36^{\circ} 24.3^{\prime}$ & $20^{\circ} 32.7^{\prime}$ & 527.1 & 9.1 & 270 & 44114 \\
\hline 15 & 6 & 1975 & 2110 & $36^{\circ} 24.4^{\prime}$ & $20^{\circ} 7.2^{\prime}$ & 547.7 & 8.8 & 270 & 44095 \\
\hline 15 & 6 & 1975 & 2220 & $36^{\circ} 24.4^{\prime}$ & $19^{\circ} 54.4^{\prime}$ & 558.0 & 8.7 & 271 & 44071 \\
\hline 16 & 6 & 1975 & 000 & $36^{\circ} 24.7^{\prime}$ & $19^{\circ} 36.3^{\prime}$ & 572.5 & 9.1 & 270 & 44069 \\
\hline 16 & 6 & 1975 & 008 & $36^{\circ} 24.7^{\prime}$ & $19^{\circ} 34.8^{\prime}$ & 573.7 & 9.2 & 273 & 44064 \\
\hline 16 & 6 & 1975 & 030 & $36^{\circ} 24.9^{\prime}$ & $19^{\circ} 30.6^{\prime}$ & 577.1 & 8.8 & 271 & 44054 \\
\hline 16 & 6 & 1975 & 110 & $36^{\circ} 25.0^{\prime}$ & $19^{\circ} 23.3^{\prime}$ & 583.0 & 8.7 & 271 & 44037 \\
\hline 16 & 6 & 1975 & 216 & $36^{\circ} 25.1^{\prime}$ & $19^{\circ} 11.4^{\prime}$ & 592.6 & 8.8 & 272 & 44028 \\
\hline 16 & 6 & 1975 & 254 & $36^{\circ} 25.3^{\prime}$ & $19^{\circ} 4.5^{\prime}$ & 598.1 & 8.8 & 270 & 44027 \\
\hline 16 & 6 & 1975 & 300 & $36^{\circ} 25.3^{\prime}$ & $19^{\circ} 3.4^{\prime}$ & 599.0 & 9.0 & 273 & 44020 \\
\hline 16 & 6 & 1975 & 328 & $36^{\circ} 25.5^{\prime}$ & $18^{\circ} 58.2^{\prime}$ & 603.2 & 9.3 & 272 & 43996 \\
\hline 16 & 6 & 1975 & 504 & $36^{\circ} 25.9^{\prime}$ & $18^{\circ} 39.7^{\prime}$ & 618.1 & 9.6 & 272 & 43973 \\
\hline 16 & 6 & 1975 & 636 & $36^{\circ} 26.3^{\prime}$ & $18^{\circ} 21.4^{\prime}$ & 632.8 & 9.3 & 286 & 43973 \\
\hline 16 & 6 & 1975 & 643 & $36^{\circ} 26.6^{\prime}$ & $18^{\circ} 20.1^{\prime}$ & 633.9 & 9.6 & 270 & 43971 \\
\hline 16 & 6 & 1975 & 648 & $36^{\circ} 26.6^{\prime}$ & $18^{\circ} 19.1^{\prime}$ & 634.7 & 9.4 & 275 & 43968 \\
\hline 16 & 6 & 1975 & 703 & $36^{\circ} 26.8^{\prime}$ & $18^{\circ} 16.2^{\prime}$ & 637.1 & 10.5 & 247 & 43966 \\
\hline 16 & 6 & 1975 & 706 & $36^{\circ} 26.6^{\prime}$ & $18^{\circ} 15.6^{\prime}$ & 637.6 & 7.1 & 253 & 43958 \\
\hline 16 & 6 & 1975 & 726 & $36^{\circ} 25.9^{\prime}$ & $18^{\circ} 12.8^{\prime}$ & 639.9 & 7.3 & 275 & 43952 \\
\hline 16 & 6 & 1975 & 809 & $36^{\circ} 26.4^{\prime}$ & $18^{\circ} 6.3^{\prime}$ & 645.2 & 9.4 & 275 & 43949 \\
\hline 16 & 6 & 1975 & 824 & $36^{\circ} 26.6^{\prime}$ & $18^{\circ} 3.4^{\prime}$ & 647.5 & 9.0 & 274 & 43929 \\
\hline 16 & 6 & 1975 & $\begin{array}{r}024 \\
1010\end{array}$ & $36^{\circ} 27.8^{\prime}$ & $17^{\circ} 43.7^{\prime}$ & 663.4 & 8.9 & 276 & 43917 \\
\hline 16 & 6 & 1975 & 1120 & $36^{\circ} 28.8^{\prime}$ & $17^{\circ} 30.8^{\prime}$ & 673.9 & 8.3 & 278 & 43910 \\
\hline 16 & 6 & 1975 & 1220 & $36^{\circ} 29.9^{\prime}$ & $17^{\circ} 20.6^{\prime}$ & 682.1 & 8.7 & 278 & 43904 \\
\hline 16 & 6 & 1975 & 1308 & $36^{\circ} 30.9^{\prime}$ & $17^{\circ} 12.0^{\prime}$ & 689.1 & 8.3 & 279 & 43898 \\
\hline 16 & 6 & 1975 & 1406 & $36^{\circ} 32.1^{\prime}$ & $17^{\circ} 2.1^{\prime}$ & 697.2 & 8.5 & 274 & 43892 \\
\hline 16 & 6 & 1975 & 1436 & $36^{\circ} 32.4^{\prime}$ & $16^{\circ} 56.8^{\prime}$ & 701.4 & 7.7 & 274 & 43891 \\
\hline 16 & 6 & 1975 & 1446 & $36^{\circ} 32.5^{\prime}$ & $16^{\circ} 55.2^{\prime}$ & 702.7 & 5.2 & 278 & 43886 \\
\hline 16 & 6 & 1975 & 1556 & $36^{\circ} 33.4^{\prime}$ & $16^{\circ} 47.7^{\prime}$ & 708.8 & 5.1 & 266 & 43883 \\
\hline 16 & 6 & 1975 & 1615 & $36^{\circ} 33.3^{\prime}$ & $16^{\circ} 45.7^{\prime}$ & 710.4 & 8.1 & 264 & 43878 \\
\hline 16 & 6 & 1975 & 1630 & $36^{\circ} 33.1^{\prime}$ & $16^{\circ} 43.2^{\prime}$ & 712.4 & 8.6 & 270 & 43857 \\
\hline 16 & 6 & 1975 & 1758 & $36^{\circ} 33.1^{\prime}$ & $16^{\circ} 27.5^{\prime}$ & 725.0 & 8.1 & 268 & 43837 \\
\hline 16 & 6 & 1975 & 1916 & $36^{\circ} 32.7^{\prime}$ & $16^{\circ} 14.4^{\prime}$ & 735.6 & 8.2 & 286 & 43837 \\
\hline 16 & 6 & 1975 & 1935 & $36^{\circ} 33.4^{\prime}$ & $16^{\circ} 11.3^{\prime}$ & 738.2 & 7.9 & 270 & 43834 \\
\hline 16 & 6 & 1975 & 1946 & $36^{\circ} 33.4^{\prime}$ & $16^{\circ} 9.5^{\prime}$ & 739.6 & 8.6 & 269 & 43825 \\
\hline 16 & 6 & 1975 & 2024 & $36^{\circ} 33.3^{\prime}$ & $16^{\circ} 2.7^{\prime}$ & 745.1 & 8.5 & 271 & 43803 \\
\hline 16 & 6 & 1975 & 2200 & $36^{\circ} 33.5^{\prime}$ & $15^{\circ} 45.7^{\prime}$ & 758.7 & 8.7 & 270 & 43801 \\
\hline 16 & 6 & 1975 & 2210 & $36^{\circ} 33.5^{\prime}$ & $15^{\circ} 43.9^{\prime}$ & 760.2 & 9.6 & 267 & 43780 \\
\hline 16 & 6 & 1975 & 2316 & $36^{\circ} 33.0^{\prime}$ & $15^{\circ} 30.7^{\prime}$ & 770.8 & 9.2 & 270 & 43769 \\
\hline 17 & 6 & 1975 & 000 & $36^{\circ} 33.0^{\prime}$ & $15^{\circ} 22.3^{\prime}$ & 777.5 & 9.3 & 271 & 43753 \\
\hline 17 & 6 & 1975 & 106 & $36^{\circ} 33.1^{\prime}$ & $15^{\circ} 9.6^{\prime}$ & 787.7 & 8.6 & 268 & 43747 \\
\hline 17 & 6 & 1975 & 130 & $36^{\circ} 33.0^{\prime}$ & $15^{\circ} 5.3^{\prime}$ & 791.2 & 8.6 & 284 & 43747 \\
\hline 17 & 6 & 1975 & 208 & $36^{\circ} 34.3^{\prime}$ & $14^{\circ} 58.7^{\prime}$ & 796.7 & 9.1 & 281 & 43746 \\
\hline
\end{tabular}


TABLE 1 - Continued

\begin{tabular}{|c|c|c|c|c|c|c|c|c|c|}
\hline Day & Mon & Year & $\operatorname{Time}^{\mathrm{a}}$ & Latitude & Longitude & Distance (n. mi.) & Speed (kt) & Course & Regional Mag \\
\hline 17 & 6 & 1975 & 215 & $36^{\circ} 34.5^{\prime}$ & $14^{\circ} 57.4^{\prime}$ & 797.7 & 9.2 & 294 & 43747 \\
\hline 17 & 6 & 1975 & 223 & $36^{\circ} 35.0^{\prime}$ & $14^{\circ} 56.0^{\prime}$ & 799.0 & 9.0 & 284 & 43747 \\
\hline 17 & 6 & 1975 & 234 & $36^{\circ} 35.4^{\prime}$ & $14^{\circ} 54.0^{\prime}$ & 800.6 & 8.5 & 284 & 43747 \\
\hline 17 & 6 & 1975 & 254 & $36^{\circ} 36.1^{\prime}$ & $14^{\circ} 50.6^{\prime}$ & 803.4 & 8.3 & 284 & 43747 \\
\hline 17 & 6 & 1975 & 354 & $36^{\circ} 38.1^{\prime}$ & $14^{\circ} 40.5^{\prime}$ & 811.8 & 8.0 & 283 & 43746 \\
\hline 17 & 6 & 1975 & 420 & $36^{\circ} 38.9^{\prime}$ & $14^{\circ} 36.3^{\prime}$ & 815.2 & 8.6 & 293 & 43760 \\
\hline 17 & 6 & 1975 & 554 & $36^{\circ} 44.2^{\prime}$ & $14^{\circ} 20.9^{\prime}$ & 828.7 & 7.8 & 277 & 43757 \\
\hline 17 & 6 & 1975 & 625 & $36^{\circ} 44.7^{\prime}$ & $14^{\circ} 15.9^{\prime}$ & 832.7 & 8.0 & 267 & 43742 \\
\hline 17 & 6 & 1975 & 721 & $36^{\circ} 44.3^{\prime}$ & $14^{\circ} 6.6^{\prime}$ & 840.2 & 7.6 & 284 & 43742 \\
\hline 17 & 6 & 1975 & 740 & $36^{\circ} 44.9^{\prime}$ & $14^{\circ} 3.7^{\prime}$ & 842.6 & 4.8 & 270 & 43742 \\
\hline 17 & 6 & 1975 & 741 & $36^{\circ} 44.9^{\prime}$ & $14^{\circ} 3.6^{\prime}$ & 842.7 & 7.5 & 272 & 43735 \\
\hline 17 & 6 & 1975 & 822 & $36^{\circ} 45.1^{\prime}$ & $13^{\circ} 57.2^{\prime}$ & 847.8 & 7.4 & 291 & 43737 \\
\hline 17 & 6 & 1975 & 838 & $36^{\circ} 45.8^{\prime}$ & $13^{\circ} 54.9^{\prime}$ & 849.8 & 7.4 & 272 & 43730 \\
\hline 17 & 6 & 1975 & 922 & $36^{\circ} 46.0^{\prime}$ & $13^{\circ} 48.1^{\prime}$ & 855.2 & 7.6 & 264 & 43728 \\
\hline 17 & 6 & 1975 & 929 & $36^{\circ} 45.9^{\prime}$ & $13^{\circ} 47.0^{\prime}$ & 856.1 & 6.9 & 300 & 43730 \\
\hline 17 & 6 & 1975 & 941 & $36^{\circ} 46.6^{\prime}$ & $13^{\circ} 45.5^{\prime}$ & 857.5 & 7.4 & 266 & 43718 \\
\hline 17 & 6 & 1975 & 1032 & $36^{\circ} 46.2^{\prime}$ & $13^{\circ} 37.7^{\prime}$ & 863.7 & 7.2 & 269 & 43710 \\
\hline 17 & 6 & 1975 & 1110 & $36^{\circ} 46.1^{\prime}$ & $13^{\circ} 32.0^{\prime}$ & 868.3 & 7.7 & 270 & 43709 \\
\hline 17 & 6 & 1975 & 1115 & $36^{\circ} 46.1^{\prime}$ & $13^{\circ} 31.2^{\prime}$ & 869.0 & 7.7 & 246 & 43701 \\
\hline 17 & 6 & 1975 & 1130 & $36^{\circ} 45.3^{\prime}$ & $13^{\circ} 29.0^{\prime}$ & 870.9 & 7.1 & 279 & 43698 \\
\hline 17 & 6 & 1975 & 1218 & $36^{\circ} 46.2^{\prime}$ & $13^{\circ} 22.0^{\prime}$ & 876.6 & 7.2 & 276 & 43696 \\
\hline 17 & 6 & 1975 & 1242 & $36^{\circ} 46.5^{\prime}$ & $13^{\circ} 18.4^{\prime}$ & 879.5 & 6.7 & 321 & 43699 \\
\hline 17 & 6 & 1975 & 1250 & $36^{\circ} 47.2^{\prime}$ & $13^{\circ} 17.7^{\prime}$ & 880.4 & 7.2 & 270 & 43698 \\
\hline 17 & 6 & 1975 & 1254 & $36^{\circ} 47.2^{\prime}$ & $13^{\circ} 17.1^{\prime}$ & 880.8 & 4.8 & 270 & 43698 \\
\hline 17 & 6 & 1975 & 1258 & $36^{\circ} 47.2^{\prime}$ & $13^{\circ} 16.7^{\prime}$ & 881.2 & 4.8 & 270 & 43698 \\
\hline 17 & 6 & 1975 & 1300 & $36^{\circ} 47.2^{\prime}$ & $13^{\circ} 16.5^{\prime}$ & 881.3 & 8.5 & 278 & 43696 \\
\hline 17 & 6 & 1975 & 1320 & $36^{\circ} 47.6^{\prime}$ & $13^{\circ} 13.0^{\prime}$ & 884.2 & 7.7 & 274 & 43694 \\
\hline 17 & 6 & 1975 & 1330 & $36^{\circ} 47.7^{\prime}$ & $13^{\circ} 11.4^{\prime}$ & 885.4 & 7.6 & 288 & 43699 \\
\hline 17 & 6 & 1975 & 1444 & $36^{\circ} 50.6^{\prime}$ & $13^{\circ} 0.3^{\prime}$ & 894.8 & 7.1 & 288 & 43700 \\
\hline 17 & 6 & 1975 & 1506 & $36^{\circ} 51.4^{\prime}$ & $12^{\circ} 57.2^{\prime}$ & 897.4 & 7.5 & 290 & 43703 \\
\hline 17 & 6 & 1975 & 1532 & $36^{\circ} 52.5^{\prime}$ & $12^{\circ} 53.4^{\prime}$ & 900.6 & 8.1 & 294 & 43719 \\
\hline 17 & 6 & 1975 & 1718 & $36^{\circ} 58.3^{\prime}$ & $12^{\circ} 37.0^{\prime}$ & 915.0 & 8.3 & 295 & 43727 \\
\hline 17 & 6 & 1975 & 1800 & $37^{\circ} 00.8^{\prime}$ & $12^{\circ} 30.4^{\prime}$ & 920.8 & 8.2 & 290 & 43732 \\
\hline 17 & 6 & 1975 & 1850 & $37^{\circ} 03.1^{\prime}$ & $12^{\circ} 22.3^{\prime}$ & 927.7 & 8.3 & 292 & 43739 \\
\hline 17 & 6 & 1975 & 1945 & $37^{\circ} 05.9^{\prime}$ & $12^{\circ} 13.4^{\prime}$ & 935.3 & 8.4 & 288 & 43741 \\
\hline 17 & 6 & 1975 & 2018 & $37^{\circ} 07.3^{\prime}$ & $12^{\circ} 07.9^{\prime}$ & 939.9 & 8.2 & 285 & 43742 \\
\hline 17 & 6 & 1975 & 2038 & $37^{\circ} 08.0^{\prime}$ & $12^{\circ} 04.6^{\prime}$ & 942.6 & 7.7 & 286 & 43743 \\
\hline 17 & 6 & 1975 & 2120 & $37^{\circ} 09.5^{\prime}$ & $11^{\circ} 58.1^{\prime}$ & 948.0 & 7.9 & 284 & 43745 \\
\hline 17 & 6 & 1975 & 2230 & $37^{\circ} 11.8^{\prime}$ & $11^{\circ} 46.9^{\prime}$ & 957.2 & 7.7 & 288 & 43747 \\
\hline 17 & 6 & 1975 & 2306 & $37^{\circ} 13.2^{\prime}$ & $11^{\circ} 41.4^{\prime}$ & 961.8 & 7.4 & 285 & 43748 \\
\hline 18 & 6 & 1975 & 000 & $37^{\circ} 14.9^{\prime}$ & $11^{\circ} 33.3^{\prime}$ & 968.5 & 7.8 & 293 & 43749 \\
\hline 18 & 6 & 1975 & 002 & $37^{\circ} 15.0^{\prime}$ & $11^{\circ} 33.0^{\prime}$ & 968.8 & 7.2 & 287 & 43750 \\
\hline 18 & 6 & 1975 & 022 & $37^{\circ} 15.7^{\prime}$ & $11^{\circ} 30.1^{\prime}$ & 971.2 & 6.8 & 282 & 43749 \\
\hline 18 & 6 & 1975 & 120 & $37^{\circ} 17.1^{\prime}$ & $11^{\circ} 22.0^{\prime}$ & 977.8 & 7.1 & 279 & 43748 \\
\hline 18 & 6 & 1975 & 146 & $37^{\circ} 17.6^{\prime}$ & $11^{\circ} 18.2^{\prime}$ & 980.8 & 6.9 & 285 & 43750 \\
\hline 18 & 6 & 1975 & 304 & $37^{\circ} 19.9^{\prime}$ & $11^{\circ} 07.3^{\prime}$ & 989.8 & 8.2 & 284 & 43750 \\
\hline 18 & 6 & 1975 & 328 & $37^{\circ} 20.7^{\prime}$ & $11^{\circ} 03.3^{\prime}$ & 993.1 & 7.9 & 284 & 43752 \\
\hline 18 & 6 & 1975 & 515 & $37^{\circ} 24.1^{\prime}$ & $10^{\circ} 46.2^{\prime}$ & 1007.1 & 7.7 & 321 & 43753 \\
\hline 18 & 6 & 1975 & 516 & $37^{\circ} 24.2^{\prime}$ & $10^{\circ} 46.1^{\prime}$ & 1007.2 & 7.4 & 295 & 43786 \\
\hline 18 & 6 & 1975 & 834 & $37^{\circ} 34.4^{\prime}$ & $10^{\circ} 18.2^{\prime}$ & 1031.6 & 7.2 & 298 & 43801 \\
\hline 18 & 6 & 1975 & 946 & $37^{\circ} 38.4^{\prime}$ & $10^{\circ} 08.5^{\prime}$ & 1040.3 & 7.3 & 276 & 43797 \\
\hline 18 & 6 & 1975 & 1020 & $37^{\circ} 38.8^{\prime}$ & $10^{\circ} 03.3^{\prime}$ & 1044.4 & 6.9 & 277 & 43793 \\
\hline 18 & 6 & 1975 & 1128 & $37^{\circ} 39.8^{\prime}$ & $9^{\circ} 53.5^{\prime}$ & 1052.2 & 7.5 & 277 & 43791 \\
\hline 18 & 6 & 1975 & 1154 & $37^{\circ} 40.2^{\prime}$ & $9^{\circ} 49.4^{\prime}$ & 1055.5 & 7.7 & 275 & 43789 \\
\hline 18 & 6 & 1975 & 1212 & $37^{\circ} 40.4^{\prime}$ & $9^{\circ} 46.5^{\prime}$ & 1057.8 & 7.5 & 281 & 43789 \\
\hline 18 & 6 & 1975 & 1245 & $37^{\circ} 41.2^{\prime}$ & $9^{\circ} 41.4^{\prime}$ & 1061.9 & 7.5 & 286 & 43791 \\
\hline 18 & 6 & 1975 & 1316 & $37^{\circ} 42.3^{\prime}$ & $9^{\circ} 36.7^{\prime}$ & 1065.8 & 7.3 & 286 & 43794 \\
\hline 18 & 6 & 1975 & 1418 & $37^{\circ} 44.4^{\prime}$ & $9^{\circ} 27.5^{\prime}$ & 1073.4 & 7.7 & 282 & 43794 \\
\hline 18 & 6 & 1975 & 1440 & $37^{\circ} 45.0^{\prime}$ & $9^{\circ} 24.0^{\prime}$ & 1076.2 & 6.8 & 283 & 43794 \\
\hline 18 & 6 & 1975 & 1500 & $37^{\circ} 45.5^{\prime}$ & $9^{\circ} 21.2^{\prime}$ & 1078.5 & 6.9 & 279 & 43792 \\
\hline
\end{tabular}


TABLE 1 - Continued

\begin{tabular}{|c|c|c|c|c|c|c|c|c|c|}
\hline Day & Mon & Year & Time $^{\mathrm{a}}$ & Latitude & Longitude & Distance (n. mi.) & Speed $(k t)$ & Course & Regional Mag \\
\hline $\begin{array}{l}18 \\
18 \\
18 \\
18 \\
18\end{array}$ & $\begin{array}{l}6 \\
6 \\
6 \\
6 \\
6\end{array}$ & $\begin{array}{l}1975 \\
1975 \\
1975 \\
1975 \\
1975\end{array}$ & $\begin{array}{l}1604 \\
1626 \\
1754 \\
1940 \\
2034\end{array}$ & $\begin{array}{l}37^{\circ} 46.7^{\prime} \\
37^{\circ} 47.1^{\prime} \\
37^{\circ} 48.5^{\prime} \\
37^{\circ} 50.2^{\prime} \\
37^{\circ} 51.3^{\prime}\end{array}$ & $\begin{array}{l}9^{\circ} 12.0^{\prime} \\
9^{\circ} 08.8^{\prime} \\
8^{\circ} 55.4^{\prime} \\
8^{\circ} 38.9^{\prime} \\
8^{\circ} 30.1^{\prime}\end{array}$ & $\begin{array}{l}1085.8 \\
1088.4 \\
1099.1 \\
1112.2 \\
1119.2\end{array}$ & $\begin{array}{l}7.0 \\
7.3 \\
7.4 \\
7.8 \\
7.7\end{array}$ & $\begin{array}{l}279 \\
278 \\
277 \\
279 \\
280\end{array}$ & $\begin{array}{l}43791 \\
43786 \\
43779 \\
43777 \\
43775\end{array}$ \\
\hline $\begin{array}{l}18 \\
19 \\
19 \\
19 \\
19\end{array}$ & $\begin{array}{l}6 \\
6 \\
6 \\
6 \\
6\end{array}$ & $\begin{array}{l}1975 \\
1975 \\
1975 \\
1975 \\
1975\end{array}$ & $\begin{array}{r}2326 \\
000 \\
008 \\
048 \\
114\end{array}$ & $\begin{array}{l}37^{\circ} 55.3^{\prime} \\
37^{\circ} 56.1^{\prime} \\
37^{\circ} 56.3^{\prime} \\
37^{\circ} 57.0^{\prime} \\
37^{\circ} 57.3^{\prime}\end{array}$ & $\begin{array}{l}8^{\circ} 02.5^{\prime} \\
7^{\circ} 57.2^{\prime} \\
7^{\circ} 55.9^{\prime} \\
7^{\circ} 49.6^{\prime} \\
7^{\circ} 45.5^{\prime}\end{array}$ & $\begin{array}{l}1141.4 \\
1145.6 \\
1146.7 \\
1151.7 \\
1155.0\end{array}$ & $\begin{array}{l}7.5 \\
7.8 \\
7.5 \\
7.5 \\
7.5 \\
\end{array}$ & $\begin{array}{l}281 \\
281 \\
278 \\
275 \\
279\end{array}$ & $\begin{array}{l}43774 \\
43774 \\
43773 \\
43770 \\
43768\end{array}$ \\
\hline $\begin{array}{l}19 \\
19 \\
19 \\
19 \\
19\end{array}$ & $\begin{array}{l}6 \\
6 \\
6 \\
6 \\
6\end{array}$ & $\begin{array}{l}1975 \\
1975 \\
1975 \\
1975 \\
1975\end{array}$ & $\begin{array}{l}216 \\
240 \\
402 \\
435 \\
542\end{array}$ & $\begin{array}{l}37^{\circ} 58.5^{\prime} \\
37^{\circ} 58.9^{\prime} \\
38^{\circ} 00.4^{\prime} \\
38^{\circ} 01.3^{\prime} \\
37^{\circ} 59.3^{\prime}\end{array}$ & $\begin{array}{l}7^{\circ} 35.8^{\prime} \\
7^{\circ} 32.1^{\prime} \\
7^{\circ} 19.4^{\prime} \\
7^{\circ} 13.8^{\prime} \\
7^{\circ} 02.4^{\prime}\end{array}$ & $\begin{array}{l}1162.7 \\
1165.6 \\
1175.8 \\
1180.3 \\
1189.5\end{array}$ & $\begin{array}{l}7.4 \\
7.4 \\
8.2 \\
8.2 \\
6.0\end{array}$ & $\begin{array}{l}278 \\
279 \\
282 \\
257 \\
257\end{array}$ & $\begin{array}{l}43767 \\
43764 \\
43765 \\
43741 \\
43717\end{array}$ \\
\hline $\begin{array}{l}19 \\
19 \\
19 \\
19 \\
19\end{array}$ & $\begin{array}{l}6 \\
6 \\
6 \\
6 \\
6\end{array}$ & $\begin{array}{l}1975 \\
1975 \\
1975 \\
1975 \\
1975\end{array}$ & $\begin{array}{r}712 \\
738 \\
932 \\
1040 \\
1226\end{array}$ & $\begin{array}{l}37^{\circ} 57.3^{\prime} \\
37^{\circ} 56.5^{\prime} \\
37^{\circ} 53.5^{\prime} \\
37^{\circ} 52.1^{\prime} \\
37^{\circ} 48.1^{\prime}\end{array}$ & $\begin{array}{l}6^{\circ} 51.2^{\prime} \\
6^{\circ} 46.8^{\prime} \\
6^{\circ} 28.0^{\prime} \\
6^{\circ} 17.6^{\prime} \\
6^{\circ} 01.8^{\prime}\end{array}$ & $\begin{array}{l}1198.5 \\
1202.1 \\
1217.2 \\
1225.5 \\
1238.6\end{array}$ & $\begin{array}{l}8.2 \\
8.0 \\
7.3 \\
7.4 \\
8.6\end{array}$ & $\begin{array}{l}257 \\
259 \\
260 \\
252 \\
255\end{array}$ & $\begin{array}{l}43708 \\
43671 \\
43652 \\
43611 \\
43490\end{array}$ \\
\hline $\begin{array}{l}19 \\
19 \\
19 \\
19 \\
20\end{array}$ & $\begin{array}{l}6 \\
6 \\
6 \\
6 \\
6\end{array}$ & $\begin{array}{l}1975 \\
1975 \\
1975 \\
1975 \\
1975\end{array}$ & $\begin{array}{r}1724 \\
1846 \\
2240 \\
2316 \\
000\end{array}$ & $\begin{array}{l}37^{\circ} 37.0^{\prime} \\
37^{\circ} 36.1^{\prime} \\
37^{\circ} 30.6^{\prime} \\
37^{\circ} 30.0^{\prime} \\
37^{\circ} 29.1^{\prime}\end{array}$ & $\begin{array}{l}5^{\circ} 09.4^{\prime} \\
4^{\circ} 53.1^{\prime} \\
4^{\circ} 10.7^{\prime} \\
4^{\circ} 04.0^{\prime} \\
3^{\circ} 55.8^{\prime}\end{array}$ & $\begin{array}{l}1281.6 \\
1294.5 \\
1328.6 \\
1333.9 \\
1340.5\end{array}$ & $\begin{array}{l}9.5 \\
8.7 \\
8.9 \\
9.0 \\
9.0\end{array}$ & $\begin{array}{l}266 \\
261 \\
264 \\
262 \\
262\end{array}$ & $\begin{array}{l}43470 \\
43396 \\
43386 \\
43373 \\
43366\end{array}$ \\
\hline $\begin{array}{l}20 \\
20 \\
20 \\
20 \\
20\end{array}$ & $\begin{array}{l}6 \\
6 \\
6 \\
6 \\
6\end{array}$ & $\begin{array}{l}1975 \\
1975 \\
1975 \\
1975 \\
1975\end{array}$ & $\begin{array}{l}024 \\
118 \\
216 \\
304 \\
334\end{array}$ & $\begin{array}{l}37^{\circ} 28.6^{\prime} \\
37^{\circ} 27.4^{\prime} \\
37^{\circ} 26.2^{\prime} \\
37^{\circ} 25.3^{\prime} \\
37^{\circ} 24.9^{\prime}\end{array}$ & $\begin{array}{l}3^{\circ} 51.3^{\prime} \\
3^{\circ} 41.1^{\prime} \\
3^{\circ} 30.7^{\prime} \\
3^{\circ} 21.5^{\prime} \\
3^{\circ} 16.1^{\prime}\end{array}$ & $\begin{array}{l}1344.1 \\
1352.3 \\
1360.6 \\
1368.0 \\
1372.3\end{array}$ & $\begin{array}{l}9.1 \\
8.6 \\
9.2 \\
8.6 \\
8.6\end{array}$ & $\begin{array}{l}262 \\
262 \\
263 \\
265 \\
264\end{array}$ & $\begin{array}{l}43350 \\
43333 \\
43319 \\
43312 \\
43287\end{array}$ \\
\hline $\begin{array}{l}20 \\
20 \\
20 \\
20 \\
20\end{array}$ & $\begin{array}{l}6 \\
6 \\
6 \\
6 \\
6\end{array}$ & $\begin{array}{l}1975 \\
1975 \\
1975 \\
1975 \\
1975\end{array}$ & $\begin{array}{l}518 \\
540 \\
642 \\
828 \\
848\end{array}$ & $\begin{array}{l}37^{\circ} 23.4^{\prime} \\
37^{\circ} 23.1^{\prime} \\
37^{\circ} 21.6^{\prime} \\
37^{\circ} 19.0^{\prime} \\
37^{\circ} 18.5^{\prime}\end{array}$ & $\begin{array}{l}2^{\circ} 57.4^{\prime} \\
2^{\circ} 53.3^{\prime} \\
2^{\circ} 42.0^{\prime} \\
2^{\circ} 23.0^{\prime} \\
2^{\circ} 19.5^{\prime}\end{array}$ & $\begin{array}{l}1387.2 \\
1390.5 \\
1399.6 \\
1414.9 \\
1417.7\end{array}$ & $\begin{array}{l}8.9 \\
8.8 \\
8.7 \\
8.5 \\
8.6\end{array}$ & $\begin{array}{l}265 \\
261 \\
260 \\
260 \\
259\end{array}$ & $\begin{array}{l}43281 \\
43262 \\
43230 \\
43223 \\
43182\end{array}$ \\
\hline $\begin{array}{l}20 \\
20 \\
20 \\
20 \\
20\end{array}$ & $\begin{array}{l}6 \\
6 \\
6 \\
6 \\
6\end{array}$ & $\begin{array}{l}1975 \\
1975 \\
1975 \\
1975 \\
1975\end{array}$ & $\begin{array}{l}1100 \\
1136 \\
1218 \\
1308 \\
1328\end{array}$ & $\begin{array}{l}37^{\circ} 15.0^{\prime} \\
37^{\circ} 13.7^{\prime} \\
37^{\circ} 12.1^{\prime} \\
37^{\circ} 10.4^{\prime} \\
37^{\circ} 09.7^{\prime}\end{array}$ & $\begin{array}{l}1^{\circ} 56.2^{\prime} \\
1^{\circ} 49.9^{\prime} \\
1^{\circ} 42.6^{\prime} \\
1^{\circ} 33.2^{\prime} \\
1^{\circ} 29.6^{\prime}\end{array}$ & $\begin{array}{l}1436.6 \\
1441.8 \\
1447.8 \\
1455.5 \\
1458.5\end{array}$ & $\begin{array}{l}8.6 \\
8.6 \\
9.2 \\
8.9 \\
8.9\end{array}$ & $\begin{array}{l}255 \\
255 \\
257 \\
256 \\
258\end{array}$ & $\begin{array}{l}43168 \\
43152 \\
43133 \\
43125 \\
43105\end{array}$ \\
\hline $\begin{array}{l}20 \\
20 \\
20 \\
20 \\
20\end{array}$ & $\begin{array}{l}6 \\
6 \\
6 \\
6 \\
6\end{array}$ & $\begin{array}{l}1975 \\
1975 \\
1975 \\
1975 \\
1975\end{array}$ & $\begin{array}{l}1426 \\
1446 \\
1616 \\
1818 \\
1836\end{array}$ & $\begin{array}{l}37^{\circ} 07.9^{\prime} \\
37^{\circ} 07.2^{\prime} \\
37^{\circ} 04.2^{\prime} \\
37^{\circ} 00.1^{\prime} \\
36^{\circ} 57.6^{\prime}\end{array}$ & $\begin{array}{l}1^{\circ} 19.0^{\prime} \\
1^{\circ} 15.2^{\prime} \\
0^{\circ} 58.8^{\prime} \\
0^{\circ} 35.6^{\prime} \\
0^{\circ} 20.8^{\prime}\end{array}$ & $\begin{array}{l}1467.1 \\
1470.2 \\
1483.6 \\
1502.6 \\
1514.7\end{array}$ & $\begin{array}{l}9.3 \\
8.9 \\
9.3 \\
9.3 \\
8.7\end{array}$ & $\begin{array}{l}257 \\
257 \\
258 \\
258 \\
258\end{array}$ & $\begin{array}{l}43098 \\
43065 \\
43021 \\
42993 \\
42980\end{array}$ \\
\hline $\begin{array}{l}20 \\
20 \\
20 \\
20 \\
20\end{array}$ & $\begin{array}{l}6 \\
6 \\
6 \\
6 \\
6\end{array}$ & $\begin{array}{l}1975 \\
1975 \\
1975 \\
1975 \\
1975\end{array}$ & $\begin{array}{l}2015 \\
2044 \\
2124 \\
2156 \\
2315\end{array}$ & $\begin{array}{l}36^{\circ} 56.4^{\prime} \\
36^{\circ} 55.7^{\prime} \\
36^{\circ} 54.4^{\prime} \\
36^{\circ} 53.4^{\prime} \\
36^{\circ} 51.8^{\prime}\end{array}$ & $\begin{array}{l}0^{\circ} 13.9^{\prime} \\
0^{\circ} 08.7^{\prime} \\
0^{\circ} 01.7^{\prime} \\
0^{\circ}-3.9^{\prime} \\
0^{\circ}-13.3^{\prime}\end{array}$ & $\begin{array}{l}1520.3 \\
1524.5 \\
1530.3 \\
1534.9 \\
1542.6\end{array}$ & $\begin{array}{l}8.7 \\
8.6 \\
8.6 \\
5.8 \\
8.6\end{array}$ & $\begin{array}{l}260 \\
257 \\
257 \\
258 \\
257\end{array}$ & $\begin{array}{l}42971 \\
42958 \\
42947 \\
42929 \\
42923\end{array}$ \\
\hline $\begin{array}{l}20 \\
21 \\
21 \\
21 \\
21\end{array}$ & $\begin{array}{l}6 \\
6 \\
6 \\
6 \\
6\end{array}$ & $\begin{array}{l}1975 \\
1975 \\
1975 \\
1975 \\
1975\end{array}$ & $\begin{array}{r}2334 \\
000 \\
012 \\
122 \\
226\end{array}$ & $\begin{array}{l}36^{\circ} 51.2^{\prime} \\
36^{\circ} 50.4^{\prime} \\
36^{\circ} 50.0^{\prime} \\
36^{\circ} 47.8^{\prime} \\
36^{\circ} 45.6^{\prime}\end{array}$ & $\begin{array}{l}0^{\circ}-16.6^{\prime} \\
0^{\circ}-21.3^{\prime} \\
0^{\circ}-23.4^{\prime} \\
0^{\circ}-35.5^{\prime} \\
0^{\circ}-45.9^{\prime}\end{array}$ & $\begin{array}{l}1545.3 \\
1549.1 \\
1550.8 \\
1560.8 \\
1569.4\end{array}$ & $\begin{array}{l}8.9 \\
8.7 \\
8.5 \\
8.1 \\
8.4\end{array}$ & $\begin{array}{l}258 \\
257 \\
257 \\
255 \\
253\end{array}$ & $\begin{array}{l}42914 \\
42910 \\
42887 \\
42865 \\
42855\end{array}$ \\
\hline $\begin{array}{l}21 \\
21 \\
21 \\
21 \\
21\end{array}$ & $\begin{array}{l}6 \\
6 \\
6 \\
6 \\
6\end{array}$ & $\begin{array}{l}1975 \\
1975 \\
1975 \\
1975 \\
1975\end{array}$ & $\begin{array}{l}250 \\
344 \\
412 \\
432 \\
550\end{array}$ & $\begin{array}{l}36^{\circ} 44.6^{\prime} \\
36^{\circ} 42.6^{\prime} \\
36^{\circ} 41.7^{\prime} \\
36^{\circ} 41.0^{\prime} \\
36^{\circ} 38.9^{\prime}\end{array}$ & $\begin{array}{r}0^{\circ}-49.9^{\prime} \\
0^{\circ} 59.1^{\prime} \\
-1^{\circ} 03.9^{\prime} \\
-1^{\circ} 07.7^{\prime} \\
-1^{\circ} 21.0^{\prime}\end{array}$ & $\begin{array}{l}1572.7 \\
1580.4 \\
1584.3 \\
1587.5 \\
1598.3\end{array}$ & $\begin{array}{l}8.5 \\
8.5 \\
9.4 \\
8.4 \\
7.7\end{array}$ & $\begin{array}{l}255 \\
257 \\
257 \\
259 \\
261\end{array}$ & $\begin{array}{l}42836 \\
42826 \\
42819 \\
42796 \\
42790\end{array}$ \\
\hline
\end{tabular}


TABLE 1 - Continued

\begin{tabular}{|c|c|c|c|c|c|c|c|c|c|}
\hline Day & Mon & Year & Time $^{a}$ & Latitude & Longitude & Distance (n. mi.) & Speed (kt) & Course & Regional Mag \\
\hline $\begin{array}{l}21 \\
21 \\
21 \\
21 \\
21\end{array}$ & $\begin{array}{l}6 \\
6 \\
6 \\
6 \\
6\end{array}$ & $\begin{array}{l}1975 \\
1975 \\
1975 \\
1975 \\
1975\end{array}$ & $\begin{array}{r}616 \\
732 \\
942 \\
1025 \\
1048\end{array}$ & $\begin{array}{l}36^{\circ} 38.4^{\prime} \\
36^{\circ} 36.6^{\prime} \\
36^{\circ} 33.7^{\prime} \\
36^{\circ} 32.6^{\prime} \\
36^{\circ} 32.1^{\prime}\end{array}$ & $\begin{array}{l}-1^{\circ} 25.1^{\prime} \\
-1^{\circ} 37.6^{\prime} \\
-2^{\circ} 00.2^{\prime} \\
-2^{\circ} 07.4^{\prime} \\
-2^{\circ} 11.0^{\prime}\end{array}$ & $\begin{array}{l}1601.7 \\
1611.8 \\
1630.2 \\
1636.1 \\
1639.0\end{array}$ & $\begin{array}{l}8.0 \\
8.5 \\
8.2 \\
7.6 \\
7.3\end{array}$ & $\begin{array}{l}260 \\
261 \\
259 \\
260 \\
270\end{array}$ & $\begin{array}{l}42770 \\
42736 \\
42724 \\
42719 \\
42718\end{array}$ \\
\hline $\begin{array}{l}21 \\
21 \\
21 \\
21 \\
21\end{array}$ & $\begin{array}{l}6 \\
6 \\
6 \\
6 \\
6\end{array}$ & $\begin{array}{l}1975 \\
1975 \\
1975 \\
1975 \\
1975\end{array}$ & $\begin{array}{l}1050 \\
1130 \\
1526 \\
1712 \\
1732\end{array}$ & $\begin{array}{l}36^{\circ} 32.1^{\prime} \\
36^{\circ} 31.6^{\prime} \\
36^{\circ} 25.8^{\prime} \\
36^{\circ} 23.6^{\prime} \\
36^{\circ} 23.1^{\prime}\end{array}$ & $\begin{array}{l}-2^{\circ} 11.3^{\prime} \\
-2^{\circ} 18.8^{\prime} \\
-2^{\circ} 55.9^{\prime} \\
-3^{\circ} 12.9^{\prime} \\
-3^{\circ} 16.4^{\prime}\end{array}$ & $\begin{array}{l}1639.3 \\
1645.3 \\
1675.7 \\
1689.6 \\
1692.4\end{array}$ & $\begin{array}{l}9.1 \\
7.7 \\
7.8 \\
8.6 \\
8.9\end{array}$ & $\begin{array}{l}265 \\
259 \\
261 \\
260 \\
259\end{array}$ & $\begin{array}{l}42711 \\
42649 \\
42625 \\
42620 \\
42600\end{array}$ \\
\hline $\begin{array}{l}21 \\
21 \\
21 \\
21 \\
21\end{array}$ & $\begin{array}{l}6 \\
6 \\
6 \\
6 \\
6\end{array}$ & $\begin{array}{l}1975 \\
1975 \\
1975 \\
1975 \\
1975\end{array}$ & $\begin{array}{l}1842 \\
2030 \\
2250 \\
2300 \\
2316\end{array}$ & $\begin{array}{l}36^{\circ} 21.2^{\prime} \\
36^{\circ} 17.8^{\prime} \\
36^{\circ} 12.4^{\prime} \\
36^{\circ} 12.0^{\prime} \\
36^{\circ} 12.6^{\prime}\end{array}$ & $\begin{array}{l}-3^{\circ} 29.1^{\prime} \\
-3^{\circ} 46.6^{\prime} \\
-4^{\circ} 05.5^{\prime} \\
-4^{\circ} 06.7^{\prime} \\
-4^{\circ} 08.4^{\prime}\end{array}$ & $\begin{array}{l}1702.8 \\
1717.3 \\
1733.5 \\
1734.6 \\
1736.1\end{array}$ & $\begin{array}{l}8.1 \\
6.9 \\
6.3 \\
5.6 \\
6.4\end{array}$ & $\begin{array}{l}256 \\
250 \\
248 \\
294 \\
246\end{array}$ & $\begin{array}{l}42567 \\
42519 \\
42516 \\
42520 \\
42504\end{array}$ \\
\hline $\begin{array}{l}22 \\
22 \\
22 \\
22 \\
22\end{array}$ & $\begin{array}{l}6 \\
6 \\
6 \\
6 \\
6\end{array}$ & $\begin{array}{l}1975 \\
1975 \\
1975 \\
1975 \\
1975\end{array}$ & $\begin{array}{l}000 \\
052 \\
115 \\
150 \\
200\end{array}$ & $\begin{array}{l}36^{\circ} 10.7^{\prime} \\
36^{\circ} 08.5^{\prime} \\
36^{\circ} 08.0^{\prime} \\
36^{\circ} 07.5^{\prime} \\
36^{\circ} 07.0^{\prime}\end{array}$ & $\begin{array}{l}-4^{\circ} 13.7^{\prime} \\
-4^{\circ} 19.8^{\prime} \\
-4^{\circ} 22.5^{\prime} \\
-4^{\circ} 26.6^{\prime} \\
-4^{\circ} 27.7^{\prime}\end{array}$ & $\begin{array}{l}1740.7 \\
1746.1 \\
1748.4 \\
1751.7 \\
1752.7\end{array}$ & $\begin{array}{l}6.2 \\
5.8 \\
5.7 \\
6.1 \\
5.7\end{array}$ & $\begin{array}{l}246 \\
257 \\
261 \\
241 \\
261\end{array}$ & $\begin{array}{l}42485 \\
42480 \\
42475 \\
42471 \\
42468\end{array}$ \\
\hline $\begin{array}{l}22 \\
22 \\
22 \\
22 \\
22\end{array}$ & $\begin{array}{l}6 \\
6 \\
6 \\
6 \\
6\end{array}$ & $\begin{array}{l}1975 \\
1975 \\
1975 \\
1975 \\
1975\end{array}$ & $\begin{array}{l}220 \\
240 \\
245 \\
322 \\
415\end{array}$ & $\begin{array}{l}36^{\circ} 06.7^{\prime} \\
36^{\circ} 07.0^{\prime} \\
36^{\circ} 07.0^{\prime} \\
36^{\circ} 07.3^{\prime} \\
36^{\circ} 07.7^{\prime}\end{array}$ & $\begin{array}{l}-4^{\circ} 30.0^{\prime} \\
-4^{\circ} 33.3^{\prime} \\
-4^{\circ} 33.9^{\prime} \\
-4^{\circ} 38.4^{\prime} \\
-4^{\circ} 45.6^{\prime}\end{array}$ & $\begin{array}{l}1754.6 \\
1757.3 \\
1757.8 \\
1761.4 \\
1767.3\end{array}$ & $\begin{array}{l}8.1 \\
5.8 \\
5.9 \\
6.6 \\
4.0\end{array}$ & $\begin{array}{l}276 \\
270 \\
275 \\
274 \\
282\end{array}$ & $\begin{array}{l}42468 \\
42468 \\
42468 \\
42468 \\
42469\end{array}$ \\
\hline $\begin{array}{l}22 \\
22 \\
22 \\
22 \\
22\end{array}$ & $\begin{array}{l}6 \\
6 \\
6 \\
6 \\
6\end{array}$ & $\begin{array}{l}1975 \\
1975 \\
1975 \\
1975 \\
1975\end{array}$ & $\begin{array}{l}430 \\
510 \\
637 \\
638 \\
730\end{array}$ & $\begin{array}{l}36^{\circ} 07.9^{\prime} \\
36^{\circ} 08.2^{\prime} \\
36^{\circ} 08.8^{\prime} \\
36^{\circ} 08.8^{\prime} \\
36^{\circ} 06.7^{\prime}\end{array}$ & $\begin{array}{l}-4^{\circ} 46.8^{\prime} \\
-4^{\circ} 52.2^{\prime} \\
-5^{\circ} 06.9^{\prime} \\
-5^{\circ} 07.1^{\prime} \\
-5^{\circ} 15.6^{\prime}\end{array}$ & $\begin{array}{l}1768.3 \\
1772.6 \\
1784.5 \\
1784.7 \\
1791.9\end{array}$ & $\begin{array}{l}6.6 \\
8.2 \\
9.7 \\
8.3 \\
8.4\end{array}$ & $\begin{array}{l}274 \\
273 \\
270 \\
253 \\
248\end{array}$ & $\begin{array}{l}42469 \\
42468 \\
42468 \\
42450 \\
42439\end{array}$ \\
\hline $\begin{array}{l}22 \\
22 \\
22 \\
22 \\
22\end{array}$ & $\begin{array}{l}6 \\
6 \\
6 \\
6 \\
6\end{array}$ & $\begin{array}{l}1975 \\
1975 \\
1975 \\
1975 \\
1975\end{array}$ & $\begin{array}{r}755 \\
824 \\
1000 \\
1042 \\
1146\end{array}$ & $\begin{array}{l}36^{\circ} 05.4^{\prime} \\
36^{\circ} 02.5^{\prime} \\
35^{\circ} 56.2^{\prime} \\
35^{\circ} 57.8^{\prime} \\
35^{\circ} 57.7^{\prime}\end{array}$ & $\begin{array}{l}-5^{\circ} 19.6^{\prime} \\
-5^{\circ} 23.2^{\prime} \\
-5^{\circ} 35.7^{\prime} \\
-5^{\circ} 43.1^{\prime} \\
-5^{\circ} 54.4^{\prime}\end{array}$ & $\begin{array}{l}1795.3 \\
1799.4 \\
1811.4 \\
1817.6 \\
1826.7\end{array}$ & $\begin{array}{l}8.5 \\
7.4 \\
8.9 \\
8.6 \\
7.8\end{array}$ & $\begin{array}{l}225 \\
238 \\
285 \\
269 \\
272\end{array}$ & $\begin{array}{l}42416 \\
42366 \\
42375 \\
42370 \\
42370\end{array}$ \\
\hline $\begin{array}{l}22 \\
22 \\
22 \\
22 \\
22\end{array}$ & $\begin{array}{l}6 \\
6 \\
6 \\
6 \\
6\end{array}$ & $\begin{array}{l}1975 \\
1975 \\
1975 \\
1975 \\
1975\end{array}$ & $\begin{array}{l}1228 \\
1245 \\
1332 \\
1424 \\
1520\end{array}$ & $\begin{array}{l}35^{\circ} 57.9^{\prime} \\
35^{\circ} 57.9^{\prime} \\
35^{\circ} 58.7^{\prime} \\
35^{\circ} 59.3^{\prime} \\
35^{\circ} 59.3^{\prime}\end{array}$ & $\begin{array}{l}-6^{\circ} 01.1^{\prime} \\
-6^{\circ} 03.7^{\prime} \\
-6^{\circ} 11.0^{\prime} \\
-6^{\circ} 19.4^{\prime} \\
-6^{\circ} 28.5^{\prime}\end{array}$ & $\begin{array}{l}1832.1 \\
1834.2 \\
1840.2 \\
1847.0 \\
1854.4\end{array}$ & $\begin{array}{l}7.4 \\
7.6 \\
7.9 \\
7.9 \\
4.9\end{array}$ & $\begin{array}{l}270 \\
278 \\
275 \\
270 \\
270\end{array}$ & $\begin{array}{l}42369 \\
42373 \\
42375 \\
42372 \\
42372\end{array}$ \\
\hline $\begin{array}{l}22 \\
22 \\
22 \\
22 \\
22\end{array}$ & $\begin{array}{l}6 \\
6 \\
6 \\
6 \\
6\end{array}$ & $\begin{array}{l}1975 \\
1975 \\
1975 \\
1975 \\
1975\end{array}$ & $\begin{array}{l}1535 \\
1630 \\
1822 \\
2056 \\
2120\end{array}$ & $\begin{array}{l}35^{\circ} 59.3^{\prime} \\
35^{\circ} 59.3^{\prime} \\
36^{\circ} 00.8^{\prime} \\
36^{\circ} 03.1^{\prime} \\
36^{\circ} 03.6^{\prime}\end{array}$ & $\begin{array}{l}-6^{\circ} 30.0^{\prime} \\
-6^{\circ} 38.9^{\prime} \\
-6^{\circ} 58.0^{\prime} \\
-7^{\circ} 25.2^{\prime} \\
-7^{\circ} 29.3^{\prime}\end{array}$ & $\begin{array}{l}1855.6 \\
1862.8 \\
1878.3 \\
1900.4 \\
1903.8\end{array}$ & $\begin{array}{l}7.9 \\
8.3 \\
8.6 \\
8.4 \\
8.4\end{array}$ & $\begin{array}{l}270 \\
276 \\
276 \\
279 \\
276\end{array}$ & $\begin{array}{l}42370 \\
42376 \\
42388 \\
42391 \\
42397\end{array}$ \\
\hline $\begin{array}{l}22 \\
23 \\
23 \\
23 \\
23\end{array}$ & $\begin{array}{l}6 \\
6 \\
6 \\
6 \\
6\end{array}$ & $\begin{array}{l}1975 \\
1975 \\
1975 \\
1975 \\
1975\end{array}$ & $\begin{array}{r}2238 \\
000 \\
026 \\
130 \\
234\end{array}$ & $\begin{array}{l}36^{\circ} 04.8^{\prime} \\
36^{\circ} 05.9^{\prime} \\
36^{\circ} 06.3^{\prime} \\
36^{\circ} 07.1^{\prime} \\
36^{\circ} 07.9^{\prime}\end{array}$ & $\begin{array}{l}-7^{\circ} 42.8^{\prime} \\
-7^{\circ} 57.9^{\prime} \\
-8^{\circ} 02.7^{\prime} \\
-8^{\circ} 14.6^{\prime} \\
-8^{\circ} 26.5^{\prime}\end{array}$ & $\begin{array}{l}1914.8 \\
1927.0 \\
1930.9 \\
1940.6 \\
1950.2\end{array}$ & $\begin{array}{l}9.0 \\
9.0 \\
9.0 \\
9.0 \\
8.6\end{array}$ & $\begin{array}{l}275 \\
276 \\
275 \\
275 \\
276\end{array}$ & $\begin{array}{l}42403 \\
42406 \\
42410 \\
42415 \\
42418\end{array}$ \\
\hline $\begin{array}{l}23 \\
23 \\
23 \\
23 \\
23\end{array}$ & $\begin{array}{l}6 \\
6 \\
6 \\
6 \\
6\end{array}$ & $\begin{array}{l}1975 \\
1975 \\
1975 \\
1975 \\
1975\end{array}$ & $\begin{array}{l}300 \\
314 \\
420 \\
916 \\
952\end{array}$ & $\begin{array}{l}36^{\circ} 08.3^{\prime} \\
36^{\circ} 08.6^{\prime} \\
36^{\circ} 09.6^{\prime} \\
36^{\circ} 14.8^{\prime} \\
36^{\circ} 15.4^{\prime}\end{array}$ & $\begin{array}{l}-8^{\circ} 31.1^{\prime} \\
-8^{\circ} 33.6^{\prime} \\
-8^{\circ} 45.2^{\prime} \\
-9^{\circ} 34.4^{\prime} \\
-9^{\circ} 40.5^{\prime}\end{array}$ & $\begin{array}{l}1954.0 \\
1956.0 \\
1965.4 \\
2005.5 \\
2010.4\end{array}$ & $\begin{array}{l}8.8 \\
8.6 \\
8.1 \\
8.3 \\
8.2\end{array}$ & $\begin{array}{l}278 \\
276 \\
277 \\
277 \\
278\end{array}$ & $\begin{array}{l}42420 \\
42426 \\
42465 \\
42469 \\
42485\end{array}$ \\
\hline $\begin{array}{l}23 \\
23 \\
23 \\
23 \\
23\end{array}$ & $\begin{array}{l}6 \\
6 \\
6 \\
6 \\
6\end{array}$ & $\begin{array}{l}1975 \\
1975 \\
1975 \\
1975 \\
1975\end{array}$ & $\begin{array}{l}1140 \\
1242 \\
1320 \\
1430 \\
1504\end{array}$ & $\begin{array}{l}36^{\circ} 17.4^{\prime} \\
36^{\circ} 18.7^{\prime} \\
36^{\circ} 19.5^{\prime} \\
36^{\circ} 20.8^{\prime} \\
36^{\circ} 21.5^{\prime}\end{array}$ & $\begin{array}{r}-9^{\circ} 58.6^{\prime} \\
-10^{\circ} 09.0^{\prime} \\
-10^{\circ} 15.6^{\prime} \\
-10^{\circ} 27.7^{\prime} \\
-10^{\circ} 33.3^{\prime}\end{array}$ & $\begin{array}{l}2025.1 \\
2033.6 \\
2039.0 \\
2048.8 \\
2053.4\end{array}$ & $\begin{array}{l}8.2 \\
8.5 \\
8.4 \\
8.1 \\
8.9\end{array}$ & $\begin{array}{l}279 \\
279 \\
278 \\
279 \\
276\end{array}$ & $\begin{array}{l}42496 \\
42502 \\
42514 \\
42520 \\
42524\end{array}$ \\
\hline
\end{tabular}


TABLE 1 - Continued

\begin{tabular}{|c|c|c|c|c|c|c|c|c|c|}
\hline Day & Mon & Year & Time $^{a}$ & Latitude & Longitude & Distance (n. mi.) & Speed $(\mathrm{kt})$ & Course & Regional Mag \\
\hline 23 & 6 & 1975 & 1534 & $36^{\circ} 22.0^{\prime}$ & $-10^{\circ} 38.8^{\prime}$ & 2057.9 & 8.6 & 277 & 42541 \\
\hline 23 & 6 & 1975 & 1722 & $36^{\circ} 23.9^{\prime}$ & $-10^{\circ} 57.8^{\prime}$ & 2073.3 & 8.7 & 279 & 42557 \\
\hline 23 & 6 & 1975 & 1838 & $36^{\circ} 25.6^{\prime}$ & $-11^{\circ} 11.3^{\prime}$ & 2084.3 & 8.5 & 275 & 42571 \\
\hline 23 & 6 & 1975 & 2024 & $36^{\circ} 26.9^{\prime}$ & $-11^{\circ} 30.0^{\prime}$ & 2099.4 & 9.0 & 275 & 42584 \\
\hline 23 & 6 & 1975 & 2150 & $36^{\circ} 28.1^{\prime}$ & $-11^{\circ} 45.9^{\prime}$ & 2112.2 & 8.6 & 276 & 42601 \\
\hline 23 & 6 & 1975 & 2336 & $36^{\circ} 29.7^{\prime}$ & $-12^{\circ} 04.7^{\prime}$ & 2127.4 & 8.7 & 278 & 42606 \\
\hline 24 & 6 & 1975 & 000 & $36^{\circ} 30.2^{\prime}$ & $-12^{\circ} 09.0^{\prime}$ & 2130.9 & 8.6 & 278 & 42614 \\
\hline 24 & 6 & 1975 & 042 & $36^{\circ} 31.0^{\prime}$ & $-12^{\circ} 16.4^{\prime}$ & 2136.9 & 8.6 & 277 & 42632 \\
\hline 24 & 6 & 1975 & 208 & $36^{\circ} 32.6^{\prime}$ & $-12^{\circ} 31.6^{\prime}$ & 2149.2 & 8.8 & 277 & 42636 \\
\hline 24 & 6 & 1975 & 230 & $36^{\circ} 33.0^{\prime}$ & $-12^{\circ} 35.6^{\prime}$ & 2152.5 & 8.9 & 279 & 42652 \\
\hline 24 & 6 & 1975 & 332 & $36^{\circ} 34.5^{\prime}$ & $-12^{\circ} 46.9^{\prime \prime}$ & 2161.7 & 8.5 & 283 & 42658 \\
\hline 24 & 6 & 1975 & 354 & $36^{\circ} 35.2^{\prime}$ & $-12^{\circ} 50.7^{\prime}$ & 2164.8 & 8.1 & 282 & 42682 \\
\hline 24 & 6 & 1975 & 520 & $36^{\circ} 37.6^{\prime}$ & $-13^{\circ} 04.9^{\prime}$ & 2176.4 & 8.6 & 278 & 42699 \\
\hline 24 & 6 & 1975 & 634 & $36^{\circ} 39.1^{\prime}$ & $-13^{\circ} 18.0^{\prime}$ & 2187.1 & 8.5 & 279 & 42709 \\
\hline & 6 & 1975 & 716 & $36^{\circ} 40.0^{\prime}$ & $-13^{\circ} 25.3^{\prime}$ & 2193.0 & 8.6 & 276 & 42752 \\
\hline 24 & 6 & 1975 & 1052 & $36^{\circ} 43.1^{\prime}$ & $-14^{\circ} 03.6^{\prime}$ & 2223.8 & 9.0 & 280 & 42770 \\
\hline 24 & 6 & 1975 & 1154 & $36^{\circ} 44.7^{\prime}$ & $-14^{\circ} 15.0^{\prime}$ & 2233.1 & 8.9 & 279 & 42806 \\
\hline 24 & 6 & 1975 & 1402 & $36^{\circ} 47.7^{\prime}$ & $-14^{\circ} 38.3^{\prime}$ & 2252.0 & 8.6 & 282 & 42815 \\
\hline 24 & 6 & 1975 & 1430 & $36^{\circ} 48.5^{\prime}$ & $-14^{\circ} 43.2^{\prime}$ & 2256.0 & 8.5 & 280 & 42820 \\
\hline 24 & 6 & 1975 & 1466 & $36^{\circ} 48.9^{\prime}$ & $-14^{\circ} 46.0^{\prime}$ & 2258.3 & 8.6 & 278 & 42898 \\
\hline 24 & 6 & 1975 & 1930 & $36^{\circ} 54.7^{\prime}$ & $-15^{\circ} 36.1^{\prime}$ & 2298.8 & 8.4 & 278 & 42929 \\
\hline 24 & 6 & 1975 & 2122 & $36^{\circ} 56.9^{\prime}$ & $-15^{\circ} 55.4^{\prime}$ & 2314.4 & 8.4 & 277 & 42935 \\
\hline 24 & 6 & 1975 & 2145 & $36^{\circ} 57.3^{\prime}$ & $-15^{\circ} 59.4^{\prime}$ & 2317.6 & 8.5 & 275 & 42950 \\
\hline 24 & 6 & 1975 & 2248 & $36^{\circ} 58.1^{\prime}$ & $-16^{\circ} 10.5^{\prime}$ & 2326.5 & 7.8 & 277 & 42966 \\
\hline 24 & 6 & 1975 & 2352 & $36^{\circ} 59.1^{\prime}$ & $-16^{\circ} 20.8^{\prime}$ & 2334.8 & 7.8 & 276 & 42968 \\
\hline 25 & 6 & 1975 & 000 & $36^{\circ} 59.2^{\prime}$ & $-16^{\circ} 22.1^{\prime}$ & 2335.8 & 7.6 & 273 & 42974 \\
\hline 25 & 6 & 1975 & 034 & $36^{\circ} 59.4^{\prime}$ & $-16^{\circ} 27.5^{\prime}$ & 2340.2 & 7.5 & 273 & 42979 \\
\hline 25 & 6 & 1975 & 102 & $36^{\circ} 59.6^{\prime}$ & $-16^{\circ} 31.9^{\prime}$ & 2343,7 & 7.5 & 274 & 43001 \\
\hline 25 & 6 & 1975 & 246 & $37^{\circ} 00.6^{\prime}$ & $-16^{\circ} 48.2^{\prime}$ & 2356.7 & 7.4 & 274 & 43023 \\
\hline 25 & 6 & 1975 & 430 & $37^{\circ} 01.6^{\prime}$ & $-17^{\circ} 04.3^{\prime}$ & 2369.6 & 7.7 & 277 & 43070 \\
\hline 25 & 6 & 1975 & 726 & $37^{\circ} 04.3^{\prime}$ & $-17^{\circ} 32.3^{\prime}$ & 2392.1 & 7.8 & 275 & 43096 \\
\hline 25 & 6 & 1975 & 912 & $37^{\circ} 05.6^{\prime}$ & $-17^{\circ} 49.4^{\prime}$ & 2405.8 & 7.9 & 276 & 43110 \\
\hline 25 & 6 & 1975 & 1002 & $37^{\circ} 06.3^{\prime}$ & $-17^{\circ} 57.6^{\prime}$ & 2412.4 & 7.2 & 274 & 43133 \\
\hline 25 & 6 & 1975 & 1148 & $37^{\circ} 07.1^{\prime}$ & $-18^{\circ} 13.6^{\prime}$ & 2425.2 & 7.9 & 272 & 43146 \\
\hline 25 & 6 & 1975 & 1252 & $37^{\circ} 07.4^{\prime}$ & $-18^{\circ} 24.1^{\prime}$ & 2433.6 & 7.4 & 272 & 43168 \\
\hline 25 & 6 & 1975 & 1436 & $37^{\circ} 07.9^{\prime}$ & $-18^{\circ} 40.1^{\prime}$ & 2446.4 & 8.2 & 272 & 43183 \\
\hline 25 & 6 & 1975 & 1542 & $37^{\circ} 08.2^{\prime}$ & $-18^{\circ} 51.4^{\prime}$ & 2455.4 & 7.9 & 272 & 43192 \\
\hline 25 & 6 & 1975 & 1622 & $37^{\circ} 08.4^{\prime}$ & $-18^{\circ} 58.0^{\prime}$ & 2460.6 & 8.2 & 272 & 43208 \\
\hline 25 & 6 & 1975 & 1730 & $37^{\circ} 08.7^{\prime}$ & $-19^{\circ} 09.7^{\prime}$ & 2470.0 & 6.8 & 273 & 43222 \\
\hline 25 & 6 & 1975 & 1836 & $37^{\circ} 09.1^{\prime}$ & $-19^{\circ} 19.0^{\prime}$ & 2477.4 & 6.8 & 274 & 43225 \\
\hline 25 & 6 & 1975 & 1848 & $37^{\circ} 09.2^{\prime}$ & $-19^{\circ} 20.7^{\prime}$ & 2478.7 & 7.4 & 258 & 43225 \\
\hline 25 & 6 & 1975 & 1852 & $37^{\circ} 09.1^{\prime}$ & $-19^{\circ} 21.3^{\prime}$ & 2479.2 & 7.0 & 252 & 43222 \\
\hline 25 & 6 & 1975 & 1920 & $37^{\circ} 08.1^{\prime}$ & $-19^{\circ} 25.2^{\prime}$ & 2482.5 & 6.8 & 273 & 43235 \\
\hline 25 & 6 & 1975 & 2020 & $37^{\circ} 08.5^{\prime}$ & $-19^{\circ} 33.7^{\prime}$ & 2489.3 & 8.0 & 273 & 43263 \\
\hline 25 & 6 & 1975 & 2202 & $37^{\circ} 09.3^{\prime}$ & $-19^{\circ} 50.7^{\prime}$ & 2502.9 & 7.4 & 272 & 43291 \\
\hline 26 & 6 & 1975 & 000 & $37^{\circ} 09.9^{\prime}$ & $-20^{\circ} 09.0^{\prime}$ & 2517.5 & 7.5 & 273 & 43304 \\
\hline 26 & 6 & 1975 & 050 & $37^{\circ} 10.2^{\prime}$ & $-20^{\circ} 16.8^{\prime}$ & 2523.7 & 7.7 & 273 & 43332 \\
\hline 26 & 6 & 1975 & 236 & $37^{\circ} 10.9^{\prime}$ & $-20^{\circ} 33.9^{\prime}$ & 2537.3 & 7.9 & 273 & 43342 \\
\hline 26 & 6 & 1975 & 310 & $37^{\circ} 11.1^{\prime}$ & $-20^{\circ} 39.5^{\prime}$ & 2541.8 & 7.9 & 273 & 43345 \\
\hline 26 & 6 & 1975 & 324 & $37^{\circ} 11.2^{\prime}$ & $-20^{\circ} 41.8^{\prime}$ & 2543.6 & 7.8 & 277 & 43354 \\
\hline 26 & 6 & 1975 & 348 & $37^{\circ} 11.6^{\prime}$ & $-20^{\circ} 45.7^{\prime}$ & 2546.8 & 8.3 & 273 & 43404 \\
\hline 26 & 6 & 1975 & 632 & $37^{\circ} 12.8^{\prime}$ & $-21^{\circ} 14.3^{\prime}$ & 2569.6 & 8.6 & 273 & 43419 \\
\hline 26 & 6 & 1975 & 718 & $37^{\circ} 13.1^{\prime}$ & $-21^{\circ} 22.6^{\prime}$ & 2576.2 & 8.0 & 274 & 43546 \\
\hline 26 & 6 & 1975 & 1348 & $37^{\circ} 16.4^{\prime}$ & $-22^{\circ} 27.9^{\prime}$ & 2628.3 & 8.3 & 276 & 43569 \\
\hline 26 & 6 & 1975 & 1445 & $37^{\circ} 17.2^{\prime}$ & $-22^{\circ} 37.8^{\prime}$ & 2636.2 & 8.1 & 280 & 43573 \\
\hline 26 & 6 & 1975 & 1454 & $37^{\circ} 17.4^{\prime}$ & $-22^{\circ} 39.3^{\prime}$ & 2637.4 & 8.4 & 276 & 43581 \\
\hline 26 & 6 & 1975 & 1514 & $37^{\circ} 17.7^{\prime}$ & $-22^{\circ} 42.8^{\prime}$ & 2640.2 & 7.7 & 276 & 43652 \\
\hline 26 & 6 & 1975 & 1826 & $37^{\circ} 20.1^{\prime}$ & $-23^{\circ} 13.7^{\prime}$ & 2664.9 & 8.0 & 275 & 43675 \\
\hline 26 & 6 & 1975 & 1926 & $37^{\circ} 20.8^{\prime}$ & $-23^{\circ} 23.7^{\prime}$ & 2672.9 & 7.6 & 274 & 43683 \\
\hline 26 & 6 & 1975 & 1948 & $37^{\circ} 21.0^{\prime}$ & $-23^{\circ} 27.2^{\prime}$ & 2675.7 & 7.5 & 281 & 43725 \\
\hline
\end{tabular}


TABLE 1 - Continued

\begin{tabular}{|c|c|c|c|c|c|c|c|c|c|}
\hline Day & Mon & Year & Time $^{\mathrm{a}}$ & Latitude & Longitude & Distance (n. mi.) & Speed (kt) & Course & Regional Mag \\
\hline 26 & 6 & 1975 & 2120 & $37^{\circ} 23.1^{\prime}$ & $-23^{\circ} 41.4^{\prime}$ & 2687.1 & 9.5 & 277 & 43774 \\
\hline 26 & 6 & 1975 & 2258 & $37^{\circ} 24.9^{\prime}$ & $-24^{\circ} 00.7^{\prime}$ & 2702.6 & 8.5 & 280 & 43807 \\
\hline 27 & 6 & 1975 & 000 & $37^{\circ} 26.5^{\prime}$ & $-24^{\circ} 11.6^{\prime}$ & 2711.4 & 7.2 & 270 & 43808 \\
\hline 27 & 6 & 1975 & 002 & $37^{\circ} 26.5^{\prime}$ & $-24^{\circ} 11.9^{\prime}$ & 2711.6 & 8.5 & 281 & 43830 \\
\hline 27 & 6 & 1975 & 044 & $37^{\circ} 27.6^{\prime}$ & $-24^{\circ} 19.3^{\prime}$ & 2717.6 & 8.2 & 282 & 43864 \\
\hline 27 & 6 & 1975 & 146 & $37^{\circ} 29.4^{\prime}$ & $-24^{\circ} 29.8^{\prime}$ & 2726.1 & 7.7 & 284 & 43882 \\
\hline 27 & 6 & 1975 & 218 & $37^{\circ} 30.4^{\prime}$ & $-24^{\circ} 34.8^{\prime}$ & 2730.2 & 7.8 & 279 & 43932 \\
\hline 27 & 6 & 1975 & 404 & $37^{\circ} 32.6^{\prime}$ & $-24^{\circ} 51.9^{\prime}$ & 2743.9 & 8.5 & 282 & 43965 \\
\hline 27 & 6 & 1975 & 500 & $37^{\circ} 34.3^{\prime}$ & $-25^{\circ} 01.7^{\prime}$ & 2751.9 & 8.5 & 289 & 44026 \\
\hline 27 & 6 & 1975 & 628 & $37^{\circ} 38.3^{\prime}$ & $-25^{\circ} 16.5^{\prime}$ & 2764.3 & 8.5 & 283 & 44036 \\
\hline 27 & 6 & 1975 & 644 & $37^{\circ} 38.8^{\prime}$ & $-25^{\circ} 19.3^{\prime}$ & 2766.6 & 7.5 & 283 & 44048 \\
\hline 27 & 6 & 1975 & 706 & $37^{\circ} 39.4^{\prime}$ & $-25^{\circ} 22.7^{\prime}$ & 2769.3 & 7.5 & 279 & 44055 \\
\hline 27 & 6 & 1975 & 722 & $37^{\circ} 39.7^{\prime}$ & $-25^{\circ} 25.2^{\prime}$ & 2771.3 & 9.9 & 280 & 44067 \\
\hline 27 & 6 & 1975 & 740 & $37^{\circ} 40.2^{\prime}$ & $-25^{\circ} 28.9^{\prime}$ & 2774.3 & 1.3 & 281 & 44098 \\
\hline 27 & 6 & 1975 & 1335 & $37^{\circ} 41.7^{\prime}$ & $-25^{\circ} 38.4^{\prime}$ & 2782.0 & 10.8 & 273 & 44104 \\
\hline 27 & 6 & 1975 & 1346 & $37^{\circ} 41.8^{\prime}$ & $-25^{\circ} 40.9^{\prime}$ & 2783.9 & 8.5 & 276 & 44111 \\
\hline 27 & 6 & 1975 & 1400 & $37^{\circ} 42.0^{\prime}$ & $-25^{\circ} 43.4^{\prime}$ & 2785.9 & 6.0 & 277 & 44114 \\
\hline 27 & 6 & 1975 & 1408 & $37^{\circ} 42.1^{\prime}$ & $-25^{\circ} 44.4^{\prime}$ & 2786.7 & 8.3 & 266 & 44125 \\
\hline 27 & 6 & 1975 & 1446 & $37^{\circ} 41.7^{\prime}$ & $-25^{\circ} 51.0^{\prime}$ & 2792.0 & 8.5 & 271 & 44259 \\
\hline 27 & 6 & 1975 & 2016 & $37^{\circ} 42.3^{\prime}$ & $-26^{\circ} 50.1^{\prime}$ & 2838.7 & 8.7 & 270 & 44305 \\
\hline 27 & 6 & 1975 & 2204 & $37^{\circ} 42.4^{\prime}$ & $-27^{\circ} 09.9^{\prime}$ & 2854.4 & 9.5 & 274 & 44365 \\
\hline 27 & 6 & 1975 & 2356 & $37^{\circ} 43.5^{\prime}$ & $-27^{\circ} 32.2^{\prime}$ & 2872.1 & 9.4 & 270 & 44367 \\
\hline 28 & 6 & 1975 & 000 & $37^{\circ} 43.5^{\prime}$ & $-27^{\circ} 33.0^{\prime}$ & 2872.7 & 8.8 & 271 & 44394 \\
\hline 28 & 6 & 1975 & 058 & $37^{\circ} 43.7^{\prime}$ & $-27^{\circ} 43.8^{\prime}$ & 2881.2 & 10.2 & 270 & 44416 \\
\hline 28 & 6 & 1975 & 140 & $37^{\circ} 43.7^{\prime}$ & $-27^{\circ} 52.8^{\prime}$ & 2888.4 & 8.9 & 271 & 44445 \\
\hline 28 & 6 & 1975 & 240 & $37^{\circ} 43.9^{\prime}$ & $-28^{\circ} 04.0^{\prime}$ & 2897.2 & 8.8 & 272 & 44480 \\
\hline 28 & 6 & 1975 & 350 & $37^{\circ} 44.3^{\prime}$ & $-28^{\circ} 17.0^{\prime}$ & 2907.5 & 9.0 & 271 & 44506 \\
\hline 28 & 6 & 1975 & 442 & $37^{\circ} 44.5^{\prime}$ & $-28^{\circ} 26.9^{\prime}$ & 2915.3 & 9.1 & 270 & 44663 \\
\hline 28 & 6 & 1975 & 958 & $37^{\circ} 44.8^{\prime}$ & $-29^{\circ} 27.4^{\prime}$ & 2963.2 & 8.9 & 274 & 44705 \\
\hline 28 & 6 & 1975 & 1110 & $37^{\circ} 45.6^{\prime}$ & $-29^{\circ} 40.9^{\prime}$ & 2973.9 & 8.9 & 274 & 44742 \\
\hline 28 & 6 & 1975 & 1212 & $37^{\circ} 46.3^{\prime}$ & $-29^{\circ} 52.5^{\prime}$ & 2983.1 & 8.5 & 274 & 44766 \\
\hline 28 & 6 & 1975 & 1256 & $37^{\circ} 46.7^{\prime}$ & $-30^{\circ} 00.4^{\prime}$ & 2989.3 & 9.0 & 271 & 44826 \\
\hline 28 & 6 & 1975 & 1446 & $37^{\circ} 47.1^{\prime}$ & $-30^{\circ} 21.2^{\prime}$ & 3005.8 & 9.3 & 273 & 44861 \\
\hline 28 & 6 & 1975 & 1544 & $37^{\circ} 47.5^{\prime}$ & $-30^{\circ} 32.6^{\prime}$ & 3014.8 & 8.9 & 276 & 44970 \\
\hline 28 & 6 & 1975 & 1832 & $37^{\circ} 50.1^{\prime}$ & $-31^{\circ} 04.0^{\prime}$ & 3039.7 & 8.6 & 272 & 44998 \\
\hline 28 & 6 & 1975 & 1922 & $37^{\circ} 50.3^{\prime}$ & $-31^{\circ} 13.1^{\prime}$ & 3046.9 & 8.6 & 270 & 45010 \\
\hline 28 & 6 & 1975 & 1945 & $37^{\circ} 50.3^{\prime}$ & $-31^{\circ} 17.3^{\prime}$ & 3050.2 & 8.6 & 272 & 45058 \\
\hline 28 & 6 & 1975 & 2108 & $37^{\circ} 50.8^{\prime}$ & $-31^{\circ} 32.4^{\prime}$ & 3062.2 & 9.0 & 272 & 45122 \\
\hline 28 & 6 & 1975 & 2256 & $37^{\circ} 51.4^{\prime}$ & $-31^{\circ} 52.8^{\prime}$ & 3078.3 & 9.5 & 272 & 45133 \\
\hline 28 & 6 & 1975 & 2312 & $37^{\circ} 51.5^{\prime}$ & $-31^{\circ} 56.0^{\prime}$ & 3080.8 & 8.8 & 272 & 45161 \\
\hline 29 & 6 & 1975 & 000 & $37^{\circ} 51.7^{\prime}$ & $-32^{\circ} 04.9^{\prime}$ & 3087.9 & 8.9 & 271 & 45193 \\
\hline 29 & 6 & 1975 & 054 & $37^{\circ} 51.9^{\prime}$ & $-32^{\circ} 15.0^{\prime}$ & 3095.8 & 8.6 & 272 & 45208 \\
\hline 29 & 6 & 1975 & 120 & $37^{\circ} 52.0^{\prime}$ & $-32^{\circ} 19.7^{\prime}$ & 3099.5 & 8.4 & 274 & 45226 \\
\hline 29 & 6 & 1975 & 150 & $37^{\circ} 52.3^{\prime}$ & $-32^{\circ} 25.0^{\prime}$ & 3103.7 & 9.3 & 274 & 45344 \\
\hline 29 & 6 & 1975 & 440 & $37^{\circ} 54.2^{\prime}$ & $-32^{\circ} 58.2^{\prime}$ & 3130.0 & 8.6 & 273 & 45370 \\
\hline & 6 & 1975 & & $37^{\circ} 54.5^{\prime}$ & $-33^{\circ} 05.8^{\prime}$ & 3136.0 & 9.2 & 276 & 45418 \\
\hline 29 & 6 & 1975 & 628 & $37^{\circ} 55.5^{\prime}$ & $-33^{\circ} 18.6^{\prime}$ & 3146.2 & 9.3 & 275 & 45456 \\
\hline 29 & 6 & 1975 & 720 & $37^{\circ} 56.2^{\prime}$ & $-33^{\circ} 28.8^{\prime}$ & 3154.2 & 9.1 & 269 & 45491 \\
\hline 29 & 6 & 1975 & 818 & $37^{\circ} 56.1^{\prime}$ & $-33^{\circ} 40.0^{\prime}$ & 3163.1 & 8.8 & 278 & 45608 \\
\hline 29 & 6 & 1975 & 1052 & $37^{\circ} 59.2^{\prime}$ & $-34^{\circ} 08.5^{\prime}$ & 3185.7 & 9.0 & 277 & 45666 \\
\hline 29 & 6 & 1975 & 1208 & $38^{\circ} 00.6^{\prime}$ & $-34^{\circ} 22.8^{\prime}$ & 3197.1 & 8.9 & 276 & 45711 \\
\hline 29 & 6 & 1975 & 1308 & $38^{\circ} 01.6^{\prime}$ & $-34^{\circ} 34.0^{\prime}$ & 3206.0 & 9.1 & 275 & 45790 \\
\hline 29 & 6 & 1975 & 1454 & $38^{\circ} 03.1^{\prime}$ & $-34^{\circ} 54.3^{\prime}$ & 3222.0 & 9.4 & 274 & 45895 \\
\hline 29 & 6 & 1975 & 1712 & $38^{\circ} 04.5^{\prime}$ & $-35^{\circ} 21.7^{\prime}$ & 3243.7 & 9.4 & 271 & 45915 \\
\hline 29 & 6 & 1975 & 1740 & $38^{\circ} 04.6^{\prime}$ & $-35^{\circ} 27.3^{\prime}$ & 3248.1 & 9.1 & 275 & 45995 \\
\hline 29 & 6 & 1975 & 1926 & $38^{\circ} 05.9^{\prime}$ & $-35^{\circ} 47.6^{\prime}$ & 3264.1 & 9.6 & 274 & 46215 \\
\hline 30 & 6 & 1975 & 000 & $38^{\circ} 08.7^{\prime}$ & $-36^{\circ} 43.5^{\prime}$ & 3308.2 & 9.5 & 276 & 46220 \\
\hline 30 & 6 & 1975 & 006 & $38^{\circ} 08.8^{\prime}$ & $-36^{\circ} 44.7^{\prime}$ & 3309.1 & 9.1 & 272 & 46266 \\
\hline 30 & 6 & 1975 & 108 & $38^{\circ} 09.2^{\prime}$ & $-36^{\circ} 56.6^{\prime}$ & 3318.5 & 9.0 & 282 & 46365 \\
\hline 30 & 6 & 1975 & 256 & $38^{\circ} 12.5^{\prime}$ & $-37^{\circ} 16.7^{\prime}$ & 3334.6 & 10.4 & 271 & 46420 \\
\hline
\end{tabular}


TABLE 1 - Continued

\begin{tabular}{|c|c|c|c|c|c|c|c|c|c|}
\hline Day & Mon & Year & Time $^{\mathrm{a}}$ & Latitude & Longitude & Distance (n. mi.) & Speed $(k t)$ & Course & Regional Mag \\
\hline 30 & 6 & 1975 & 402 & $38^{\circ} 12.6^{\prime}$ & $-37^{\circ} 31.3^{\prime}$ & 3346.1 & 9.0 & 282 & 46512 \\
\hline 30 & 6 & 1975 & 540 & $38^{\circ} 15.7^{\prime}$ & $-37^{\circ} 49.6^{\prime}$ & 3360.8 & 8.9 & 279 & 46603 \\
\hline 30 & 6 & 1975 & 724 & $38^{\circ} 18.0^{\prime}$ & $-38^{\circ} 09.1^{\prime}$ & 3376.3 & 9.4 & 277 & 46647 \\
\hline 30 & 6 & 1975 & 814 & $38^{\circ} 18.9^{\prime}$ & $-38^{\circ} 19.0^{\prime}$ & 3384.1 & 9.4 & 276 & 46738 \\
\hline 30 & 6 & 1975 & 956 & $38^{\circ} 20.7^{\prime}$ & $-38^{\circ} 39.2^{\prime}$ & 3400.0 & 8.7 & 283 & 46817 \\
\hline 30 & 6 & 1975 & 1120 & $38^{\circ} 23.4^{\prime}$ & $-38^{\circ} 54.3^{\prime}$ & 3412.2 & 9.1 & 280 & 46916 \\
\hline 30 & 6 & 1975 & 1306 & $38^{\circ} 26.1^{\prime}$ & $-39^{\circ} 14.6^{\prime}$ & 3428.3 & 8.5 & 278 & 46937 \\
\hline 30 & 6 & 1975 & 1330 & $38^{\circ} 26.6^{\prime}$ & $-39^{\circ} 18.9^{\prime}$ & 3431.7 & 8.6 & 272 & 46964 \\
\hline 30 & 6 & 1975 & 1406 & $38^{\circ} 26.8^{\prime}$ & $-39^{\circ} 25.5^{\prime}$ & 3436.9 & 9.1 & 272 & 47050 \\
\hline 30 & 6 & 1975 & 1552 & $38^{\circ} 27.5^{\prime}$ & $-39^{\circ} 46.0^{\prime}$ & 3453.0 & 8.9 & 268 & 47357 \\
\hline 30 & 6 & 1975 & 2252 & $38^{\circ} 25.8^{\prime}$ & $-41^{\circ} 05.5^{\prime}$ & 3515.3 & 10.0 & 261 & 47376 \\
\hline 30 & 6 & 1975 & 2320 & $38^{\circ} 25.1^{\prime}$ & $-41^{\circ} 11.4^{\prime}$ & 3519.9 & 9.7 & 270 & 47410 \\
\hline 1 & 7 & 1975 & 000 & $38^{\circ} 25.1^{\prime}$ & $-41^{\circ} 19.7^{\prime}$ & 3526.4 & 9.8 & 271 & 47466 \\
\hline 1 & 7 & 1975 & 104 & $38^{\circ} 25.2^{\prime}$ & $-41^{\circ} 33.1^{\prime}$ & 3536.9 & 9.8 & 270 & 47488 \\
\hline 1 & 7 & 1975 & 130 & $38^{\circ} 25.2^{\prime}$ & $-41^{\circ} 38.5^{\prime}$ & 3541.2 & 9.7 & 275 & 47522 \\
\hline 1 & 7 & 1975 & 206 & $38^{\circ} 25.7^{\prime}$ & $-41^{\circ} 45.9^{\prime}$ & 3547.0 & 9.7 & 278 & 47799 \\
\hline 1 & 7 & 1975 & 632 & $38^{\circ} 32.0^{\prime}$ & $-42^{\circ} 40.4^{\prime}$ & 3590.1 & 8.9 & 277 & 47901 \\
\hline 1 & 7 & 1975 & 820 & $38^{\circ} 34.0^{\prime}$ & $-43^{\circ} 00.8^{\prime}$ & 3606.2 & 9.4 & 274 & 47924 \\
\hline 1 & 7 & 1975 & 845 & $38^{\circ} 34.3^{\prime}$ & $-43^{\circ} 05.8^{\prime}$ & 3610.1 & 9.4 & 270 & 47937 \\
\hline 1 & 7 & 1975 & 900 & $38^{\circ} 34.3^{\prime}$ & $-43^{\circ} 08.8^{\prime}$ & 3612.5 & 9.0 & 271 & 48029 \\
\hline 1 & 7 & 1975 & 1050 & $38^{\circ} 34.6^{\prime}$ & $-43^{\circ} 29.8^{\prime}$ & 3628.9 & 9.1 & 270 & 48102 \\
\hline 1 & 7 & 1975 & 1218 & $38^{\circ} 34.5^{\prime}$ & $-43^{\circ} 46.8^{\prime}$ & 3642.2 & 9.1 & 268 & 48183 \\
\hline 1 & 7 & 1975 & 1400 & $38^{\circ} 33.9^{\prime}$ & $-44^{\circ} 06.5^{\prime}$ & 3657.6 & 9.1 & 271 & 48296 \\
\hline 1 & 7 & 1975 & 1610 & $38^{\circ} 34.3^{\prime}$ & $-44^{\circ} 31.6^{\prime}$ & 3677.2 & 9.0 & 272 & 48324 \\
\hline 1 & 7 & 1975 & 1642 & $38^{\circ} 34.5^{\prime}$ & $-44^{\circ} 37.7^{\prime}$ & 3682.0 & 8.9 & 274 & 48380 \\
\hline 1 & 7 & 1975 & 1744 & $38^{\circ} 35.1^{\prime}$ & $-44^{\circ} 49.4^{\prime}$ & 3691.1 & 9.0 & 276 & 48481 \\
\hline 1 & 7 & 1975 & 1930 & $38^{\circ} 36.7^{\prime}$ & $-45^{\circ} 09.6^{\prime}$ & 3707.0 & 8.2 & 275 & 48516 \\
\hline 1 & 7 & 1975 & 2010 & $38^{\circ} 37.2^{\prime}$ & $-45^{\circ} 16.6^{\prime}$ & 3712.5 & 9.3 & 276 & 48622 \\
\hline 1 & 7 & 1975 & 2156 & $38^{\circ} 39.0^{\prime}$ & $-45^{\circ} 374^{\prime}$ & 3728.8 & 8.6 & 273 & 48732 \\
\hline 2 & 7 & 1975 & 000 & $38^{\circ} 40.0^{\prime}$ & $-46^{\circ} 00.2^{\prime}$ & 3746.7 & 8.5 & 273 & 48746 \\
\hline 2 & 7 & 1975 & 016 & $38^{\circ} 40.1^{\prime}$ & $-46^{\circ} 03.1^{\prime}$ & 3748.9 & 9.4 & 276 & 48914 \\
\hline 2 & 7 & 1975 & 302 & $38^{\circ} 42.7^{\prime}$ & $-46^{\circ} 36.2^{\prime}$ & 3774.9 & 9.7 & 278 & 48963 \\
\hline 2 & 7 & 1975 & 346 & $38^{\circ} 43.7^{\prime}$ & $-46^{\circ} 45.2^{\prime}$ & 3782.0 & 10.3 & 275 & 48991 \\
\hline 2 & 7 & 1975 & 412 & $38^{\circ} 44.1^{\prime}$ & $-46^{\circ} 50.9^{\prime}$ & 3786.5 & 9.4 & 282 & 49082 \\
\hline 2 & 7 & 1975 & 532 & $38^{\circ} 46.7^{\prime}$ & $-47^{\circ} 06.7^{\prime}$ & 3799.1 & 8.3 & 281 & 49196 \\
\hline 2 & 7 & 1975 & 728 & $38^{\circ} 49.7^{\prime}$ & $-47^{\circ} 26.8^{\prime}$ & 3815.0 & 7.4 & 282 & 49417 \\
\hline 2 & 7 & 1975 & 1132 & $38^{\circ} 56.0^{\prime}$ & $-48^{\circ} 04.8^{\prime}$ & 3845.3 & 7.7 & 282 & 49439 \\
\hline 2 & 7 & 1975 & 1155 & $38^{\circ} 56.6^{\prime}$ & $-48^{\circ} 08.5^{\prime}$ & 3848.2 & 7.5 & 271 & 49499 \\
\hline 2 & 7 & 1975 & 1316 & $38^{\circ} 56.7^{\prime}$ & $-48^{\circ} 21.5^{\prime}$ & 3858.3 & 7.1 & 265 & 49535 \\
\hline 2 & 7 & 1975 & 1414 & $38^{\circ} 56.1^{\prime}$ & $-48^{\circ} 30.3^{\prime}$ & 3865.2 & 7.6 & 266 & 49593 \\
\hline 2 & 7 & 1975 & 1538 & $38^{\circ} 55.3^{\prime}$ & $-48^{\circ} 44.0^{\prime}$ & 3875.9 & 7.5 & 272 & 49611 \\
\hline 2 & 7 & 1975 & 1601 & $38^{\circ} 55.4^{\prime}$ & $-48^{\circ} 47.7^{\prime}$ & 3878.7 & 7.2 & 233 & 49614 \\
\hline 2 & 7 & 1975 & 1714 & $38^{\circ} 50.2^{\prime}$ & $-48^{\circ} 56.7^{\prime}$ & 3887.5 & 7.8 & 231 & 49614 \\
\hline 2 & 7 & 1975 & 1838 & $38^{\circ} 43.3^{\prime}$ & $-49^{\circ} 07.6^{\prime}$ & 3898.4 & 6.4 & 224 & 49611 \\
\hline 2 & 7 & 1975 & 1900 & $38^{\circ} 41.6^{\prime}$ & $-49^{\circ} 09.7^{\prime}$ & 3900.8 & 6.0 & 214 & 49587 \\
\hline 2 & 7 & 1975 & 2026 & $38^{\circ} 34.5^{\prime}$ & $-49^{\circ} 15.8^{\prime}$ & 3909.3 & 7.1 & 207 & 49571 \\
\hline 2 & 7 & 1975 & 2100 & $38^{\circ} 30.9^{\prime}$ & $-49^{\circ} 18.1^{\prime}$ & 3913.3 & 5.5 & 224 & 49559 \\
\hline 2 & 7 & 1975 & 2246 & $38^{\circ} 24.0^{\prime}$ & $-49^{\circ} 26.7^{\prime}$ & 3923.0 & 6.9 & 239 & 49571 \\
\hline 3 & 7 & 1975 & 000 & $38^{\circ} 19.7^{\prime}$ & $-49^{\circ} 36.0^{\prime}$ & 3931.5 & 6.9 & 239 & 49591 \\
\hline 3 & 7 & 1975 & 216 & $38^{\circ} 11.7^{\prime}$ & $-49^{\circ} 53.2^{\prime}$ & 3947.2 & 7.3 & 241 & 49600 \\
\hline 3 & 7 & 1975 & 302 & $38^{\circ} 09.0^{\prime}$ & $-49^{\circ} 59.4^{\prime}$ & 3952.7 & 7.1 & 245 & 49615 \\
\hline 3 & 7 & 1975 & 400 & $38^{\circ} 06.1^{\prime}$ & $-50^{\circ} 07.3^{\prime}$ & 3959.6 & 8.4 & 228 & 49613 \\
\hline 3 & 7 & 1975 & 430 & $38^{\circ} 03.3^{\prime}$ & $-50^{\circ} 11.3^{\prime}$ & 3963.8 & 7.1 & 264 & 49637 \\
\hline 3 & 7 & 1975 & 510 & $38^{\circ} 02.8^{\prime}$ & $-50^{\circ} 17.3^{\prime}$ & 3968.5 & 6.7 & 248 & 49656 \\
\hline 3 & 7 & 1975 & 610 & $38^{\circ} 00.3^{\prime}$ & $-50^{\circ} 25.2^{\prime}$ & 3975.3 & 7.1 & 247 & 49664 \\
\hline 3 & 7 & 1975 & 636 & $37^{\circ} 59.1^{\prime}$ & $-50^{\circ} 28.8^{\prime}$ & 3978.3 & 6.8 & 249 & 49670 \\
\hline 3 & 7 & 1975 & 656 & $37^{\circ} 58.3^{\prime}$ & $-50^{\circ} 31.5^{\prime}$ & 3980.6 & 8.7 & 290 & 49675 \\
\hline 3 & 7 & 1975 & 700 & $37^{\circ} 58.5^{\prime}$ & $-50^{\circ} 32.2^{\prime}$ & 3981.2 & 7.1 & 236 & 49682 \\
\hline 3 & 7 & 1975 & 824 & $37^{\circ} 53.0^{\prime}$ & $-50^{\circ} 42.6^{\prime}$ & 3991.1 & 7.1 & 238 & 49687 \\
\hline 3 & 7 & 1975 & 856 & $37^{\circ} 51.0^{\prime}$ & $-50^{\circ} 46.7^{\prime}$ & 3994.9 & 7.2 & 241 & 49691 \\
\hline
\end{tabular}


TABLE 1 - Continued

\begin{tabular}{|c|c|c|c|c|c|c|c|c|c|}
\hline Day & Mon & Year & Time $^{\mathrm{a}}$ & Latitude & Longitude & Distance (n. mi.) & Speed $(k t)$ & Course & Regional Mag \\
\hline 3 & 7 & 1975 & 920 & $37^{\circ} 49.6^{\prime}$ & $-50^{\circ} 49.9^{\prime}$ & 3997.8 & 7.2 & 236 & 49699 \\
\hline 3 & 7 & 1975 & 1042 & $37^{\circ} 44.2^{\prime}$ & $-51^{\circ} 00.2^{\prime}$ & 4007.5 & 7.5 & 236 & 49709 \\
\hline 3 & 7 & 1975 & 1228 & $37^{\circ} 36.8^{\prime}$ & $-51^{\circ} 14.2^{\prime}$ & 4020.9 & 7.5 & 233 & 49710 \\
\hline 3 & 7 & 1975 & 1326 & $37^{\circ} 32.4^{\prime}$ & $-51^{\circ} 21.5^{\prime}$ & 4028.1 & 6.9 & 229 & 49707 \\
\hline 3 & 7 & 1975 & 1414 & $37^{\circ} 28.8^{\prime}$ & $-51^{\circ} 26.8^{\prime}$ & 4033.7 & 6.7 & 227 & 49685 \\
\hline 3 & 7 & 1975 & 1748 & $37^{\circ} 12.3^{\prime}$ & $-51^{\circ} 48.8^{\prime}$ & 4057.7 & 6.0 & 230 & 49681 \\
\hline 3 & 7 & 1975 & 1934 & $37^{\circ} 05.5^{\prime}$ & $-51^{\circ} 59.1^{\prime}$ & 4068.4 & 5.5 & 224 & 49679 \\
\hline 3 & 7 & 1975 & 1954 & $37^{\circ} 04.2^{\prime}$ & $-52^{\circ} 00.7^{\prime}$ & 4070.2 & 5.9 & 230 & 49674 \\
\hline 3 & 7 & 1975 & 2152 & $36^{\circ} 56.8^{\prime}$ & $-52^{\circ} 11.9^{\prime}$ & 4081.8 & 6.5 & 235 & 49680 \\
\hline 3 & 7 & 1975 & 2340 & $36^{\circ} 50.2^{\prime}$ & $-52^{\circ} 23.9^{\prime}$ & 4093.4 & 5.8 & 236 & 49681 \\
\hline 4 & 7 & 1975 & 000 & $36^{\circ} 49.1^{\prime}$ & $-52^{\circ} 25.9^{\prime}$ & 4095.4 & 5.9 & 234 & 49682 \\
\hline 4 & 7 & 1975 & 026 & $36^{\circ} 47.6^{\prime}$ & $-52^{\circ} 28.5^{\prime}$ & 4097.9 & 6.1 & 231 & 49680 \\
\hline 4 & 7 & 1975 & 126 & $36^{\circ} 43.8^{\prime}$ & $-52^{\circ} 34.4^{\prime}$ & 4104.0 & 6.3 & 231 & 49679 \\
\hline 4 & 7 & 1975 & 212 & $36^{\circ} 40.8^{\prime}$ & $-52^{\circ} 39.1^{\prime}$ & 4108.8 & 6.5 & 234 & 49682 \\
\hline 4 & 7 & 1975 & 420 & $36^{\circ} 32.6^{\prime}$ & $-52^{\circ} 53.2^{\prime}$ & 4122.8 & 6.7 & 235 & 49686 \\
\hline 4 & 7 & 1975 & 546 & $36^{\circ} 27.1^{\prime}$ & $-53^{\circ} 03.1^{\prime}$ & 4132.5 & 6.7 & 238 & 49695 \\
\hline 4 & 7 & 1975 & 730 & $36^{\circ} 20.9^{\prime}$ & $-53^{\circ} 15.4^{\prime}$ & 4144.2 & 7.2 & 239 & 49712 \\
\hline 4 & 7 & 1975 & 946 & $36^{\circ} 12.6^{\prime}$ & $-53^{\circ} 32.7^{\prime}$ & 4160.4 & 7.5 & 236 & 49718 \\
\hline 4 & 7 & 1975 & 1134 & $36^{\circ} 05.1^{\prime}$ & $-53^{\circ} 46.6^{\prime}$ & 4173.9 & 7.7 & 231 & 49708 \\
\hline 4 & 7 & 1975 & 1422 & $35^{\circ} 51.4^{\prime}$ & $-54^{\circ} 07.3^{\prime}$ & 4195.5 & 7.3 & 230 & 49703 \\
\hline 4 & 7 & 1975 & 1510 & $35^{\circ} 47.6^{\prime}$ & $-54^{\circ} 12.8^{\prime}$ & 4201.4 & 7.4 & 233 & 49702 \\
\hline 4 & 7 & 1975 & 1610 & $35^{\circ} 43.1^{\prime}$ & $-54^{\circ} 20.1^{\prime}$ & 4208.8 & 7.1 & 241 & 49708 \\
\hline 4 & 7 & 1975 & 1652 & $35^{\circ} 40.7^{\prime}$ & $-54^{\circ} 25.5^{\prime}$ & 4213.8 & 7.8 & 220 & 49700 \\
\hline 4 & 7 & 1975 & 1718 & $35^{\circ} 38.1^{\prime}$ & $-54^{\circ} 28.2^{\prime}$ & 4217.2 & 7.8 & 224 & 49676 \\
\hline 4 & 7 & 1975 & 1906 & $35^{\circ} 28.0^{\prime}$ & $-54^{\circ} 40.3^{\prime}$ & 4231.3 & 7.8 & 226 & 49660 \\
\hline 4 & 7 & 1975 & 2030 & $35^{\circ} 20.4^{\prime}$ & $-54^{\circ} 49.9^{\prime}$ & 4242.2 & 7.7 & 228 & 49656 \\
\hline 4 & 7 & 1975 & 2100 & $35^{\circ} 17.8^{\prime}$ & $-54^{\circ} 53.4^{\prime}$ & 4246.1 & 7.9 & 230 & 49644 \\
\hline 4 & 7 & 1975 & 2244 & $35^{\circ} 09.0^{\prime}$ & $-55^{\circ} 06.2^{\prime}$ & 4259.8 & 7.6 & 230 & 49643 \\
\hline 4 & 7 & 1975 & 2300 & $35^{\circ} 07.7^{\prime}$ & $-55^{\circ} 08.1^{\prime}$ & 4261.8 & 7.9 & 258 & 49673 \\
\hline 5 & 7 & 1975 & 000 & $35^{\circ} 06.0^{\prime}$ & $-55^{\circ} 17.5^{\prime}$ & 4269.7 & 7.8 & 257 & 49740 \\
\hline 5 & 7 & 1975 & 213 & $35^{\circ} 02.2^{\prime}$ & $-55^{\circ} 38.1^{\prime}$ & 4286.9 & 7.8 & 244 & 49741 \\
\hline 5 & 7 & 1975 & 220 & $35^{\circ} 01.8^{\prime}$ & $-55^{\circ} 39.1^{\prime}$ & 4287.9 & 7.5 & 243 & 49751 \\
\hline 5 & 7 & 1975 & 312 & $34^{\circ} 58.9^{\prime}$ & $-55^{\circ} 46.2^{\prime}$ & 4294.4 & 7.8 & 245 & 49761 \\
\hline 5 & 7 & 1975 & 358 & $34^{\circ} 56.4^{\prime}$ & $-55^{\circ} 52.8^{\prime}$ & 4300.3 & 7.8 & 243 & 49775 \\
\hline 5 & 7 & 1975 & 516 & $34^{\circ} 51.8^{\prime}$ & $-56^{\circ} 03.8^{\prime}$ & 4310.4 & 8.3 & 240 & 49779 \\
\hline 5 & 7 & 1975 & 542 & $34^{\circ} 50.0^{\prime}$ & $-56^{\circ} 07.6^{\prime}$ & 4314.0 & 8.3 & 240 & 49784 \\
\hline 5 & 7 & 1975 & 638 & $34^{\circ} 46.1^{\prime}$ & $-56^{\circ} 15.7^{\prime}$ & 4321.7 & 8.5 & 240 & 49787 \\
\hline 5 & 7 & 1975 & 702 & $34^{\circ} 44.4^{\prime}$ & $-56^{\circ} 19.3^{\prime}$ & 4325.2 & 8.5 & 240 & 49796 \\
\hline 5 & 7 & 1975 & 826 & $34^{\circ} 38.4^{\prime}$ & $-56^{\circ} 31.8^{\prime}$ & 4337.1 & 8.2 & 239 & 49799 \\
\hline 5 & 7 & 1975 & 852 & $34^{\circ} 36.6^{\prime}$ & $-56^{\circ} 35.5^{\prime}$ & 4340.6 & 9.5 & 247 & 49828 \\
\hline 5 & 7 & 1975 & 1024 & $34^{\circ} 30.9^{\prime}$ & $-56^{\circ} 51.8^{\prime}$ & 4355.2 & 10.0 & 113 & 49808 \\
\hline 5 & 7 & 1975 & 1038 & $34^{\circ} 30.0^{\prime}$ & $-56^{\circ} 49.2^{\prime}$ & 4357.5 & 8.6 & 113 & 49660 \\
\hline 5 & 7 & 1975 & 1240 & $34^{\circ} 23.2^{\prime}$ & $-56^{\circ} 29.8^{\prime}$ & 4374.9 & 9.8 & 109 & 49628 \\
\hline 5 & 7 & 1975 & 1305 & $34^{\circ} 21.9^{\prime}$ & $-56^{\circ} 25.1^{\prime}$ & 4379.0 & 9.3 & 298 & 49653 \\
\hline 5 & 7 & 1975 & 1323 & $34^{\circ} 23.2^{\prime}$ & $-56^{\circ} 28.1^{\prime}$ & 4381.8 & 6.6 & 298 & 49687 \\
\hline 5 & 7 & 1975 & 1358 & $34^{\circ} 25.0^{\prime}$ & $-56^{\circ} 32.2^{\prime}$ & 4385.6 & 0.0 & 90 & 49686 \\
\hline 9 & 7 & 1975 & 830 & $34^{\circ} 25.0^{\prime}$ & $-56^{\circ} 32.2^{\prime}$ & 4385.6 & 5.7 & 29 & 49718 \\
\hline 9 & 7 & 1975 & 942 & $34^{\circ} 31.0^{\prime}$ & $-56^{\circ} 28.2^{\prime}$ & 4392.5 & 8.7 & 29 & 49749 \\
\hline 9 & 7 & 1975 & 1030 & $34^{\circ} 37.1^{\prime}$ & $-56^{\circ} 24.1^{\prime}$ & 4399.4 & 9.3 & 28 & 49855 \\
\hline 9 & 7 & 1975 & 1258 & $34^{\circ} 57.4^{\prime}$ & $-56^{\circ} 11.0^{\prime}$ & 4422.4 & 10.0 & 33 & 49888 \\
\hline 9 & 7 & 1975 & 1350 & $35^{\circ} 04.7^{\prime}$ & $-56^{\circ} 05.3^{\prime}$ & 4431.1 & 11.1 & 29 & 49932 \\
\hline 9 & 7 & 1975 & 1446 & $35^{\circ} 13.7^{\prime}$ & $-55^{\circ} 59.1^{\prime}$ & 4441.4 & 9.2 & 32 & 49962 \\
\hline 9 & 7 & 1975 & 1538 & $35^{\circ} 20.4^{\prime}$ & $-55^{\circ} 53.9^{\prime}$ & 4449.3 & 9.4 & 29 & 50053 \\
\hline 9 & 7 & 1975 & 1758 & $35^{\circ} 39.6^{\prime}$ & $-55^{\circ} 40.6^{\prime}$ & 4471.4 & 9.4 & 30 & 50080 \\
\hline 9 & 7 & 1975 & 1840 & $35^{\circ} 45.3^{\prime}$ & $-55^{\circ} 36.6^{\prime}$ & 4477.9 & 8.9 & 30 & 50120 \\
\hline 9 & 7 & 1975 & 1946 & $35^{\circ} 53.8^{\prime}$ & $-55^{\circ} 30.5^{\prime}$ & 4487.8 & 8.8 & 26 & 50197 \\
\hline 9 & 7 & 1975 & 2142 & $36^{\circ} 09.1^{\prime}$ & $-55^{\circ} 21.1^{\prime}$ & 4504.8 & 8.7 & 27 & 50253 \\
\hline 9 & 7 & 1975 & 2310 & $36^{\circ} 20.5^{\prime}$ & $-55^{\circ} 13.9^{\prime}$ & 4517.6 & 8.9 & 29 & 50265 \\
\hline 9 & 7 & 1975 & 2330 & $36^{\circ} 23.1^{\prime}$ & $-55^{\circ} 12.1^{\prime}$ & 4520.6 & 8.9 & 26 & 50285 \\
\hline 10 & 7 & 1975 & 000 & $36^{\circ} 27.1^{\prime}$ & $-55^{\circ} 09.7^{\prime}$ & 4525.1 & 8.9 & 26 & 50321 \\
\hline
\end{tabular}


TABLE 1 - Continued

\begin{tabular}{|c|c|c|c|c|c|c|c|c|c|}
\hline Day & Mon & Year & Time $^{\mathrm{a}}$ & Latitude & Longitude & Distance (n. mi.) & Speed (kt) & Course & Regional Mag \\
\hline 10 & 7 & 1975 & 054 & $36^{\circ} 34.3^{\prime}$ & $-55^{\circ} 05.4^{\prime}$ & 4533.0 & 9.8 & 29 & 50357 \\
\hline 10 & 7 & 1975 & 150 & $36^{\circ} 42.3^{\prime}$ & $-54^{\circ} 59.8^{\prime}$ & 4542.2 & 9.4 & 27 & 50364 \\
\hline 10 & 7 & 1975 & 200 & $36^{\circ} 43.7^{\prime}$ & $-54^{\circ} 58.9^{\prime}$ & 4543.8 & 9.6 & 30 & 50389 \\
\hline 10 & 7 & 1975 & 242 & $36^{\circ} 49.5^{\prime}$ & $-54^{\circ} 54.7^{\prime}$ & 4550.5 & 8.6 & 28 & 50423 \\
\hline 10 & 7 & 1975 & 340 & $36^{\circ} 56.8^{\prime}$ & $-54^{\circ} 49.8^{\prime}$ & 4558.8 & 10.5 & 30 & 50466 \\
\hline 10 & 7 & 1975 & 446 & $37^{\circ} 6.8^{\prime}$ & $-54^{\circ} 42.6^{\prime}$ & 4570.3 & 9.1 & 31 & 50522 \\
\hline 10 & 7 & 1975 & 632 & $37^{\circ} 20.6^{\prime}$ & $-54^{\circ} 32.1^{\prime}$ & 4586.4 & 8.7 & 33 & 50555 \\
\hline 10 & 7 & 1975 & 742 & $37^{\circ} 29.2^{\prime}$ & $-54^{\circ} 25.2^{\prime}$ & 4596.6 & 8.6 & 30 & 50667 \\
\hline 10 & 7 & 1975 & 1124 & $37^{\circ} 56.8^{\prime}$ & $-54^{\circ} 04.9^{\prime}$ & 4628.6 & 9.1 & 36 & 50684 \\
\hline 10 & 7 & 1975 & 1208 & $38^{\circ} 02.2^{\prime}$ & $-53^{\circ} 59.9^{\prime}$ & 4635.3 & 9.6 & 38 & 50721 \\
\hline 10 & 7 & 1975 & 1356 & $38^{\circ} 15.8^{\prime}$ & $-53^{\circ} 46.4^{\prime}$ & 4652.5 & 10.4 & 42 & 50728 \\
\hline 10 & 7 & 1975 & 1425 & $38^{\circ} 19.5^{\prime}$ & $-53^{\circ} 42.1^{\prime}$ & 4657.5 & 10.2 & 37 & 50752 \\
\hline 10 & 7 & 1975 & 1528 & $38^{\circ} 28.0^{\prime}$ & $-53^{\circ} 33.9^{\prime}$ & 4668.2 & 9.8 & 37 & 50763 \\
\hline 10 & 7 & 1975 & 1558 & $38^{\circ} 31.9^{\prime}$ & $-53^{\circ} 30.1^{\prime}$ & 4673.1 & 10.6 & 35 & 50772 \\
\hline 10 & 7 & 1975 & 1618 & $38^{\circ} 34.8^{\prime}$ & $-53^{\circ} 27.5^{\prime}$ & 4676.6 & 10.5 & 34 & 50812 \\
\hline 10 & 7 & 1975 & 1744 & $38^{\circ} 47.2^{\prime}$ & $-53^{\circ} 16.7^{\prime}$ & 4691.6 & 11.9 & 40 & 50820 \\
\hline 10 & 7 & 1975 & 1809 & $38^{\circ} 51.0^{\prime}$ & $-53^{\circ} 12.6^{\prime}$ & 4696.6 & 8.6 & 354 & 50868 \\
\hline 10 & 7 & 1975 & 1852 & $38^{\circ} 57.1^{\prime}$ & $-53^{\circ} 13.4^{\prime}$ & 4702.7 & 8.1 & 348 & 50989 \\
\hline 10 & 7 & 1975 & 2040 & $39^{\circ} 11.3^{\prime}$ & $-53^{\circ} 17.2^{\prime}$ & 4717.2 & 9.2 & 341 & 51016 \\
\hline 10 & 7 & 1975 & 2100 & $39^{\circ} 14.2^{\prime}$ & $-53^{\circ} 18.5^{\prime}$ & 4720.3 & 7.5 & 275 & 51024 \\
\hline 10 & 7 & 1975 & 2110 & $39^{\circ} 14.3^{\prime}$ & $-53^{\circ} 20.1^{\prime}$ & 4721.5 & 4.7 & 275 & 51054 \\
\hline 10 & 7 & 1975 & 2206 & $39^{\circ} 14.7^{\prime}$ & $-53^{\circ} 25.8^{\prime}$ & 4726.0 & 8.5 & 88 & 51035 \\
\hline 10 & 7 & 1975 & 2228 & $39^{\circ} 14.8^{\prime}$ & $-53^{\circ} 21.8^{\prime}$ & 4729.1 & 0.0 & 90 & 51035 \\
\hline 12 & 7 & 1975 & 1015 & $39^{\circ} 14.8^{\prime}$ & $-53^{\circ} 21.8^{\prime}$ & 4729.1 & 6.6 & 51 & 51035 \\
\hline 12 & 7 & 1975 & 1035 & $39^{\circ} 16.2^{\prime}$ & $-53^{\circ} 19.6^{\prime}$ & 4731.3 & 9.5 & 51 & 51032 \\
\hline 12 & 7 & 1975 & 1218 & $39^{\circ} 26.5^{\prime}$ & $-53^{\circ} 03.2^{\prime}$ & 4747.6 & 9.8 & 50 & 51030 \\
\hline 12 & 7 & 1975 & 1308 & $39^{\circ} 31.7^{\prime}$ & $-52^{\circ} 55.1^{\prime}$ & 4755.7 & 9.8 & 50 & 51030 \\
\hline 12 & 7 & 1975 & 1404 & $39^{\circ} 37.6^{\prime}$ & $-52^{\circ} 46.1^{\prime}$ & 4764.8 & 9.6 & 50 & 51028 \\
\hline 12 & 7 & 1975 & 1454 & $39^{\circ} 42.7^{\prime}$ & $-52^{\circ} 38.1^{\prime}$ & 4772.8 & 10.0 & 49 & 51028 \\
\hline 12 & 7 & 1975 & 1606 & $39^{\circ} 50.5^{\prime}$ & $-52^{\circ} 26.3^{\prime}$ & 4784.8 & 10.0 & 48 & 51030 \\
\hline 12 & 7 & 1975 & 1710 & $39^{\circ} 57.7^{\prime}$ & $-52^{\circ} 16.0^{\prime}$ & 4795.5 & 9.8 & 46 & 51033 \\
\hline 12 & 7 & 1975 & 1754 & $40^{\circ} 02.7^{\prime}$ & $-52^{\circ} 09.2^{\prime}$ & 4802.7 & 10.3 & 47 & 51037 \\
\hline 12 & 7 & 1975 & 1856 & $40^{\circ} 10.0^{\prime}$ & $-51^{\circ} 59.1^{\prime}$ & 4813.3 & 12.1 & 48 & 51037 \\
\hline 12 & 7 & 1975 & 1928 & $40^{\circ} 14.3^{\prime}$ & $-51^{\circ} 52.8^{\prime}$ & 4819.8 & 12.1 & 52 & 51031 \\
\hline 12 & 7 & 1975 & 2015 & $40^{\circ} 20.1^{\prime}$ & $-51^{\circ} 43.0^{\prime}$ & 4829.2 & 7.3 & 237 & 51032 \\
\hline 12 & 7 & 1975 & 2021 & $40^{\circ} 19.7^{\prime}$ & $-51^{\circ} 43.8^{\prime}$ & 4830.0 & 4.3 & 244 & 51036 \\
\hline 12 & 7 & 1975 & 2040 & $40^{\circ} 19.1^{\prime}$ & $-51^{\circ} 45.4^{\prime}$ & 4831.3 & 4.7 & 246 & 51044 \\
\hline 12 & 7 & 1975 & 2115 & $40^{\circ} 18.0^{\prime}$ & $-51^{\circ} 48.7^{\prime}$ & 4834.1 & 8.9 & 48 & 51043 \\
\hline 12 & 7 & 1975 & 2212 & $40^{\circ} 23.6^{\prime}$ & $-51^{\circ} 40.4^{\prime}$ & 4842.5 & 4.9 & 167 & 51027 \\
\hline 12 & 7 & 1975 & 2237 & $40^{\circ} 21.6^{\prime}$ & $-51^{\circ} 39.8^{\prime}$ & 4844.6 & 0.0 & 0 & 51027 \\
\hline 15 & 7 & 1975 & 658 & $40^{\circ} 21.7^{\prime}$ & $-51^{\circ} 39.8^{\prime}$ & 4844.7 & 2.9 & 249 & 51030 \\
\hline 15 & 7 & 1975 & 715 & $40^{\circ} 21.4^{\prime}$ & $-51^{\circ} 40.8^{\prime}$ & 4845.5 & 7.4 & 262 & 51079 \\
\hline 15 & 7 & 1975 & 834 & $40^{\circ} 20.0^{\prime}$ & $-51^{\circ} 53.4^{\prime}$ & 4855.2 & 6.7 & 263 & 51142 \\
\hline 15 & 7 & 1975 & 1020 & $40^{\circ} 18.6^{\prime}$ & $-52^{\circ} 08.9^{\prime}$ & 4867.1 & 8.4 & 260 & 51156 \\
\hline 15 & 7 & 1975 & 1040 & $40^{\circ} 18.1^{\prime}$ & $-52^{\circ} 12.5^{\prime}$ & 4869.9 & 8.3 & 249 & 51182 \\
\hline 15 & 7 & 1975 & 1140 & $40^{\circ} 15.1^{\prime}$ & $-52^{\circ} 22.6^{\prime}$ & 4878.2 & 10.1 & 248 & 51208 \\
\hline 15 & 7 & 1975 & 1230 & $40^{\circ} 11.9^{\prime}$ & $-52^{\circ} 32.8^{\prime}$ & 4886.6 & 8.8 & 64 & 51203 \\
\hline 15 & 7 & 1975 & 1244 & $40^{\circ} 12.8^{\prime}$ & $-52^{\circ} 30.4^{\prime}$ & 4888.6 & 6.8 & 64 & 51201 \\
\hline 15 & 7 & 1975 & 1250 & $40^{\circ} 13.1^{\prime}$ & $-52^{\circ} 29.6^{\prime}$ & 4889.3 & 4.3 & 62 & 51200 \\
\hline 15 & 7 & 1975 & 1256 & $40^{\circ} 13.3^{\prime}$ & $-52^{\circ} 29.1^{\prime}$ & 4889.7 & 10.1 & 248 & 51216 \\
\hline 15 & 7 & 1975 & 1326 & $40^{\circ} 11.4^{\prime}$ & $-52^{\circ} 35.2^{\prime}$ & 4894.8 & 9.2 & 244 & 51255 \\
\hline 15 & 7 & 1975 & 1510 & $40^{\circ} 04.4^{\prime}$ & $-52^{\circ} 54.0^{\prime}$ & 4910.7 & 9.1 & 246 & 51285 \\
\hline 15 & 7 & 1975 & 1620 & $40^{\circ} 00.1^{\prime}$ & $-53^{\circ} 06.7^{\prime}$ & 4921.4 & 9.1 & 253 & 51307 \\
\hline 15 & 7 & 1975 & 1658 & $39^{\circ} 58.4^{\prime}$ & $-53^{\circ} 13.9^{\prime}$ & 4927.1 & 9.2 & 253 & 51347 \\
\hline 15 & 7 & 1975 & 1808 & $39^{\circ} 55.2^{\prime}$ & $-53^{\circ} 27.2^{\prime}$ & 4937.8 & 9.9 & 252 & 51379 \\
\hline 15 & 7 & 1975 & 1900 & $39^{\circ} 52.6^{\prime}$ & $-53^{\circ} 37.9^{\prime}$ & 4946.4 & 9.7 & 254 & 51406 \\
\hline 15 & 7 & 1975 & 1942 & $39^{\circ} 50.7^{\prime}$ & $-53^{\circ} 46.4^{\prime}$ & 4953.2 & 9.3 & 252 & 51527 \\
\hline 15 & 7 & 1975 & 2316 & $39^{\circ} 40.6^{\prime}$ & $-54^{\circ} 27.3^{\prime}$ & 4986.3 & 9.5 & 252 & 51539 \\
\hline 15 & 7 & 1975 & 2338 & $39^{\circ} 39.5^{\prime}$ & $-54^{\circ} 31.6^{\prime}$ & 4989.7 & 9.0 & 252 & 51552 \\
\hline 16 & 7 & 1975 & 000 & $39^{\circ} 38.5^{\prime}$ & $-54^{\circ} 35.7^{\prime}$ & 4993.1 & 9.2 & 252 & 51568 \\
\hline
\end{tabular}


TABLE 1 - Continued

\begin{tabular}{|c|c|c|c|c|c|c|c|c|c|}
\hline Day & Mon & Year & Time $^{\mathrm{a}}$ & Latitude & Longitude & Distance (n. mi.) & Speed $(k t)$ & Course & Regional Mag \\
\hline $\begin{array}{l}16 \\
16 \\
16 \\
16 \\
16\end{array}$ & $\begin{array}{l}7 \\
7 \\
7 \\
7 \\
7\end{array}$ & $\begin{array}{l}1975 \\
1975 \\
1975 \\
1975 \\
1975\end{array}$ & $\begin{array}{l}030 \\
124 \\
424 \\
534 \\
606\end{array}$ & $\begin{array}{l}39^{\circ} 37.1^{\prime} \\
39^{\circ} 34.7^{\prime} \\
39^{\circ} 24.5^{\prime} \\
39^{\circ} 19.1^{\prime} \\
39^{\circ} 17.1^{\prime}\end{array}$ & $\begin{array}{l}-54^{\circ} 41.4^{\prime} \\
-54^{\circ} 51.4^{\prime} \\
-55^{\circ} 23.5^{\prime} \\
-55^{\circ} 35.0^{\prime} \\
-55^{\circ} 40.4^{\prime}\end{array}$ & $\begin{array}{l}4997.7 \\
5005.7 \\
5032.5 \\
5042.9 \\
5047.6\end{array}$ & $\begin{array}{l}9.0 \\
8.9 \\
8.9 \\
8.7 \\
8.5\end{array}$ & $\begin{array}{l}253 \\
248 \\
239 \\
244 \\
236\end{array}$ & $\begin{array}{l}51598 \\
51677 \\
51693 \\
51704 \\
51706\end{array}$ \\
\hline $\begin{array}{l}16 \\
16 \\
16 \\
16 \\
16\end{array}$ & $\begin{array}{l}7 \\
7 \\
7 \\
7 \\
7\end{array}$ & $\begin{array}{l}1975 \\
1975 \\
1975 \\
1975 \\
1975\end{array}$ & $\begin{array}{r}625 \\
702 \\
742 \\
1114 \\
1238\end{array}$ & $\begin{array}{l}39^{\circ} 15.6^{\prime} \\
39^{\circ} 13.1^{\prime} \\
39^{\circ} 10.7^{\prime} \\
39^{\circ} 01.5^{\prime} \\
38^{\circ} 58.8^{\prime}\end{array}$ & $\begin{array}{l}-55^{\circ} 43.3^{\prime} \\
-55^{\circ} 49.2^{\prime} \\
-55^{\circ} 55.9^{\prime} \\
-56^{\circ} 32.2^{\prime} \\
-56^{\circ} 46.6^{\prime}\end{array}$ & $\begin{array}{l}5050.3 \\
5055.5 \\
5061.2 \\
5090.8 \\
5102.3\end{array}$ & $\begin{array}{l}8.4 \\
8.6 \\
8.4 \\
8.2 \\
8.4\end{array}$ & $\begin{array}{l}241 \\
245 \\
252 \\
256 \\
258\end{array}$ & $\begin{array}{l}51716 \\
51730 \\
51834 \\
51882 \\
51911\end{array}$ \\
\hline $\begin{array}{l}16 \\
16 \\
16 \\
17 \\
17\end{array}$ & $\begin{array}{l}7 \\
7 \\
7 \\
7 \\
7\end{array}$ & $\begin{array}{l}1975 \\
1975 \\
1975 \\
1975 \\
1975\end{array}$ & $\begin{array}{r}1326 \\
1426 \\
1512 \\
000 \\
128\end{array}$ & $\begin{array}{l}38^{\circ} 57.4^{\prime} \\
38^{\circ} 55.7^{\prime} \\
38^{\circ} 54.6^{\prime} \\
38^{\circ} 43.5^{\prime} \\
38^{\circ} 44.8^{\prime}\end{array}$ & $\begin{array}{l}-56^{\circ} 55.0^{\prime} \\
-57^{\circ} 05.4^{\prime} \\
-57^{\circ} 13.6^{\prime} \\
-58^{\circ} 44.6^{\prime} \\
-59^{\circ} 00.7^{\prime}\end{array}$ & $\begin{array}{l}5109.0 \\
5117.3 \\
5123.7 \\
5195.5 \\
5208.1\end{array}$ & $\begin{array}{l}8.3 \\
8.4 \\
8.2 \\
8.6 \\
7.1\end{array}$ & $\begin{array}{l}258 \\
260 \\
261 \\
276 \\
266\end{array}$ & $\begin{array}{l}51947 \\
51977 \\
52316 \\
52399 \\
52416\end{array}$ \\
\hline $\begin{array}{l}17 \\
17 \\
17 \\
17 \\
17\end{array}$ & $\begin{array}{l}7 \\
7 \\
7 \\
7 \\
7\end{array}$ & $\begin{array}{l}1975 \\
1975 \\
1975 \\
1975 \\
1975\end{array}$ & $\begin{array}{l}155 \\
224 \\
254 \\
424 \\
440\end{array}$ & $\begin{array}{l}38^{\circ} 44.6^{\prime} \\
38^{\circ} 43.9^{\prime} \\
38^{\circ} 43.0^{\prime} \\
38^{\circ} 40.3^{\prime} \\
38^{\circ} 39.8^{\prime}\end{array}$ & $\begin{array}{l}-59^{\circ} 04.8^{\prime} \\
-59^{\circ} 09.1^{\prime} \\
-59^{\circ} 14.2^{\prime} \\
-59^{\circ} 29.3^{\prime} \\
-59^{\circ} 32.5^{\prime}\end{array}$ & $\begin{array}{l}5211.3 \\
5214.8 \\
5218.8 \\
5230.9 \\
5233.5\end{array}$ & $\begin{array}{l}7.1 \\
8.2 \\
8.1 \\
9.6 \\
6.7\end{array}$ & $\begin{array}{l}258 \\
257 \\
257 \\
259 \\
262\end{array}$ & $\begin{array}{l}52431 \\
52448 \\
52497 \\
52507 \\
52527\end{array}$ \\
\hline $\begin{array}{l}17 \\
17 \\
17 \\
17 \\
17\end{array}$ & $\begin{array}{l}7 \\
7 \\
7 \\
7 \\
7\end{array}$ & $\begin{array}{l}1975 \\
1975 \\
1975 \\
1975 \\
1975\end{array}$ & $\begin{array}{r}518 \\
610 \\
632 \\
834 \\
1016\end{array}$ & $\begin{array}{l}38^{\circ} 39.2^{\prime} \\
38^{\circ} 38.3^{\prime} \\
38^{\circ} 38.1^{\prime} \\
38^{\circ} 35.4^{\prime} \\
38^{\circ} 35.2^{\prime}\end{array}$ & $\begin{array}{l}-59^{\circ} 37.9^{\prime} \\
-59^{\circ} 45.0^{\prime} \\
-59^{\circ} 48.2^{\prime} \\
-60^{\circ} 05.2^{\prime} \\
-60^{\circ} 18.3^{\prime}\end{array}$ & $\begin{array}{l}5237.7 \\
5243.4 \\
5245.9 \\
5259.4 \\
5269.7\end{array}$ & $\begin{array}{l}6.5 \\
6.8 \\
6.7 \\
6.0 \\
4.7\end{array}$ & $\begin{array}{l}261 \\
265 \\
259 \\
269 \\
265\end{array}$ & $\begin{array}{l}52553 \\
52566 \\
52623 \\
52680 \\
52692\end{array}$ \\
\hline $\begin{array}{l}17 \\
17 \\
17 \\
17 \\
17\end{array}$ & $\begin{array}{l}7 \\
7 \\
7 \\
7 \\
7\end{array}$ & $\begin{array}{l}1975 \\
1975 \\
1975 \\
1975 \\
1975\end{array}$ & $\begin{array}{l}1045 \\
1150 \\
1336 \\
1422 \\
1446\end{array}$ & $\begin{array}{l}38^{\circ} 35.0^{\prime} \\
38^{\circ} 31.8^{\prime} \\
38^{\circ} 27.9^{\prime} \\
38^{\circ} 25.5^{\prime} \\
38^{\circ} 24.5^{\prime}\end{array}$ & $\begin{array}{l}-60^{\circ} 21.2^{\prime} \\
-60^{\circ} 25.4^{\prime} \\
-60^{\circ} 34.8^{\prime} \\
-60^{\circ} 38.2^{\prime} \\
-60^{\circ} 40.1^{\prime}\end{array}$ & $\begin{array}{l}5271.9 \\
5276.5 \\
5284.8 \\
5288.4 \\
5290.2\end{array}$ & $\begin{array}{l}4.2 \\
4.7 \\
4.7 \\
4.5 \\
4.6\end{array}$ & $\begin{array}{l}226 \\
242 \\
228 \\
236 \\
235\end{array}$ & $\begin{array}{l}52687 \\
52701 \\
52698 \\
52699 \\
52702\end{array}$ \\
\hline $\begin{array}{l}17 \\
17 \\
17 \\
17 \\
17\end{array}$ & $\begin{array}{l}7 \\
7 \\
7 \\
7 \\
7\end{array}$ & $\begin{array}{l}1975 \\
1975 \\
1975 \\
1975 \\
1975\end{array}$ & $\begin{array}{l}1610 \\
1630 \\
1724 \\
1910 \\
1930\end{array}$ & $\begin{array}{l}38^{\circ} 20.8^{\prime} \\
38^{\circ} 19.9^{\prime} \\
38^{\circ} 14.9^{\prime} \\
38^{\circ} 04.5^{\prime} \\
38^{\circ} 02.3^{\prime}\end{array}$ & $\begin{array}{l}-60^{\circ} 46.9^{\prime} \\
-60^{\circ} 49.0^{\prime} \\
-60^{\circ} 42.3^{\prime} \\
-60^{\circ} 27.8^{\prime} \\
-60^{\circ} 26.3^{\prime}\end{array}$ & $\begin{array}{l}5296.7 \\
5298.6 \\
5305.8 \\
5321.3 \\
5323.8\end{array}$ & $\begin{array}{l}5.6 \\
8.1 \\
8.7 \\
7.5 \\
7.3 \\
\end{array}$ & $\begin{array}{l}241 \\
134 \\
132 \\
152 \\
173\end{array}$ & $\begin{array}{l}52705 \\
52639 \\
52498 \\
52475 \\
52465\end{array}$ \\
\hline $\begin{array}{l}17 \\
17 \\
17 \\
17 \\
17\end{array}$ & $\begin{array}{l}7 \\
7 \\
7 \\
7 \\
7\end{array}$ & $\begin{array}{l}1975 \\
1975 \\
1975 \\
1975 \\
1975\end{array}$ & $\begin{array}{l}1940 \\
2126 \\
2200 \\
2312 \\
2335\end{array}$ & $\begin{array}{l}38^{\circ} 01.1^{\prime} \\
37^{\circ} 47.1^{\prime} \\
37^{\circ} 41.9^{\prime} \\
37^{\circ} 31.8^{\prime} \\
37^{\circ} 28.9^{\prime}\end{array}$ & $\begin{array}{l}-60^{\circ} 26.1^{\prime} \\
-60^{\circ} 22.9^{\prime} \\
-60^{\circ} 21.5^{\prime} \\
-60^{\circ} 13.6^{\prime} \\
-60^{\circ} 12.3^{\prime}\end{array}$ & $\begin{array}{l}5325.0 \\
5339.2 \\
5344.5 \\
5356.4 \\
5359.5\end{array}$ & $\begin{array}{l}8.1 \\
9.4 \\
9.9 \\
8.0 \\
8.4\end{array}$ & $\begin{array}{l}170 \\
168 \\
148 \\
160 \\
172\end{array}$ & $\begin{array}{l}52346 \\
52301 \\
52189 \\
52162 \\
52147\end{array}$ \\
\hline $\begin{array}{l}17 \\
18 \\
18 \\
18 \\
18\end{array}$ & $\begin{array}{l}7 \\
7 \\
7 \\
7 \\
7\end{array}$ & $\begin{array}{l}1975 \\
1975 \\
1975 \\
1975 \\
1975\end{array}$ & $\begin{array}{r}2348 \\
000 \\
134 \\
204 \\
222\end{array}$ & $\begin{array}{l}37^{\circ} 27.1^{\prime} \\
37^{\circ} 25.3^{\prime} \\
37^{\circ} 11.6^{\prime} \\
37^{\circ} 07.0^{\prime} \\
37^{\circ} 05.1^{\prime}\end{array}$ & $\begin{array}{l}-60^{\circ} 12.0^{\prime} \\
-60^{\circ} 11.7^{\prime} \\
-60^{\circ} 09.3^{\prime} \\
-60^{\circ} 08.8^{\prime} \\
-60^{\circ} 06.7^{\prime}\end{array}$ & $\begin{array}{l}5361.3 \\
5363.1 \\
5376.9 \\
5381.6 \\
5384.1\end{array}$ & $\begin{array}{l}9.1 \\
8.8 \\
9.2 \\
8.4 \\
7.6\end{array}$ & $\begin{array}{l}172 \\
172 \\
175 \\
139 \\
130\end{array}$ & $\begin{array}{l}52132 \\
52016 \\
51979 \\
51955 \\
51885\end{array}$ \\
\hline $\begin{array}{l}18 \\
18 \\
18 \\
18 \\
18\end{array}$ & $\begin{array}{l}7 \\
7 \\
7 \\
7 \\
7\end{array}$ & $\begin{array}{l}1975 \\
1975 \\
1975 \\
1975 \\
1975\end{array}$ & $\begin{array}{l}322 \\
330 \\
332 \\
340 \\
410\end{array}$ & $\begin{array}{l}37^{\circ} 00.2^{\prime} \\
36^{\circ} 59.3^{\prime} \\
36^{\circ} 59.6^{\prime} \\
37^{\circ} 00.2^{\prime} \\
37^{\circ} 03.8^{\prime}\end{array}$ & $\begin{array}{l}-59^{\circ} 59.5^{\prime} \\
-60^{\circ} 00.7^{\prime} \\
-60^{\circ} 01.0^{\prime} \\
-60^{\circ} 02.6^{\prime} \\
-60^{\circ} 05.3^{\prime}\end{array}$ & $\begin{array}{l}5391.6 \\
5393.0 \\
5393.3 \\
5394.8 \\
5399.0\end{array}$ & $\begin{array}{r}9.8 \\
11.6 \\
10.6 \\
8.4 \\
10.0\end{array}$ & $\begin{array}{l}227 \\
321 \\
295 \\
329 \\
332\end{array}$ & $\begin{array}{l}51883 \\
51887 \\
51899 \\
51939 \\
52045\end{array}$ \\
\hline $\begin{array}{l}18 \\
18 \\
18 \\
18 \\
20\end{array}$ & $\begin{array}{l}7 \\
7 \\
7 \\
7 \\
7\end{array}$ & $\begin{array}{l}1975 \\
1975 \\
1975 \\
1975 \\
1975\end{array}$ & $\begin{array}{r}518 \\
538 \\
617 \\
620 \\
2310\end{array}$ & $\begin{array}{l}37^{\circ} 13.8^{\prime} \\
37^{\circ} 16.8^{\prime} \\
37^{\circ} 21.9^{\prime} \\
37^{\circ} 22.2^{\prime} \\
37^{\circ} 21.4^{\prime}\end{array}$ & $\begin{array}{l}-60^{\circ} 11.9^{\prime} \\
-60^{\circ} 12.7^{\prime} \\
-60^{\circ} 09.6^{\prime} \\
-60^{\circ} 09.4^{\prime} \\
-60^{\circ} 12.5^{\prime}\end{array}$ & $\begin{array}{l}5410.3 \\
5413.3 \\
5419.0 \\
5419.3 \\
5421.9\end{array}$ & $\begin{array}{l}9.2 \\
8.7 \\
6.8 \\
0.0 \\
7.6\end{array}$ & $\begin{array}{r}348 \\
26 \\
28 \\
252 \\
207\end{array}$ & $\begin{array}{l}52071 \\
52096 \\
52098 \\
52105 \\
52097\end{array}$ \\
\hline $\begin{array}{l}20 \\
21 \\
21 \\
21 \\
21\end{array}$ & $\begin{array}{l}7 \\
7 \\
7 \\
7 \\
7\end{array}$ & $\begin{array}{l}1975 \\
1975 \\
1975 \\
1975 \\
1975\end{array}$ & $\begin{array}{r}2325 \\
000 \\
250 \\
346 \\
442\end{array}$ & $\begin{array}{l}37^{\circ} 19.7^{\prime} \\
37^{\circ} 14.2^{\prime} \\
36^{\circ} 47.7^{\prime} \\
36^{\circ} 39.2^{\prime} \\
36^{\circ} 31.2^{\prime}\end{array}$ & $\begin{array}{l}-60^{\circ} 13.6^{\prime} \\
-60^{\circ} 17.0^{\prime} \\
-60^{\circ} 33.7^{\prime} \\
-60^{\circ} 38.6^{\prime} \\
-60^{\circ} 42.6^{\prime}\end{array}$ & $\begin{array}{l}5423.8 \\
5430.0 \\
5459.6 \\
5469.0 \\
5477.6\end{array}$ & $\begin{array}{r}10.5 \\
10.5 \\
10.0 \\
9.2 \\
9.2\end{array}$ & $\begin{array}{l}206 \\
207 \\
205 \\
202 \\
207\end{array}$ & $\begin{array}{l}52071 \\
51941 \\
51897 \\
51852 \\
51826\end{array}$ \\
\hline
\end{tabular}


TABLE 1 - Continued

\begin{tabular}{|c|c|c|c|c|c|c|c|c|c|}
\hline Day & Mon & Year & $\operatorname{Time}^{\mathrm{a}}$ & Latitude & Longitude & Distance (n. mi.) & Speed $(k t)$ & Course & Regional Mag \\
\hline $\begin{array}{l}21 \\
21 \\
21 \\
21 \\
21\end{array}$ & $\begin{array}{l}7 \\
7 \\
7 \\
7 \\
7\end{array}$ & $\begin{array}{l}1975 \\
1975 \\
1975 \\
1975 \\
1975\end{array}$ & $\begin{array}{r}520 \\
630 \\
708 \\
822 \\
1008\end{array}$ & $\begin{array}{l}36^{\circ} 26.0^{\prime} \\
36^{\circ} 17.0^{\prime} \\
36^{\circ} 12.0^{\prime} \\
36^{\circ} 01.6^{\prime} \\
35^{\circ} 47.7^{\prime}\end{array}$ & $\begin{array}{l}-60^{\circ} 45.9^{\prime} \\
-60^{\circ} 51.3^{\prime} \\
-60^{\circ} 54.5^{\prime} \\
-61^{\circ} 00.5^{\prime} \\
-61^{\circ} 09.5^{\prime}\end{array}$ & $\begin{array}{l}5483.4 \\
5493.4 \\
5499.1 \\
5510.5 \\
5526.2\end{array}$ & $\begin{array}{l}8.6 \\
8.9 \\
9.3 \\
8.9 \\
8.9\end{array}$ & $\begin{array}{l}206 \\
207 \\
205 \\
208 \\
210\end{array}$ & $\begin{array}{l}51779 \\
51753 \\
51697 \\
51626 \\
51547\end{array}$ \\
\hline $\begin{array}{l}21 \\
21 \\
21 \\
21 \\
21\end{array}$ & $\begin{array}{l}7 \\
7 \\
7 \\
7 \\
7\end{array}$ & $\begin{array}{l}1975 \\
1975 \\
1975 \\
1975 \\
1975\end{array}$ & $\begin{array}{l}1210 \\
1254 \\
1530 \\
1716 \\
1742\end{array}$ & $\begin{array}{l}35^{\circ} 31.9^{\prime} \\
35^{\circ} 26.3^{\prime} \\
35^{\circ} 07.6^{\prime} \\
34^{\circ} 54.9^{\prime} \\
34^{\circ} 51.9^{\prime}\end{array}$ & $\begin{array}{l}-61^{\circ} 20.6^{\prime} \\
-61^{\circ} 24.1^{\prime} \\
-61^{\circ} 37.3^{\prime} \\
-61^{\circ} 46.8^{\prime} \\
-61^{\circ} 49.1^{\prime}\end{array}$ & $\begin{array}{l}5544.4 \\
5550.7 \\
5572.3 \\
5587.2 \\
5590.7\end{array}$ & $\begin{array}{l}8.6 \\
8.3 \\
8.4 \\
8.2 \\
8.3\end{array}$ & $\begin{array}{l}207 \\
210 \\
211 \\
212 \\
213\end{array}$ & $\begin{array}{l}51517 \\
51421 \\
51358 \\
51343 \\
51322\end{array}$ \\
\hline $\begin{array}{l}21 \\
21 \\
21 \\
21 \\
21\end{array}$ & $\begin{array}{l}7 \\
7 \\
7 \\
7 \\
7\end{array}$ & $\begin{array}{l}1975 \\
1975 \\
1975 \\
1975 \\
1975\end{array}$ & $\begin{array}{l}1820 \\
2008 \\
2118 \\
2300 \\
2306\end{array}$ & $\begin{array}{l}34^{\circ} 47.5^{\prime} \\
34^{\circ} 35.1^{\prime} \\
34^{\circ} 26.8^{\prime} \\
34^{\circ} 14.4^{\prime} \\
34^{\circ} 13.8^{\prime}\end{array}$ & $\begin{array}{l}-61^{\circ} 52.6^{\prime} \\
-62^{\circ} 01.4^{\prime} \\
-62^{\circ} 08.3^{\prime} \\
-62^{\circ} 18.1^{\prime} \\
-62^{\circ} 18.9^{\prime}\end{array}$ & $\begin{array}{l}5596.0 \\
5610.3 \\
5620.4 \\
5635.2 \\
5636.1\end{array}$ & $\begin{array}{l}8.0 \\
8.6 \\
8.7 \\
9.0 \\
9.4\end{array}$ & $\begin{array}{l}210 \\
214 \\
213 \\
228 \\
233\end{array}$ & $\begin{array}{l}51256 \\
51216 \\
51154 \\
51152 \\
51143\end{array}$ \\
\hline $\begin{array}{l}22 \\
22 \\
22 \\
22 \\
22\end{array}$ & $\begin{array}{l}7 \\
7 \\
7 \\
7 \\
7\end{array}$ & $\begin{array}{l}1975 \\
1975 \\
1975 \\
1975 \\
1975\end{array}$ & $\begin{array}{l}000 \\
006 \\
056 \\
152 \\
220\end{array}$ & $\begin{array}{l}34^{\circ} 08.7^{\prime} \\
34^{\circ} 08.1^{\prime} \\
34^{\circ} 03.0^{\prime} \\
33^{\circ} 57.4^{\prime} \\
33^{\circ} 54.5^{\prime}\end{array}$ & $\begin{array}{l}-62^{\circ} 27.0^{\prime} \\
-62^{\circ} 27.9^{\prime} \\
-62^{\circ} 35.3^{\prime} \\
-62^{\circ} 44.6^{\prime} \\
-62^{\circ} 48.8^{\prime}\end{array}$ & $\begin{array}{l}5644.5 \\
5645.5 \\
5653.4 \\
5663.0 \\
5667.5\end{array}$ & $\begin{array}{r}9.6 \\
9.6 \\
10.2 \\
9.7 \\
9.5\end{array}$ & $\begin{array}{l}231 \\
230 \\
234 \\
230 \\
225\end{array}$ & $\begin{array}{l}51142 \\
51130 \\
51122 \\
51115 \\
51078\end{array}$ \\
\hline $\begin{array}{l}22 \\
22 \\
22 \\
22 \\
22\end{array}$ & $\begin{array}{l}7 \\
7 \\
7 \\
7 \\
7\end{array}$ & $\begin{array}{l}1975 \\
1975 \\
1975 \\
1975 \\
1975\end{array}$ & $\begin{array}{l}354 \\
422 \\
538 \\
804 \\
912\end{array}$ & $\begin{array}{l}33^{\circ} 44.0^{\prime} \\
33^{\circ} 40.5^{\prime} \\
33^{\circ} 31.1^{\prime} \\
33^{\circ} 12.7^{\prime} \\
33^{\circ} 04.7^{\prime}\end{array}$ & $\begin{array}{l}-63^{\circ} 01.5^{\prime} \\
-63^{\circ} 04.5^{\prime} \\
-63^{\circ} 16.6^{\prime} \\
-63^{\circ} 31.3^{\prime} \\
-63^{\circ} 37.9^{\prime}\end{array}$ & $\begin{array}{l}5682.4 \\
5686.7 \\
5700.5 \\
5722.6 \\
5732.3\end{array}$ & $\begin{array}{r}9.2 \\
10.9 \\
9.1 \\
8.6 \\
9.1\end{array}$ & $\begin{array}{l}215 \\
227 \\
214 \\
215 \\
219\end{array}$ & $\begin{array}{l}51061 \\
51030 \\
50931 \\
50888 \\
50869\end{array}$ \\
\hline $\begin{array}{l}22 \\
22 \\
22 \\
22 \\
22\end{array}$ & $\begin{array}{l}7 \\
7 \\
7 \\
7 \\
7\end{array}$ & $\begin{array}{l}1975 \\
1975 \\
1975 \\
1975 \\
1975\end{array}$ & $\begin{array}{r}945 \\
1100 \\
1122 \\
1308 \\
1352\end{array}$ & $\begin{array}{l}33^{\circ} 00.8^{\prime} \\
32^{\circ} 53.3^{\prime} \\
32^{\circ} 51.1^{\prime} \\
32^{\circ} 41.1^{\prime} \\
32^{\circ} 37.0^{\prime}\end{array}$ & $\begin{array}{l}-63^{\circ} 41.7^{\prime} \\
-63^{\circ} 52.1^{\prime} \\
-63^{\circ} 55.1^{\prime} \\
-64^{\circ} 08.6^{\prime} \\
-64^{\circ} 15.1^{\prime}\end{array}$ & $\begin{array}{l}5737.3 \\
5748.9 \\
5752.2 \\
5767.3 \\
5774.2\end{array}$ & $\begin{array}{l}9.2 \\
9.1 \\
8.6 \\
9.3 \\
8.1\end{array}$ & $\begin{array}{l}229 \\
229 \\
229 \\
233 \\
227\end{array}$ & $\begin{array}{l}50845 \\
50838 \\
50804 \\
50794 \\
50782\end{array}$ \\
\hline $\begin{array}{l}22 \\
24 \\
24 \\
24 \\
24\end{array}$ & $\begin{array}{l}7 \\
7 \\
7 \\
7 \\
7\end{array}$ & $\begin{array}{l}1975 \\
1975 \\
1975 \\
1975 \\
1975\end{array}$ & $\begin{array}{l}1426 \\
1155 \\
1210 \\
1318 \\
1540\end{array}$ & $\begin{array}{l}32^{\circ} 33.9^{\prime} \\
32^{\circ} 24.8^{\prime} \\
32^{\circ} 23.1^{\prime} \\
32^{\circ} 12.2^{\prime} \\
31^{\circ} 55.0^{\prime}\end{array}$ & $\begin{array}{l}-64^{\circ} 19.1^{\prime} \\
-64^{\circ} 34.8^{\prime} \\
-64^{\circ} 34.3^{\prime} \\
-64^{\circ} 31.2^{\prime} \\
-64^{\circ} 25.3^{\prime}\end{array}$ & $\begin{array}{l}5778.7 \\
5794.8 \\
5796.6 \\
5807.8 \\
5825.7\end{array}$ & $\begin{array}{l}0.4 \\
7.0 \\
9.9 \\
7.6 \\
7.5\end{array}$ & $\begin{array}{l}236 \\
166 \\
166 \\
164 \\
170\end{array}$ & $\begin{array}{l}50763 \\
50746 \\
50636 \\
50457 \\
50355\end{array}$ \\
\hline $\begin{array}{l}24 \\
24 \\
24 \\
24 \\
24\end{array}$ & $\begin{array}{l}7 \\
7 \\
7 \\
7 \\
7\end{array}$ & $\begin{array}{l}1975 \\
1975 \\
1975 \\
1975 \\
1975\end{array}$ & $\begin{array}{l}1704 \\
1726 \\
1852 \\
1920 \\
2022\end{array}$ & $\begin{array}{l}31^{\circ} 44.7^{\prime} \\
31^{\circ} 42.0^{\prime} \\
31^{\circ} 30.6^{\prime} \\
31^{\circ} 26.9^{\prime} \\
31^{\circ} 18.9^{\prime}\end{array}$ & $\begin{array}{l}-64^{\circ} 23.2^{\prime} \\
-64^{\circ} 22.5^{\prime} \\
-64^{\circ} 19.7^{\prime} \\
-64^{\circ} 19.0^{\prime} \\
-64^{\circ} 18.0^{\prime}\end{array}$ & $\begin{array}{l}5836.1 \\
5838.9 \\
5850.5 \\
5854.3 \\
5862.3\end{array}$ & $\begin{array}{l}7.5 \\
8.1 \\
8.0 \\
7.8 \\
9.6\end{array}$ & $\begin{array}{l}168 \\
168 \\
171 \\
174 \\
167\end{array}$ & $\begin{array}{l}50328 \\
50213 \\
50176 \\
50099 \\
50062\end{array}$ \\
\hline $\begin{array}{l}24 \\
24 \\
24 \\
24 \\
31\end{array}$ & $\begin{array}{l}7 \\
7 \\
7 \\
7 \\
7\end{array}$ & $\begin{array}{l}1975 \\
1975 \\
1975 \\
1975 \\
1975\end{array}$ & $\begin{array}{l}2045 \\
2122 \\
2135 \\
2202 \\
2053\end{array}$ & $\begin{array}{l}31^{\circ} 15.3^{\prime} \\
31^{\circ} 09.8^{\prime} \\
31^{\circ} 08.5^{\prime} \\
31^{\circ} 11.2^{\prime} \\
31^{\circ} 11.2^{\prime}\end{array}$ & $\begin{array}{l}-64^{\circ} 17.0^{\prime} \\
-64^{\circ} 14.1^{\prime} \\
-64^{\circ} 13.4^{\prime} \\
-64^{\circ} 14.9^{\prime} \\
-64^{\circ} 14.9^{\prime}\end{array}$ & $\begin{array}{l}5866.0 \\
5872.1 \\
5873.5 \\
5876.5 \\
5876.5\end{array}$ & $\begin{array}{l}9.8 \\
6.6 \\
6.6 \\
0.0 \\
9.0\end{array}$ & $\begin{array}{r}156 \\
155 \\
335 \\
90 \\
323\end{array}$ & $\begin{array}{l}50000 \\
49986 \\
50016 \\
50015 \\
50077\end{array}$ \\
\hline $\begin{array}{r}31 \\
31 \\
1 \\
1 \\
1\end{array}$ & $\begin{array}{l}7 \\
7 \\
8 \\
8 \\
8\end{array}$ & $\begin{array}{l}1975 \\
1975 \\
1975 \\
1975 \\
1975\end{array}$ & $\begin{array}{r}2134 \\
2250 \\
000 \\
040 \\
340\end{array}$ & $\begin{array}{l}31^{\circ} 16.1^{\prime} \\
31^{\circ} 21.7^{\prime} \\
31^{\circ} 26.4^{\prime} \\
31^{\circ} 29.0^{\prime} \\
31^{\circ} 36.5^{\prime}\end{array}$ & $\begin{array}{l}-64^{\circ} 19.2^{\prime} \\
-64^{\circ} 35.9^{\prime} \\
-64^{\circ} 51.6^{\prime} \\
-65^{\circ} 00.5^{\prime} \\
-65^{\circ} 33.0^{\prime}\end{array}$ & $\begin{array}{l}5882.6 \\
5897.9 \\
5912.1 \\
5920.2 \\
5948.9\end{array}$ & $\begin{array}{r}12.1 \\
12.2 \\
12.0 \\
9.6 \\
7.8\end{array}$ & $\begin{array}{l}291 \\
289 \\
289 \\
285 \\
280\end{array}$ & $\begin{array}{l}50194 \\
50299 \\
50357 \\
50550 \\
50681\end{array}$ \\
\hline $\begin{array}{l}1 \\
1 \\
1 \\
1 \\
1\end{array}$ & $\begin{array}{l}8 \\
8 \\
8 \\
8 \\
8\end{array}$ & $\begin{array}{l}1975 \\
1975 \\
1975 \\
1975 \\
1975\end{array}$ & $\begin{array}{r}630 \\
900 \\
1030 \\
1236 \\
1336\end{array}$ & $\begin{array}{l}31^{\circ} 40.3^{\prime} \\
31^{\circ} 42.0^{\prime} \\
31^{\circ} 48.6^{\prime} \\
32^{\circ} 00.4^{\prime} \\
32^{\circ} 04.1^{\prime}\end{array}$ & $\begin{array}{l}-65^{\circ} 58.5^{\prime} \\
-66^{\circ} 21.0^{\prime} \\
-66^{\circ} 36.1^{\prime} \\
-66^{\circ} 56.1^{\prime} \\
-67^{\circ} 05.5^{\prime}\end{array}$ & $\begin{array}{l}5970.9 \\
5990.1 \\
6004.6 \\
6025.2 \\
6034.0\end{array}$ & $\begin{array}{l}7.7 \\
9.6 \\
9.8 \\
8.8 \\
9.1\end{array}$ & $\begin{array}{l}275 \\
297 \\
305 \\
295 \\
297\end{array}$ & $\begin{array}{l}50780 \\
50896 \\
51077 \\
51145 \\
51360\end{array}$ \\
\hline $\begin{array}{l}1 \\
1 \\
1 \\
1 \\
7\end{array}$ & $\begin{array}{l}8 \\
8 \\
8 \\
8 \\
8\end{array}$ & $\begin{array}{l}1975 \\
1975 \\
1975 \\
1975 \\
1975\end{array}$ & $\begin{array}{l}1635 \\
1653 \\
1715 \\
1719 \\
1219\end{array}$ & $\begin{array}{l}32^{\circ} 16.6^{\prime} \\
32^{\circ} 17.5^{\prime} \\
32^{\circ} 19.0^{\prime} \\
32^{\circ} 19.2^{\prime} \\
32^{\circ} 19.2^{\prime}\end{array}$ & $\begin{array}{l}-67^{\circ} 34.1^{\prime} \\
-67^{\circ} 36.0^{\prime} \\
-67^{\circ} 39.6^{\prime} \\
-67^{\circ} 40.0^{\prime} \\
-67^{\circ} 40.0^{\prime}\end{array}$ & $\begin{array}{l}6061.3 \\
6063.1 \\
6066.5 \\
6066.9 \\
6066.9\end{array}$ & $\begin{array}{l}6.1 \\
9.2 \\
5.9 \\
0.0 \\
6.9\end{array}$ & $\begin{array}{r}299 \\
296 \\
301 \\
90 \\
303\end{array}$ & $\begin{array}{l}51375 \\
51402 \\
51405 \\
51404 \\
51412\end{array}$ \\
\hline
\end{tabular}


TABLE 1 - Continued

\begin{tabular}{|c|c|c|c|c|c|c|c|c|c|}
\hline Day & Mon & Year & Time $^{\mathrm{a}}$ & Latitude & Longitude & Distance (n. mi.) & Speed $(k t)$ & Course & Regional Mag \\
\hline 7 & 8 & 1975 & 1227 & $32^{\circ} 19.7^{\prime}$ & $-67^{\circ} 40.9^{\prime}$ & 6067.8 & 9.5 & 299 & 51517 \\
\hline 7 & 8 & 1975 & 1350 & $32^{\circ} 26.1^{\prime}$ & $-67^{\circ} 54.5^{\prime}$ & 6080.9 & 10.4 & 55 & 51557 \\
\hline 7 & 8 & 1975 & 1600 & $32^{\circ} 39.0^{\prime}$ & $-67^{\circ} 32.5^{\prime}$ & 6103.5 & 8.7 & 57 & 51558 \\
\hline 7 & 8 & 1975 & 1609 & $32^{\circ} 39.7^{\prime}$ & $-67^{\circ} 31.2^{\prime}$ & 6104.8 & 10.1 & 204 & 51440 \\
\hline 7 & 8 & 1975 & 1755 & $32^{\circ} 23.3^{\prime}$ & $-67^{\circ} 39.7^{\prime}$ & 6122.7 & 6.7 & 206 & 51425 \\
\hline 7 & 8 & 1975 & 1816 & $32^{\circ} 21.2^{\prime}$ & $-67^{\circ} 40.9^{\prime}$ & 6125.1 & 10.2 & 203 & 51409 \\
\hline 7 & 8 & 1975 & 1830 & $32^{\circ} 19.0^{\prime}$ & $-67^{\circ} 42.0^{\prime}$ & 6127.4 & 9.3 & 200 & 51286 \\
\hline 7 & 8 & 1975 & 2020 & $32^{\circ} 02.9^{\prime}$ & $-67^{\circ} 48.9^{\prime}$ & 6144.6 & 9.7 & 54 & 51341 \\
\hline 7 & 8 & 1975 & 2314 & $32^{\circ} 19.4^{\prime}$ & $-67^{\circ} 21.8^{\prime}$ & 6172.8 & 9.9 & 325 & 51416 \\
\hline 8 & 8 & 1975 & 000 & $32^{\circ} 25.6^{\prime}$ & $-67^{\circ} 27.0^{\prime}$ & 6180.4 & 9.3 & 233 & 51411 \\
\hline 8 & 8 & 1975 & 015 & $32^{\circ} 24.2^{\prime}$ & $-67^{\circ} 29.2^{\prime}$ & 6182.7 & 9.7 & 251 & 51414 \\
\hline 8 & 8 & 1975 & 030 & $32^{\circ} 23.4^{\prime}$ & $-67^{\circ} 31.9^{\prime}$ & 6185.2 & 9.4 & 232 & 51403 \\
\hline 8 & 8 & 1975 & 100 & $32^{\circ} 20.5^{\prime}$ & $-67^{\circ} 36.3^{\prime}$ & 6189.9 & 6.6 & 233 & 51382 \\
\hline 8 & 8 & 1975 & 225 & $32^{\circ} 14.8^{\prime}$ & $-67^{\circ} 45.1^{\prime}$ & 6199.2 & 9.5 & 232 & 51361 \\
\hline 8 & 8 & 1975 & 319 & $32^{\circ} 09.5^{\prime}$ & $-67^{\circ} 53.0^{\prime}$ & 6207.8 & 9.9 & 327 & 51412 \\
\hline 8 & 8 & 1975 & 350 & $32^{\circ} 13.8^{\prime}$ & $-67^{\circ} 56.3^{\prime}$ & 6212.9 & 9.7 & 55 & 51475 \\
\hline 8 & 8 & 1975 & 736 & $32^{\circ} 34.6^{\prime}$ & $-67^{\circ} 20.7^{\prime}$ & 6249.5 & 9.4 & 108 & 51352 \\
\hline 8 & 8 & 1975 & 930 & $32^{\circ} 29.0^{\prime}$ & $-67^{\circ} 00.5^{\prime}$ & 6267.4 & 8.4 & 110 & 51195 \\
\hline 8 & 8 & 1975 & 1208 & $32^{\circ} 21.5^{\prime}$ & $-66^{\circ} 36.0^{\prime}$ & 6289.4 & 8.1 & 107 & 51181 \\
\hline 8 & 8 & 1975 & 1223 & $32^{\circ} 20.9^{\prime}$ & $-66^{\circ} 33.7^{\prime}$ & 6291.4 & 6.8 & 106 & 51038 \\
\hline 8 & 8 & 1975 & 1530 & $32^{\circ} 15.0^{\prime}$ & $-66^{\circ} 09.5^{\prime}$ & 6312.7 & 8.0 & 110 & 50934 \\
\hline 8 & 8 & 1975 & 1718 & $32^{\circ} 10.1^{\prime}$ & $-65^{\circ} 53.6^{\prime}$ & 6327.0 & 8.1 & 124 & 50920 \\
\hline 8 & 8 & 1975 & 1730 & $32^{\circ} 09.2^{\prime}$ & $-65^{\circ} 52.0^{\prime}$ & 6328.6 & 6.5 & 119 & 50839 \\
\hline 8 & 8 & 1975 & 1900 & $32^{\circ} 04.5^{\prime}$ & $-65^{\circ} 42.0^{\prime}$ & 6338.3 & 8.2 & 126 & 50784 \\
\hline 8 & 8 & 1975 & 1945 & $32^{\circ} 00.9^{\prime}$ & $-65^{\circ} 36.1^{\prime}$ & 6344.5 & 9.4 & 125 & 50607 \\
\hline 8 & 8 & 1975 & 2151 & $31^{\circ} 49.5^{\prime}$ & $-65^{\circ} 17.0^{\prime}$ & 6364.3 & 9.0 & 124 & 50451 \\
\hline 8 & 8 & 1975 & 2349 & $31^{\circ} 39.6^{\prime}$ & $-64^{\circ} 59.8^{\prime}$ & 6382.0 & 11.9 & 240 & 50451 \\
\hline 8 & 8 & 1975 & 2350 & $31^{\circ} 39.5^{\prime}$ & $-65^{\circ} 00.0^{\prime}$ & 6382.2 & 7.7 & 247 & 50451 \\
\hline 9 & 8 & 1975 & 000 & $31^{\circ} 39.0^{\prime}$ & $-65^{\circ} 01.4^{\prime}$ & 6383.5 & 7.8 & 250 & 50481 \\
\hline 9 & 8 & 1975 & 340 & $31^{\circ} 29.0^{\prime}$ & $-65^{\circ} 33.0^{\prime}$ & 6412.2 & 10.0 & 246 & 50489 \\
\hline 9 & 8 & 1975 & 613 & $31^{\circ} 18.7^{\prime}$ & $-66^{\circ} 00.4^{\prime}$ & 6437.7 & 9.6 & 301 & 50754 \\
\hline 9 & 8 & 1975 & 930 & $31^{\circ} 34.8^{\prime}$ & $-66^{\circ} 32.0^{\prime}$ & 6469.1 & 9.9 & 309 & 51215 \\
\hline 9 & 8 & 1975 & 1437 & $32^{\circ} 07.0^{\prime}$ & $-67^{\circ} 18.0^{\prime}$ & 6519.8 & 9.4 & 302 & 51325 \\
\hline 9 & 8 & 1975 & 1600 & $32^{\circ} 14.0^{\prime}$ & $-67^{\circ} 31.0^{\prime}$ & 6532.8 & 10.2 & 297 & 51445 \\
\hline 9 & 8 & 1975 & 1730 & $32^{\circ} 21.0^{\prime}$ & $-67^{\circ} 47.0^{\prime}$ & 6548.0 & 9.7 & 308 & 51531 \\
\hline 9 & 8 & 1975 & 1830 & $32^{\circ} 27.0^{\prime}$ & $-67^{\circ} 56.0^{\prime}$ & 6557.7 & 9.0 & 303 & 51942 \\
\hline 10 & 8 & 1975 & 000 & $32^{\circ} 54.0^{\prime}$ & $-68^{\circ} 45.0^{\prime}$ & 6607.0 & 10.0 & 303 & 52511 \\
\hline 10 & 8 & 1975 & 700 & $33^{\circ} 32.0^{\prime}$ & $-69^{\circ} 55.0^{\prime}$ & 6676.8 & 6.3 & 312 & 52650 \\
\hline 10 & 8 & 1975 & 930 & $33^{\circ} 42.6^{\prime}$ & $-70^{\circ} 08.9^{\prime}$ & 6692.5 & 6.8 & 305 & 53006 \\
\hline 10 & 8 & 1975 & 1600 & $34^{\circ} 07.7^{\prime}$ & $-70^{\circ} 52.5^{\prime}$ & 6736.6 & 9.0 & 302 & 53214 \\
\hline 10 & 8 & 1975 & 1900 & $34^{\circ} 22.0^{\prime}$ & $-71^{\circ} 20.0^{\prime}$ & 6763.4 & 8.8 & 305 & 53557 \\
\hline 11 & 8 & 1975 & 000 & $34^{\circ} 46.8^{\prime}$ & $-72^{\circ} 03.8^{\prime}$ & 6807.2 & 8.7 & 305 & 53628 \\
\hline 11 & 8 & 1975 & 103 & $34^{\circ} 52.0^{\prime}$ & $-72^{\circ} 13.0^{\prime}$ & 6816.4 & 10.6 & 302 & 53810 \\
\hline 11 & 8 & 1975 & 320 & $35^{\circ} 05.0^{\prime}$ & $-72^{\circ} 38.0^{\prime}$ & 6840.6 & 7.9 & 314 & 53942 \\
\hline 11 & 8 & 1975 & 520 & $35^{\circ} 16.0^{\prime}$ & $-72^{\circ} 52.0^{\prime}$ & 6856.5 & 7.7 & 300 & 54041 \\
\hline 11 & 8 & 1975 & 708 & $35^{\circ} 22.9^{\prime}$ & $-73^{\circ} 06.8^{\prime}$ & 6870.4 & 5.8 & 302 & 54042 \\
\hline 11 & 8 & 1975 & 710 & $35^{\circ} 23.0^{\prime}$ & $-73^{\circ} 07.0^{\prime}$ & 6870.6 & 5.6 & 334 & 54052 \\
\hline 11 & 8 & 1975 & 722 & $35^{\circ} 24.0^{\prime}$ & $-73^{\circ} 07.6^{\prime}$ & 6871.7 & 7.3 & 326 & 54059 \\
\hline 11 & 8 & 1975 & 728 & $35^{\circ} 24.6^{\prime}$ & $-73^{\circ} 08.1^{\prime}$ & 6872.4 & 7.0 & 328 & 54217 \\
\hline 11 & 8 & 1975 & 1000 & $35^{\circ} 39.6^{\prime}$ & $-73^{\circ} 19.7^{\prime}$ & 6890.1 & 7.9 & 325 & 54508 \\
\hline 11 & 8 & 1975 & 1415 & $36^{\circ} 07.0^{\prime}$ & $-73^{\circ} 43.5^{\prime}$ & 6923.6 & 7.4 & 314 & 54612 \\
\hline 11 & 8 & 1975 & 1600 & $36^{\circ} 16.0^{\prime}$ & $-73^{\circ} 55.0^{\prime}$ & 6936.6 & 11.6 & 296 & 54621 \\
\hline 11 & 8 & 1975 & 1607 & $36^{\circ} 16.6^{\prime}$ & $-73^{\circ} 56.5^{\prime}$ & 6937.9 & 10.8 & 297 & 54756 \\
\hline 11 & 8 & 1975 & 1800 & $36^{\circ} 26.0^{\prime}$ & $-74^{\circ} 19.0^{\prime}$ & 6958.3 & 7.1 & 309 & 54864 \\
\hline 11 & 8 & 1975 & 2000 & $36^{\circ} 35.0^{\prime}$ & $-74^{\circ} 32.7^{\prime}$ & 6972.6 & 5.3 & 313 & 54895 \\
\hline 11 & 8 & 1975 & 2045 & $36^{\circ} 37.7^{\prime}$ & $-74^{\circ} 36.3^{\prime}$ & 6976.5 & 5.0 & 302 & 54938 \\
\hline 11 & 8 & 1975 & 2200 & $36^{\circ} 41.0^{\prime}$ & $-74^{\circ} 43.0^{\prime}$ & 6982.8 & & & 54938 \\
\hline
\end{tabular}

${ }^{\mathrm{a}}$ All times are Greenwich Mean Time (Z). 


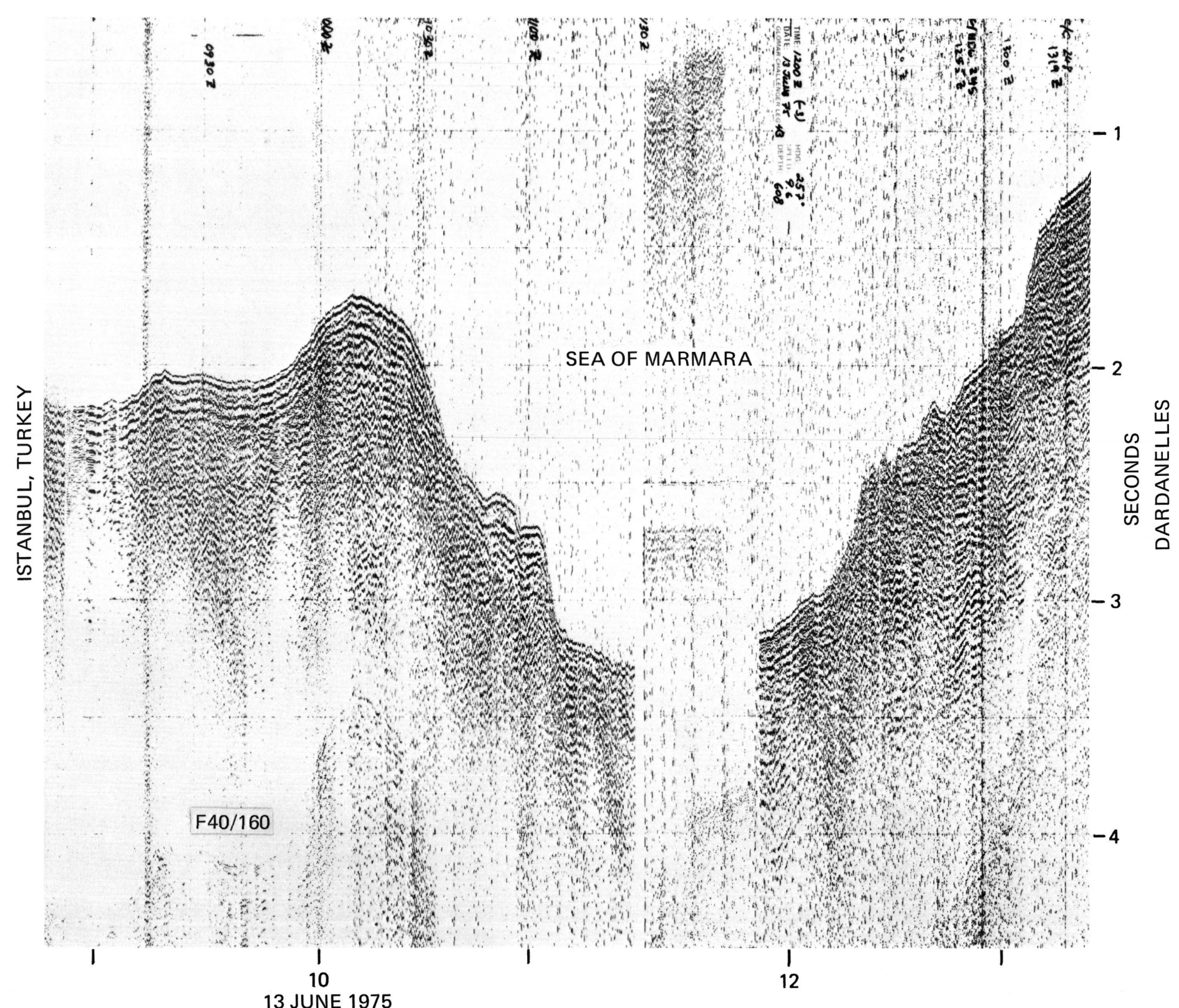

Figure 2. Glomar Challenger seismic profiles along Leg 43 track (see text for explanation of annotations). 


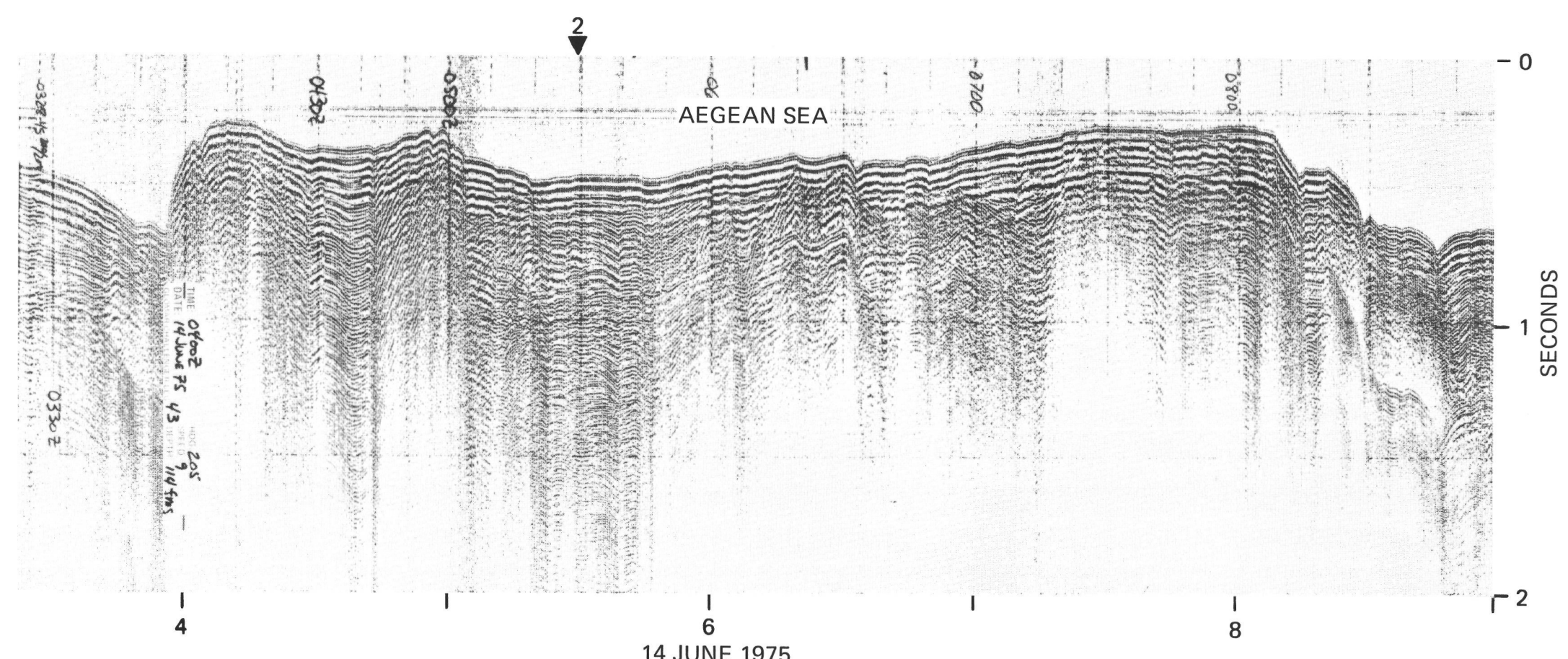

Figure 2. (Continued).

\section{JUNE 1975}




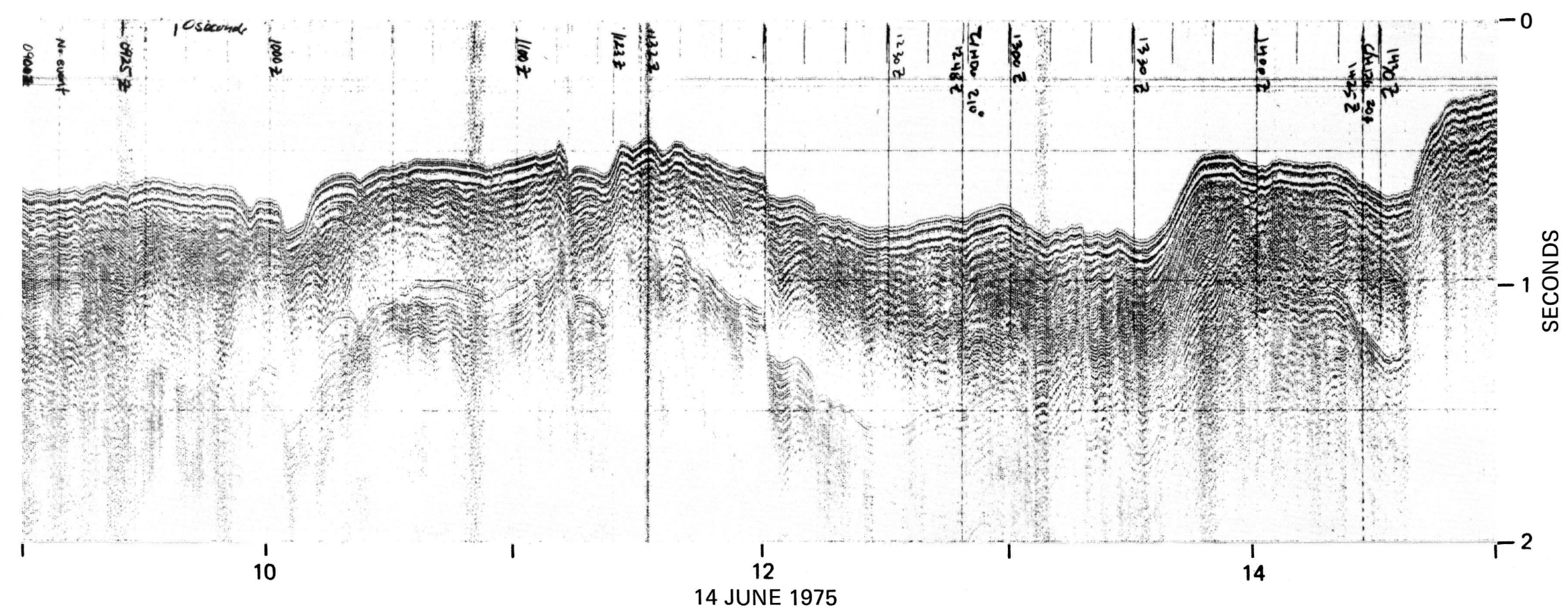

Figure 2. (Continued). 


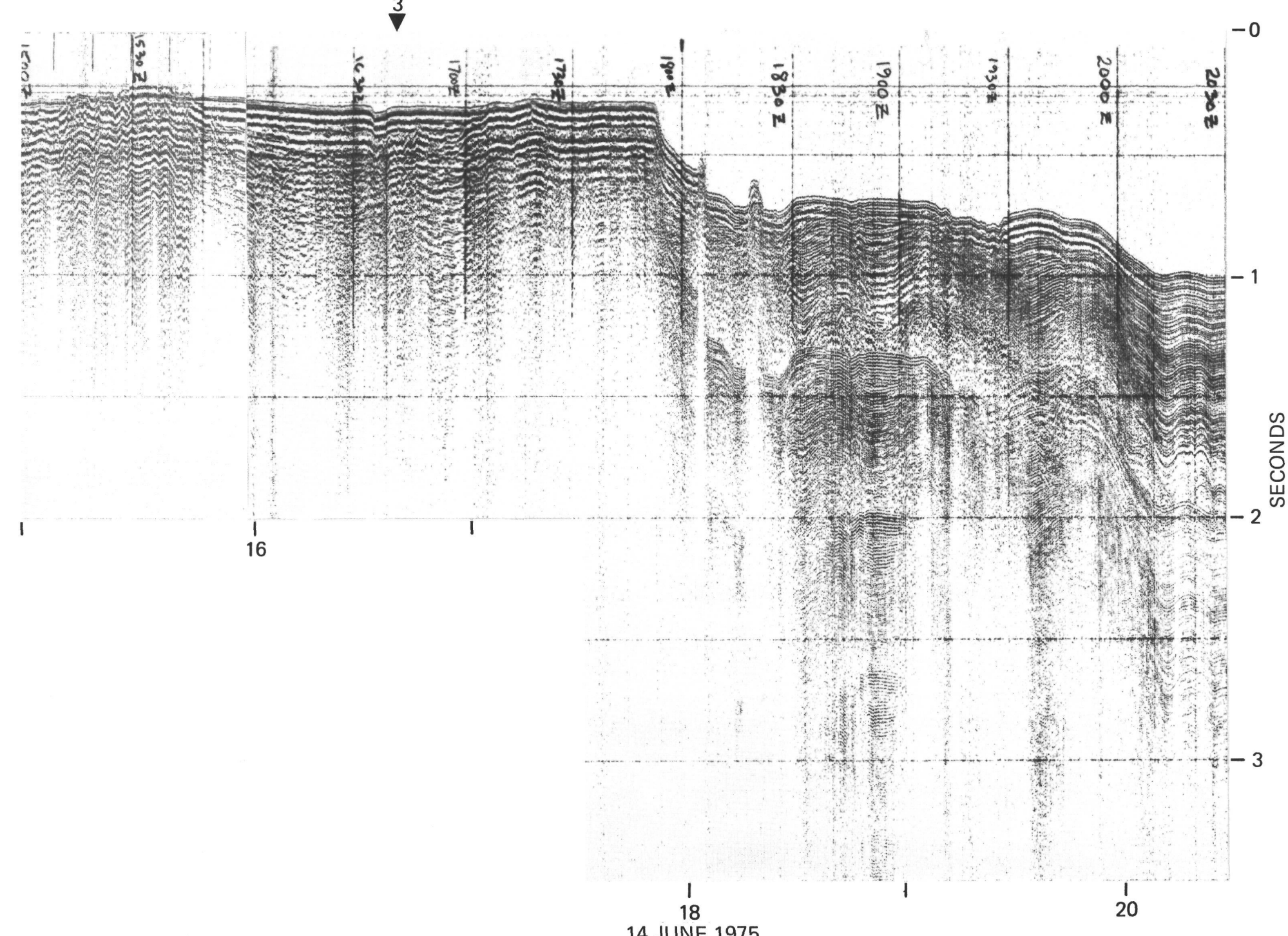

Figure 2. (Continued). 


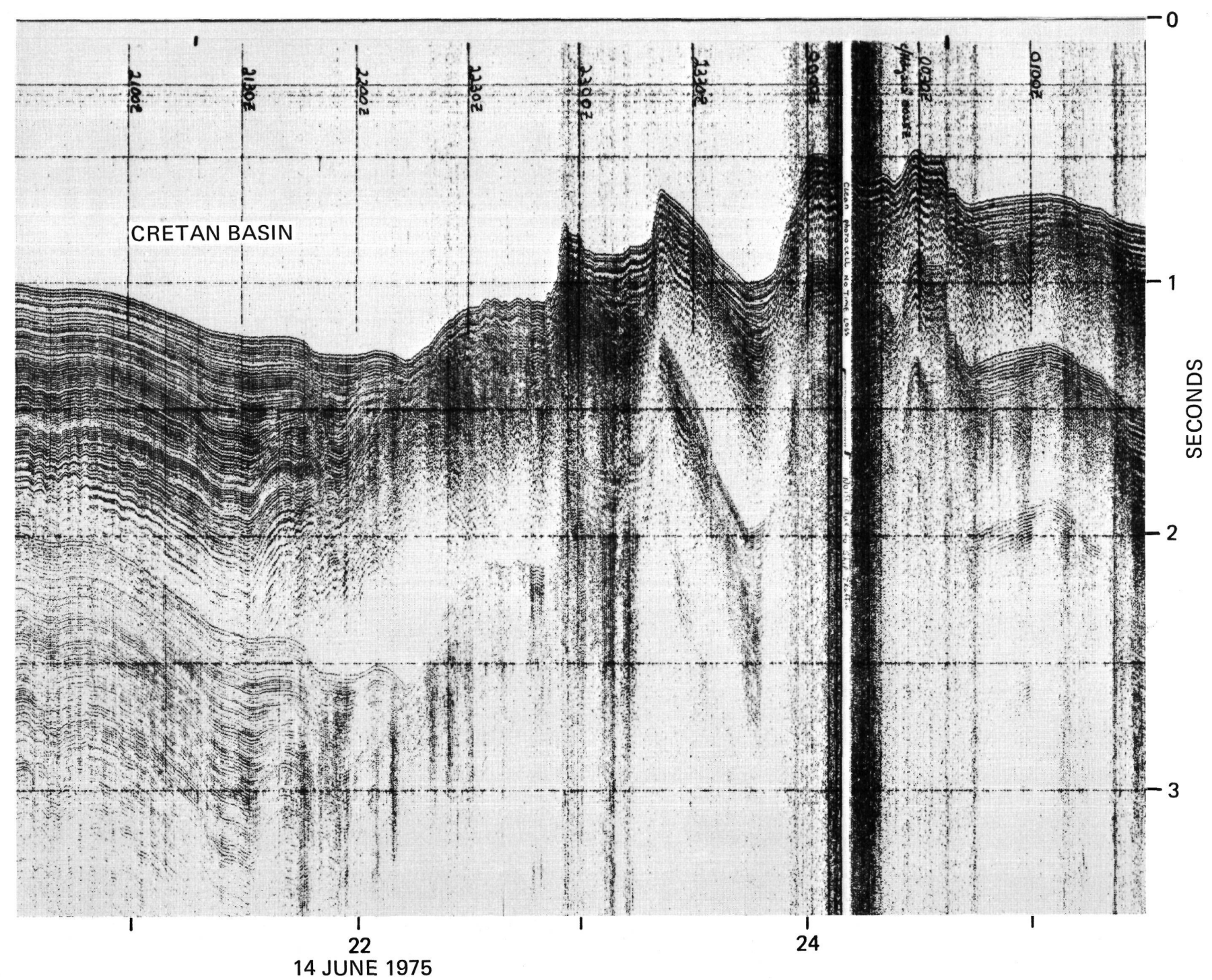

Figure 2. (Continued). 


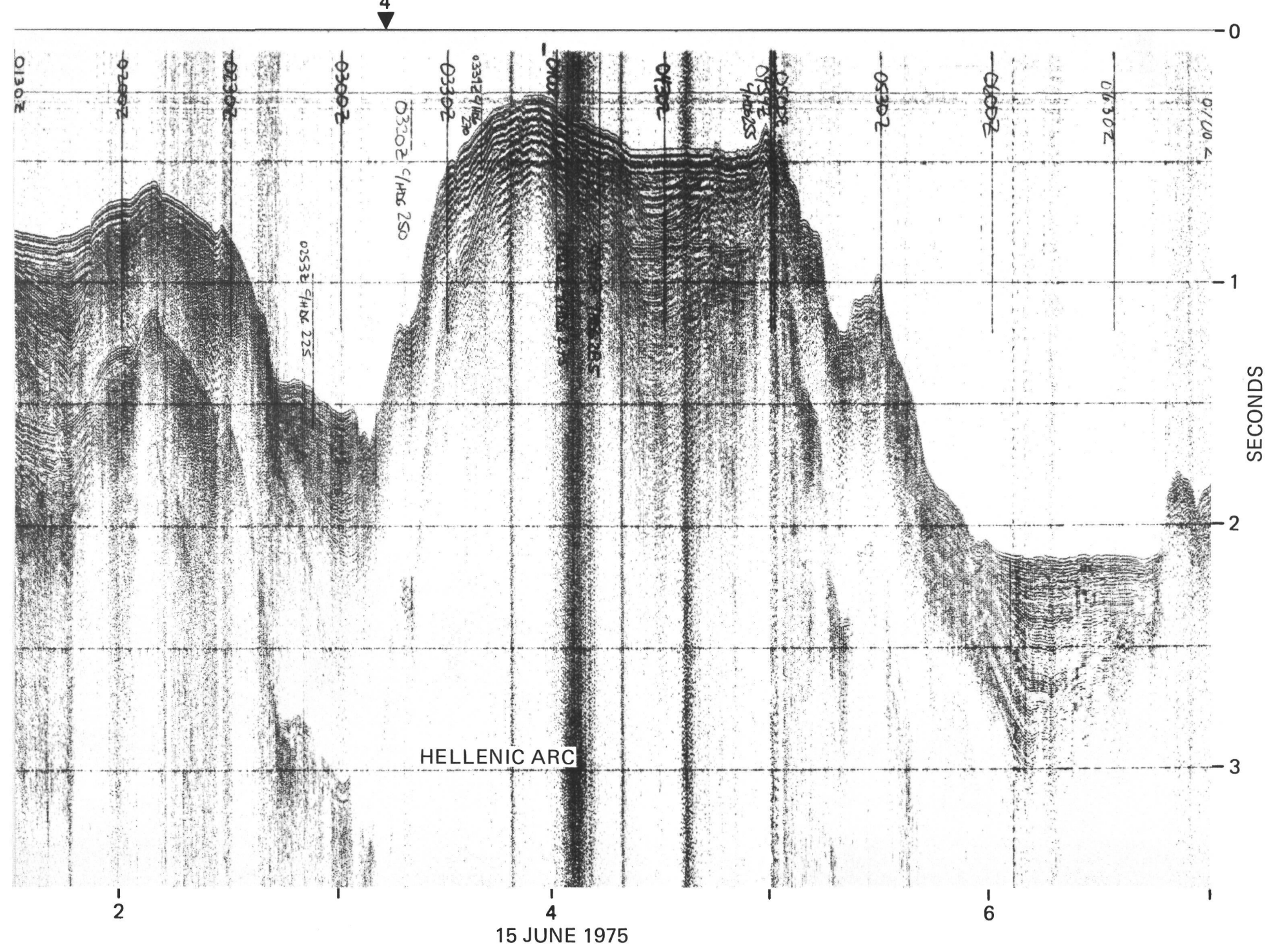

Figure 2. (Continued). 


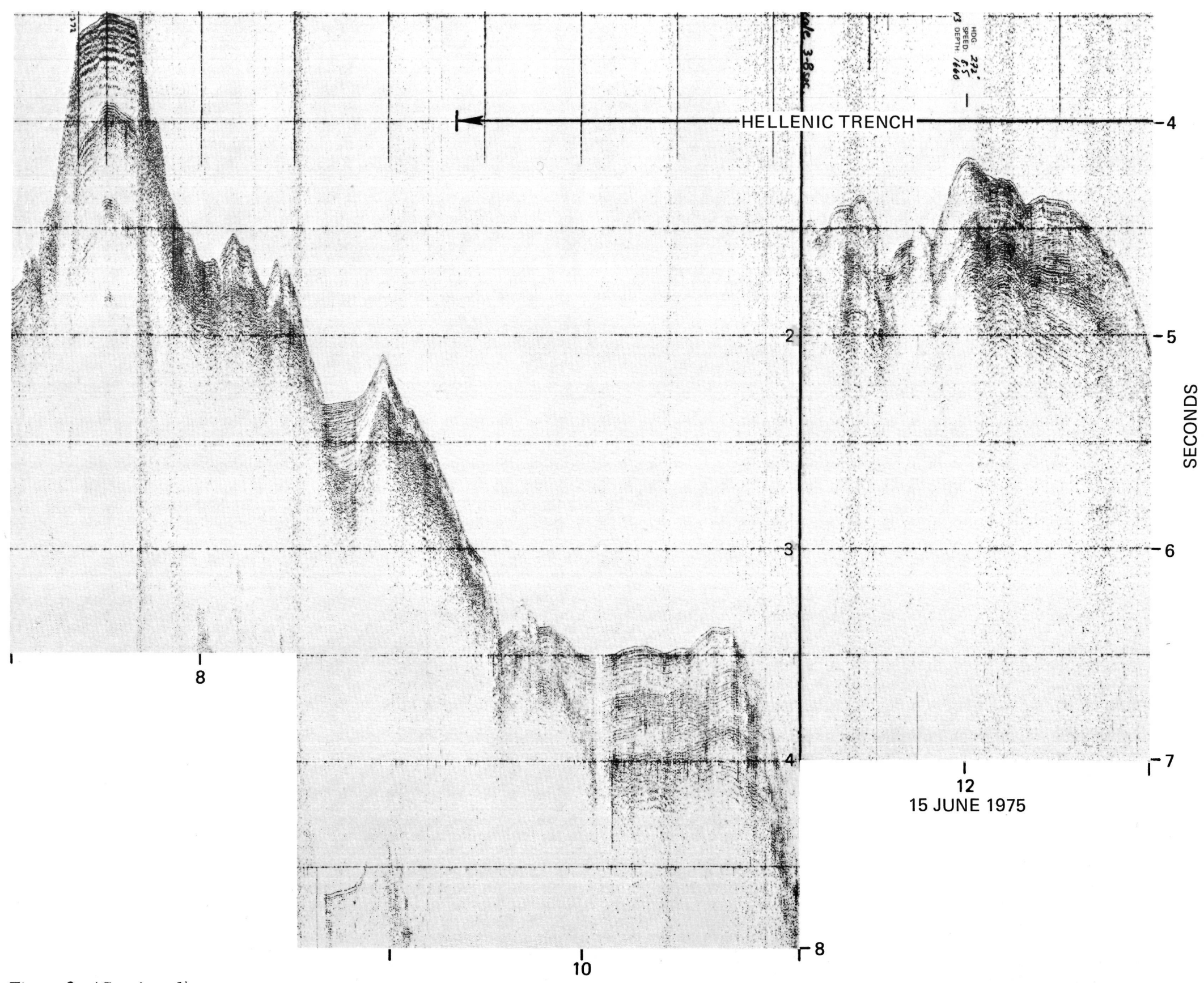




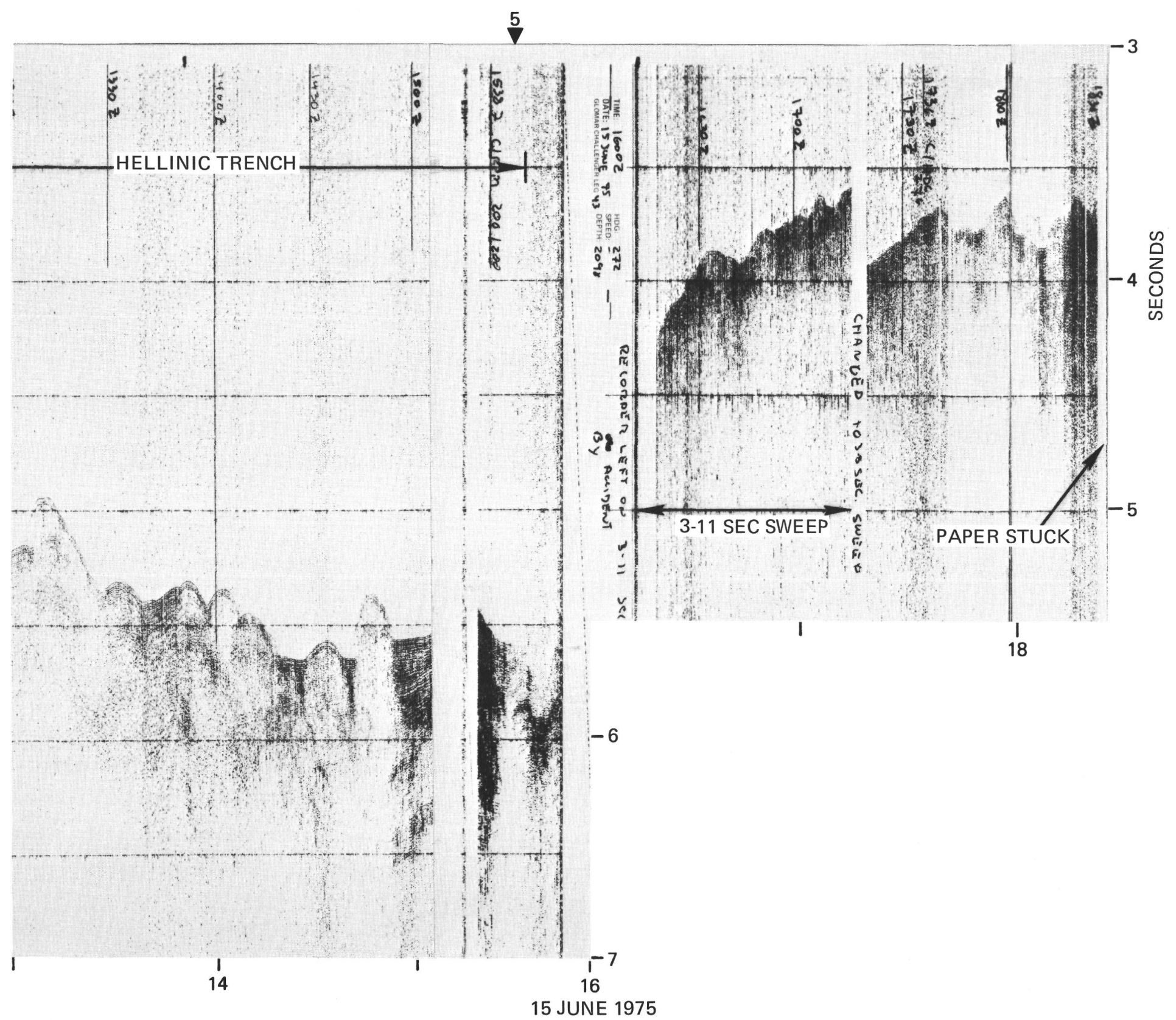

Figure 2. (Continued) 


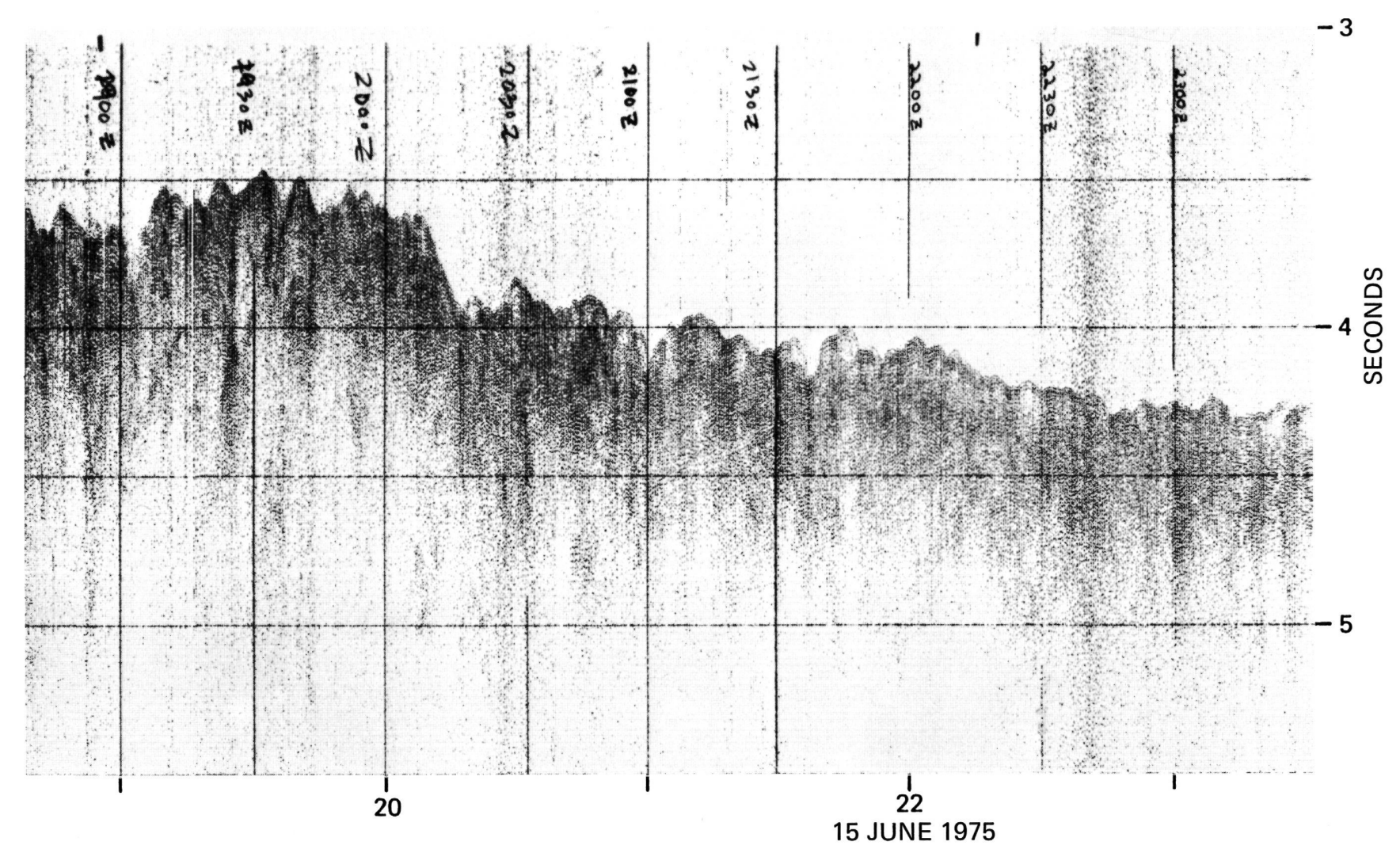

Figure 2. (Continued). 


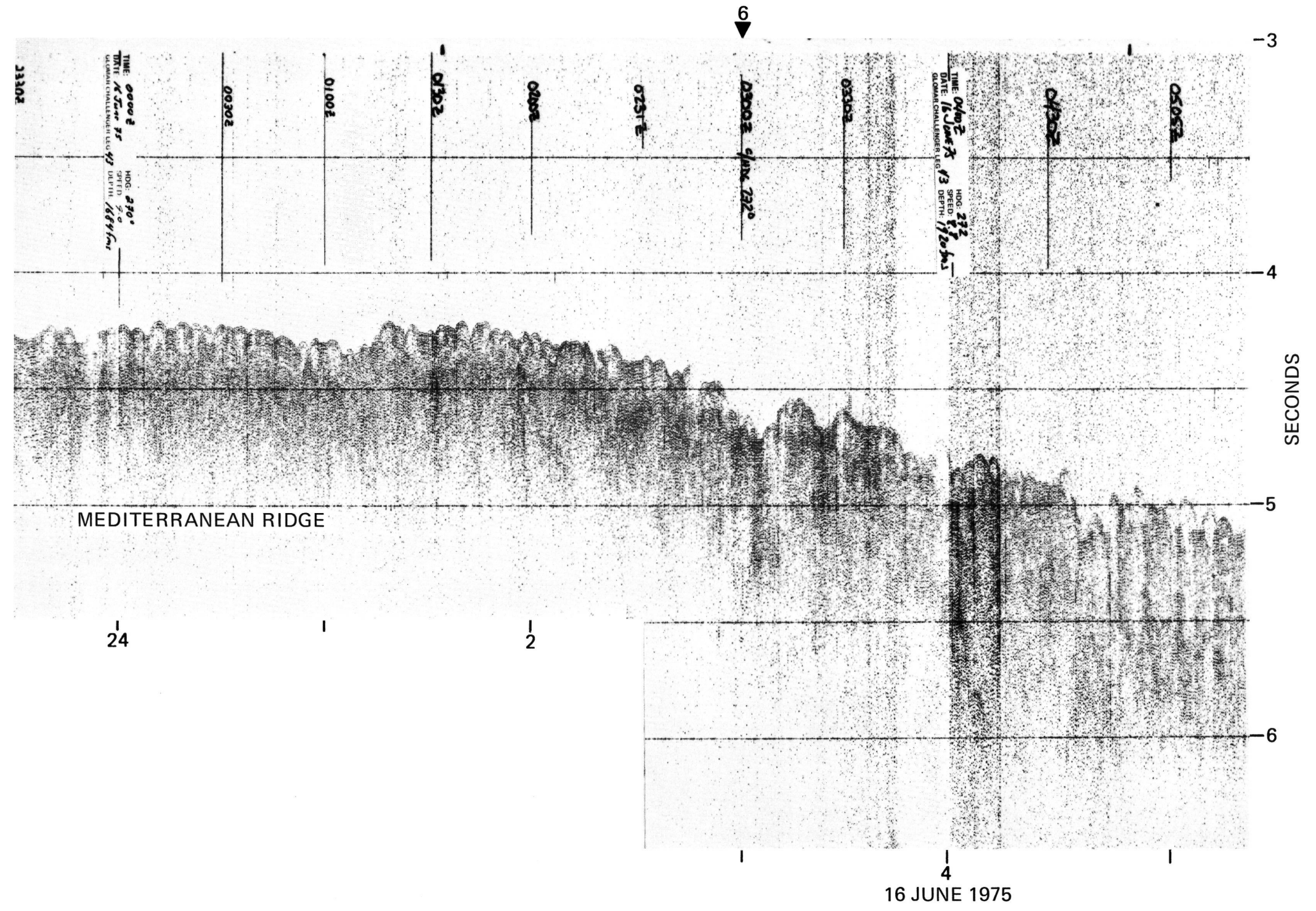

Figure 2. (Continued). 


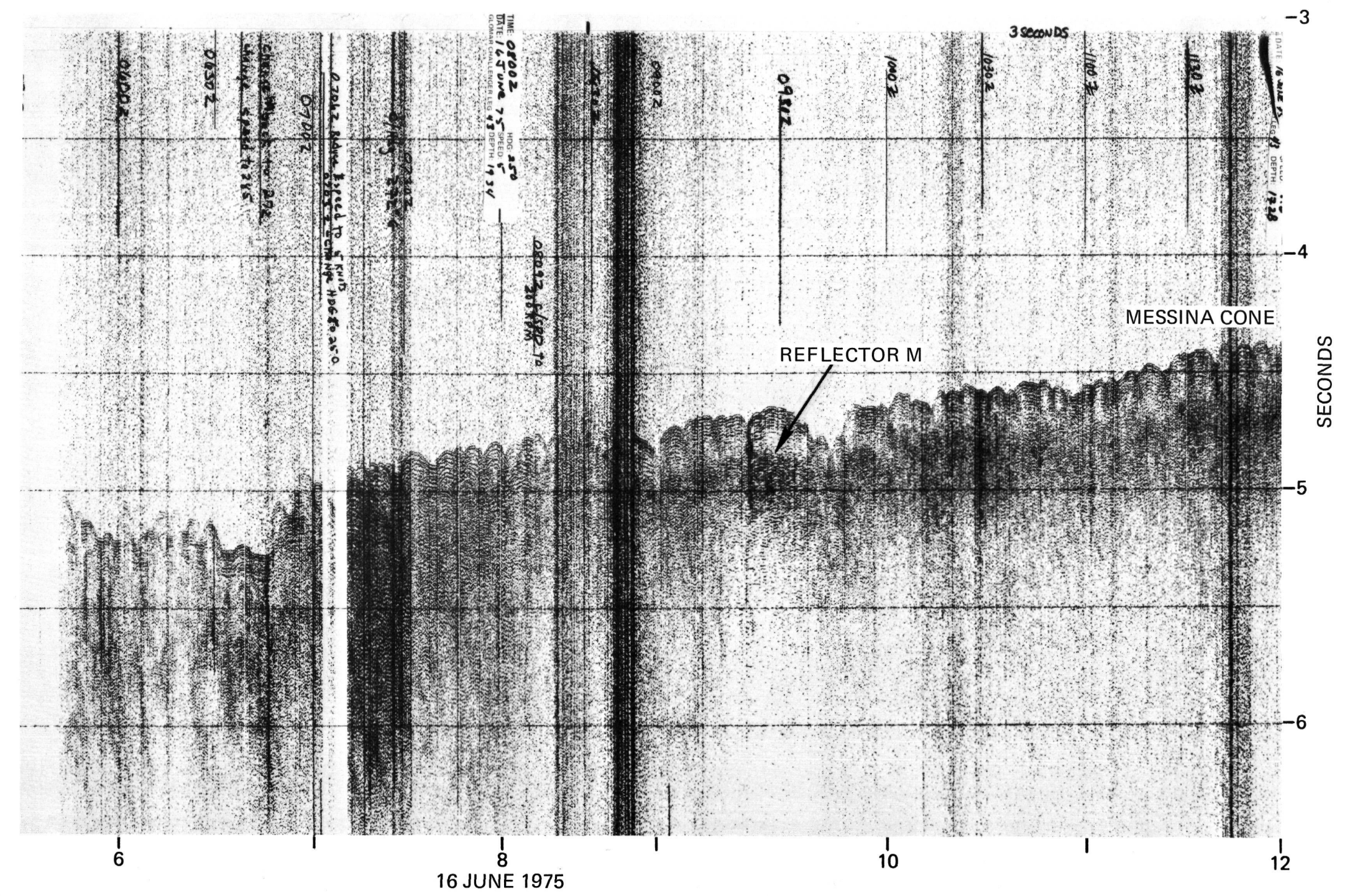

Figure 2. (Continued). 


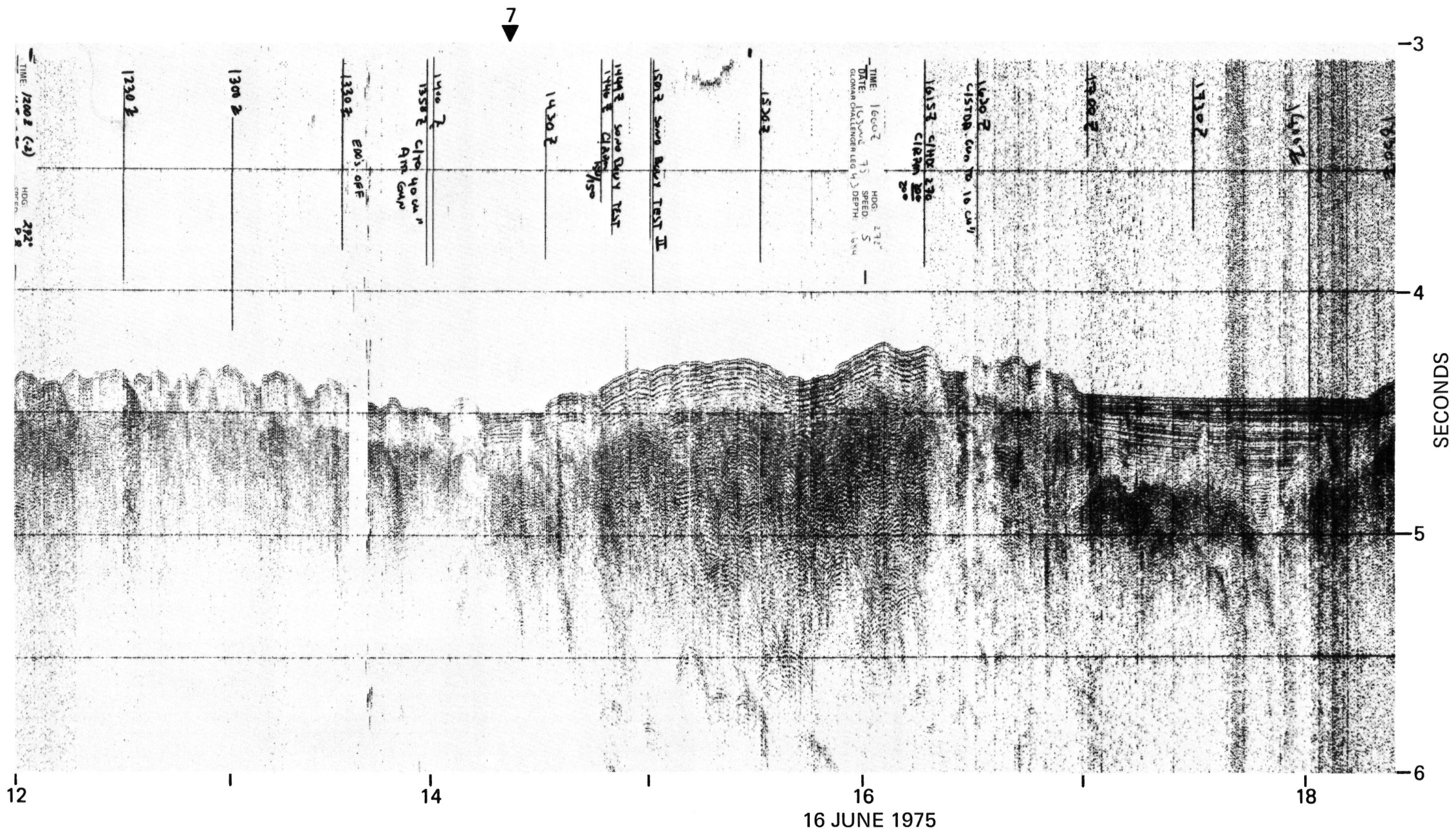

Figure 2. (Continued). 


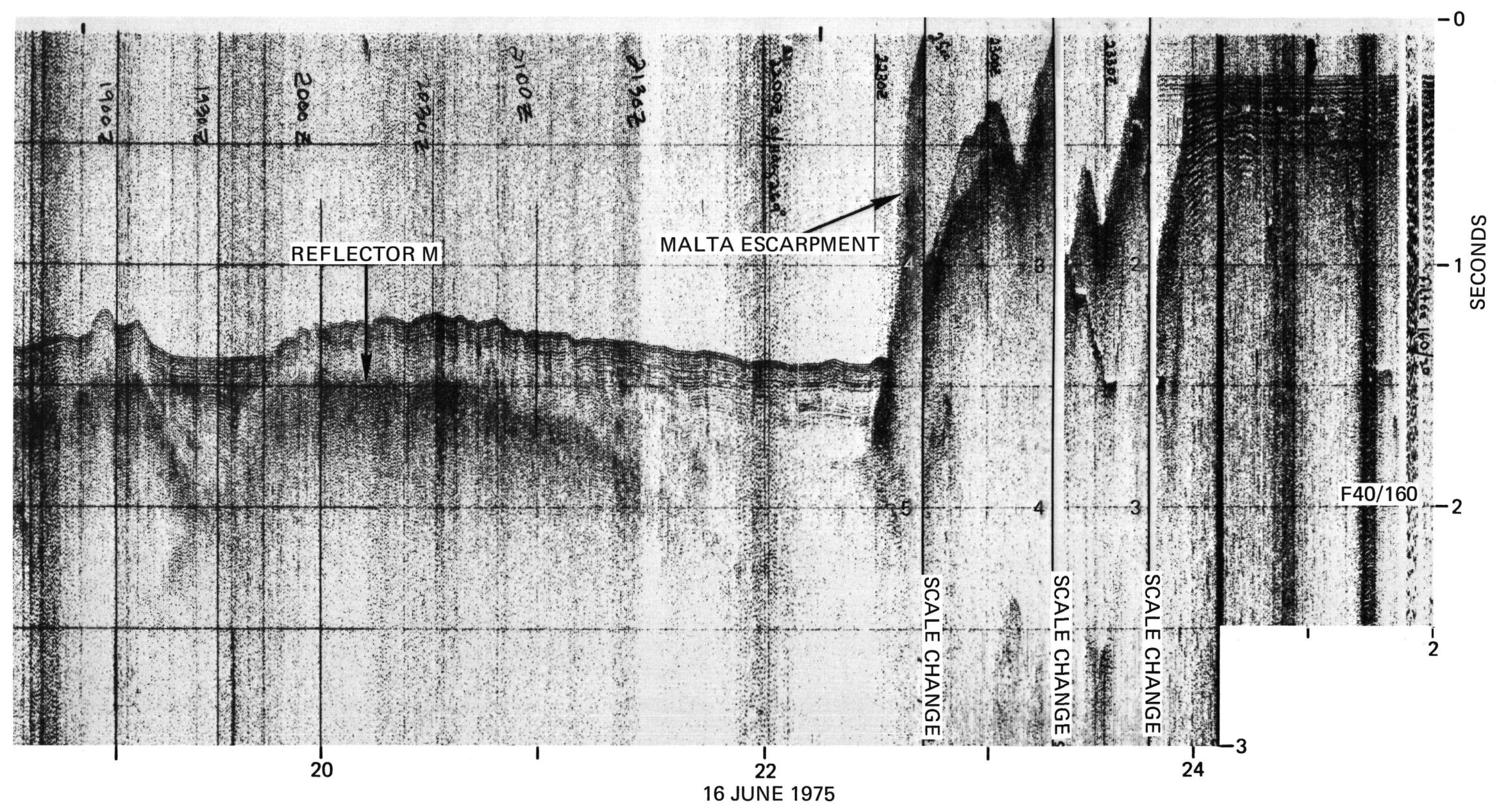

Figure 2. (Continued). 


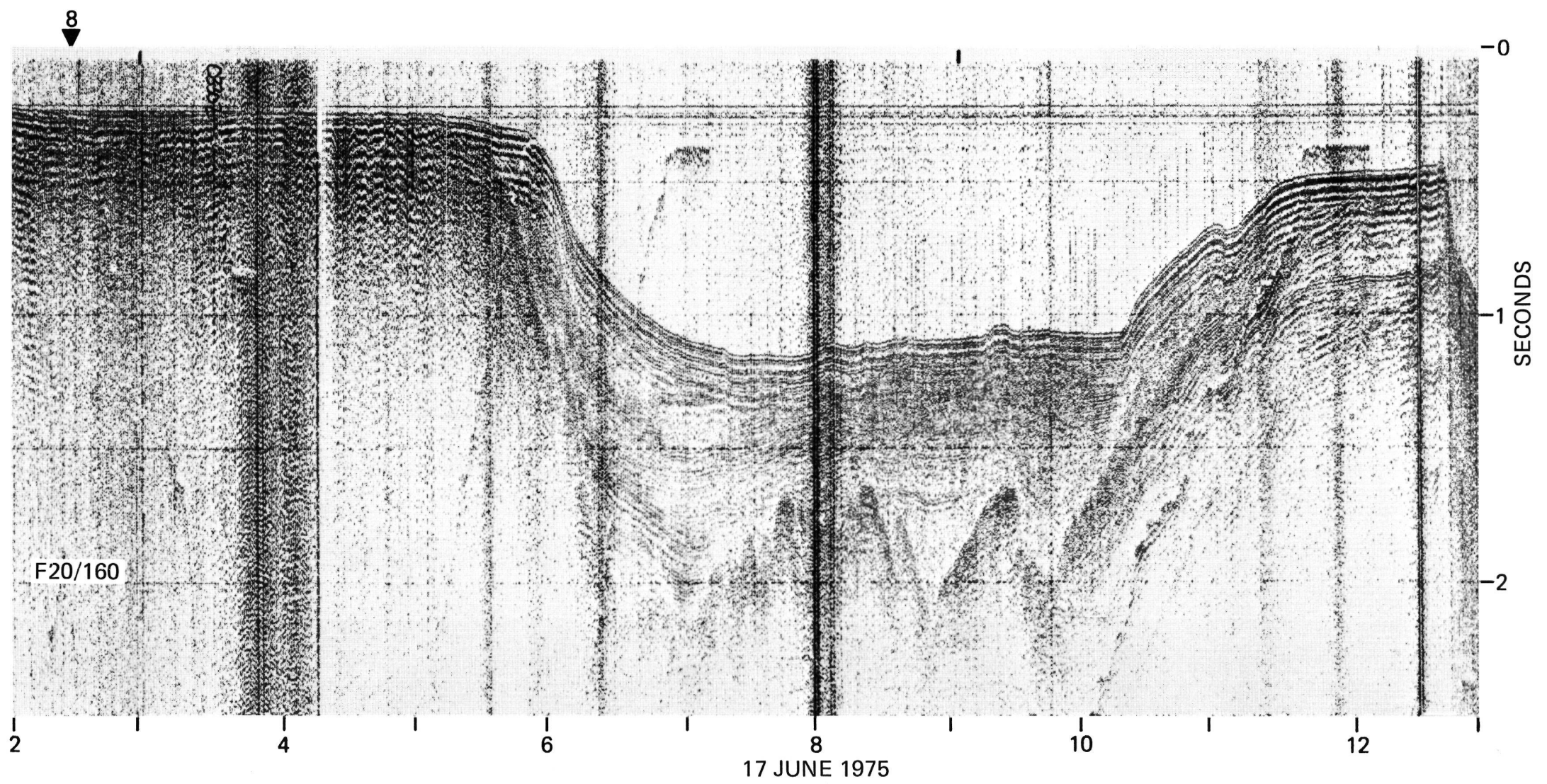

Figure 2. (Continued). 


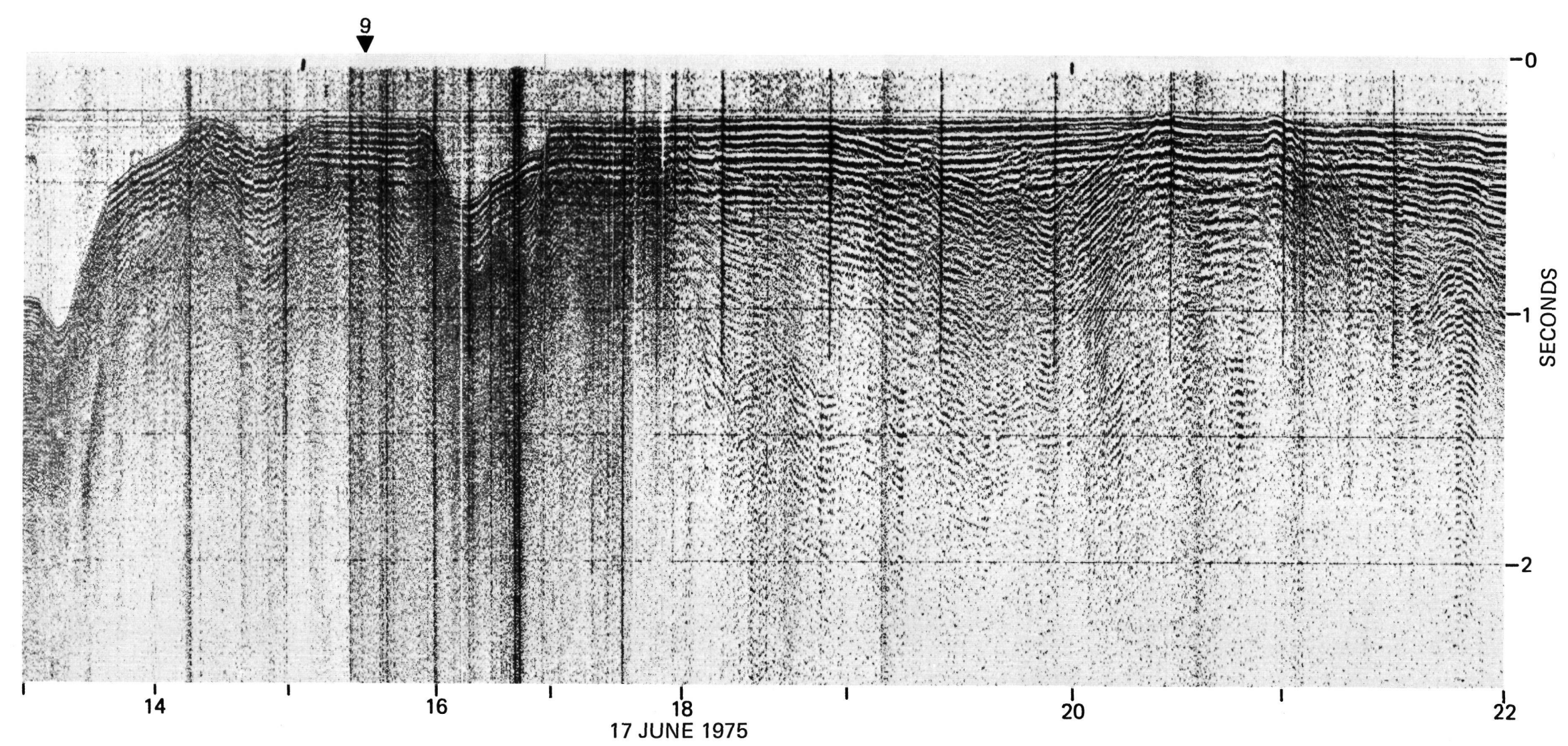

Figure 2. (Continued). 


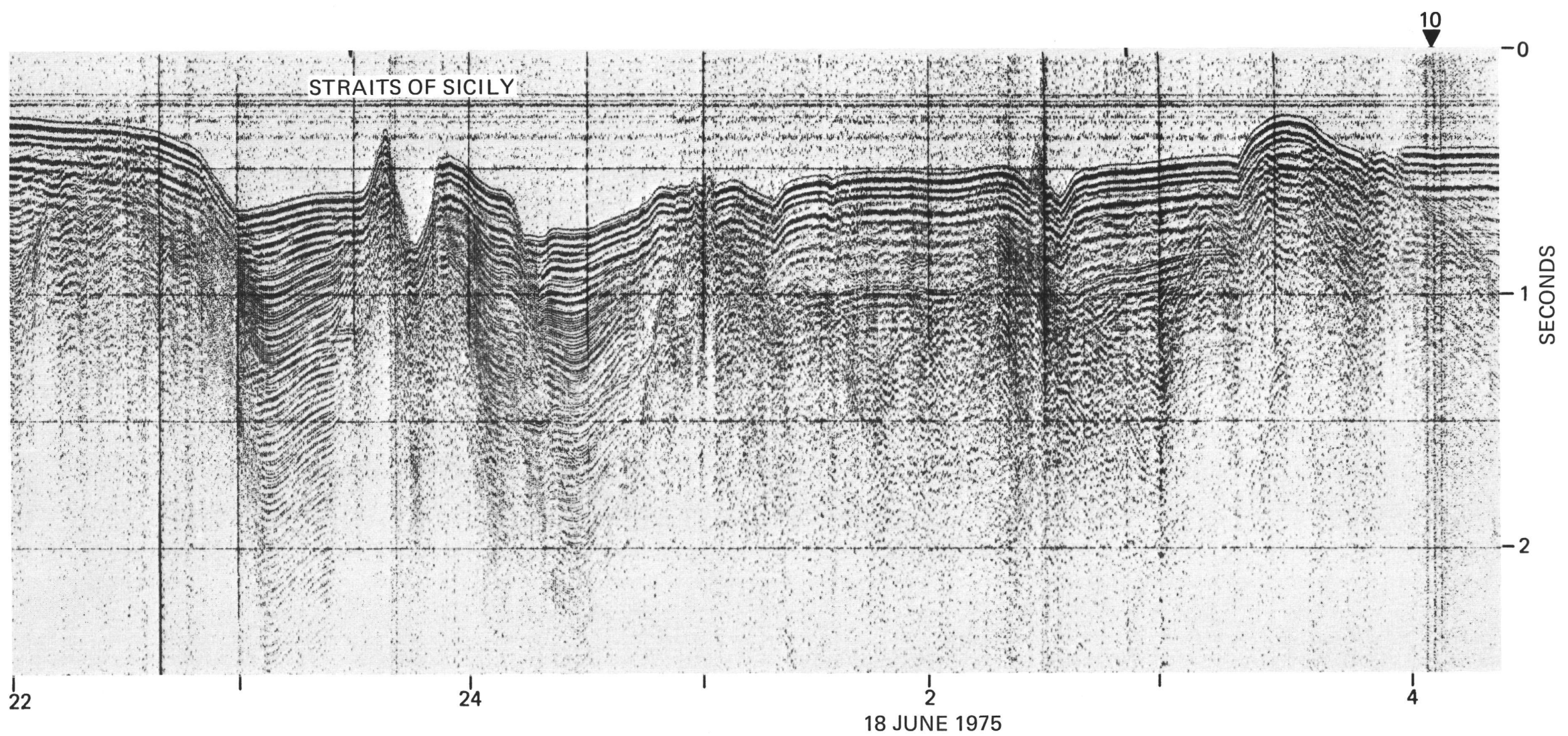

Figure 2. (Continued). 


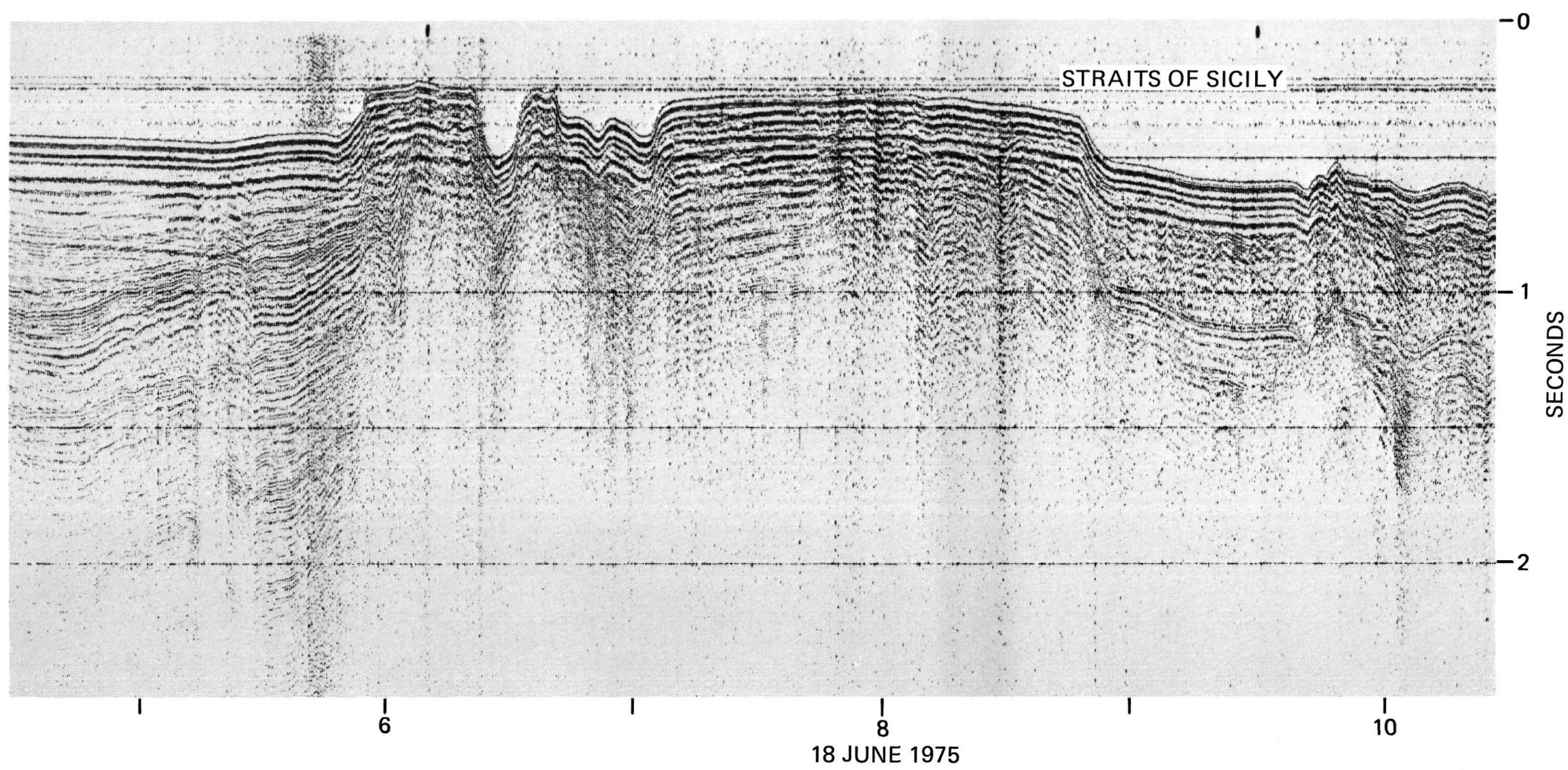

Figure 2. (Continued). 

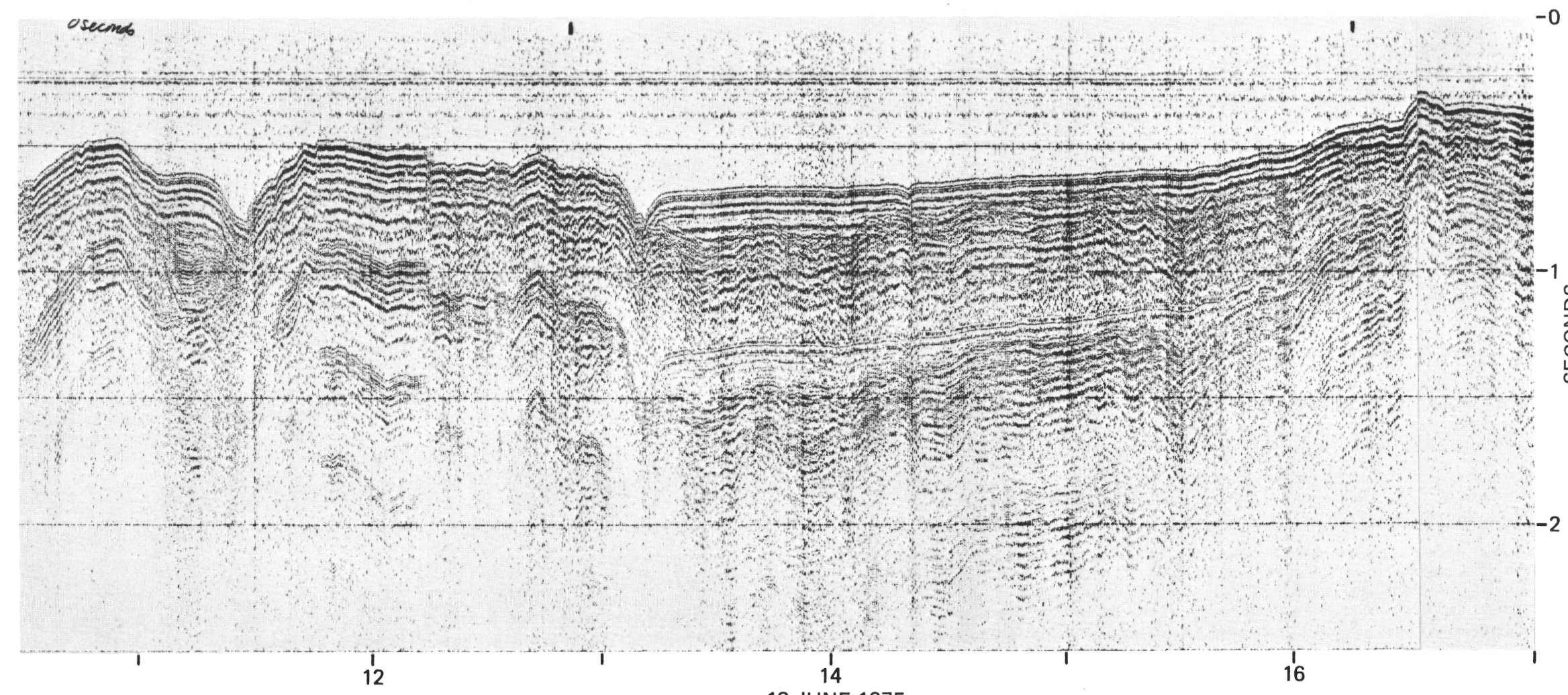

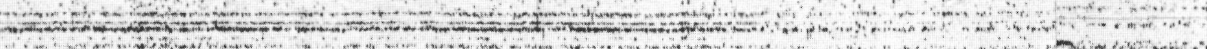

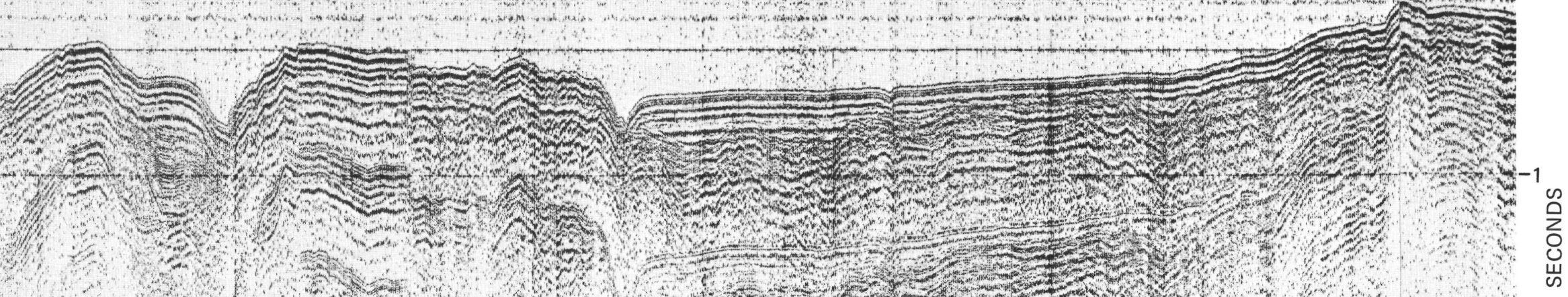

Figure 2. (Continued). 


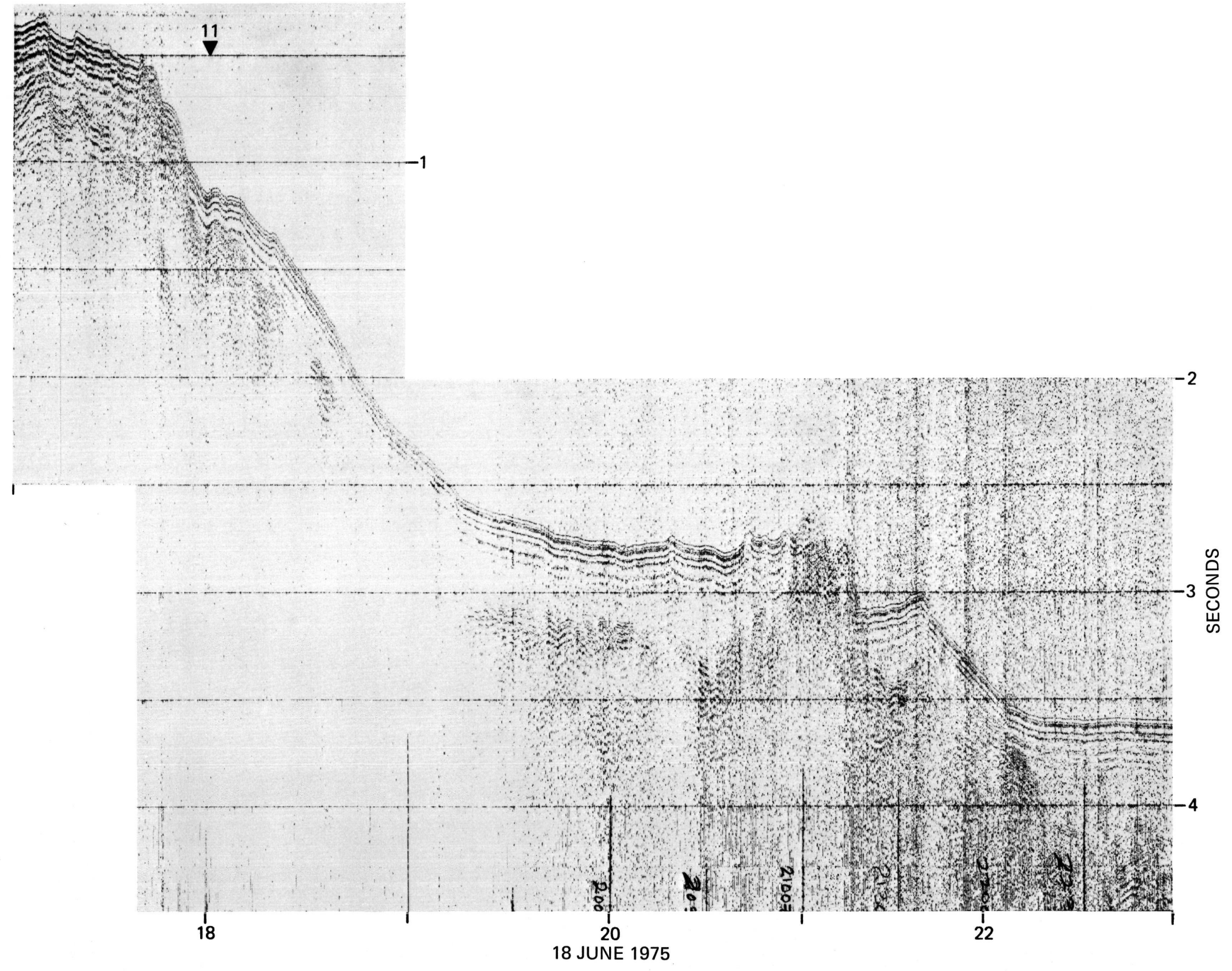




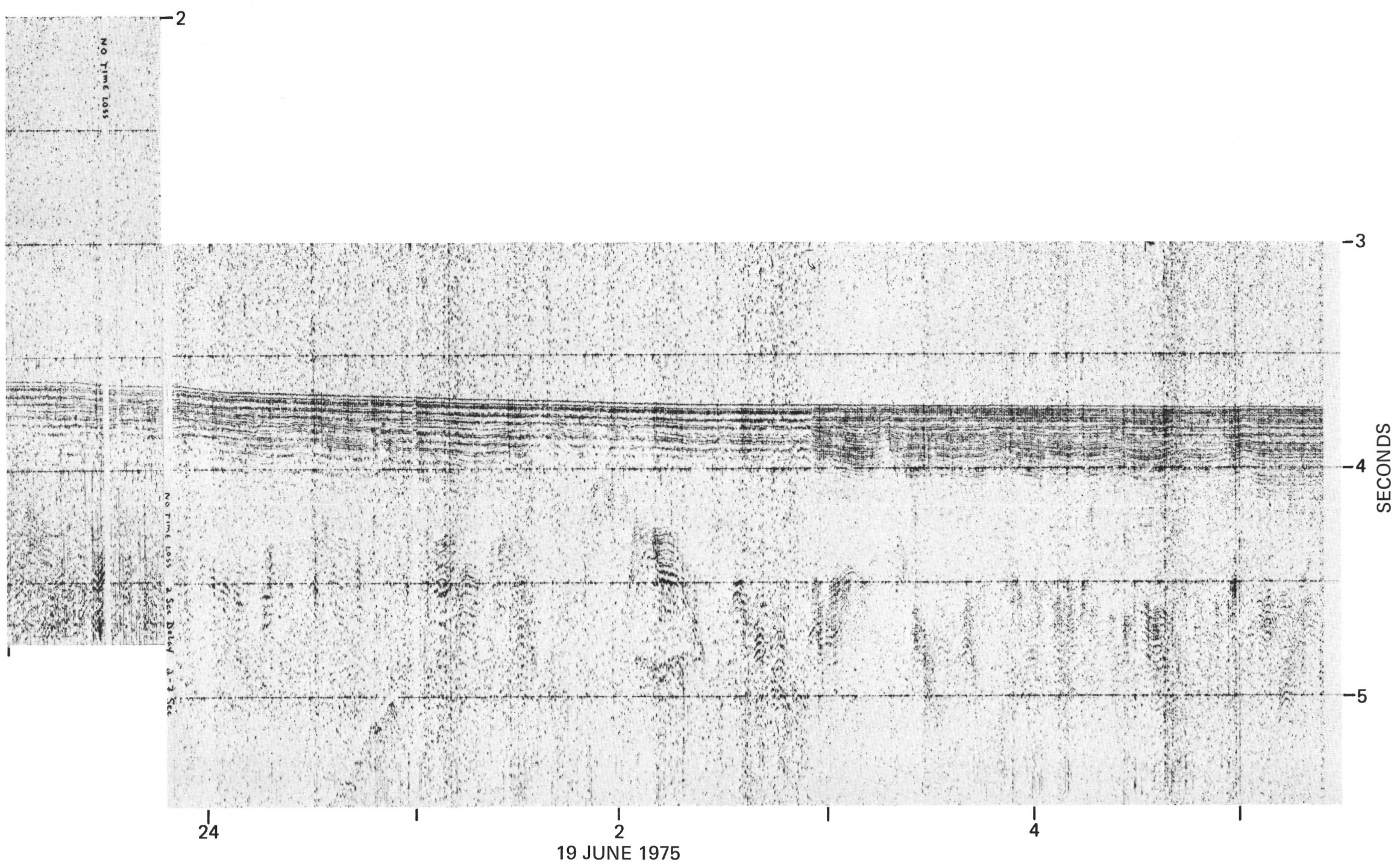

Figure 2. (Continued). 

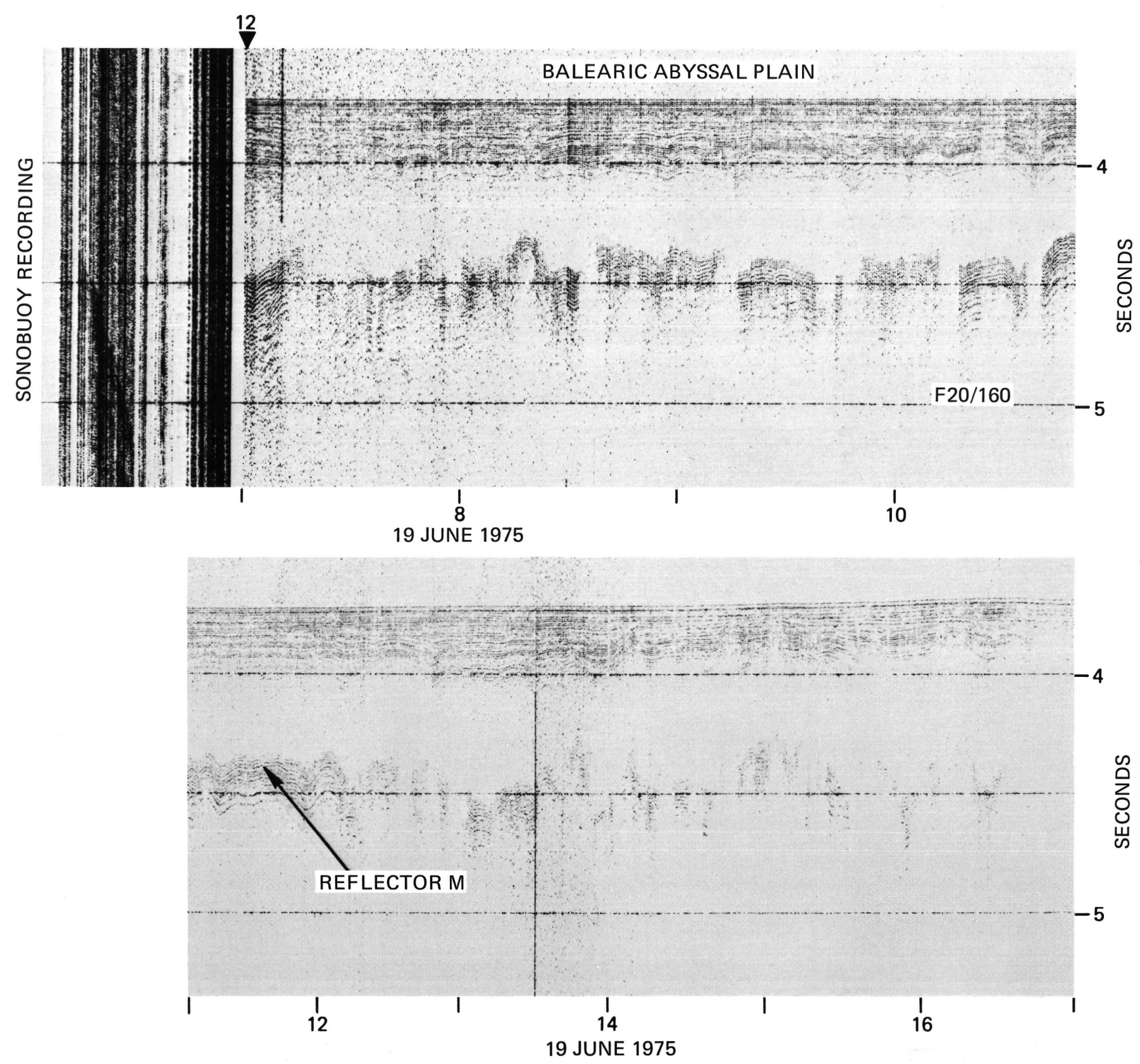

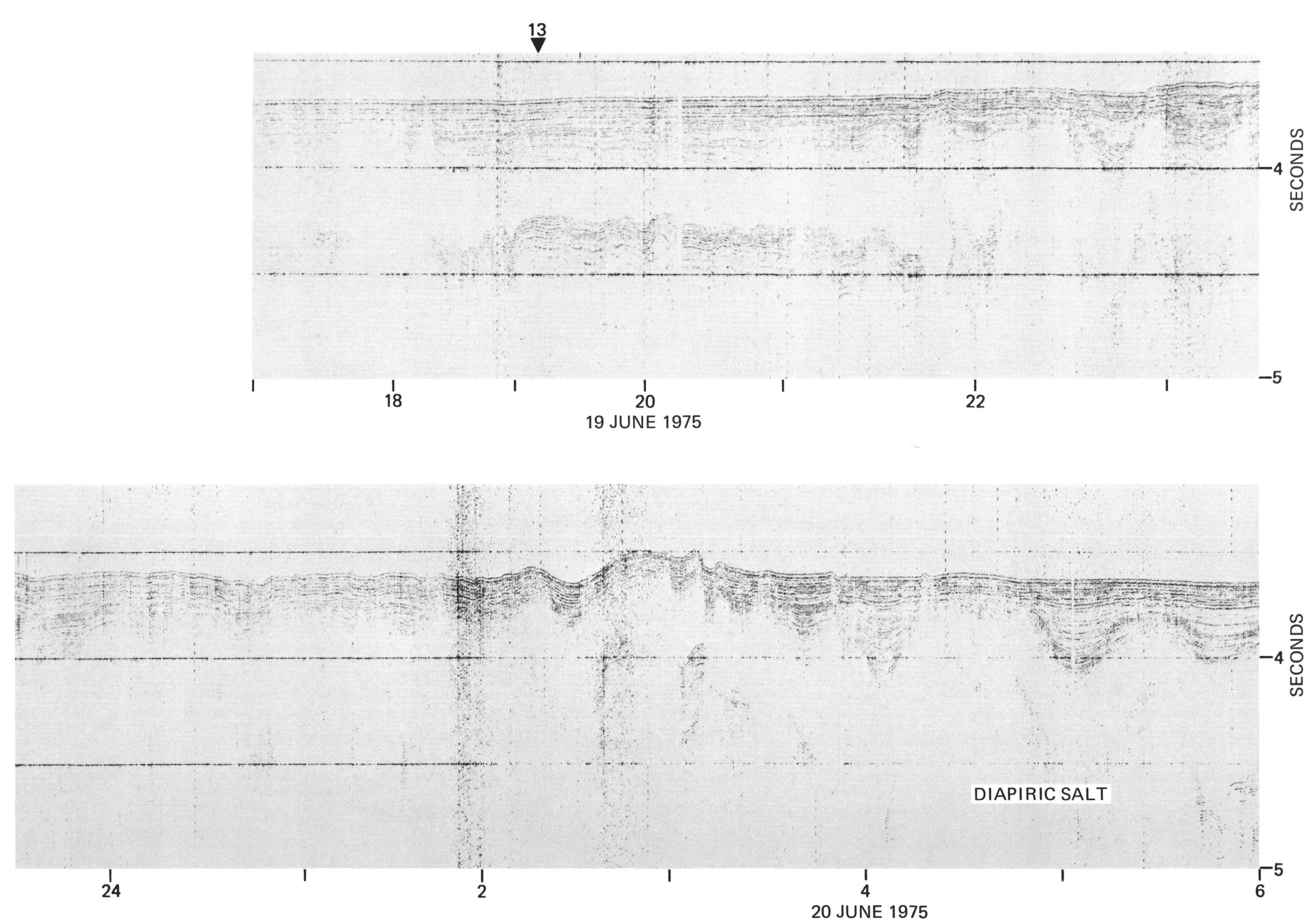

Figure 2. (Continued). 


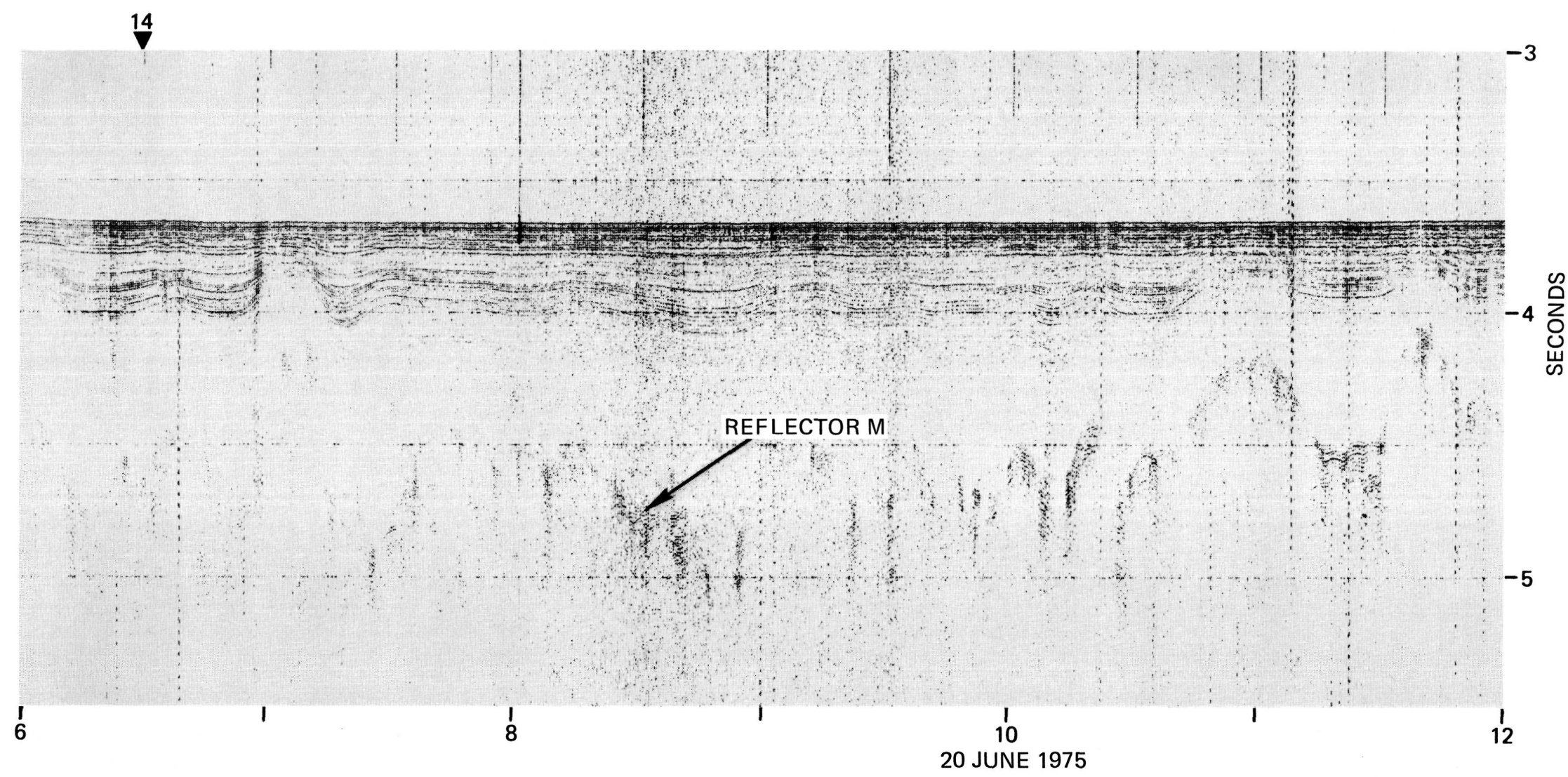

Figure 2. (Continued). 


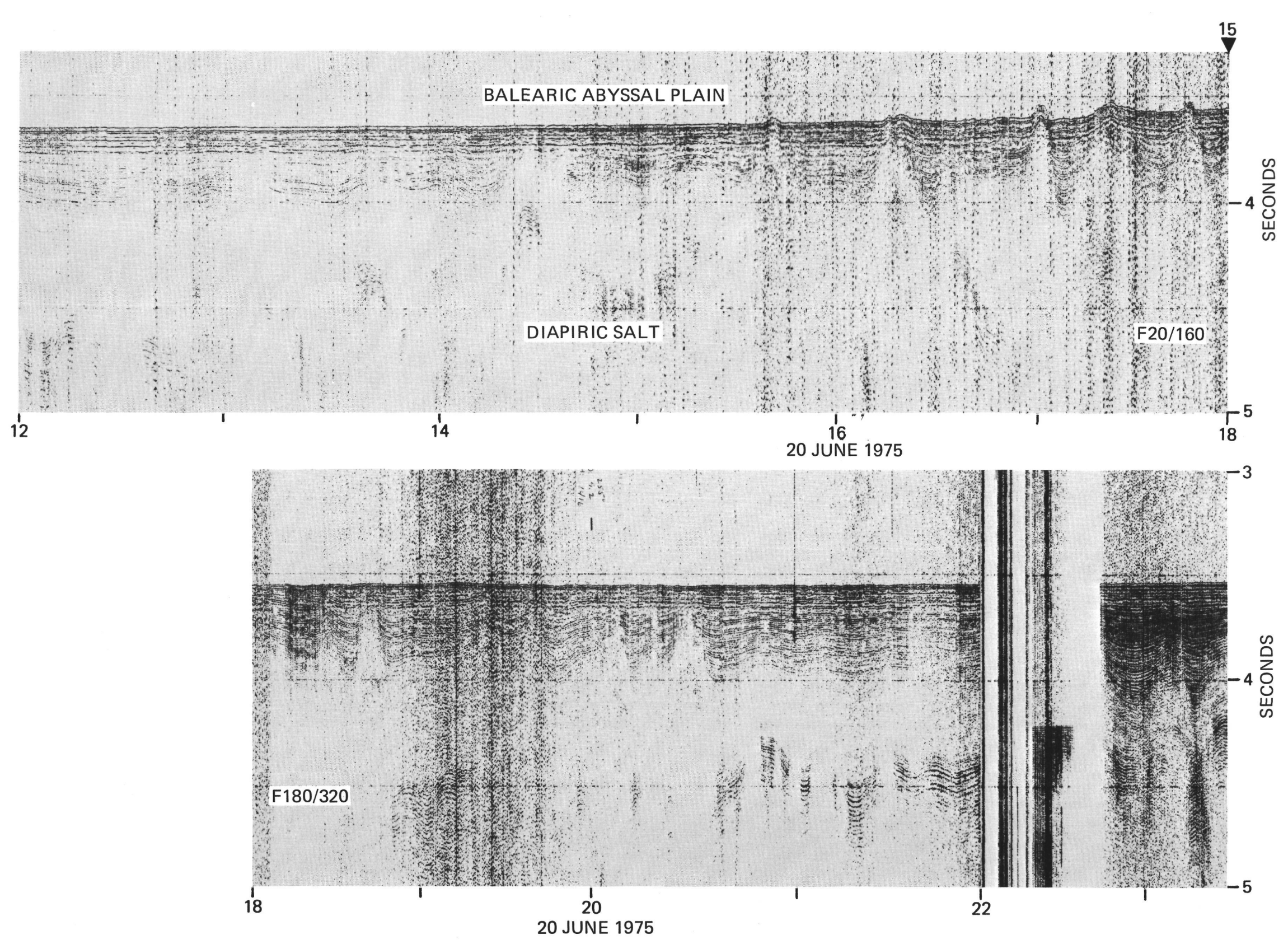

Figure 2. (Continued). 


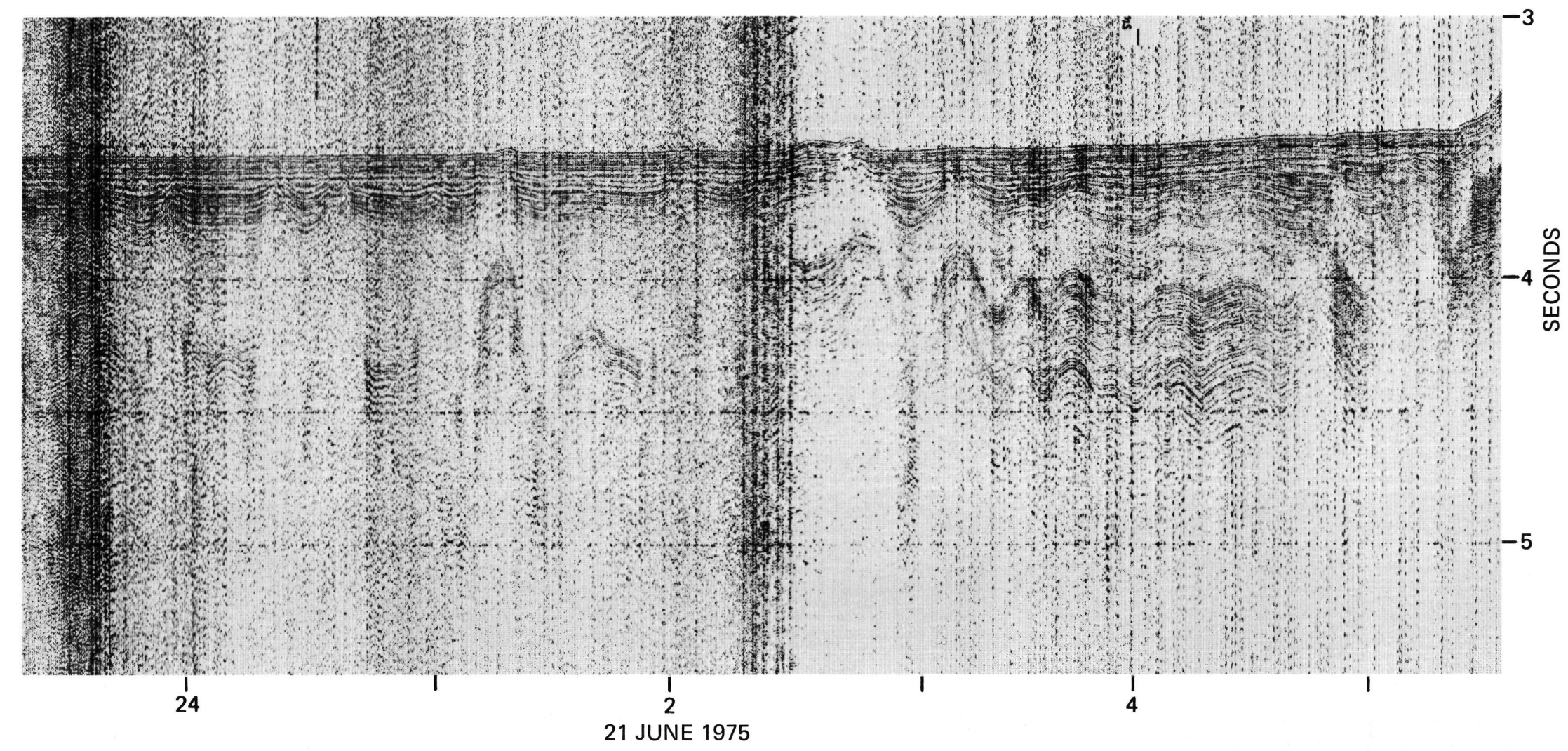

Figure 2. (Continued). 

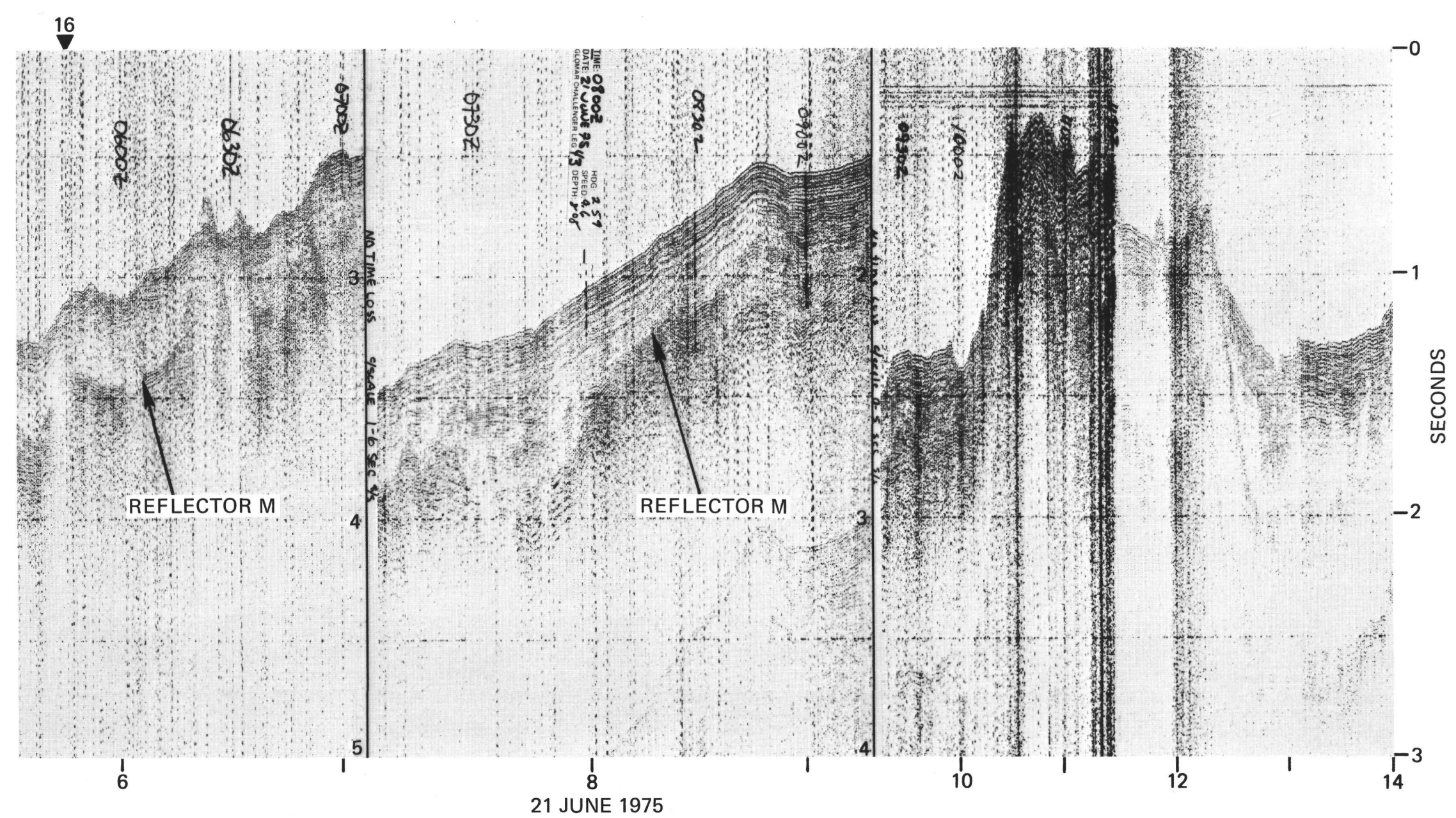

Figure 2. (Continued). 

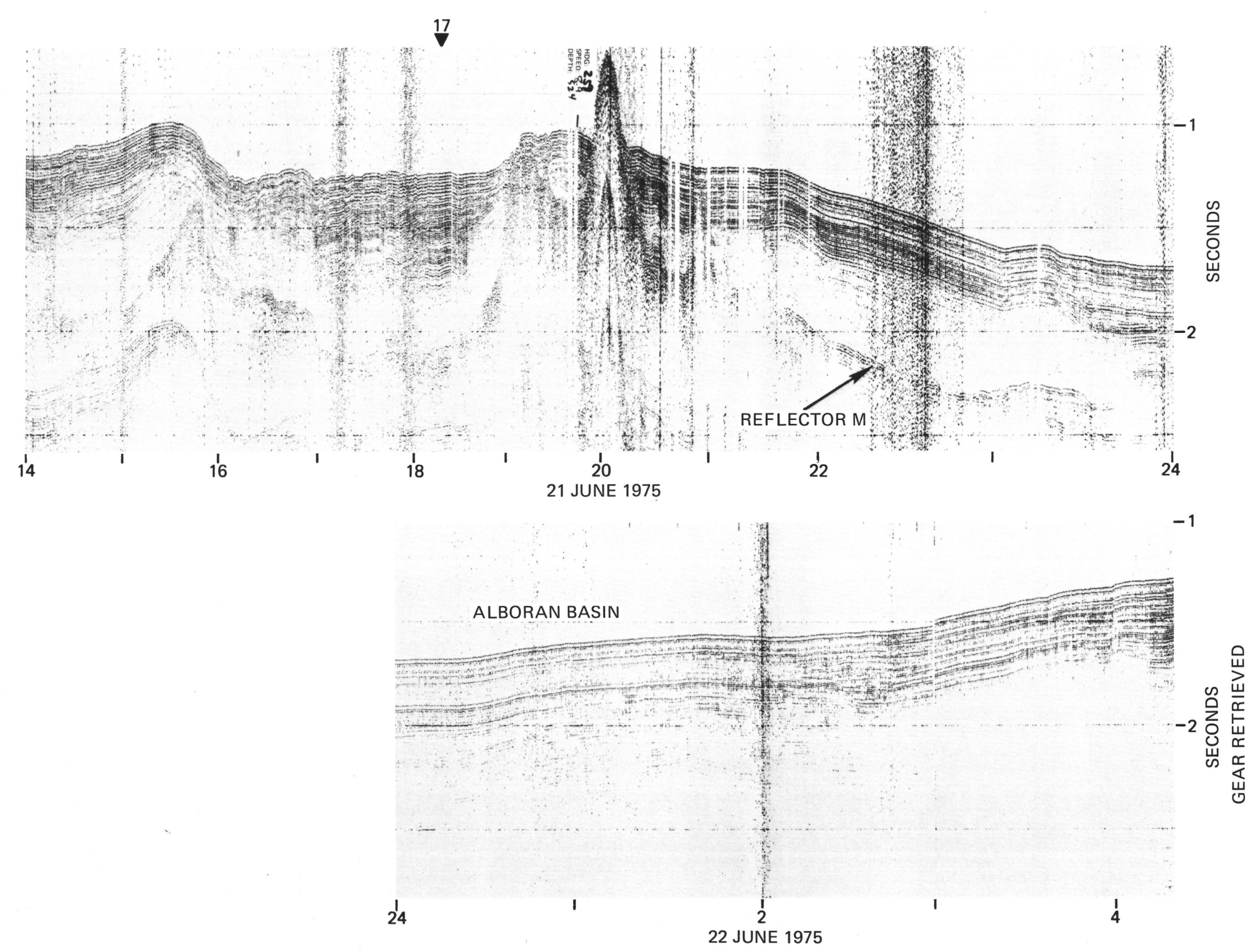

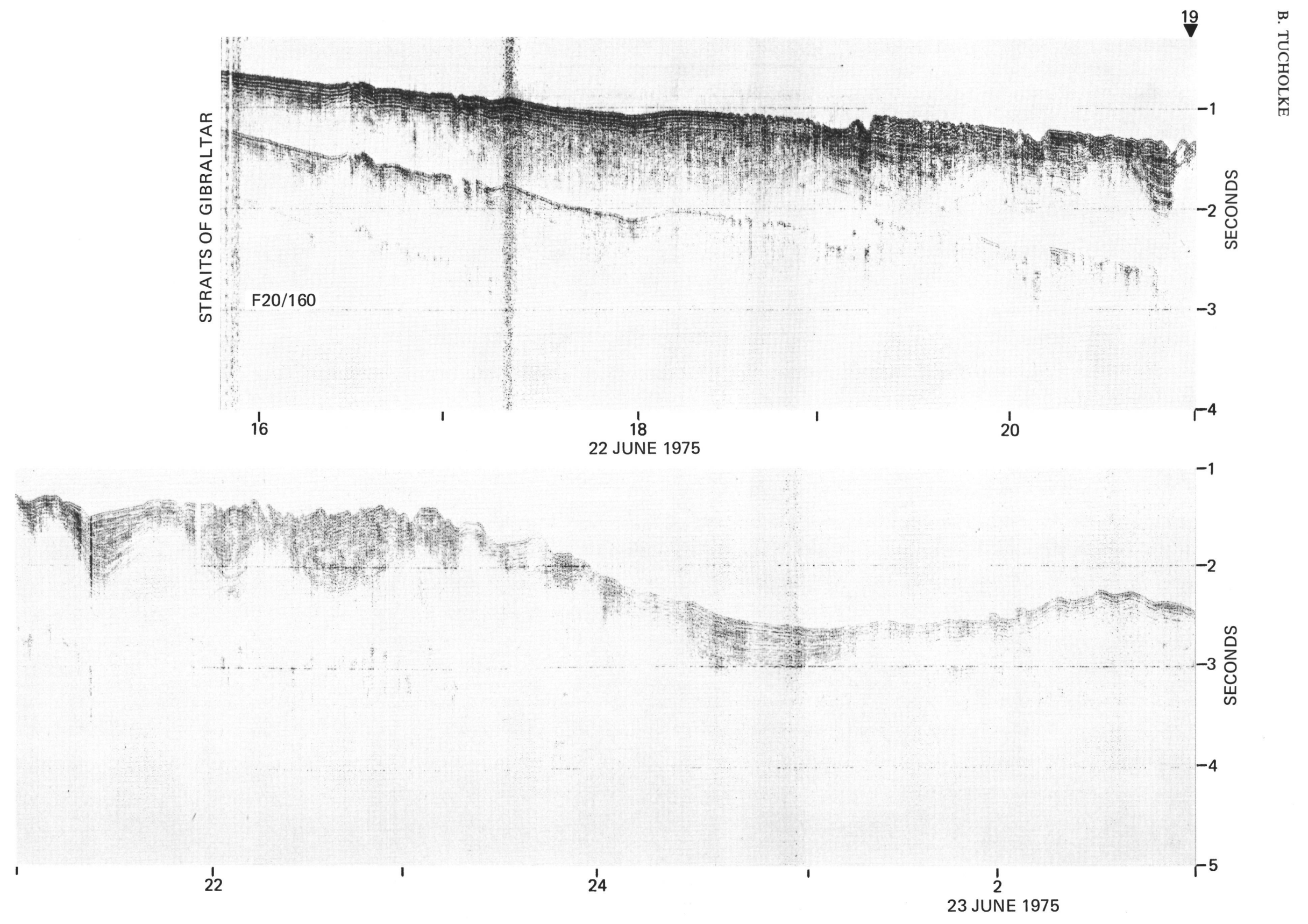

Figure 2. (Continued). 

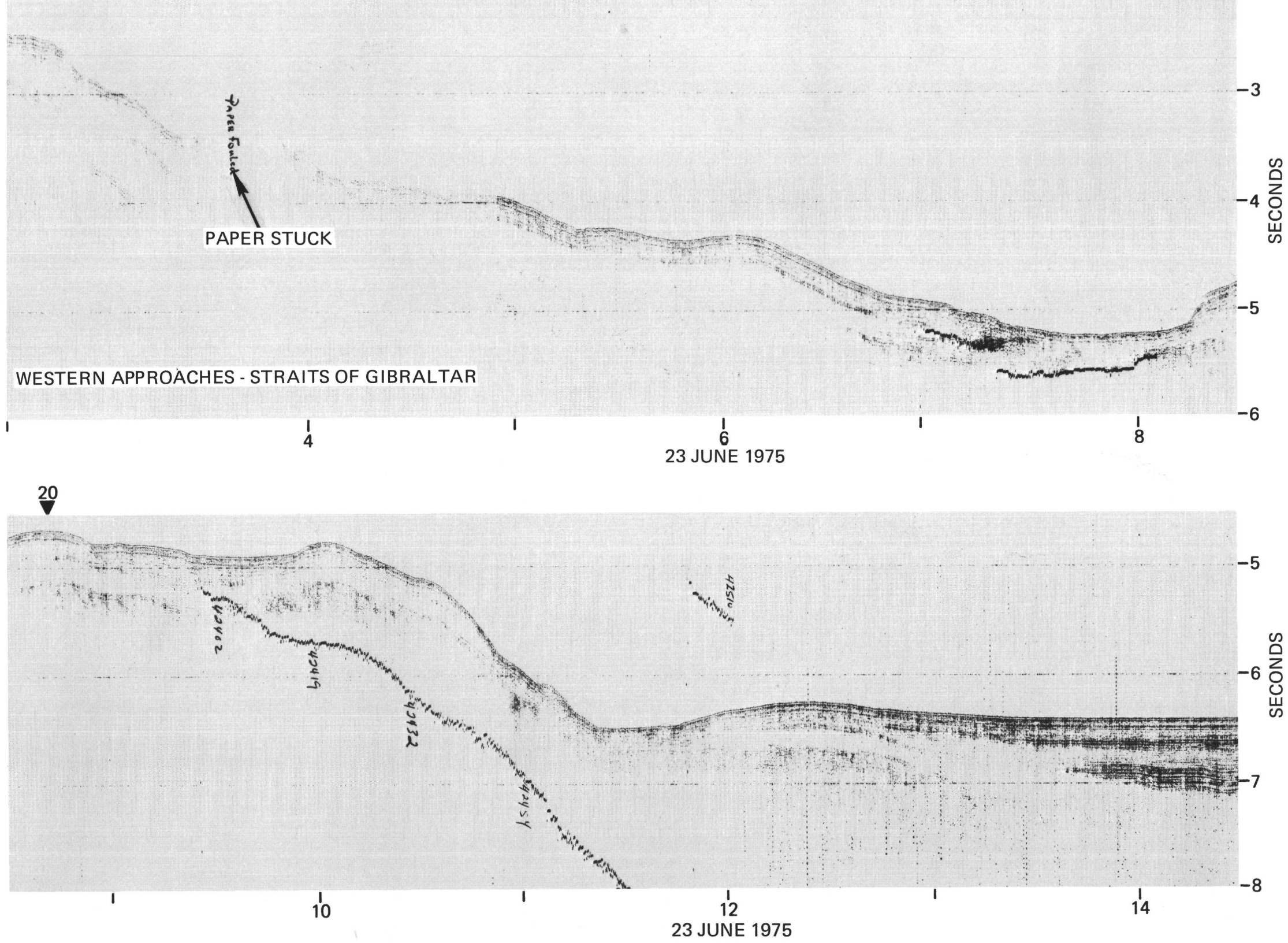

Figure 2. (Continued). 


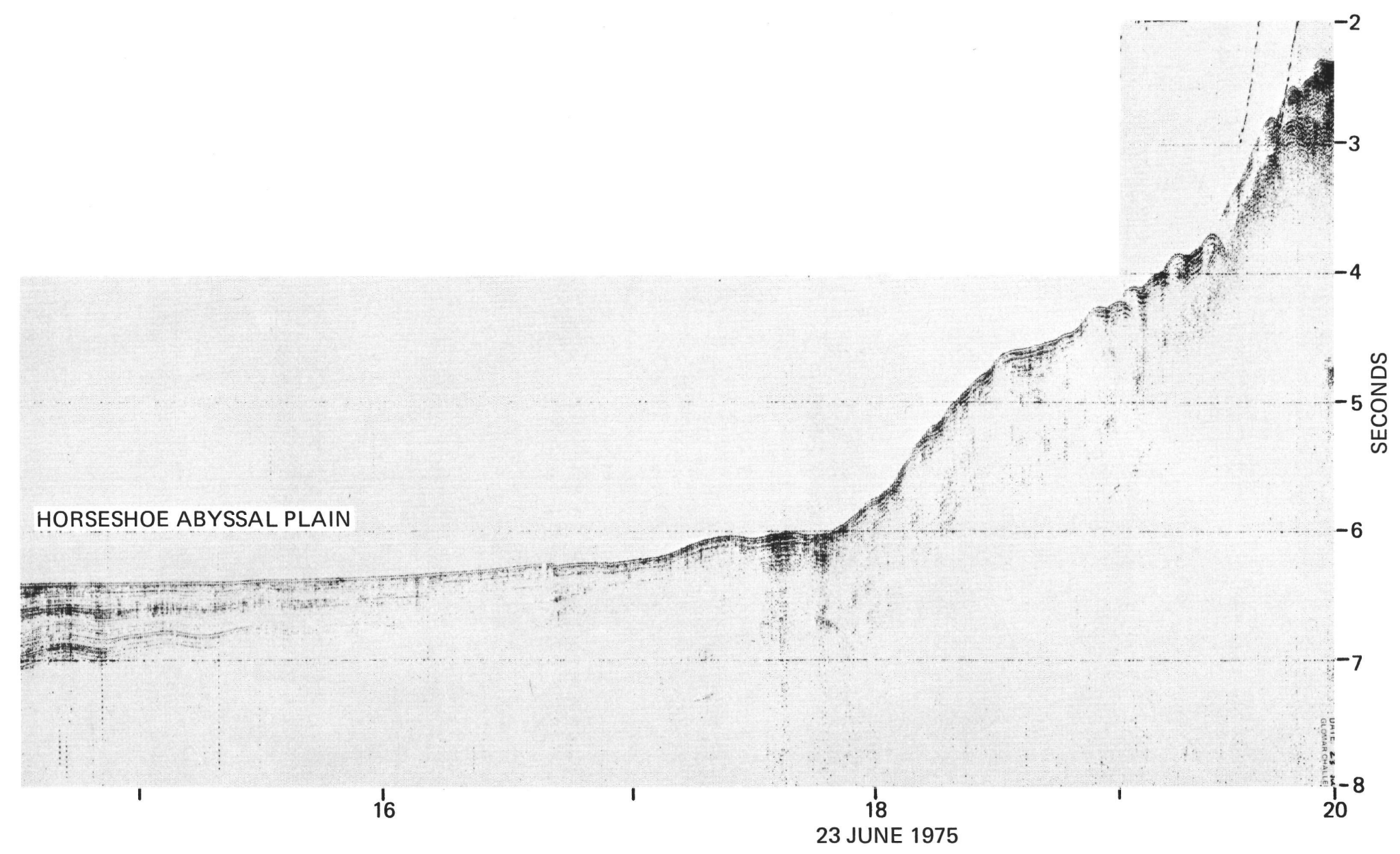

Figure 2. (Continued). 


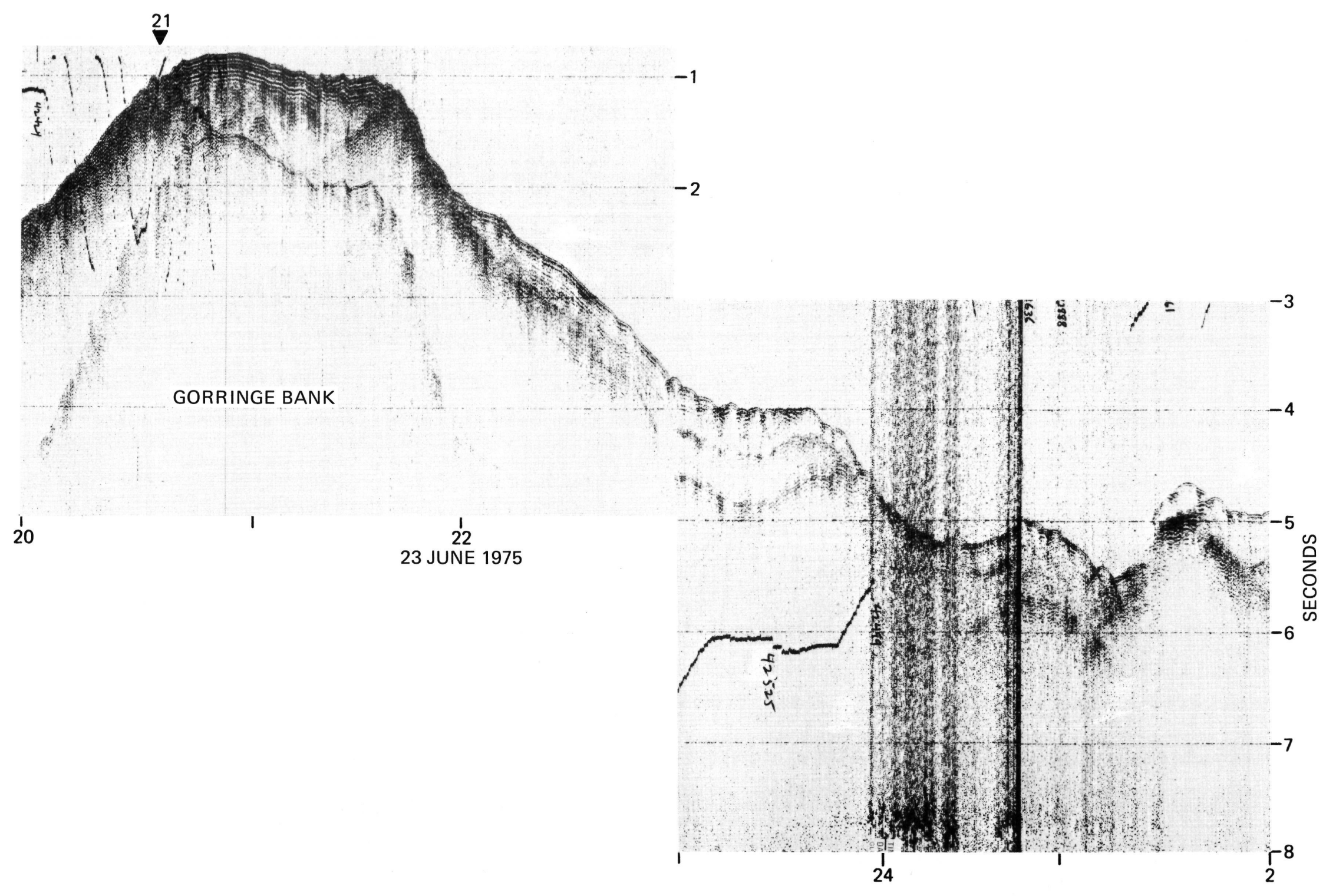

Figure 2. (Continued). 


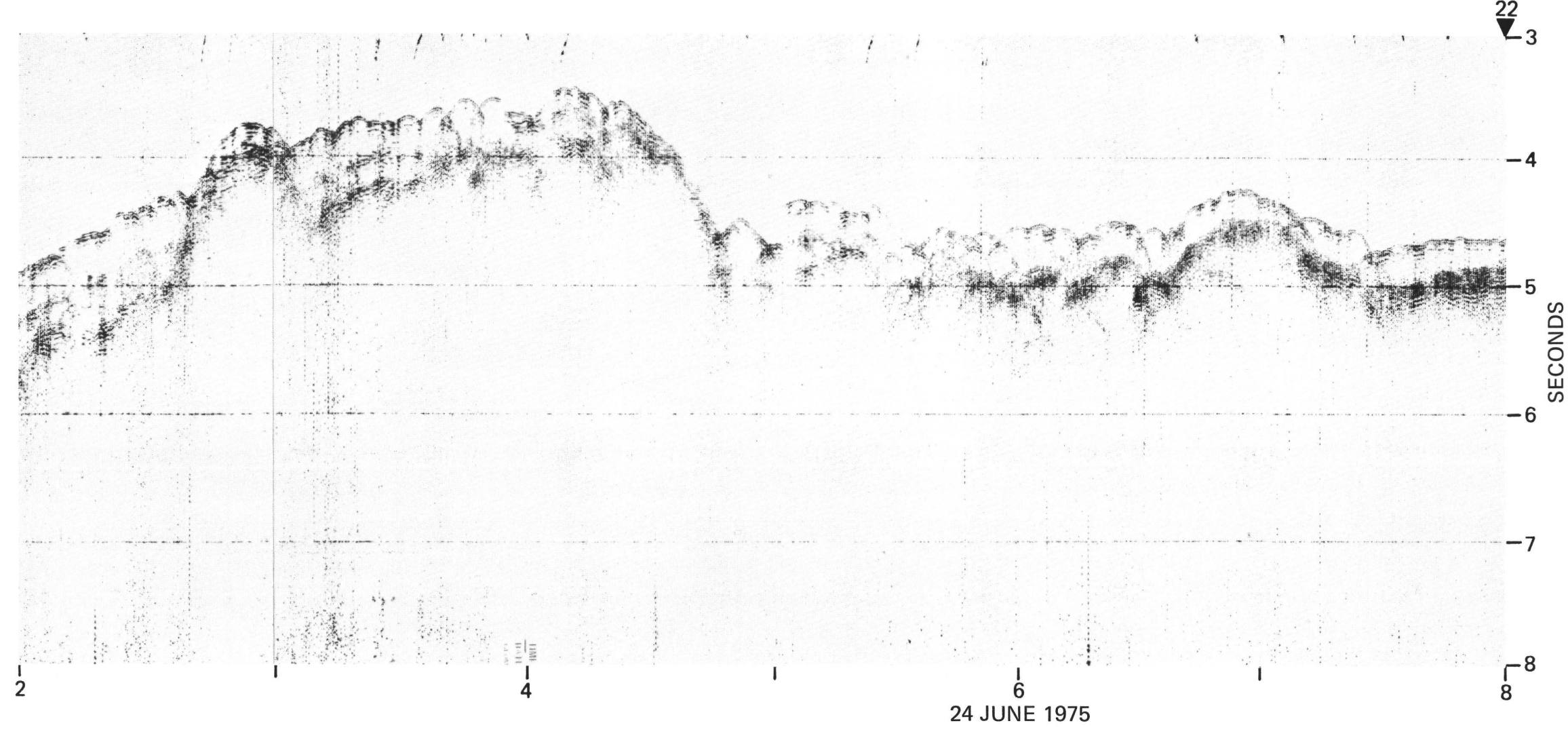

Figure 2. (Continued). 


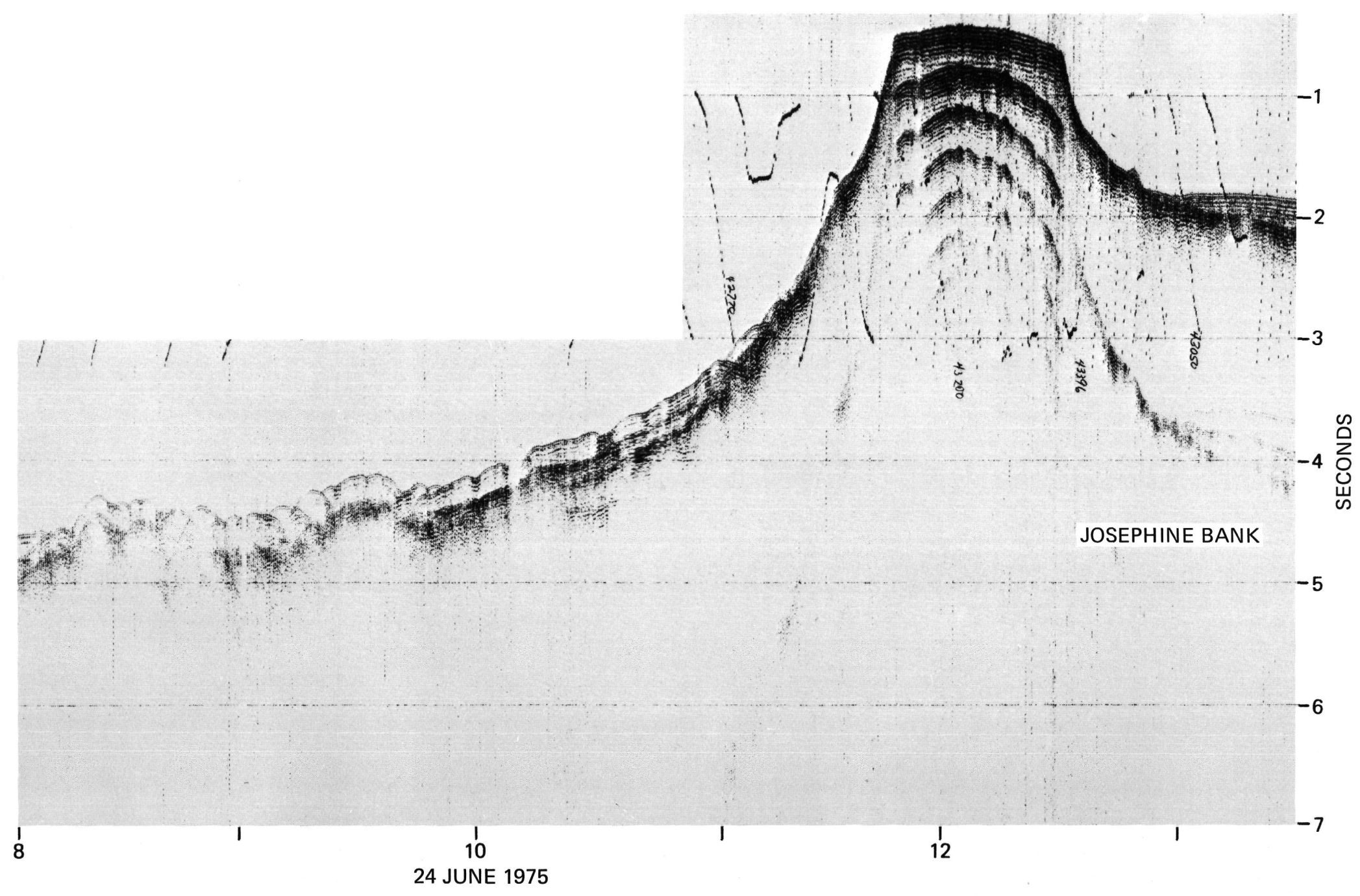

Figure 2. (Continued). 


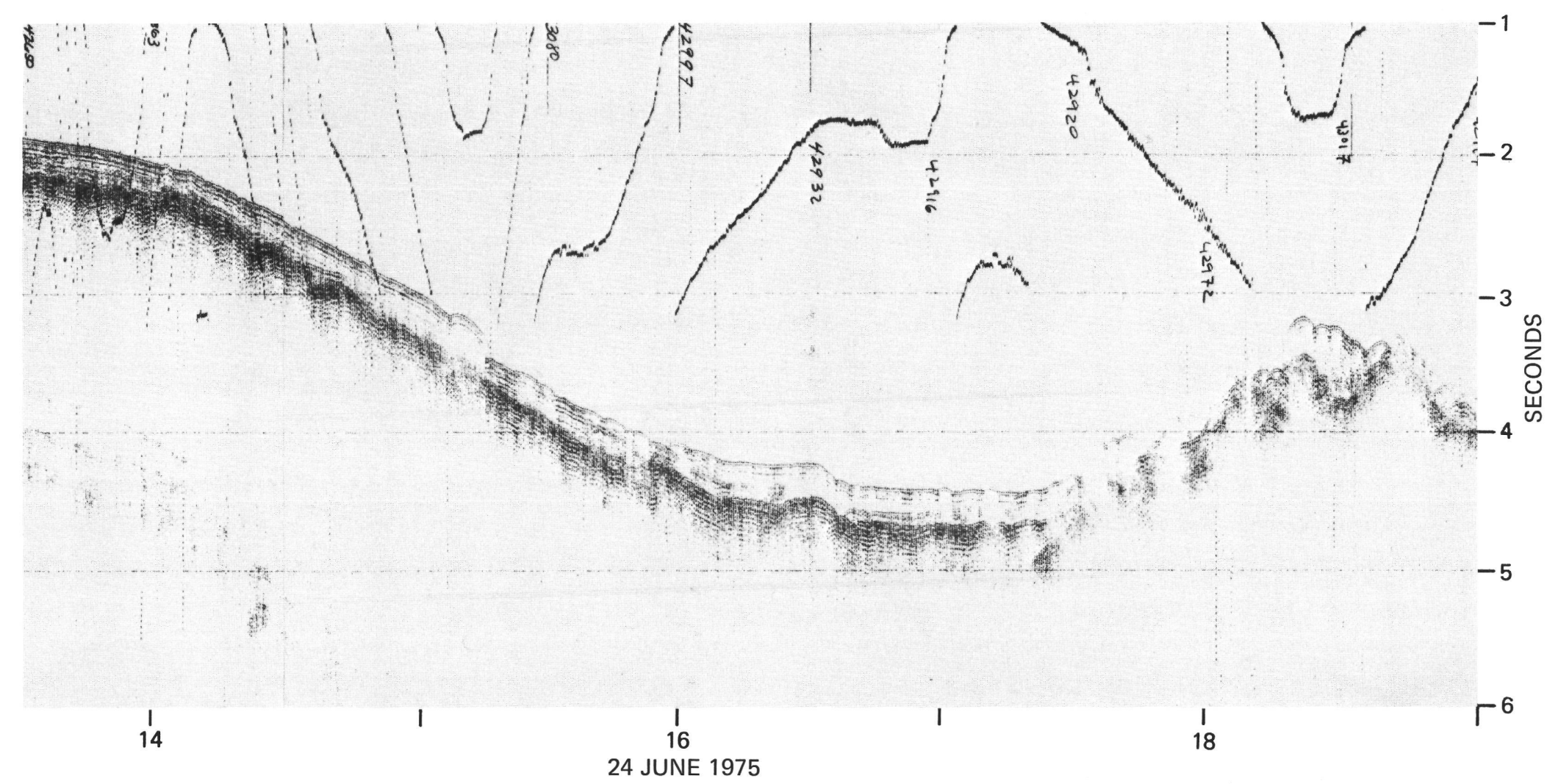

Figure 2. (Continued). 


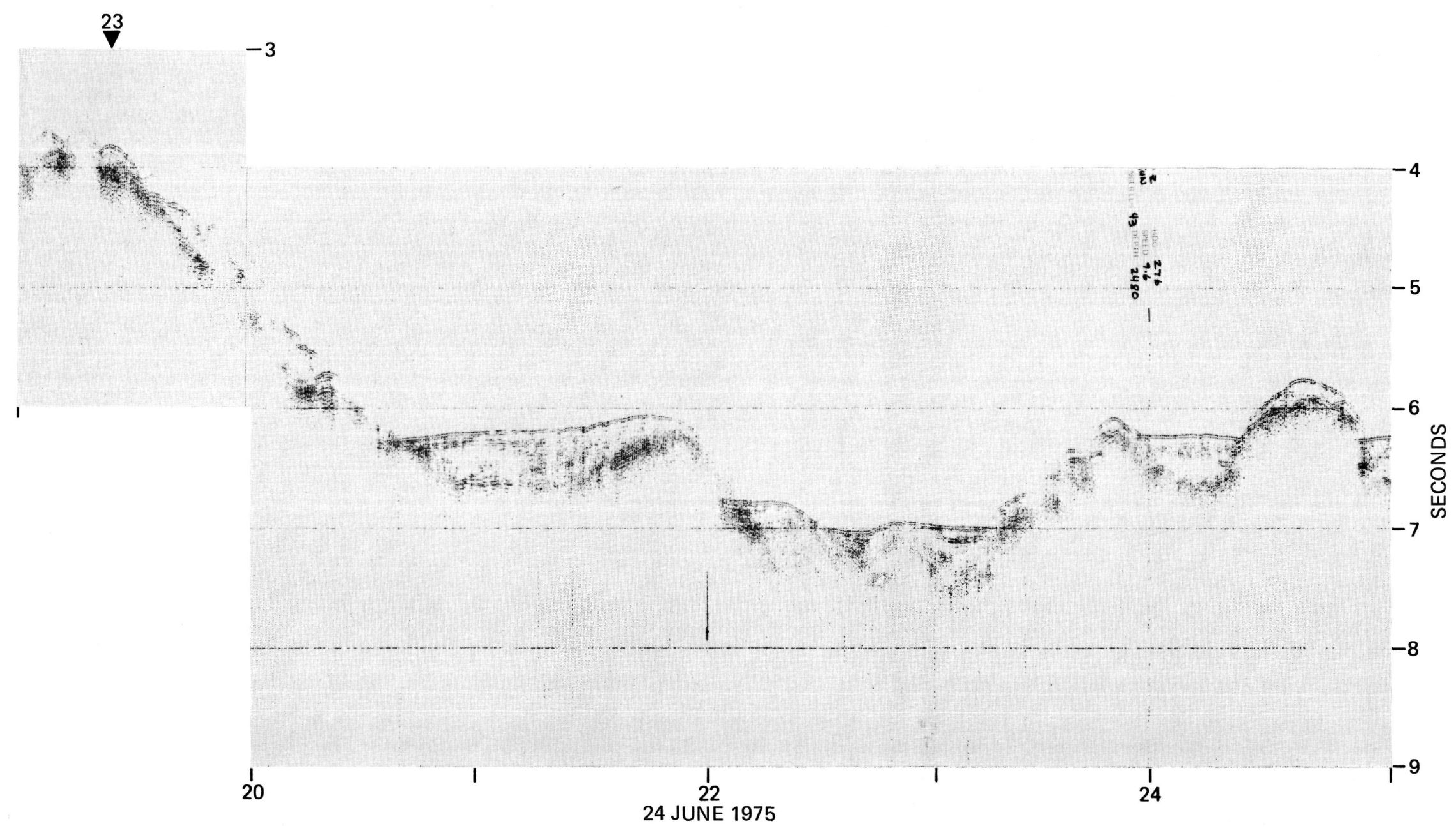

Figure 2. (Continued). 

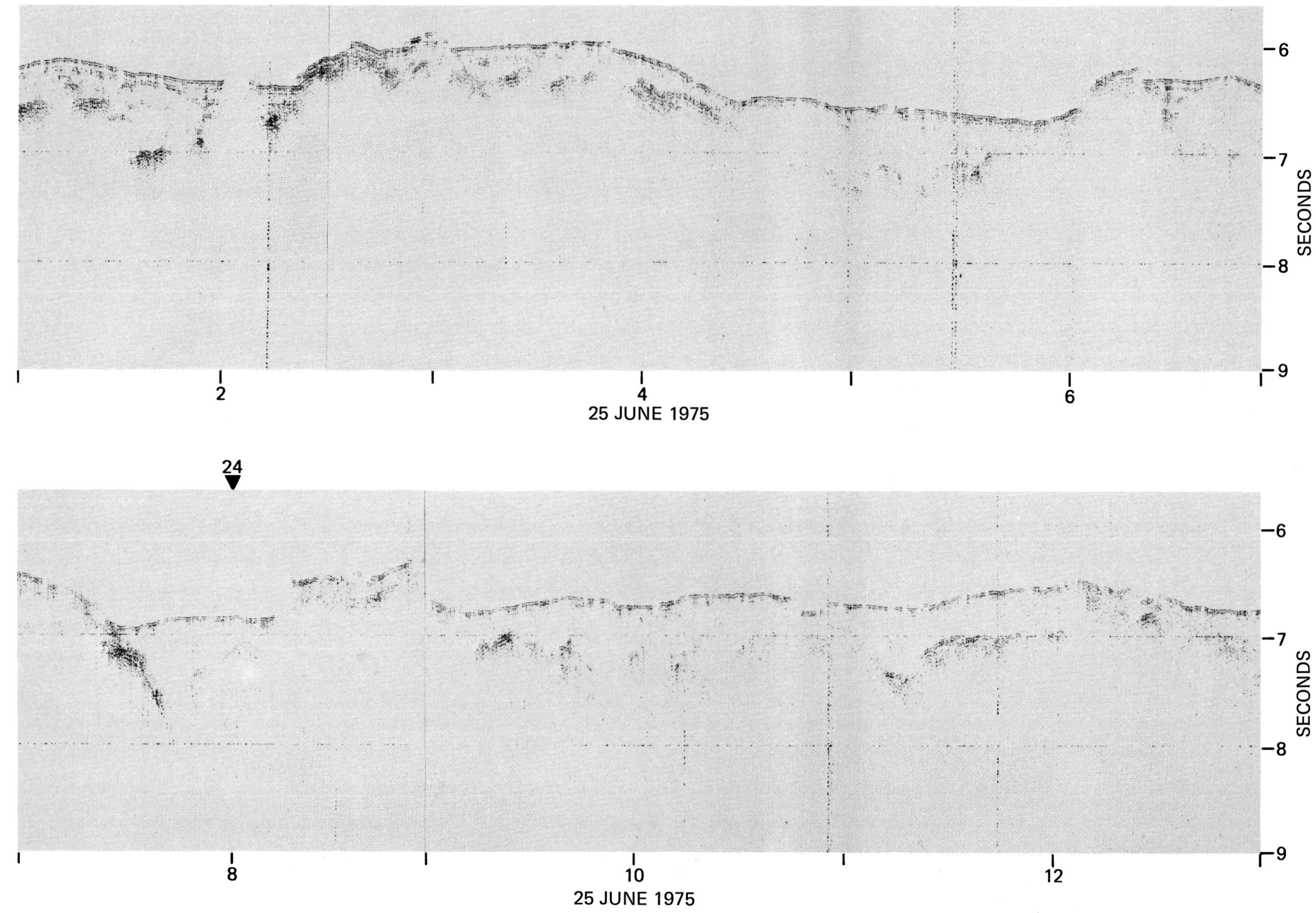

Figure 2. (Continued). 

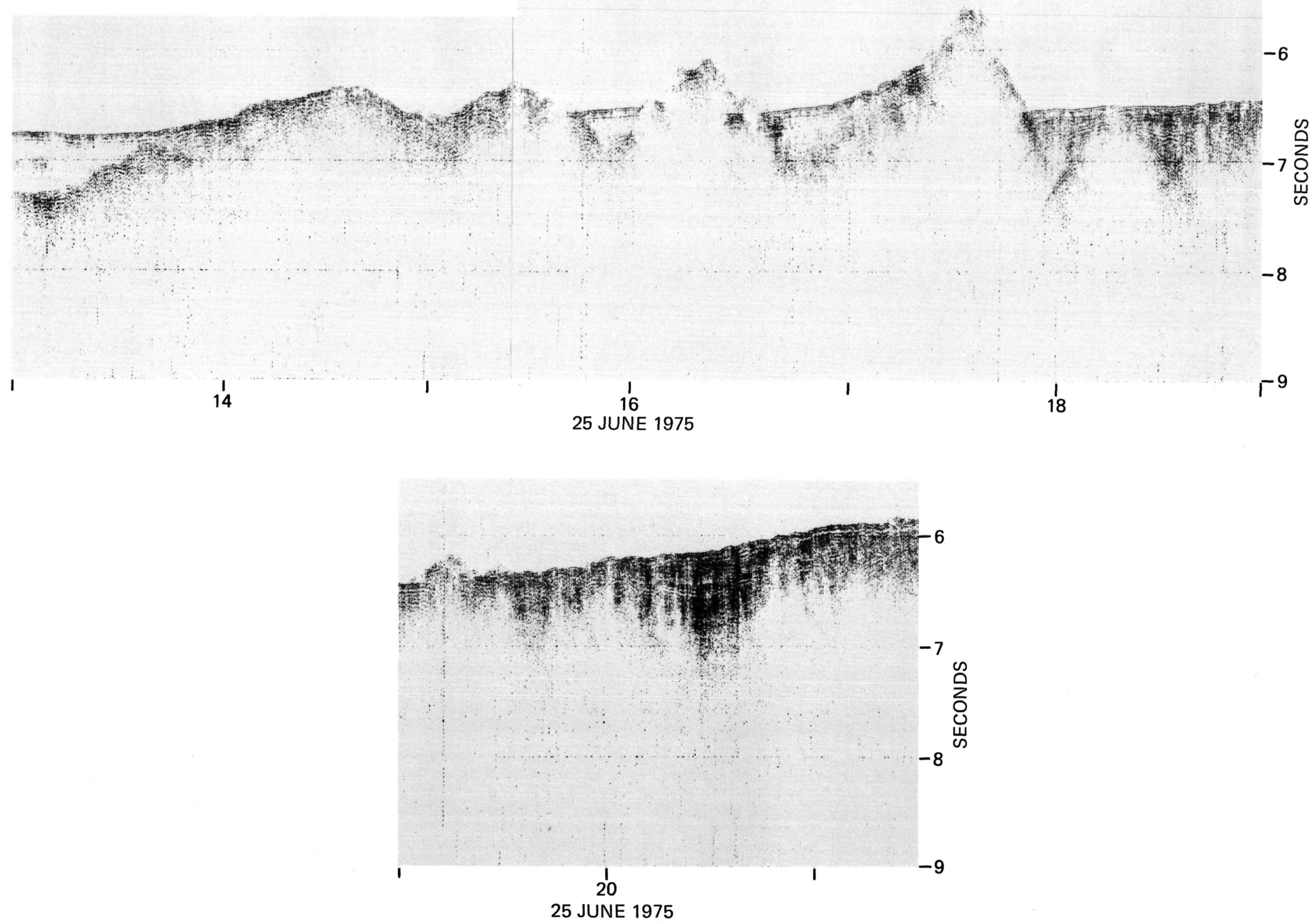

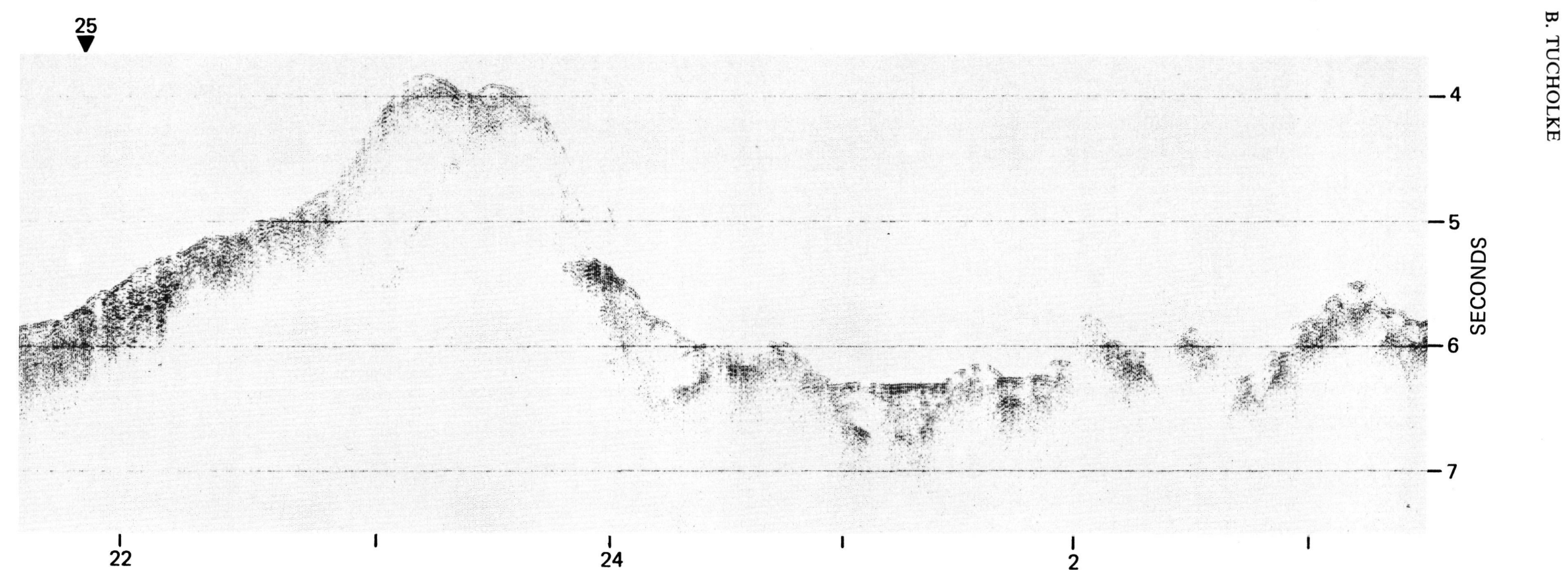

${ }_{24}^{1}$

26 JUNE 1975

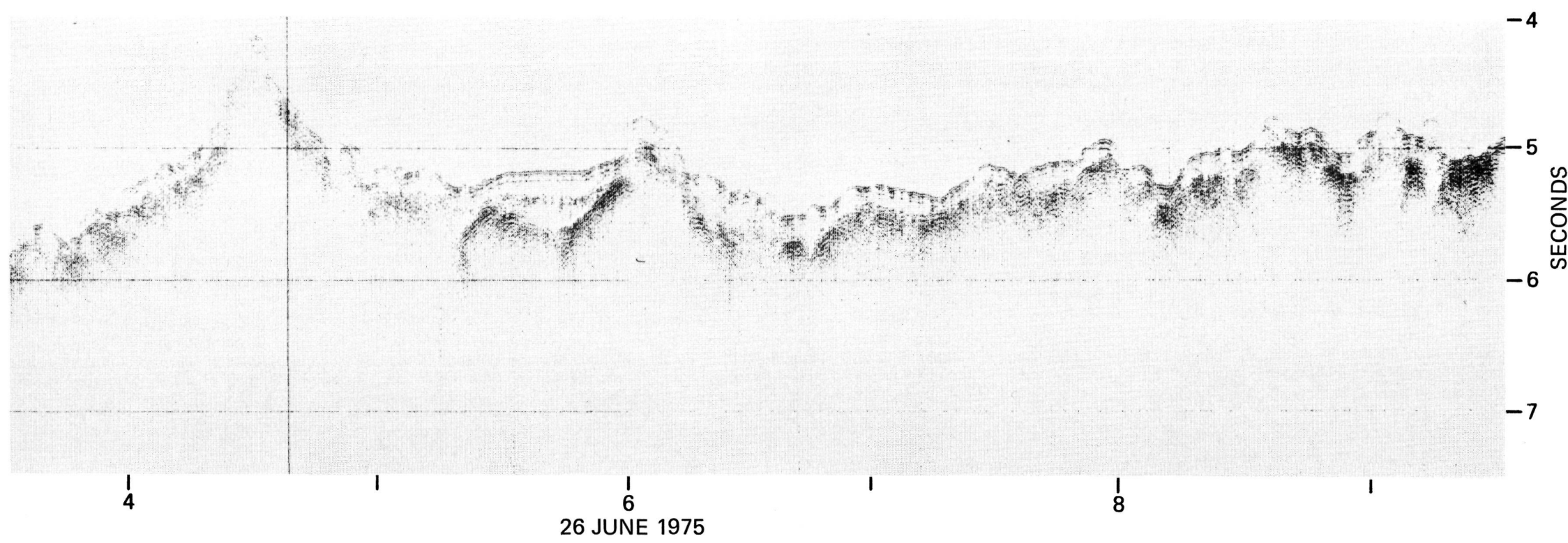

Figure 2. (Continued). 


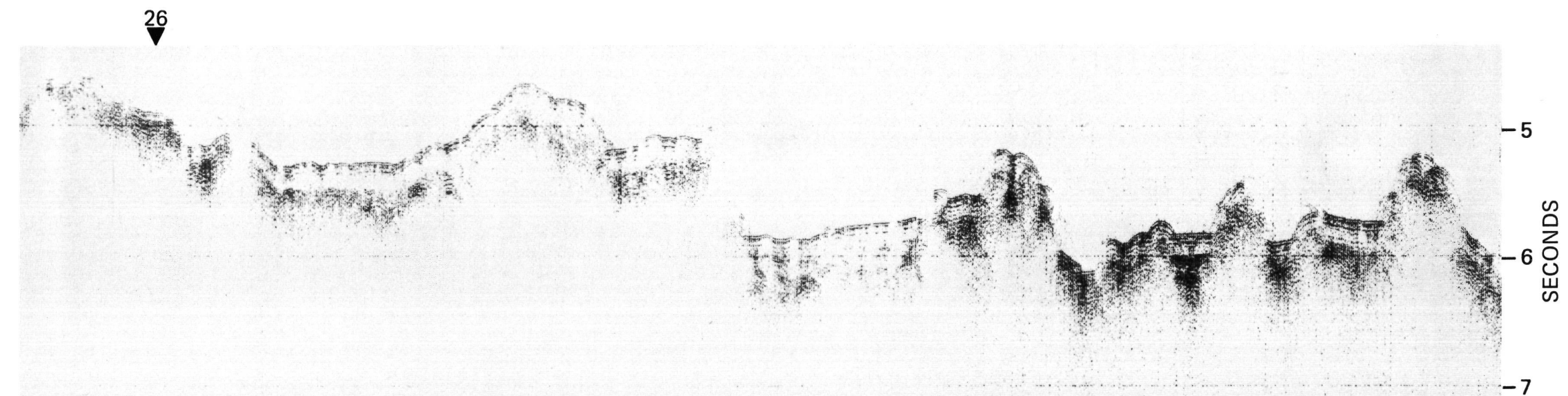

10

$\stackrel{1}{12}$

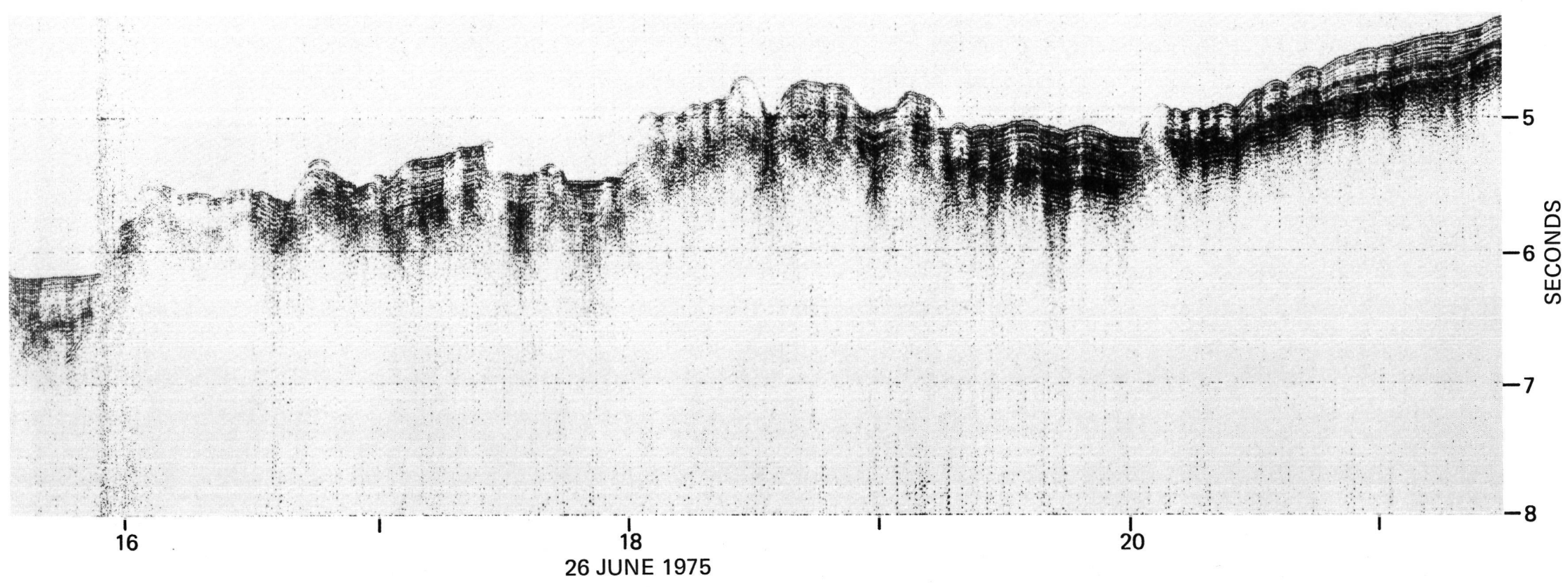




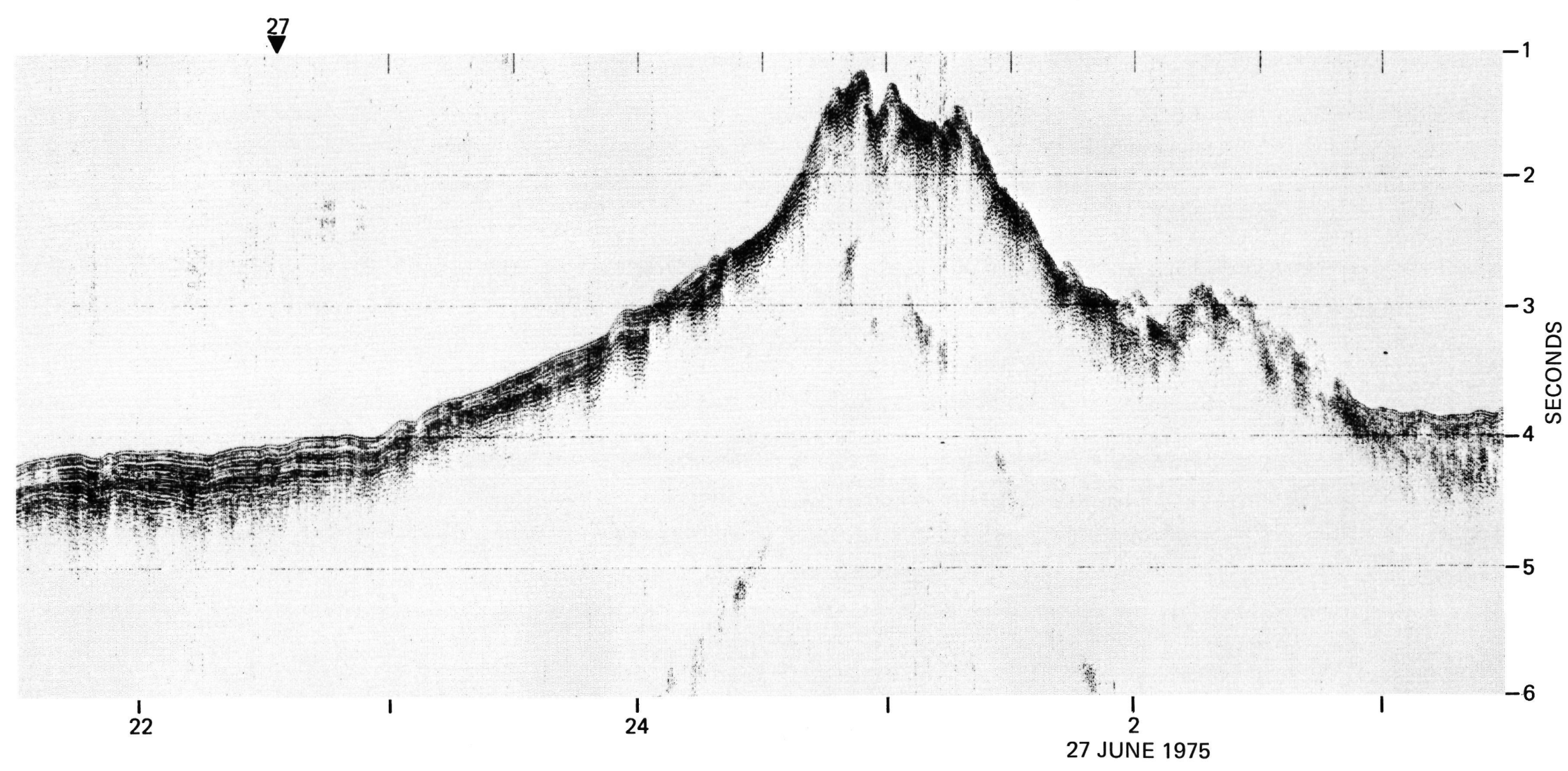

Figure 2. (Continued). 


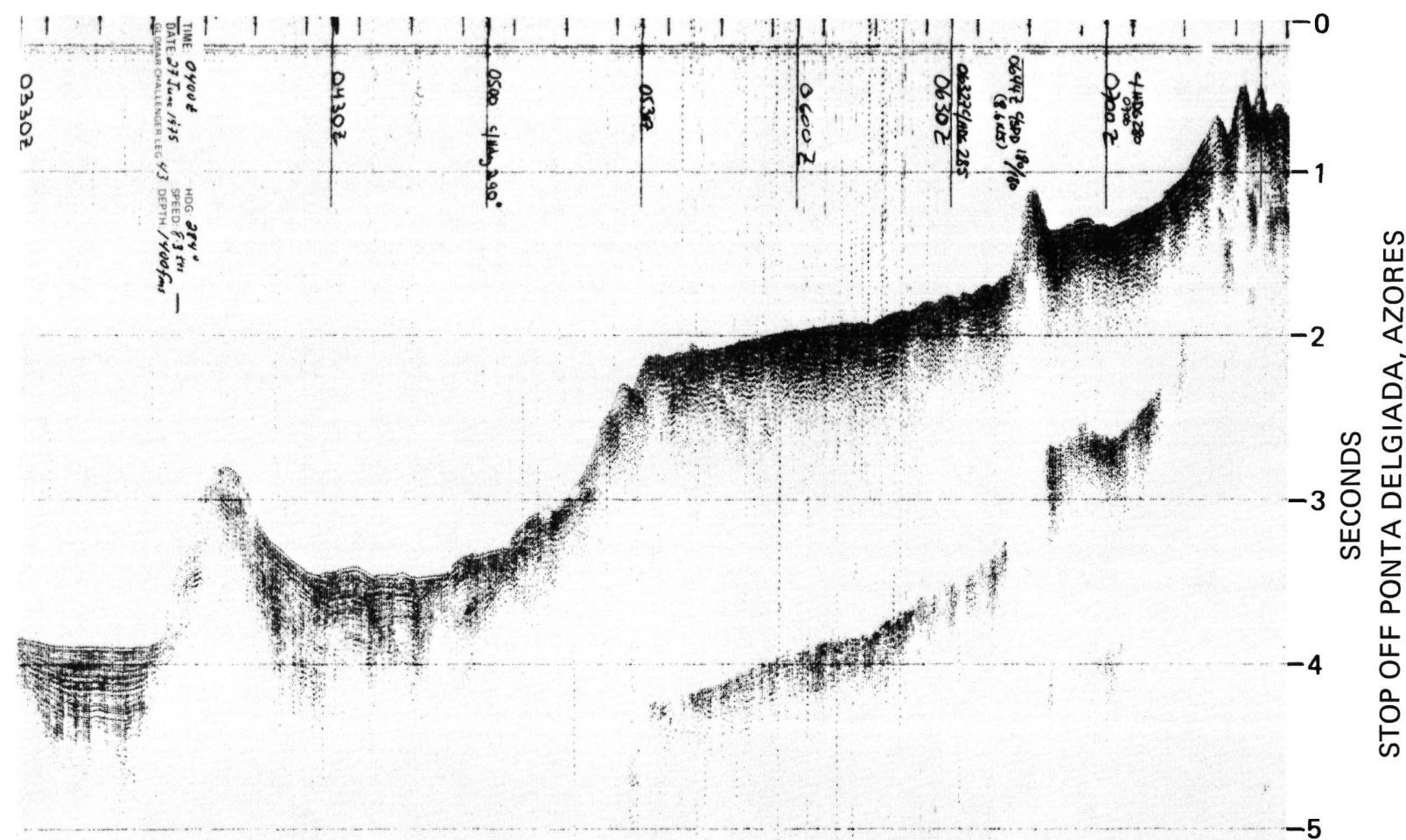

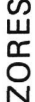

政

占

응

0
0
o
s

4

1

$-6$

27 JUNE 1975

Figure 2. (Continued). 


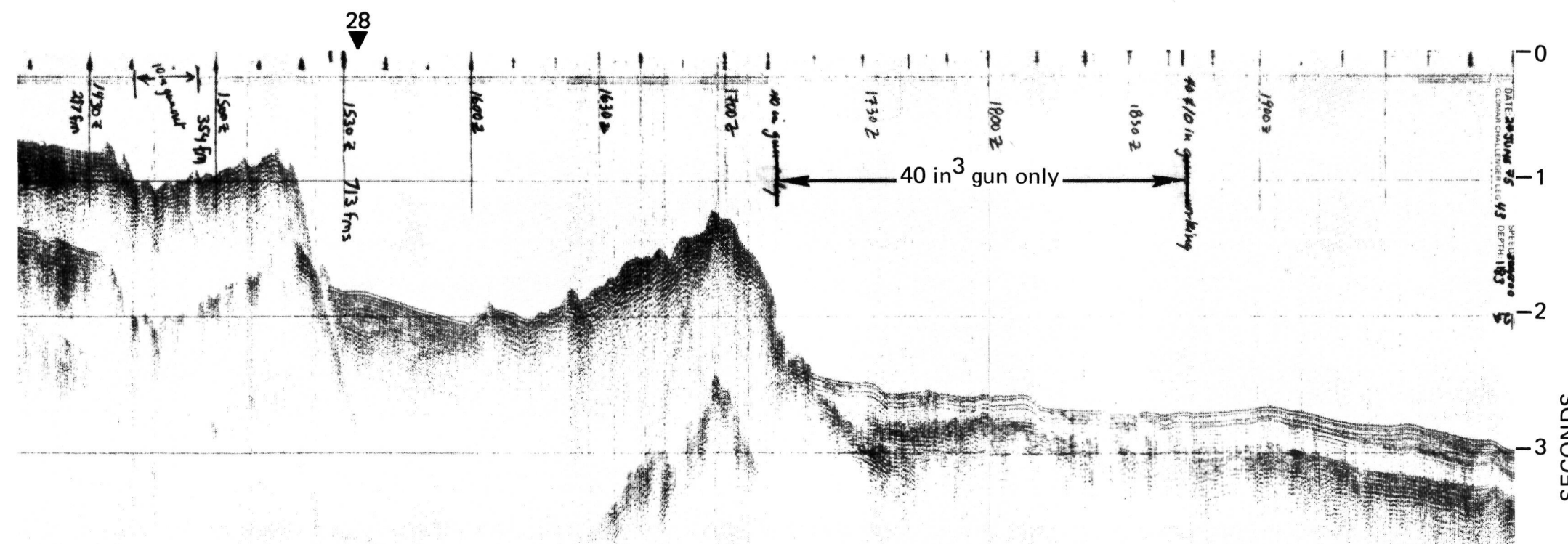

$\mathrm{F} 60 / 120$ 


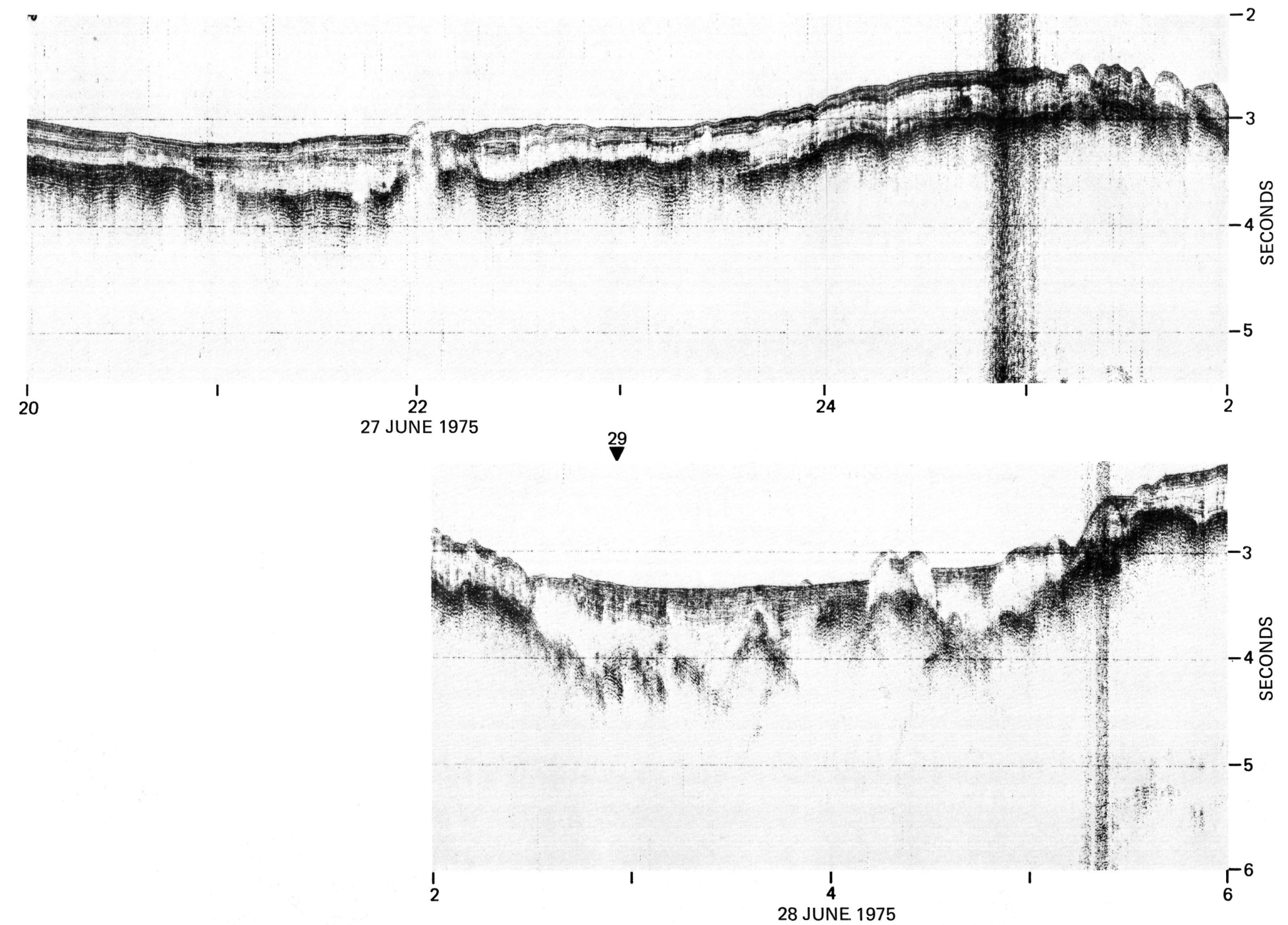




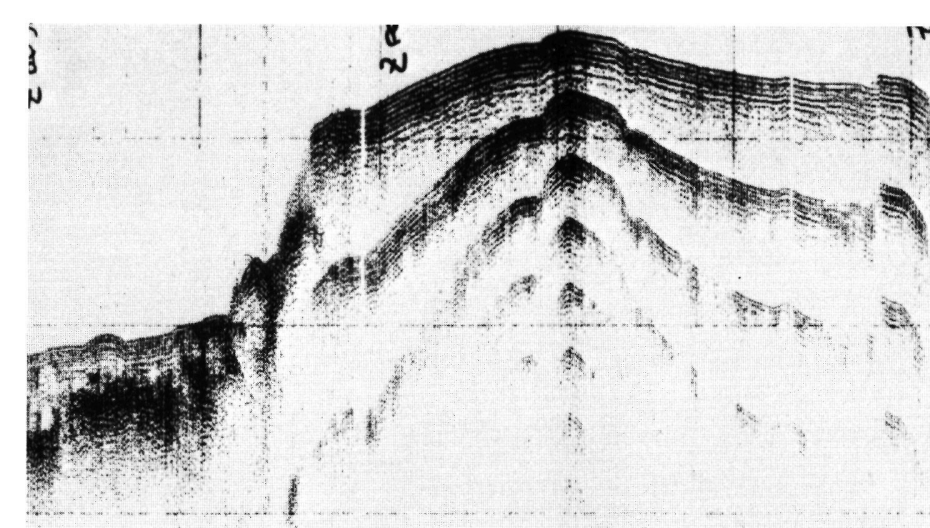

$\pi$

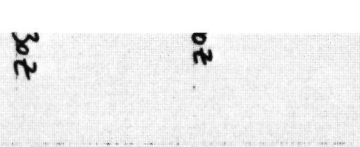

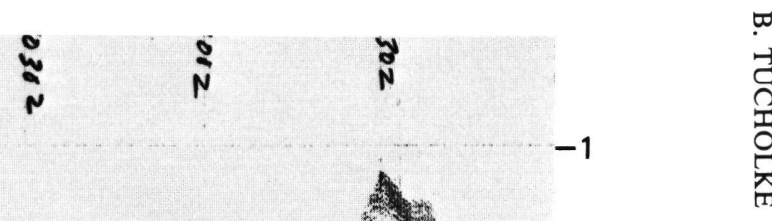

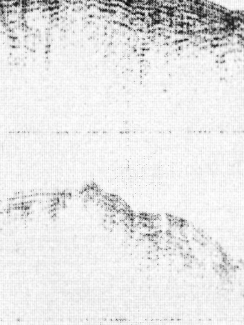

4.

-
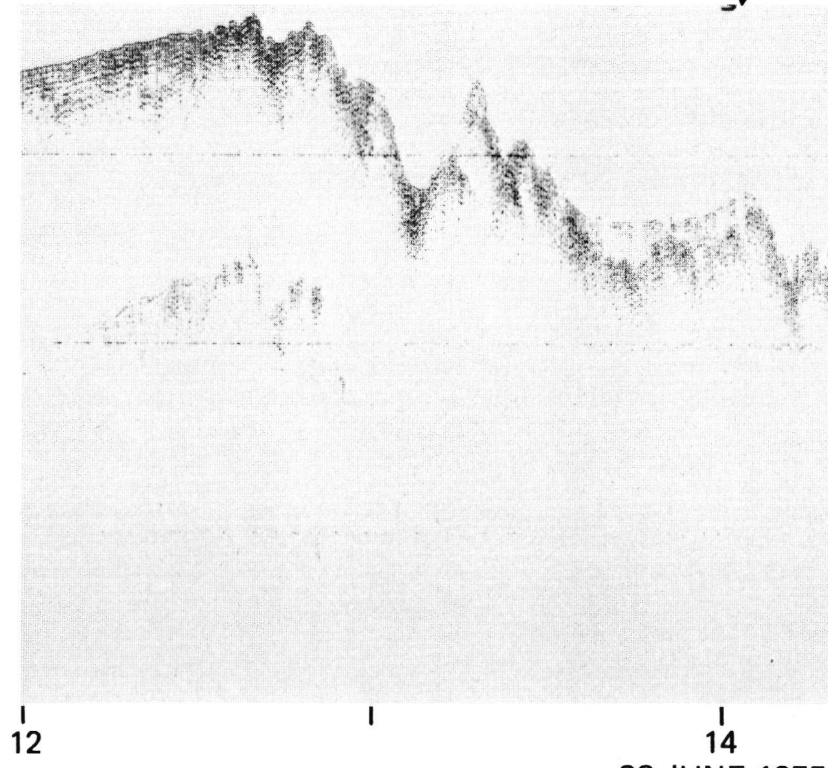

$\stackrel{3}{x}$

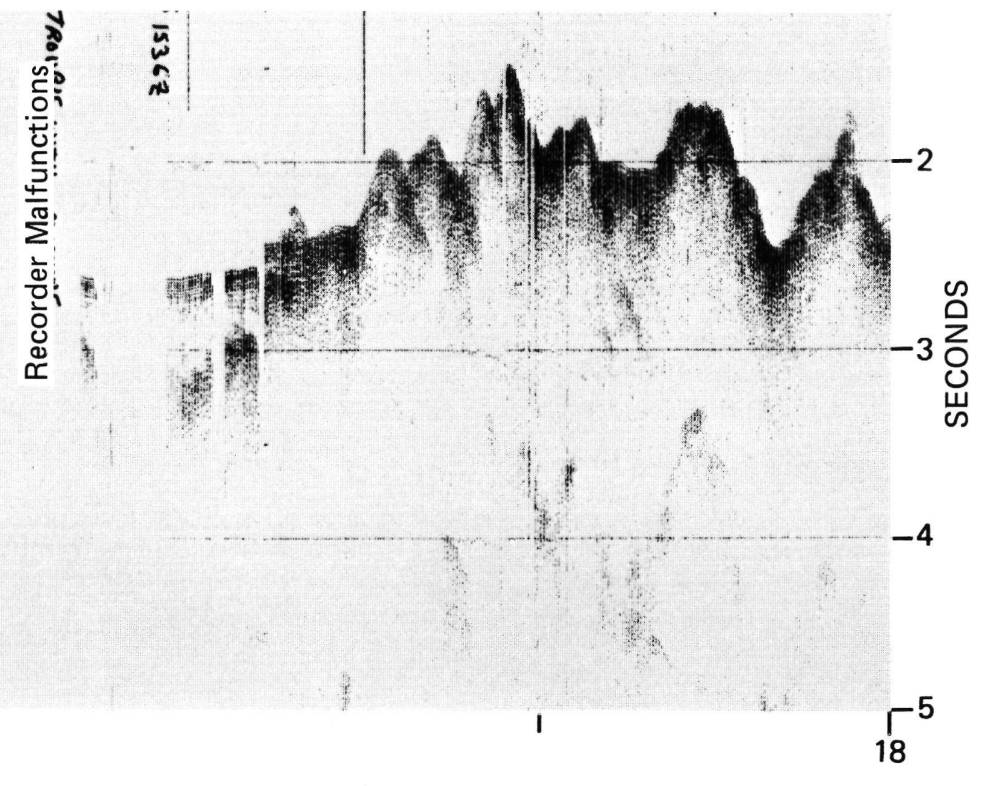

Figure 2. (Continued). 


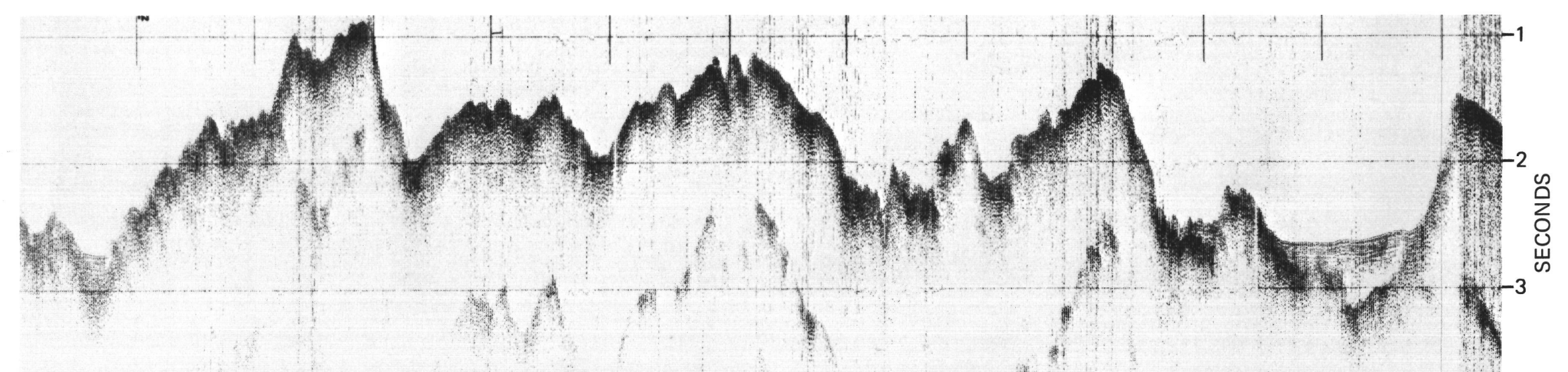

MID-ATLANTIC RIDGE CREST

$\stackrel{1}{18}$

20
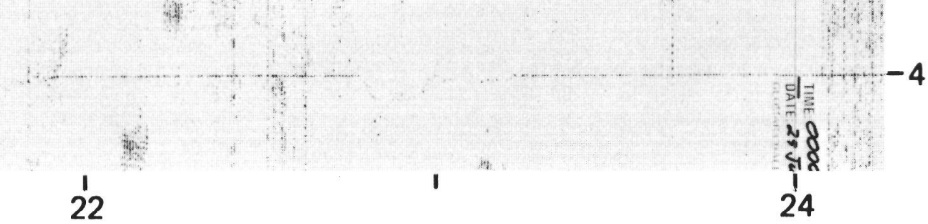

28 JUNE 1975

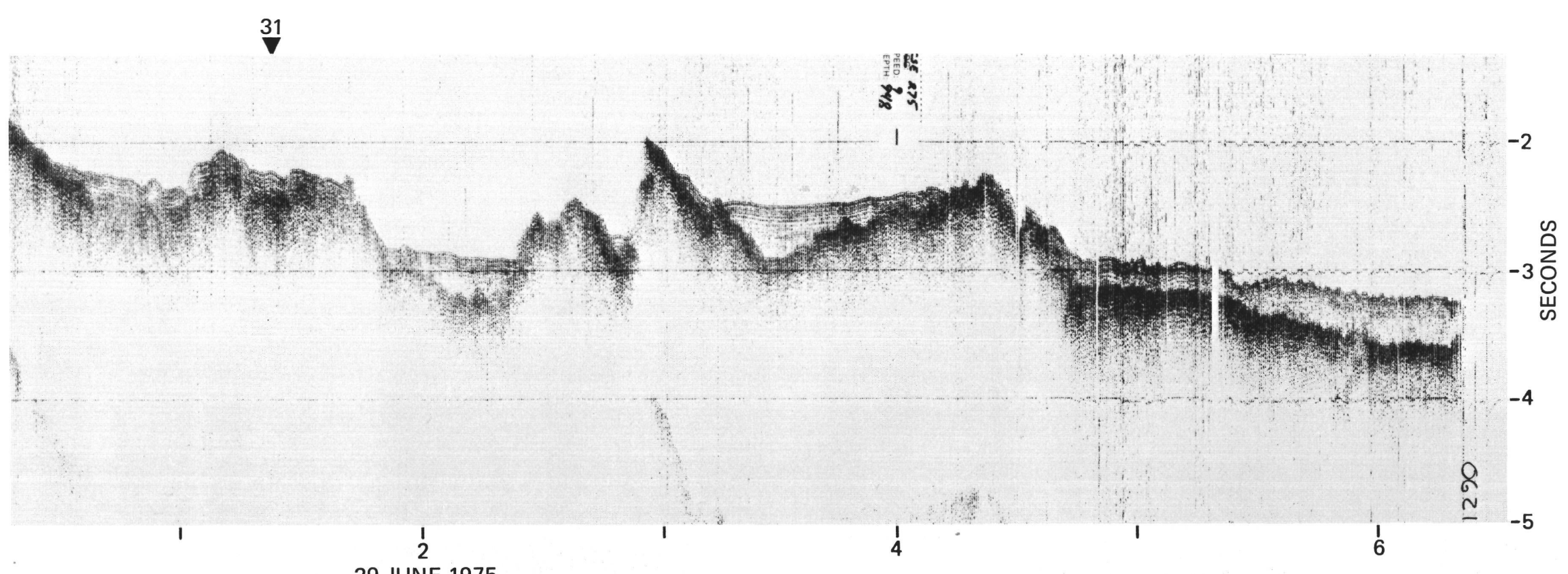

29 JUNE 1975 

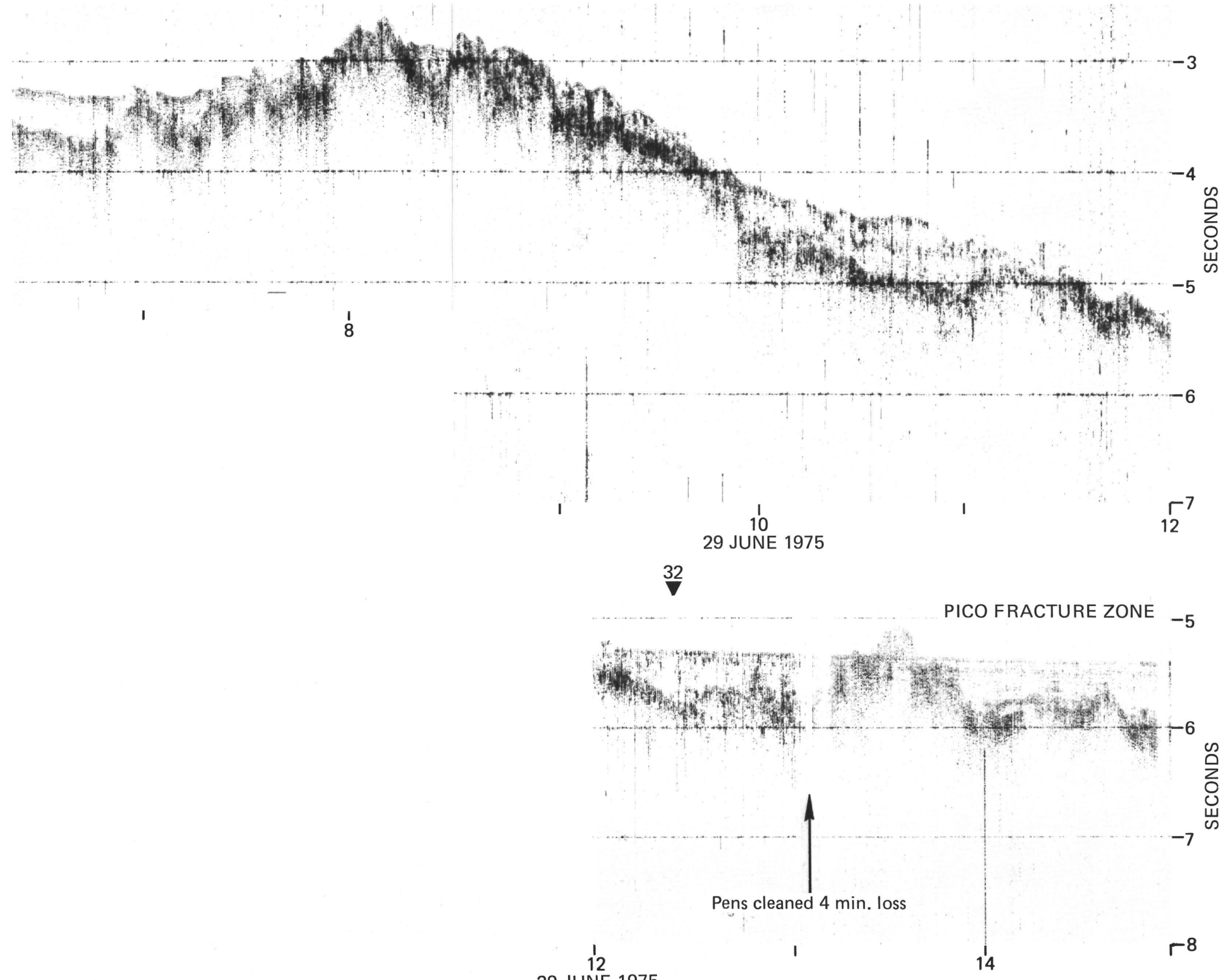

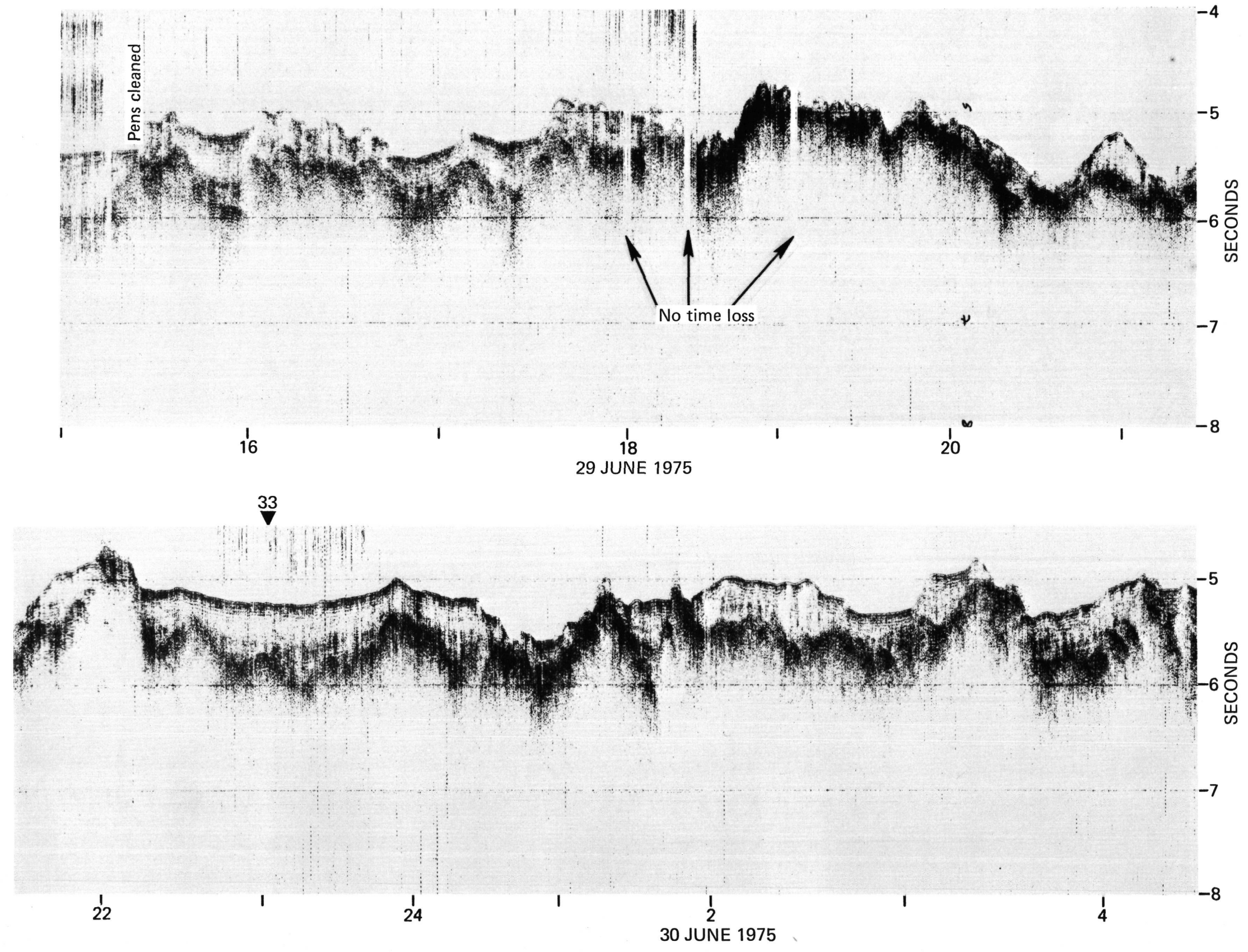


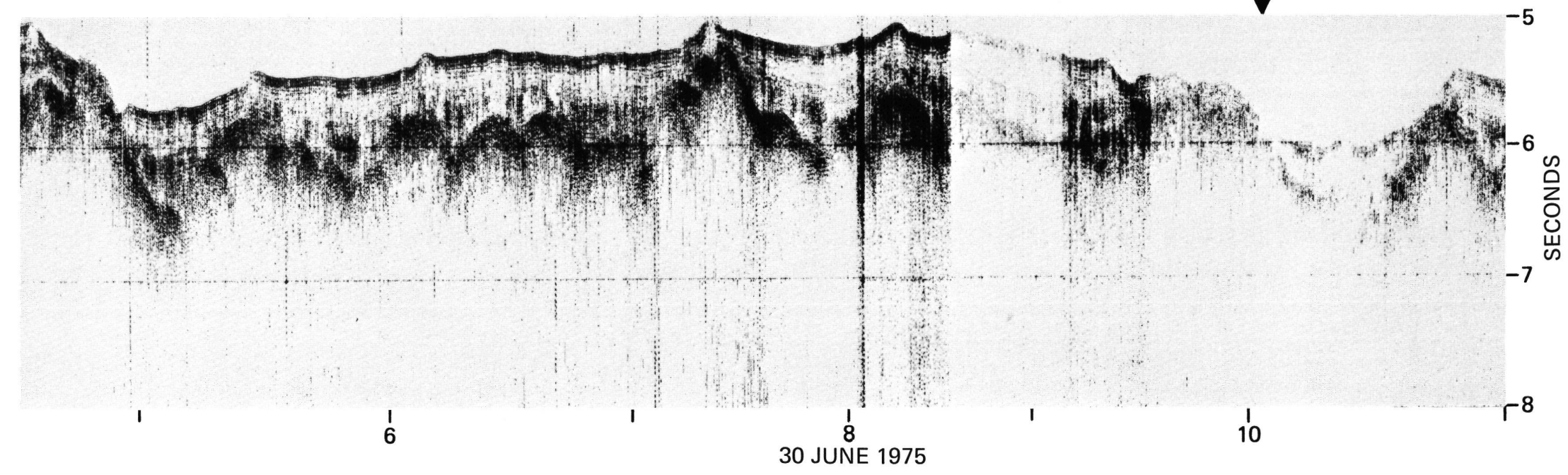

$-5$

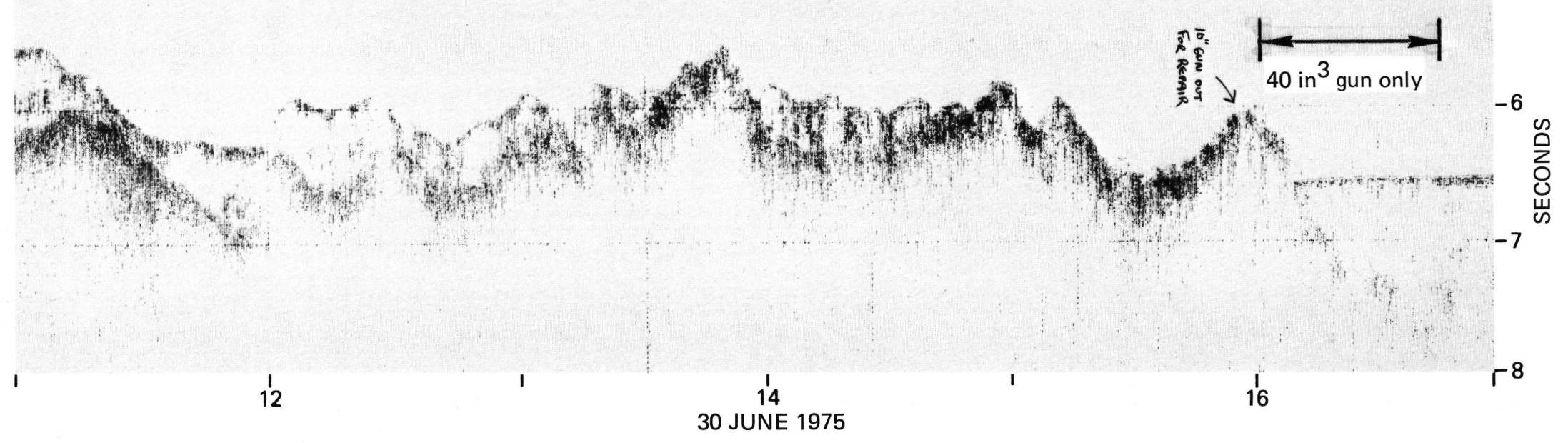

Figure 2. (Continued). 

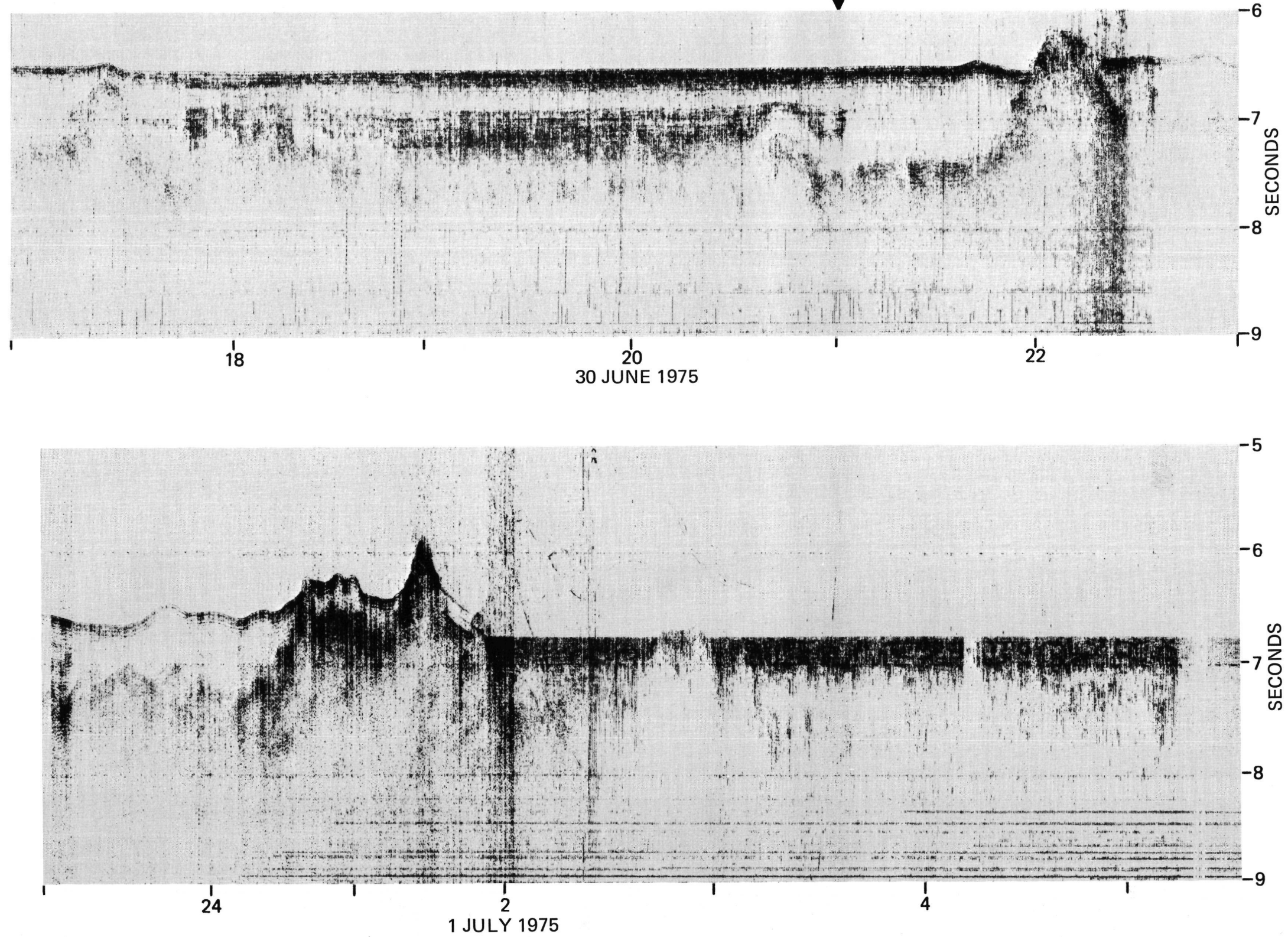

Figure 2. (Continued). 

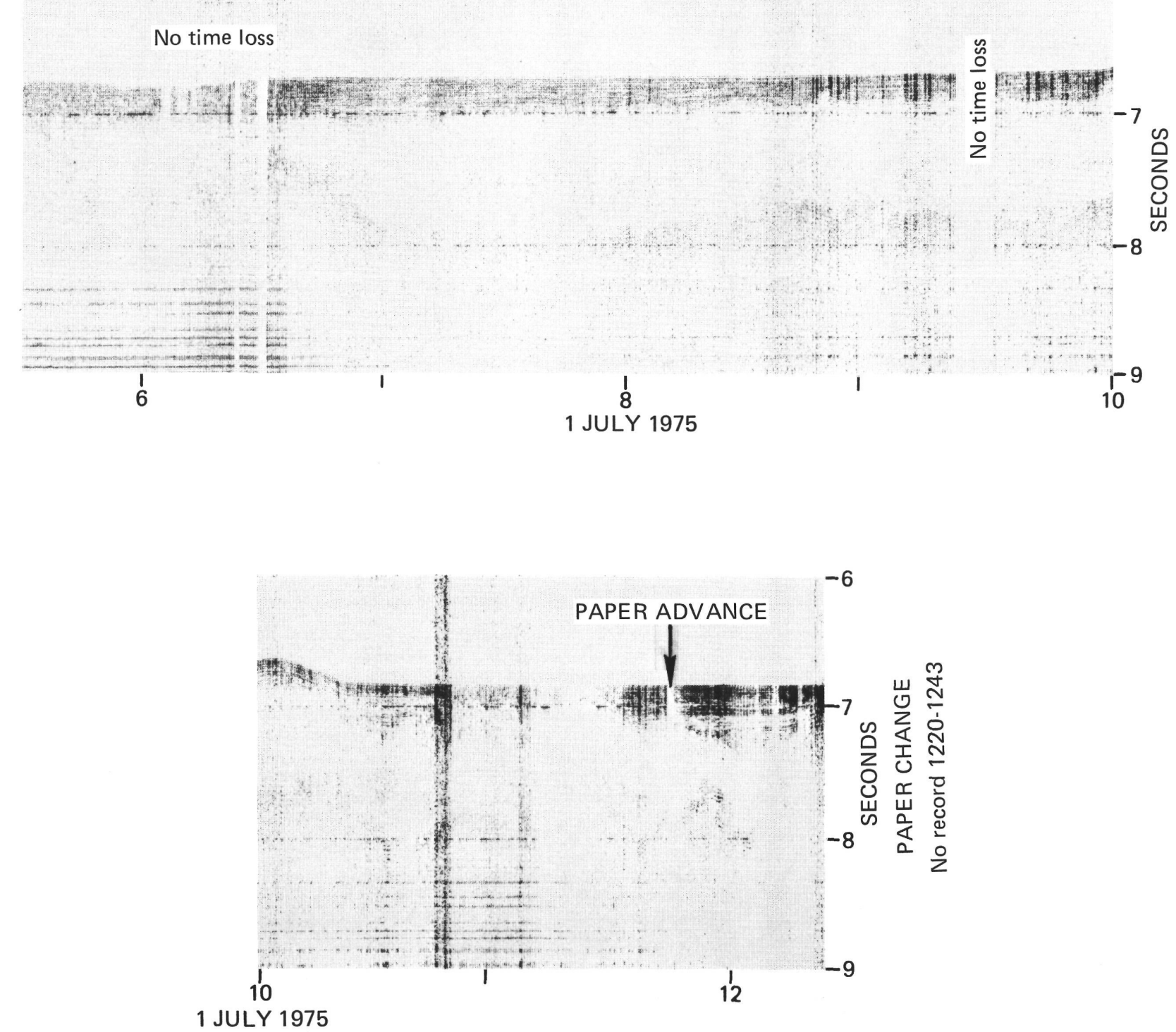

Figure 2. (Continued). 

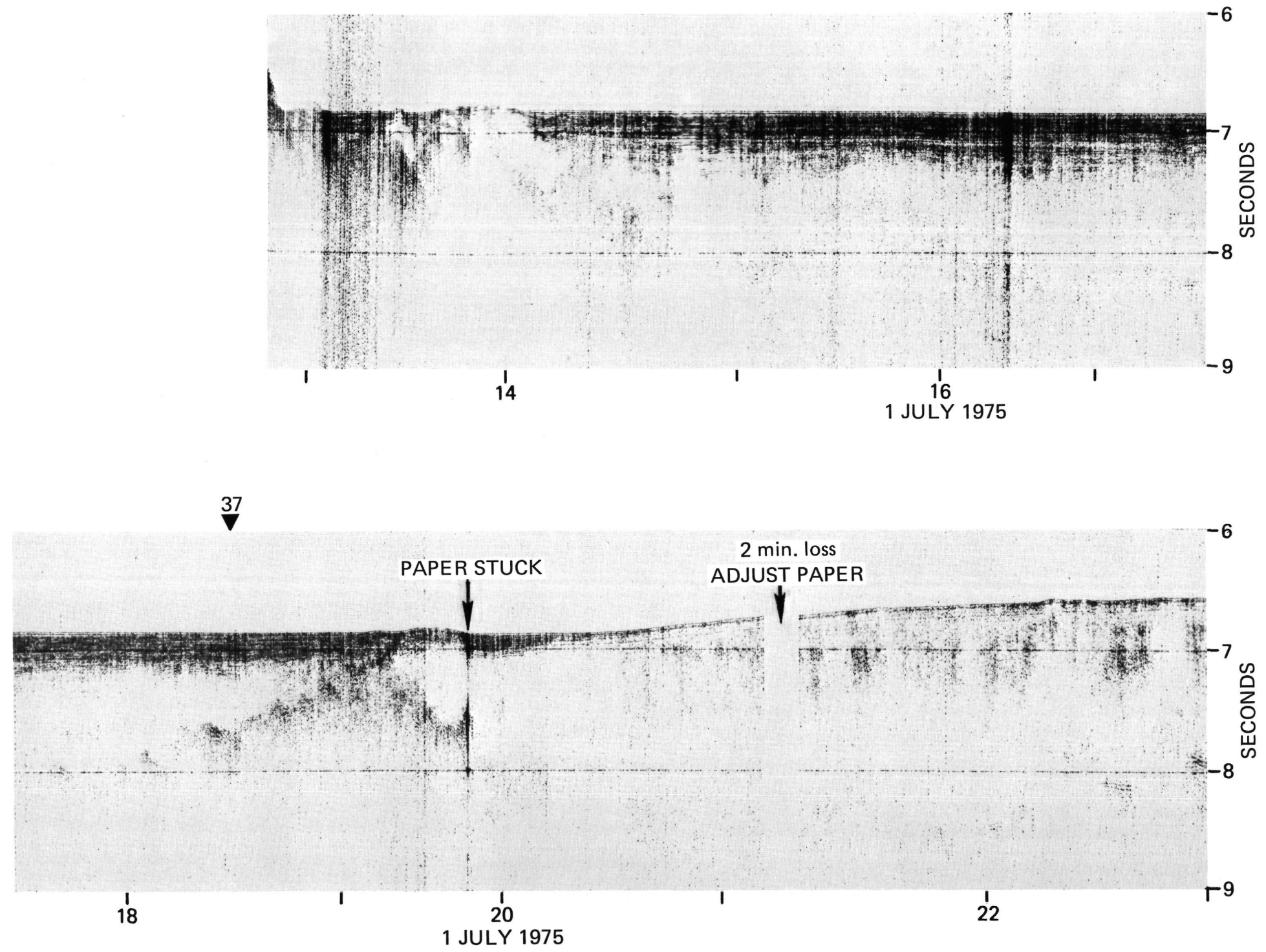

Figure 2. (Continued). 


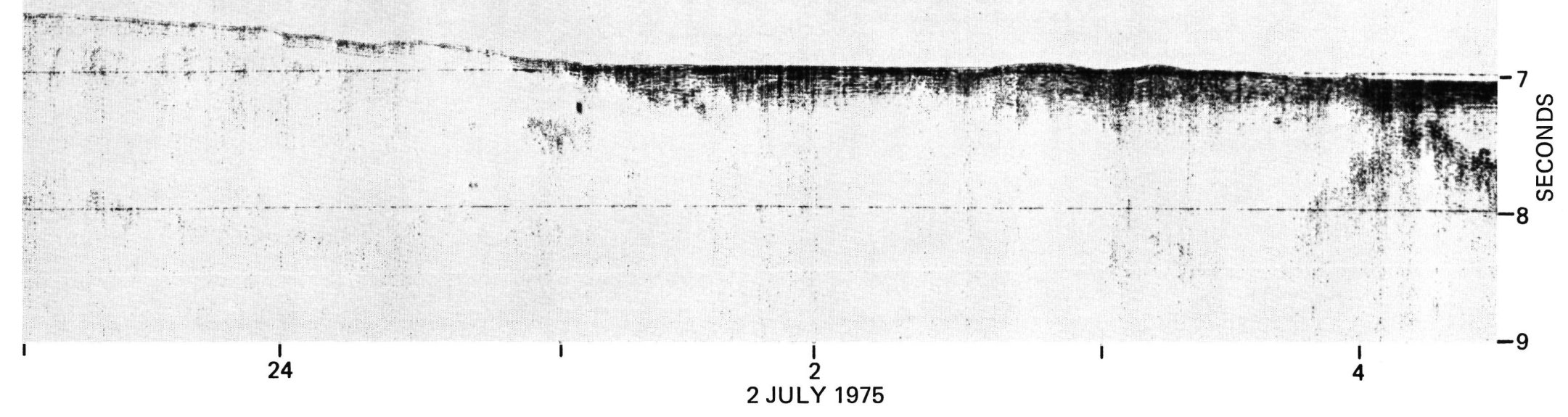

38

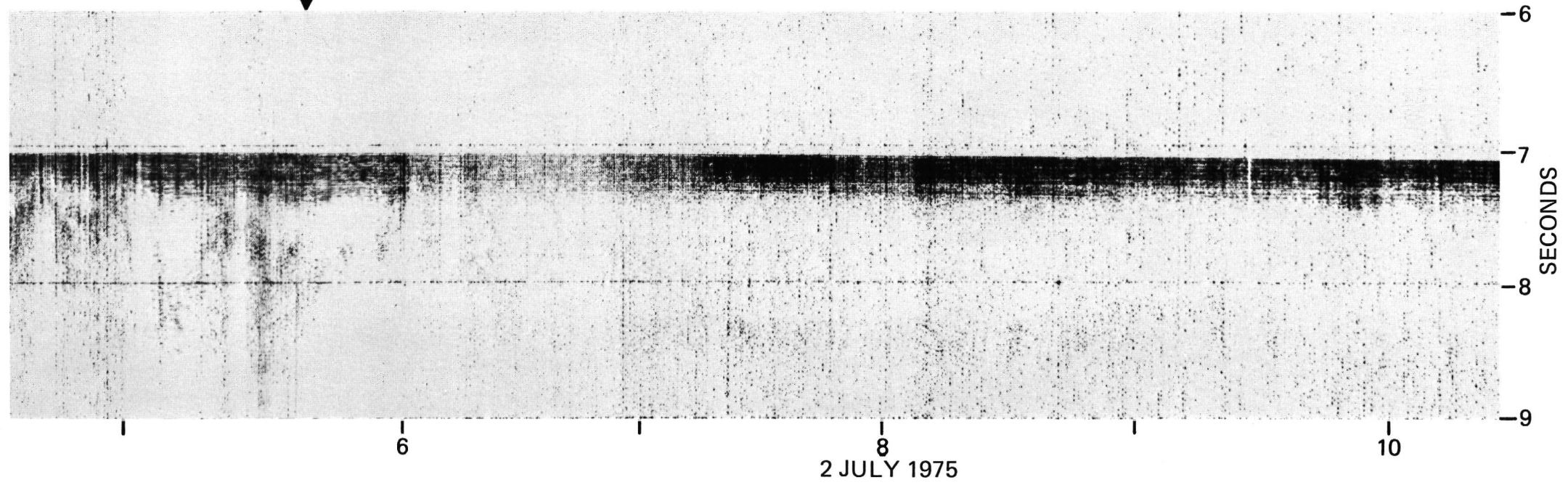

Figure 2. (Continued). 


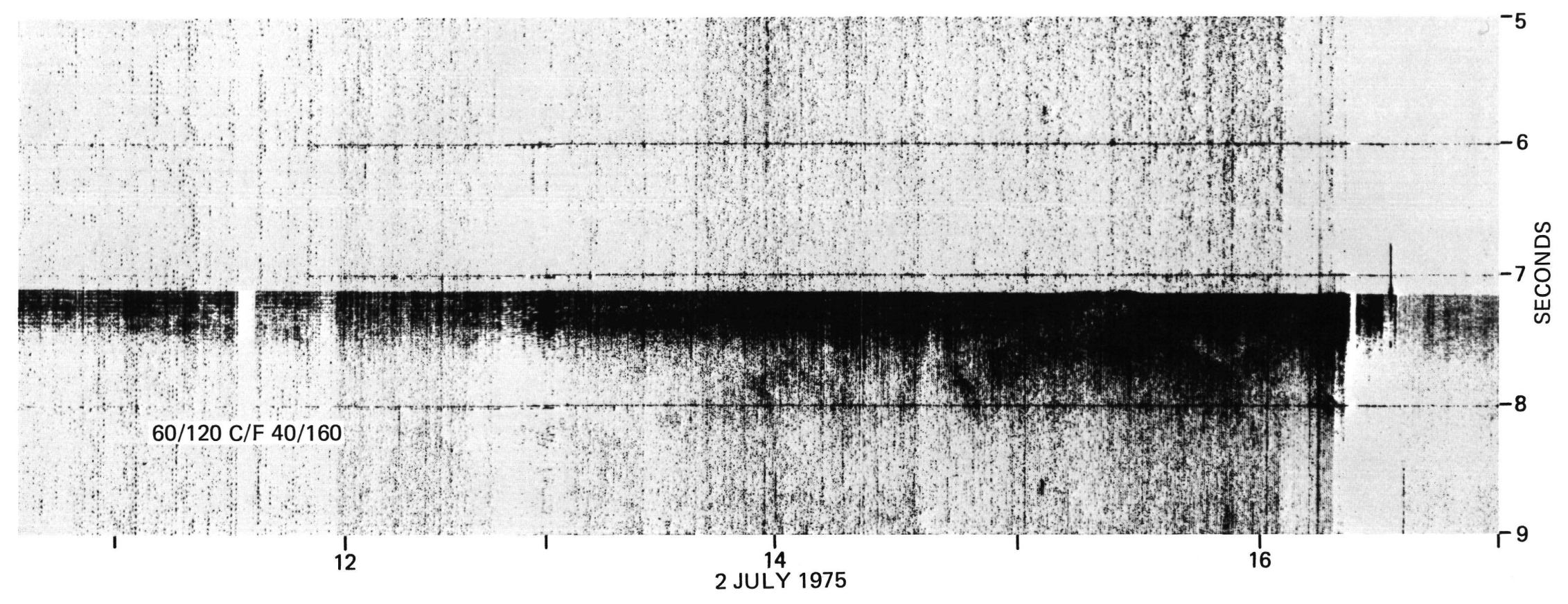

39

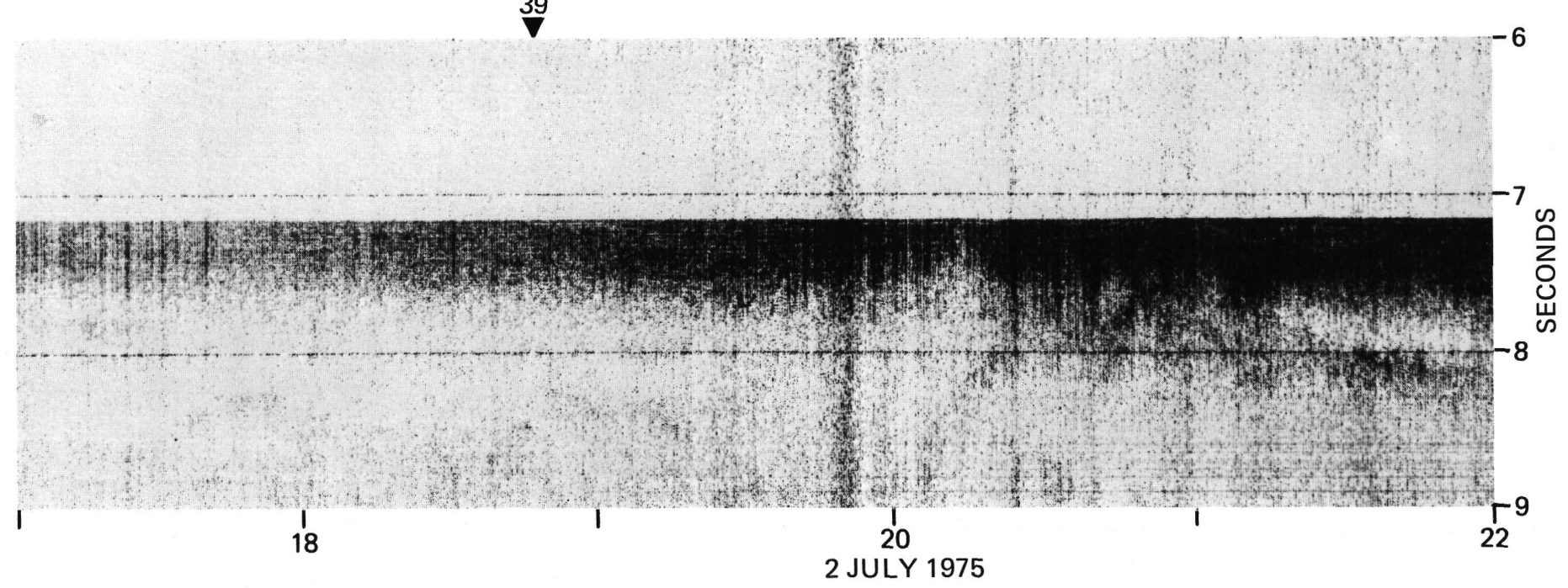

Figure 2. (Continued). 

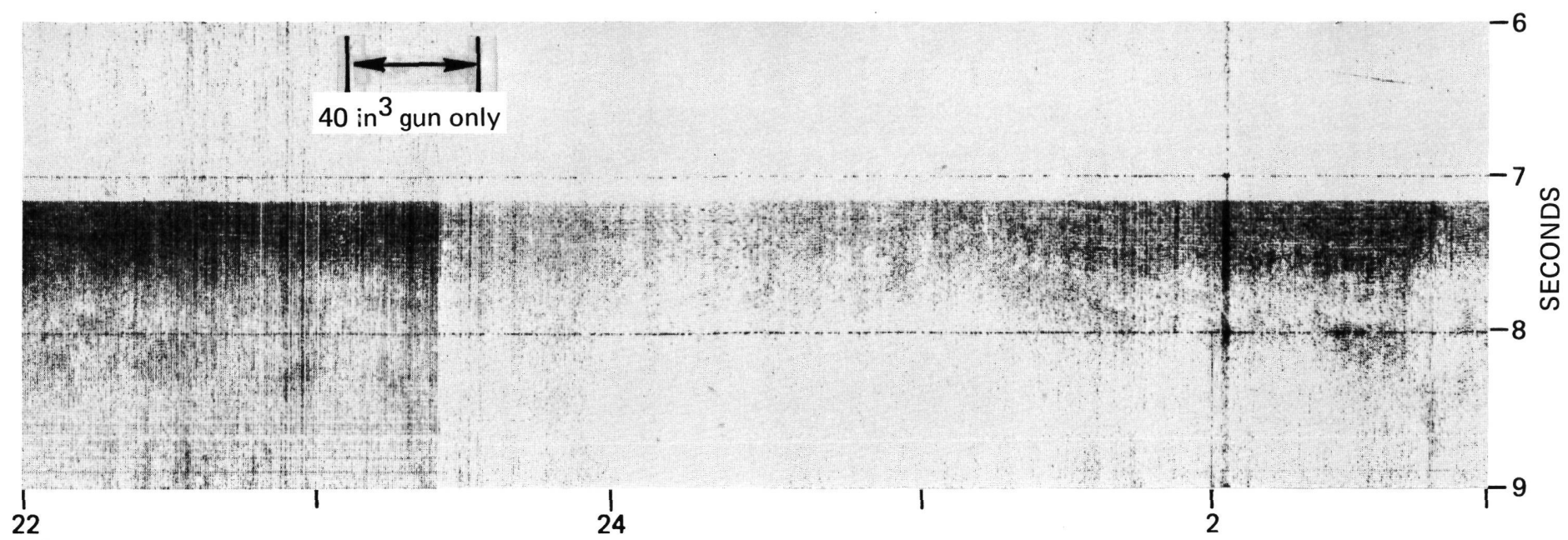

2 JULY 1975

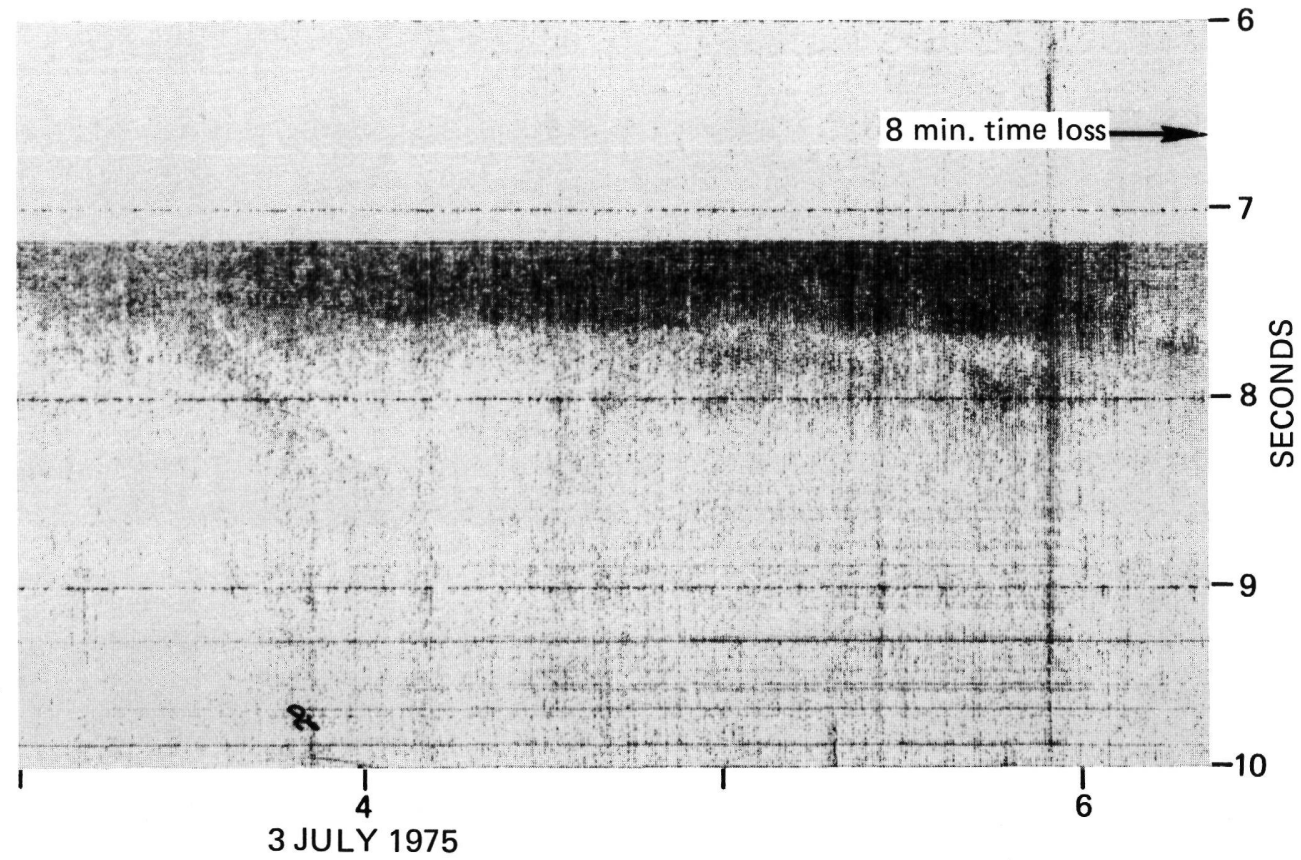

Figure 2. (Continued). 

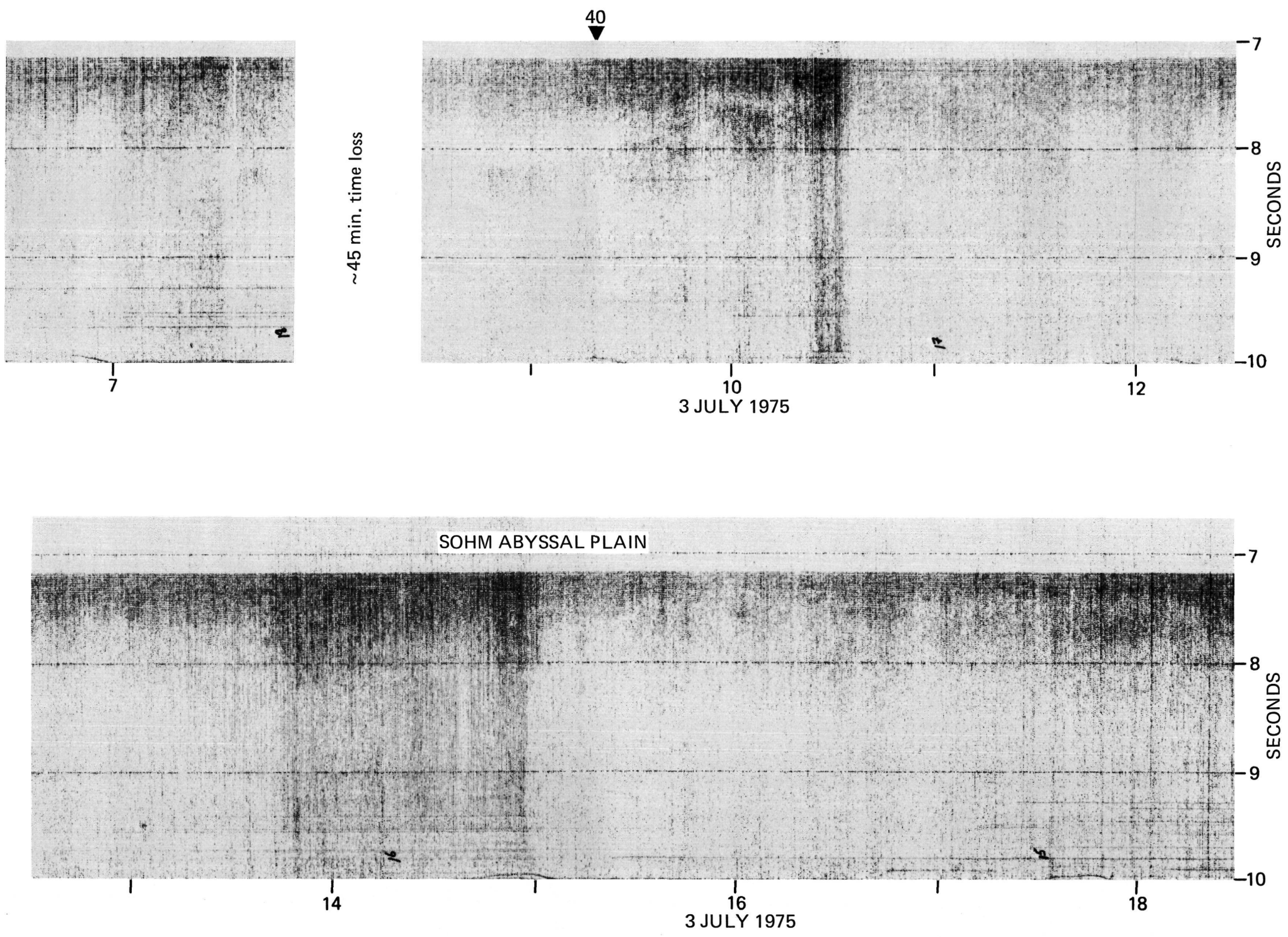

Figure 2. (Continued). 


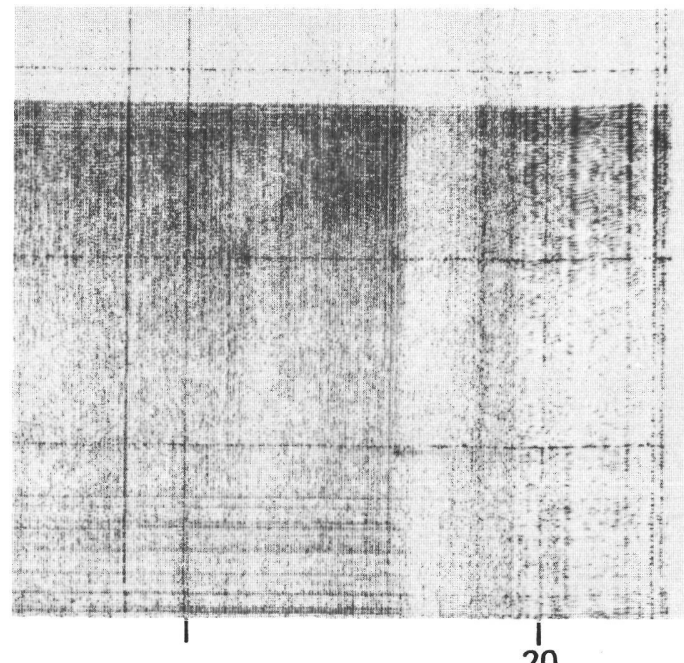

20

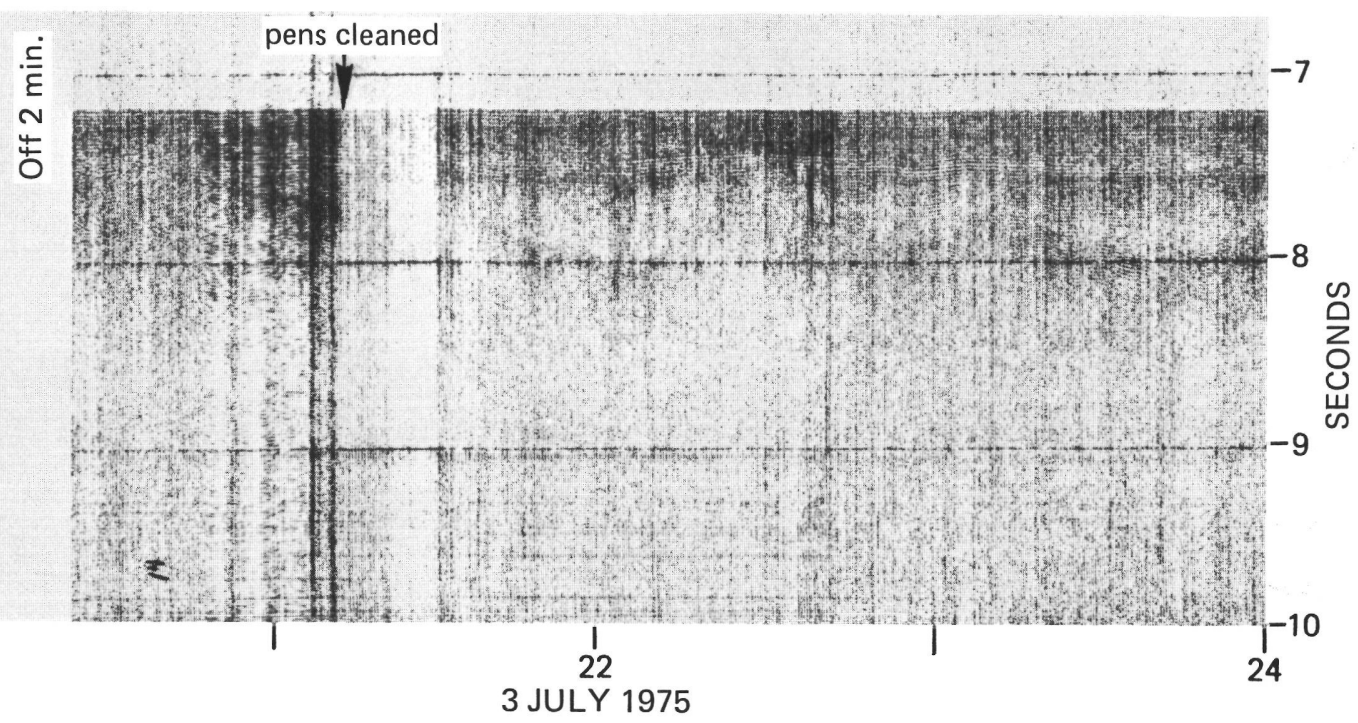

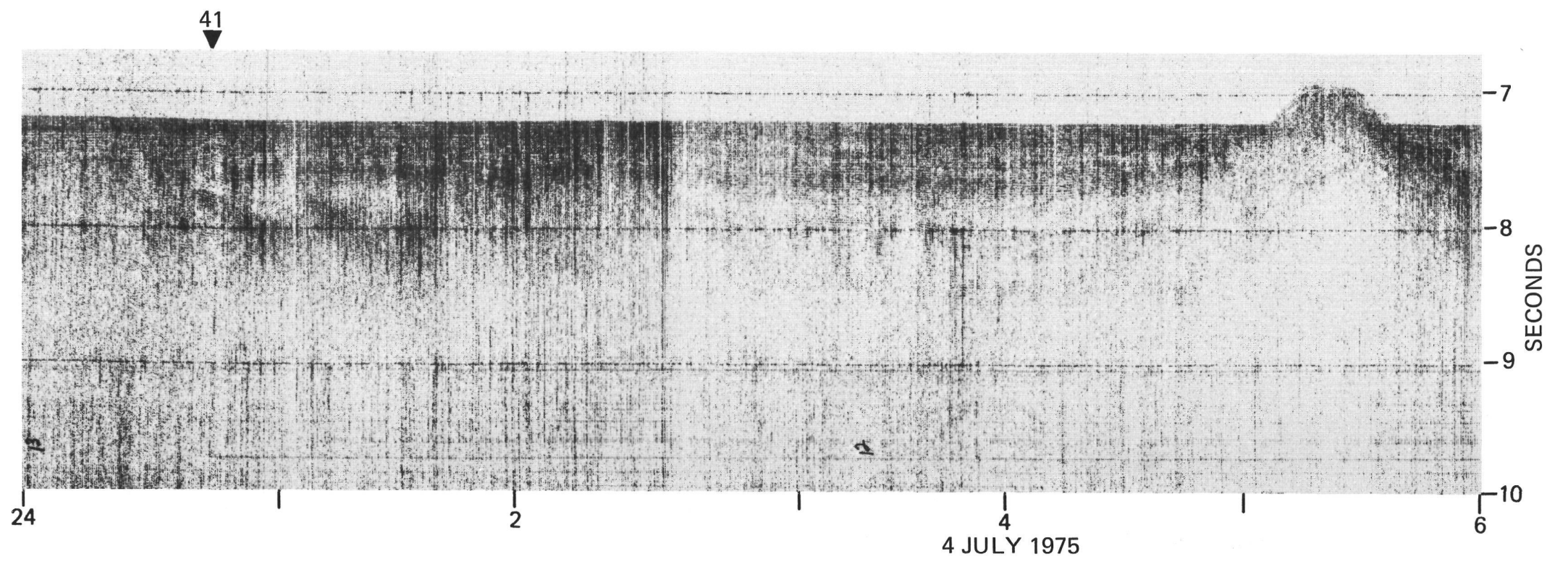

Figure 2. (Continued). 

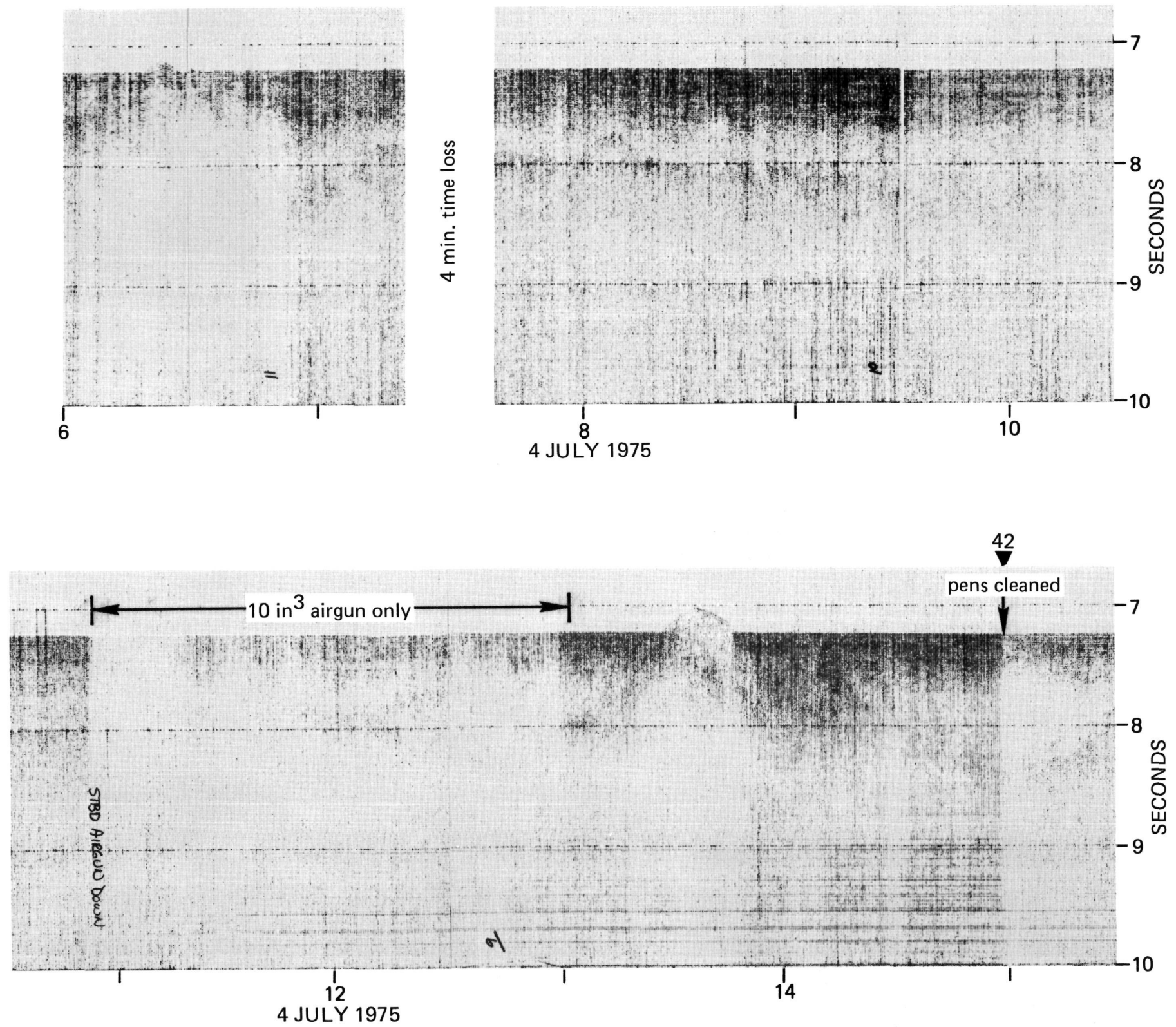

Figure 2. (Continued). 
40 in $^{3}$ gun only

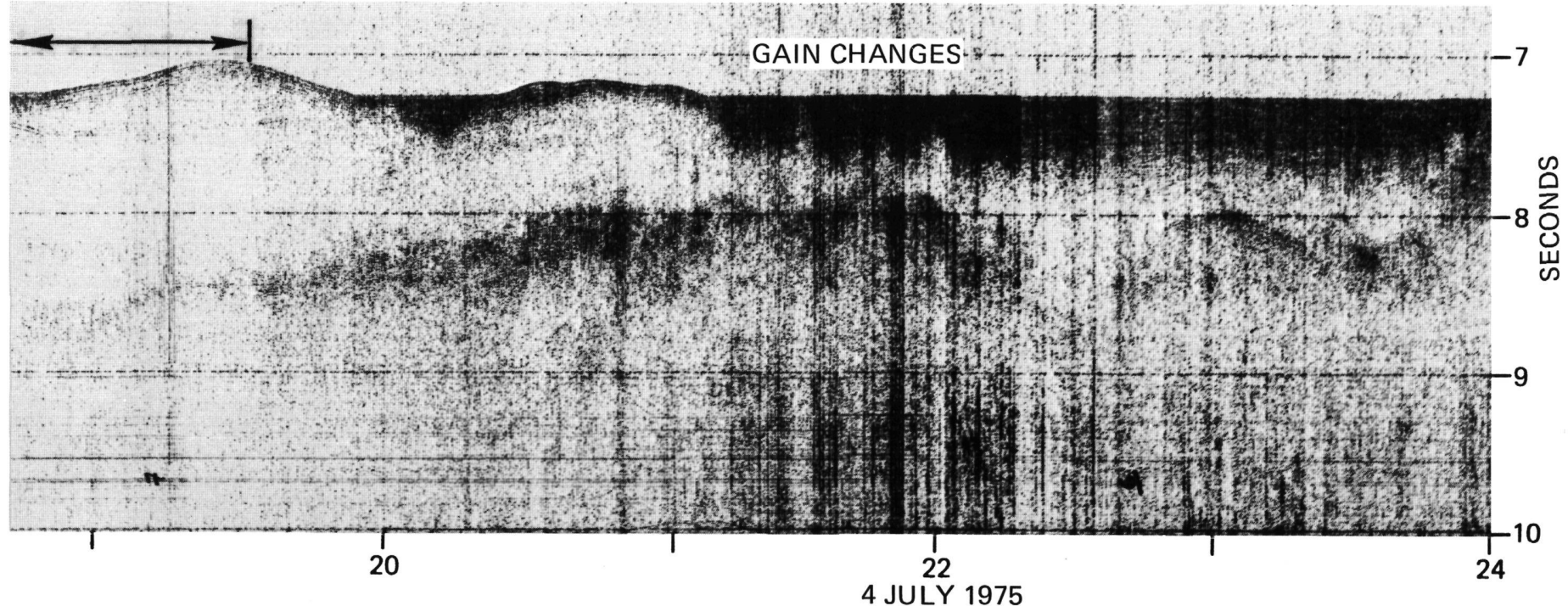

Figure 2. (Continued). 


\section{3}
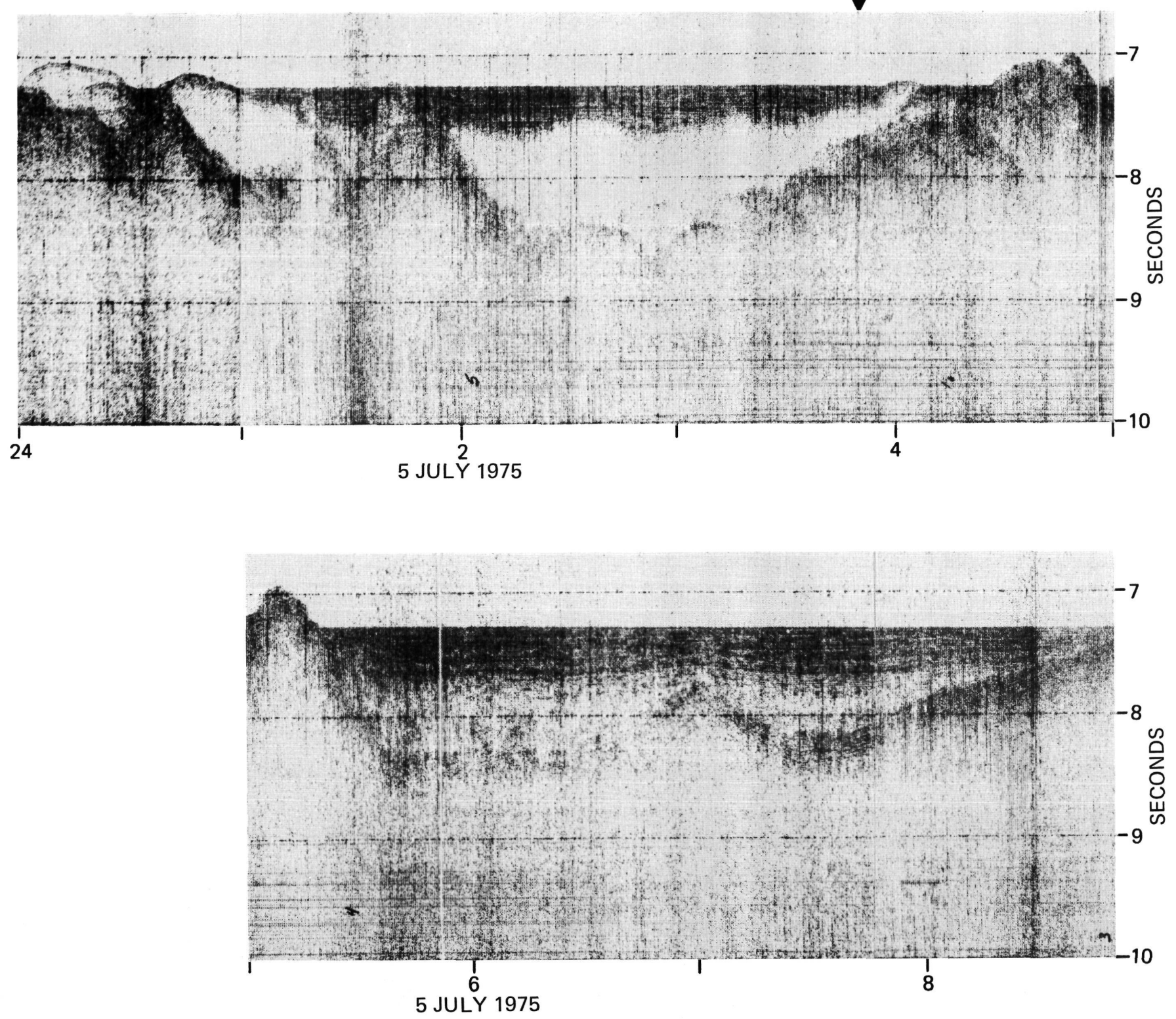

Figure 2. (Continued). 


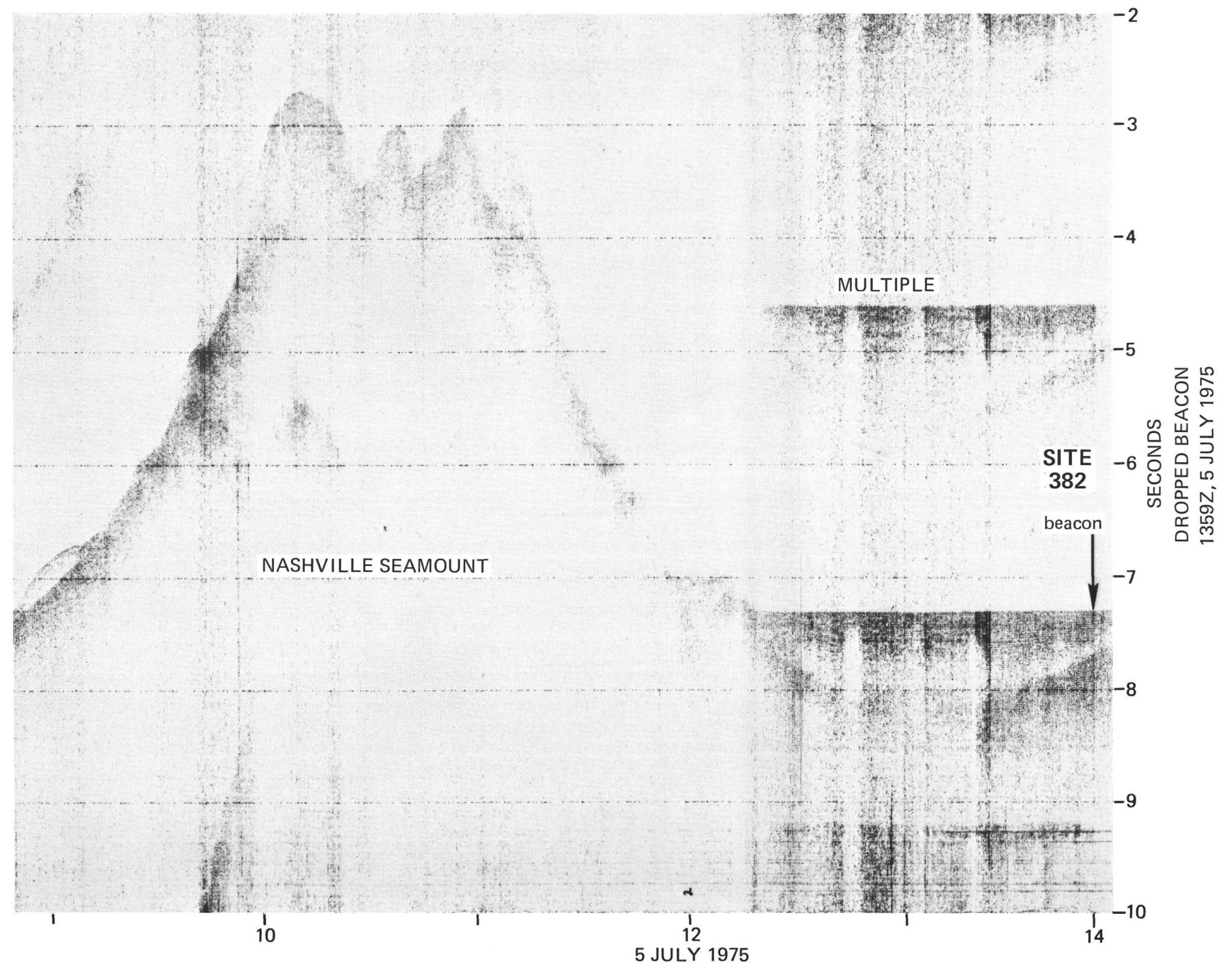

Figure 2. (Continued). 


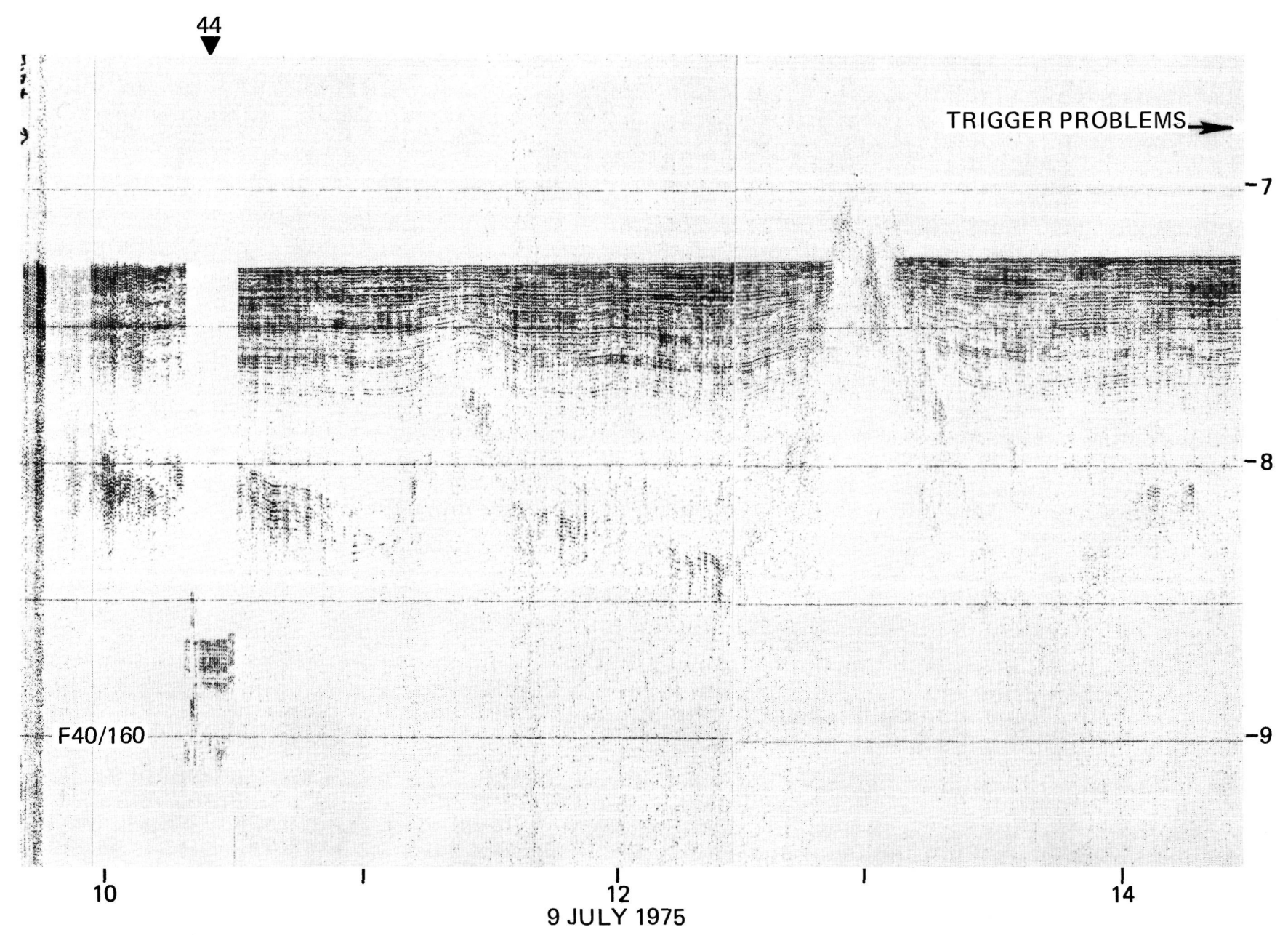

Figure 2. (Continued). 

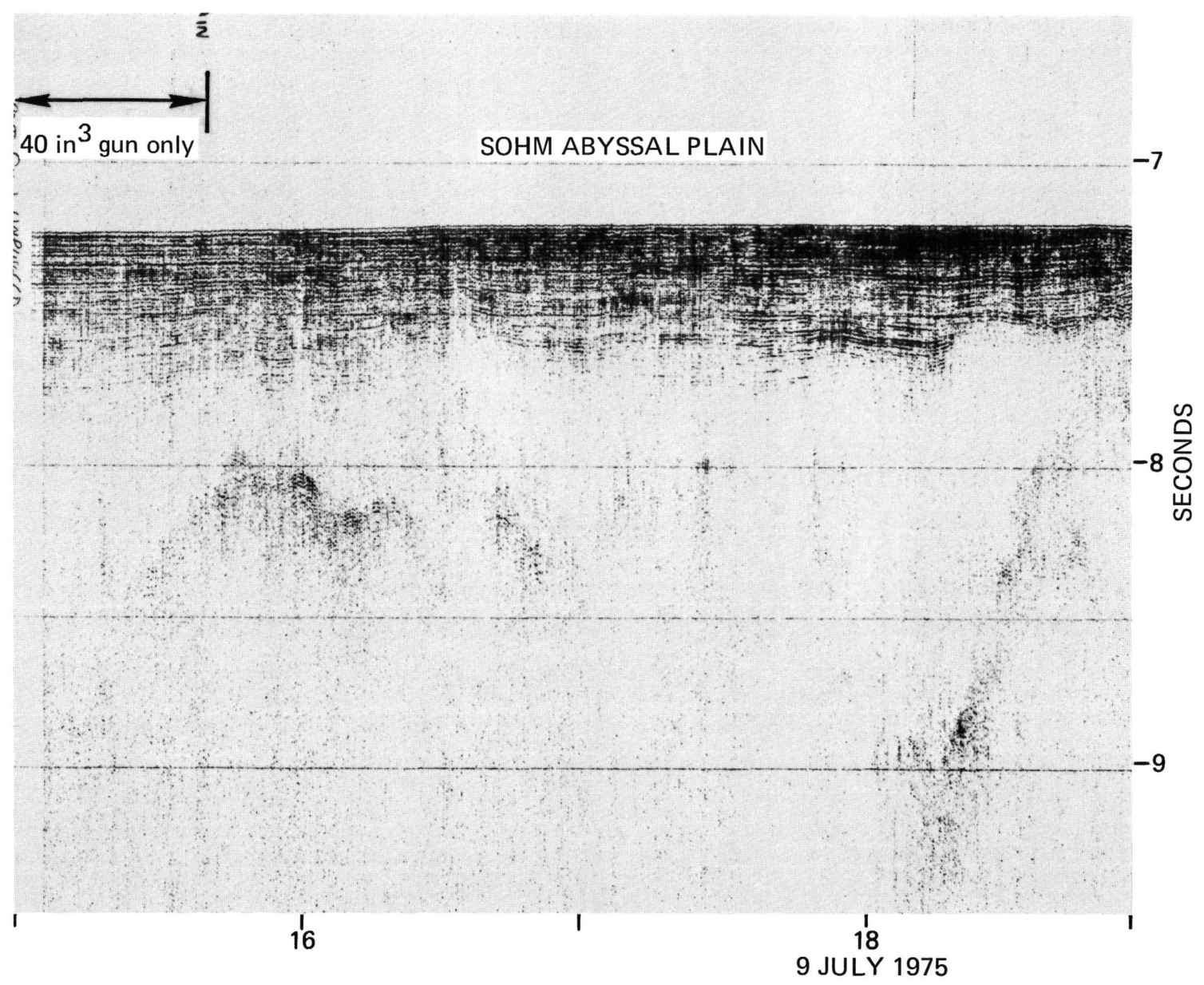

Figure 2. (Continued). 


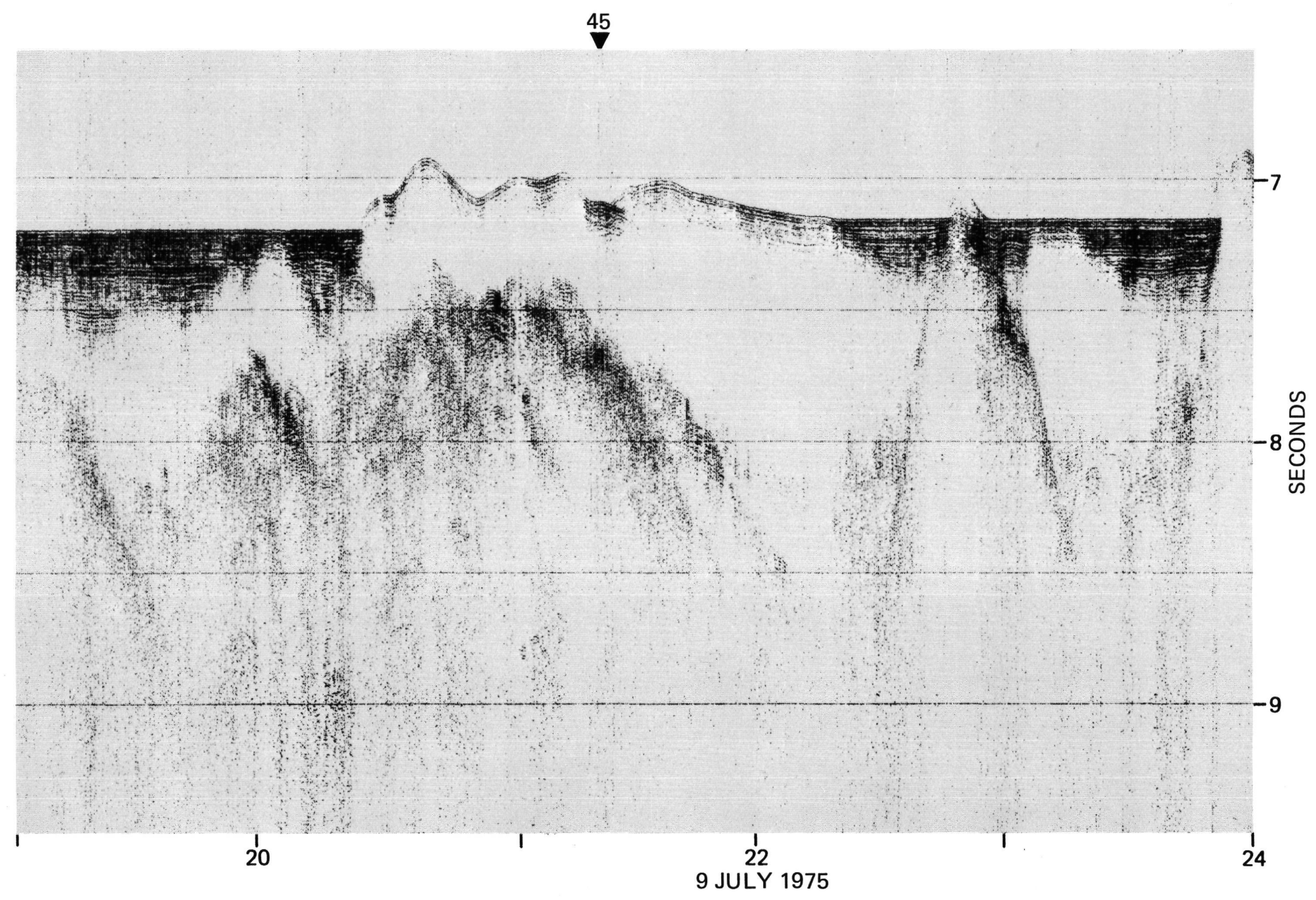

Figure 2. (Continued). 

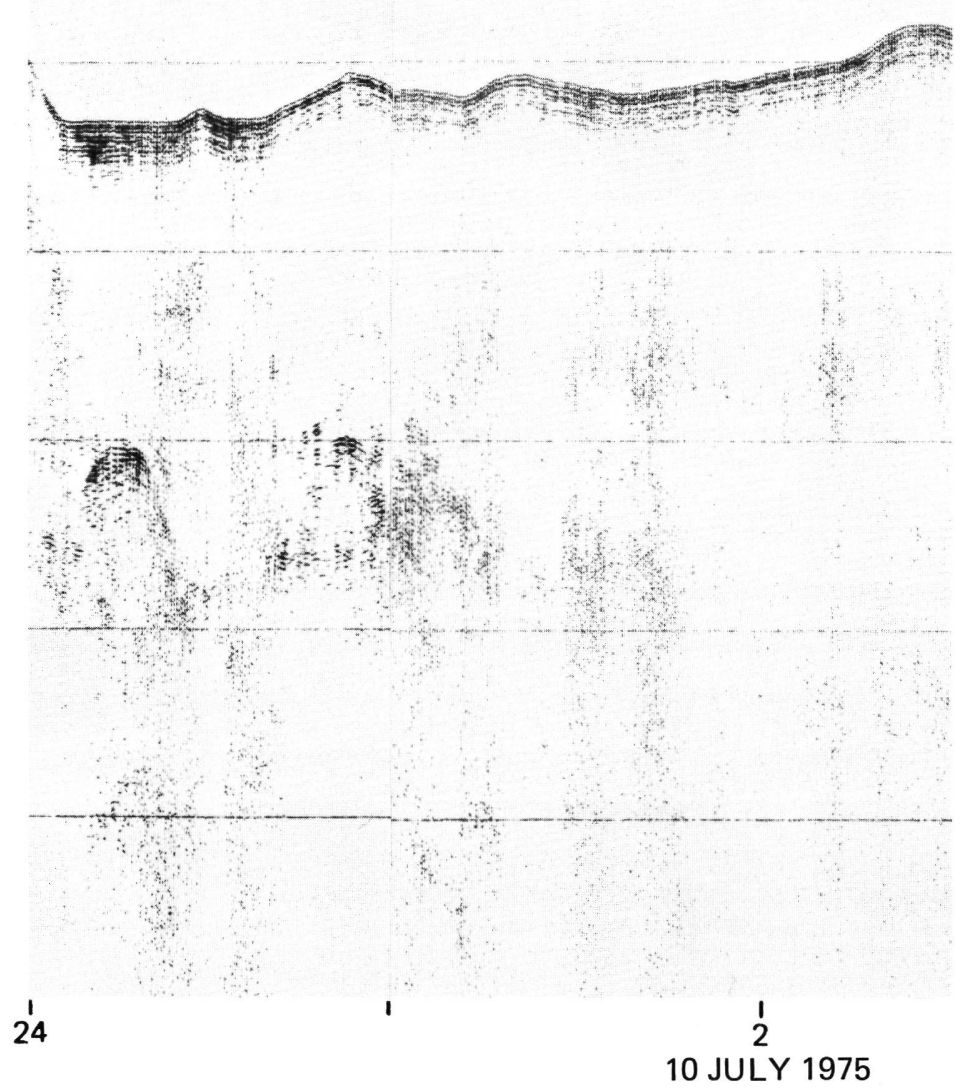

Figure 2. (Continued).

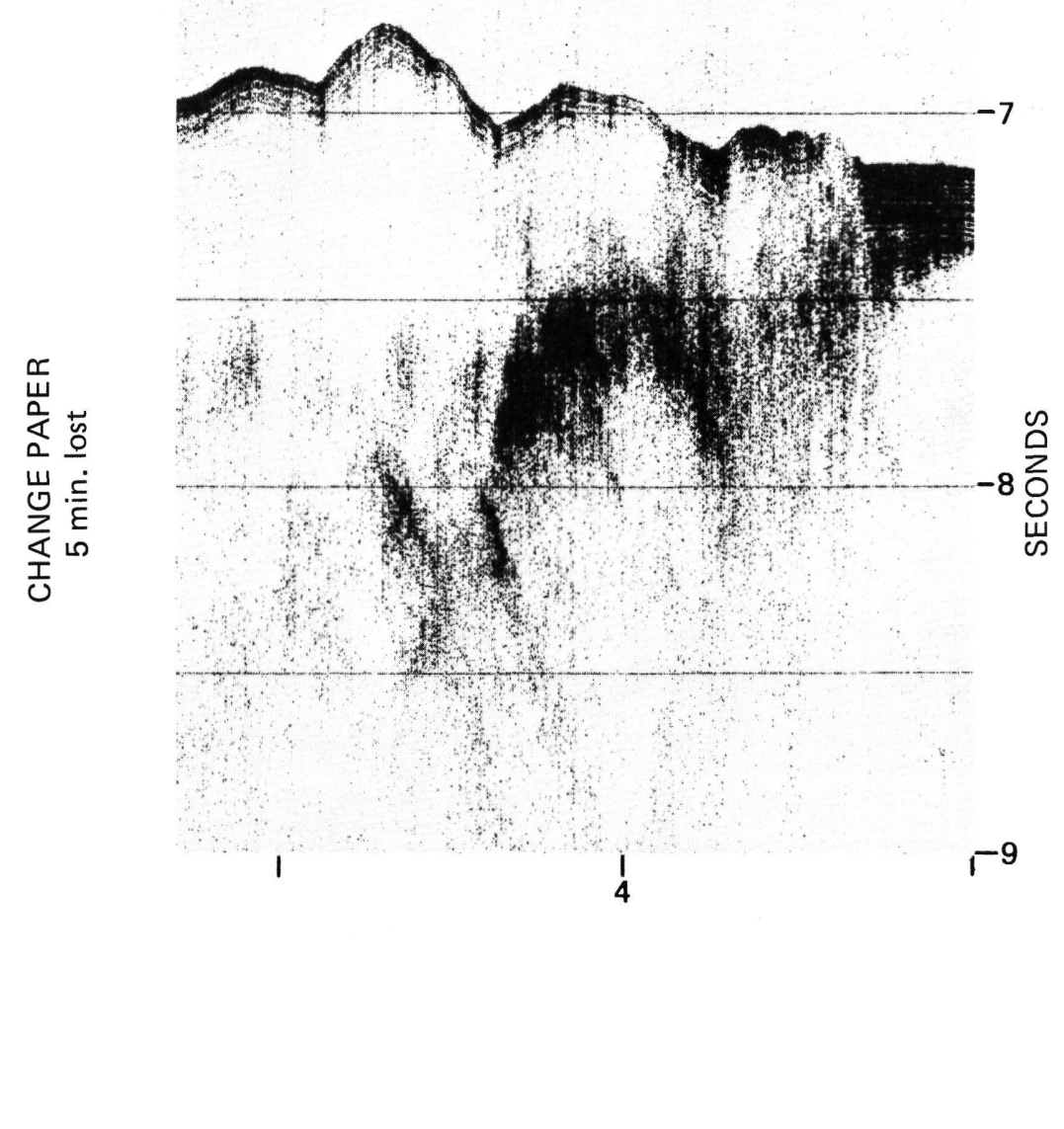




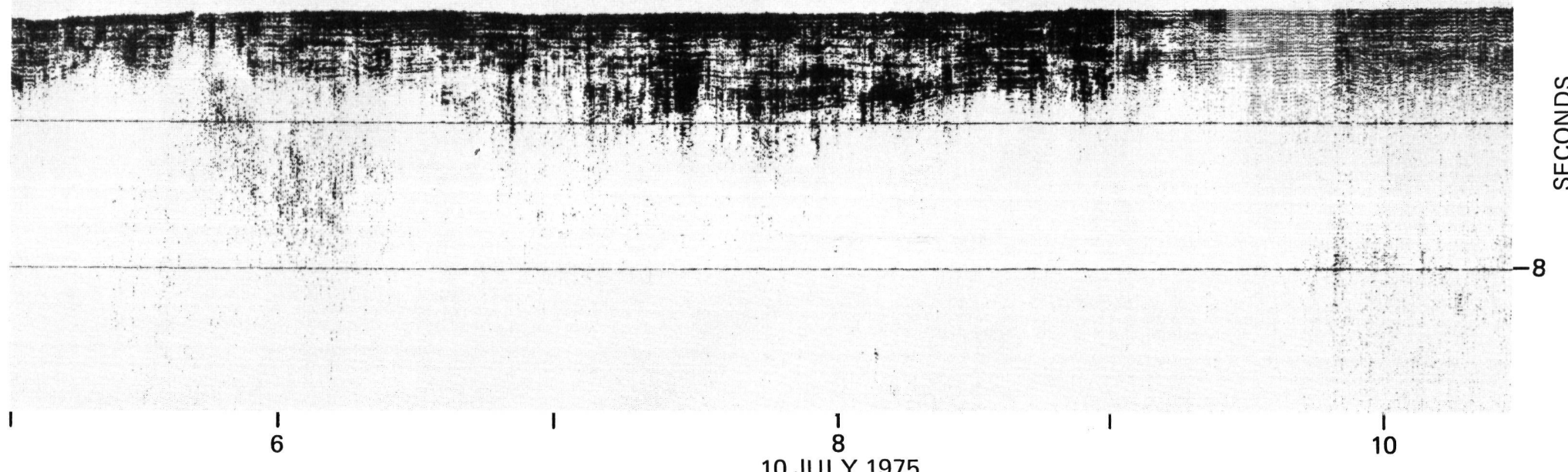

Figure 2. (Continued). 


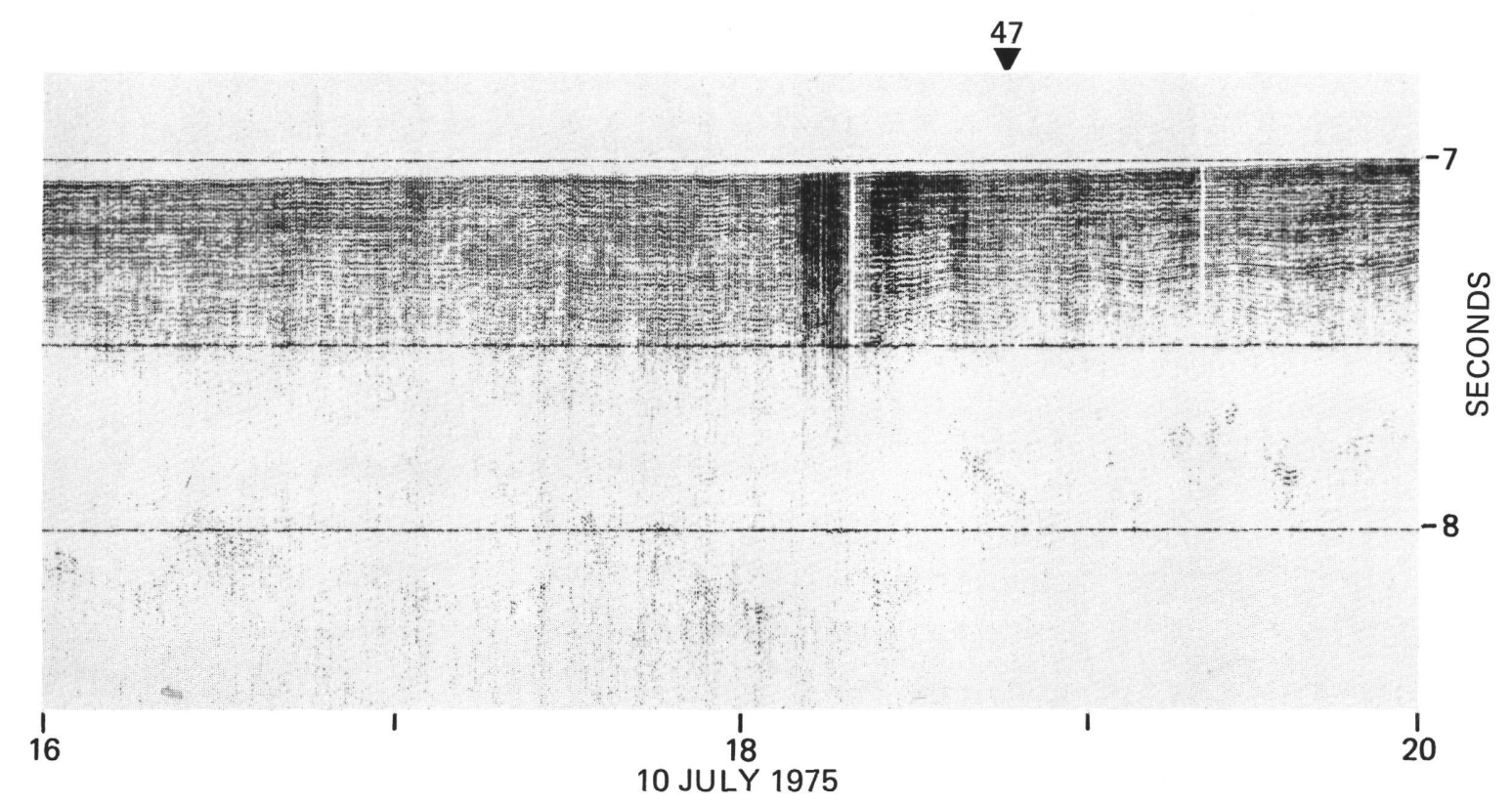

SITE 383

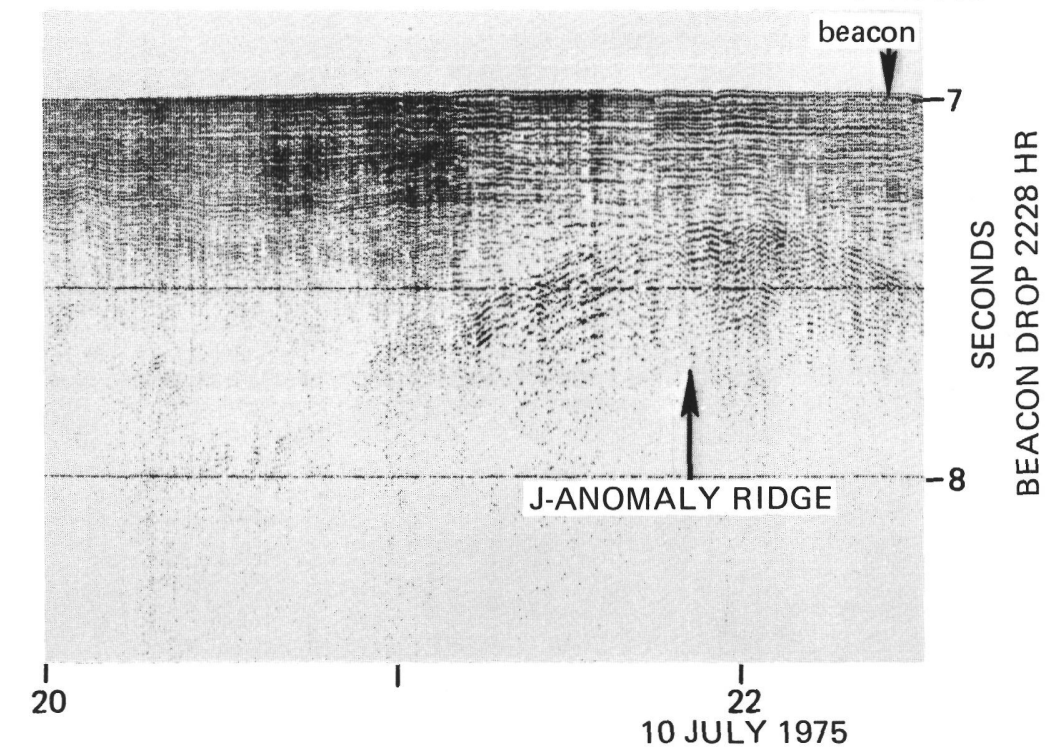

Figure 2. (Continued). 


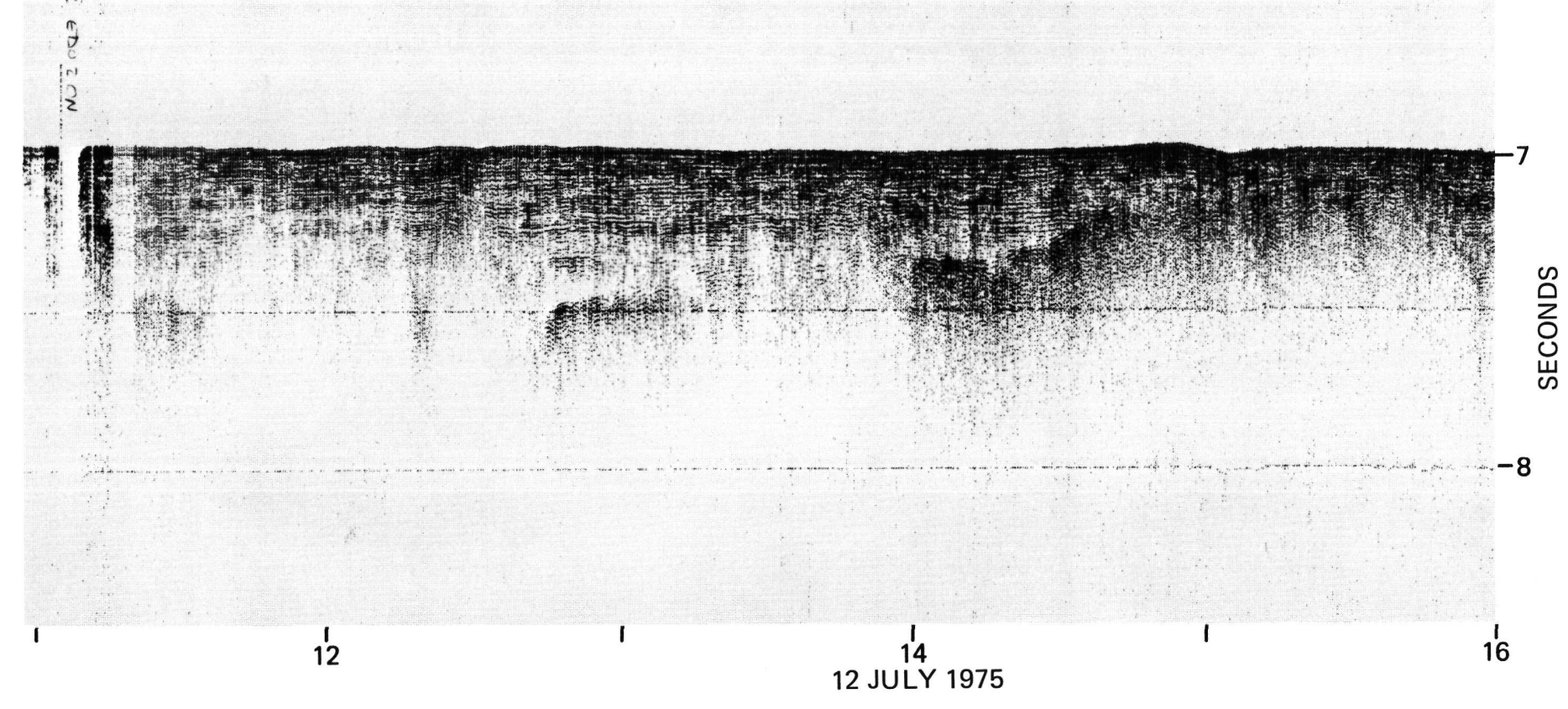

Figure 2. (Continued), 

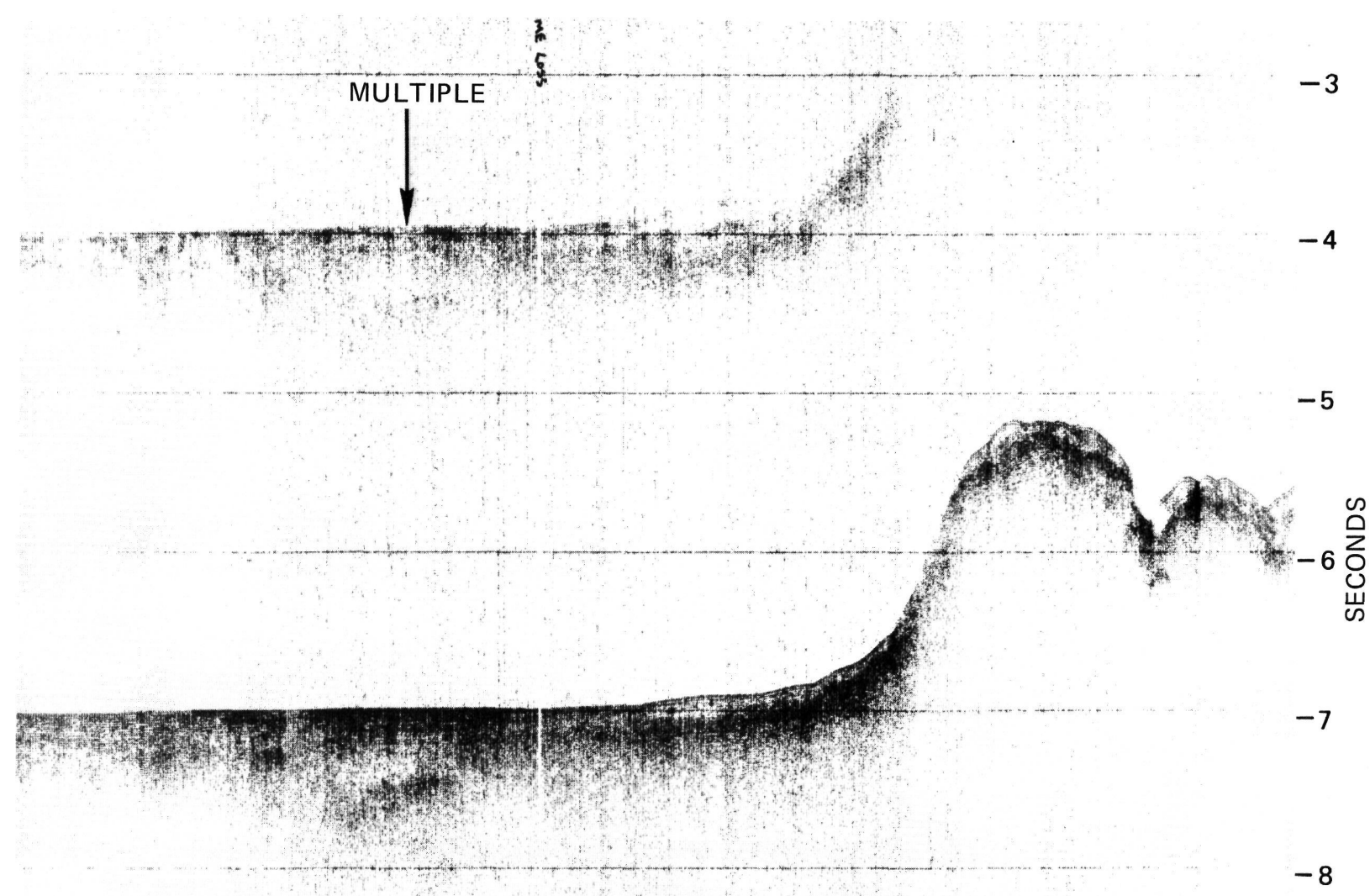

16

1 18 12 JULY 1975

Figure 2. (Continued). 


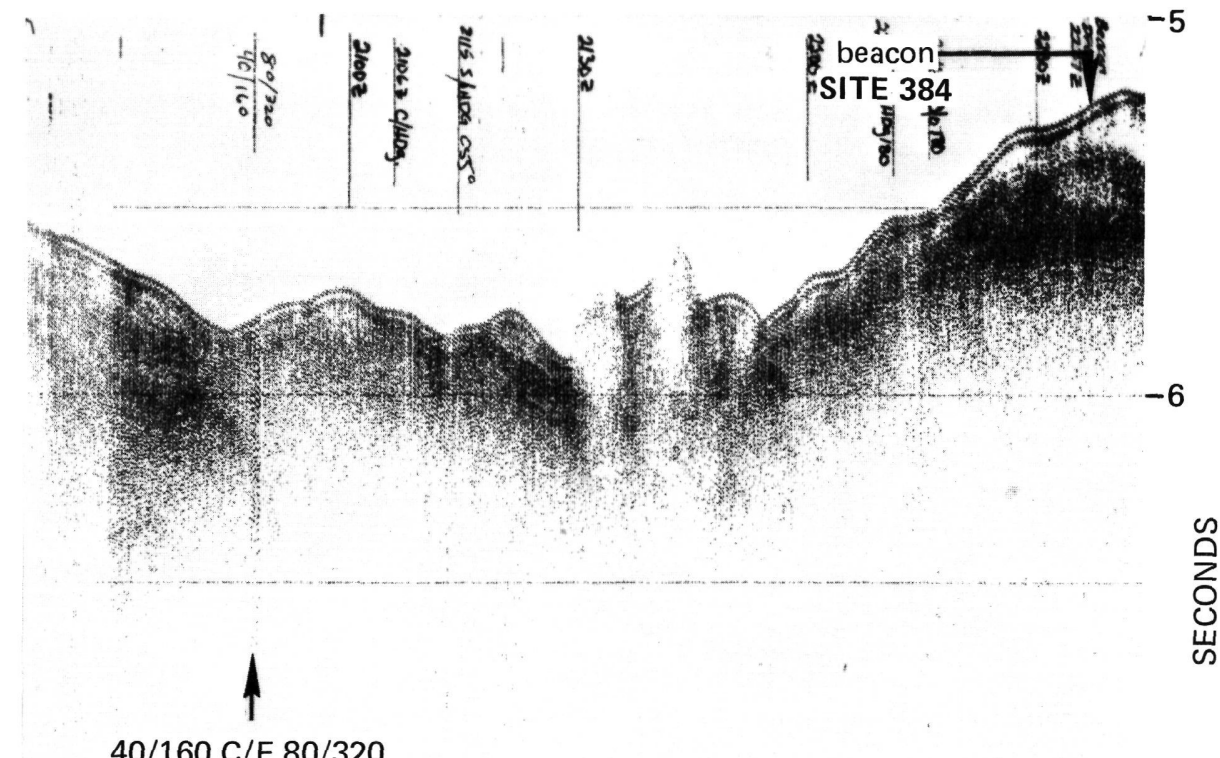

$40 / 160 \mathrm{C} / \mathrm{F} 80 / 320$

号

12 JULY 1975

Figure 2. (Continued). 
SITE 384

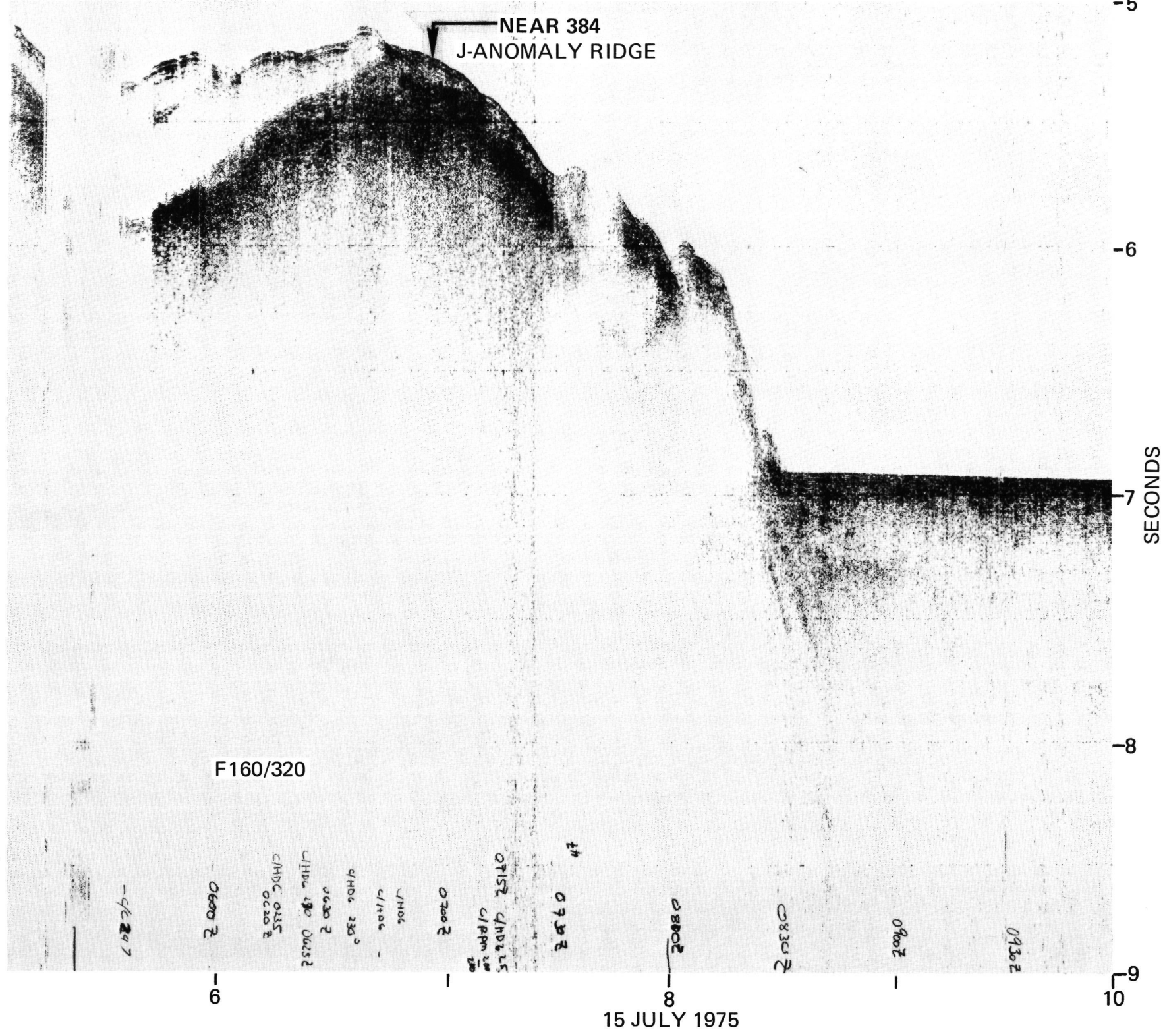

Figure 2. (Continued). 
SOHM ABYSSAL PLAIN
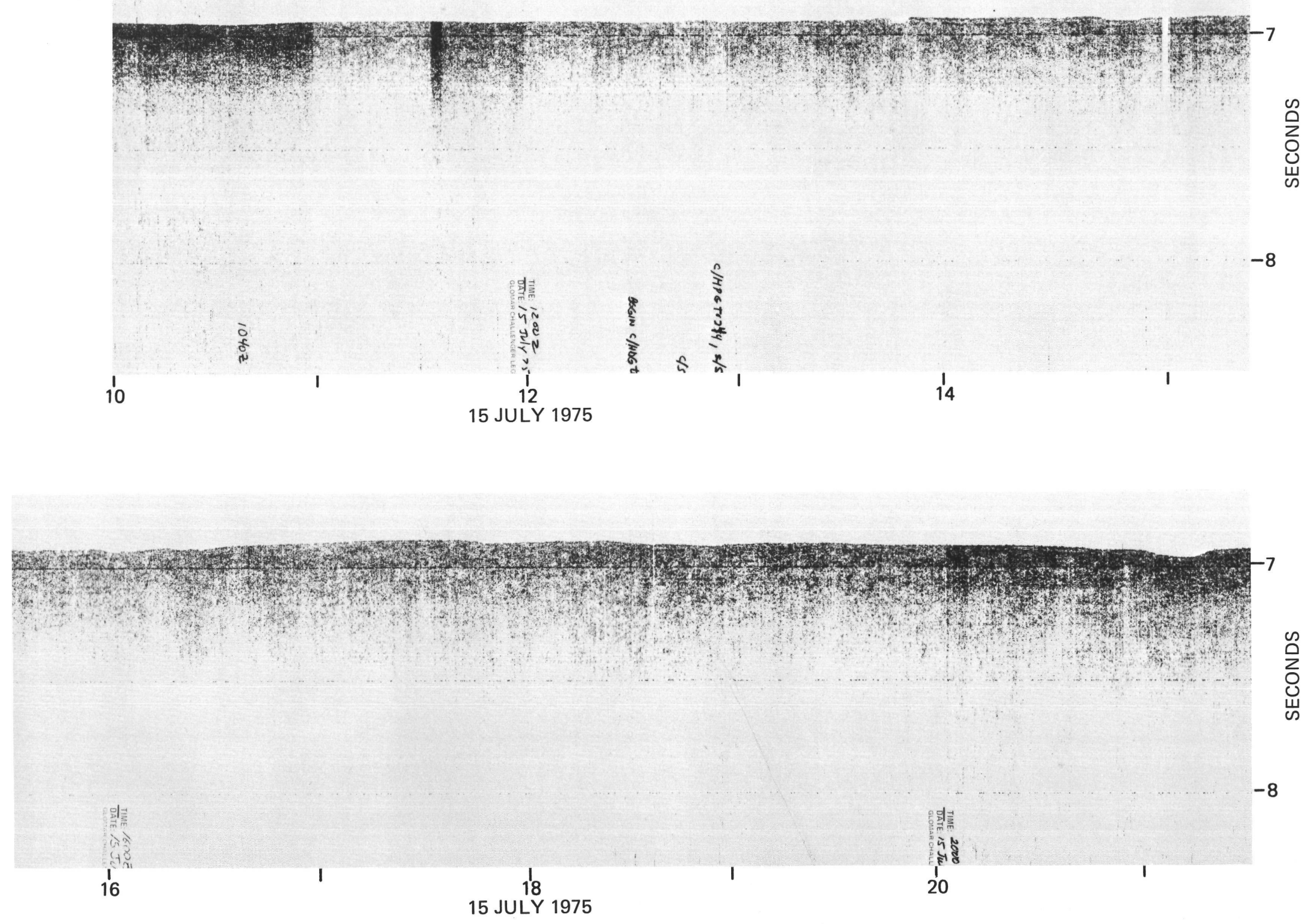

Figure 2. (Continued). 


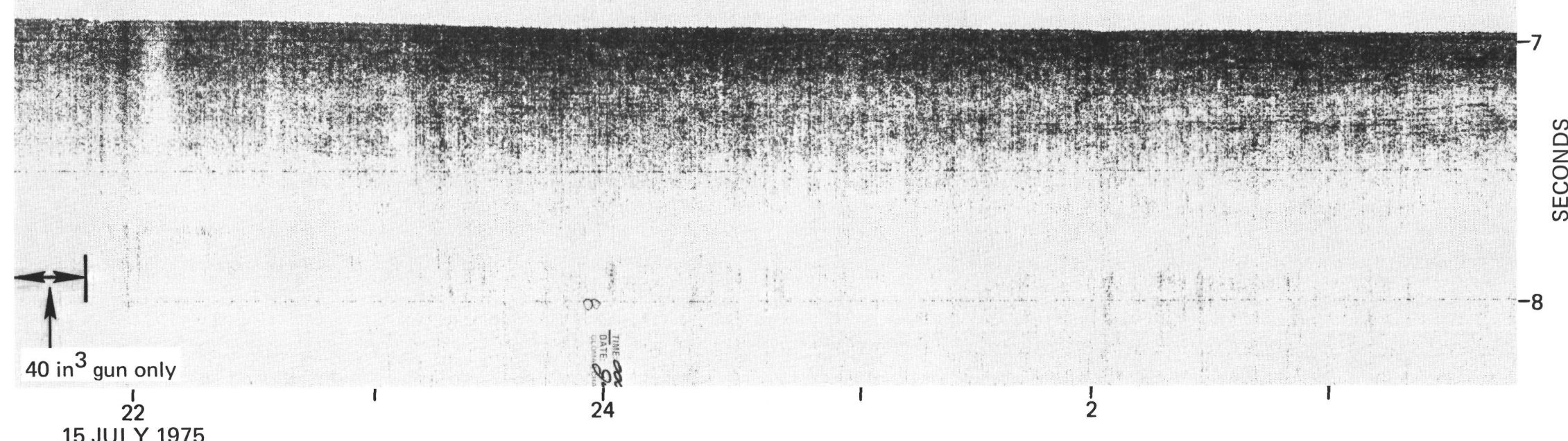

SOHM ABYSSAL PLAIN

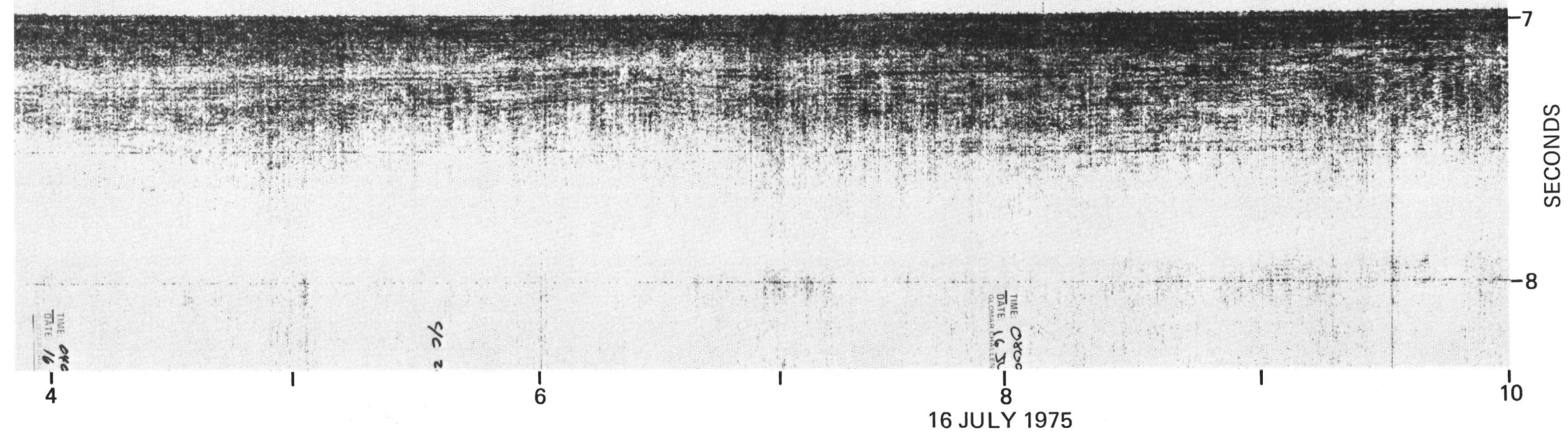

Figure 2. (Continued). 

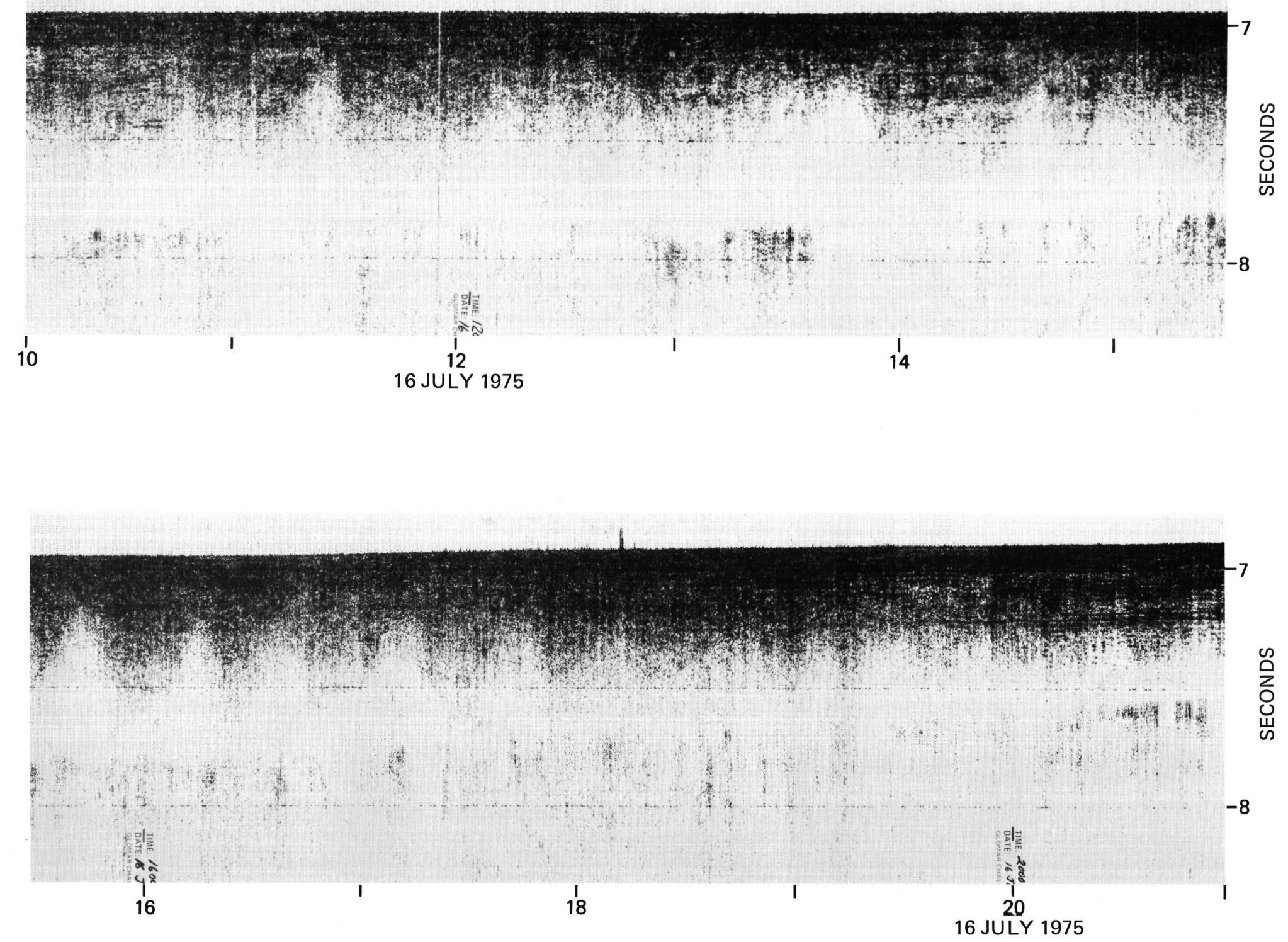

Figure 2. (Continued). 


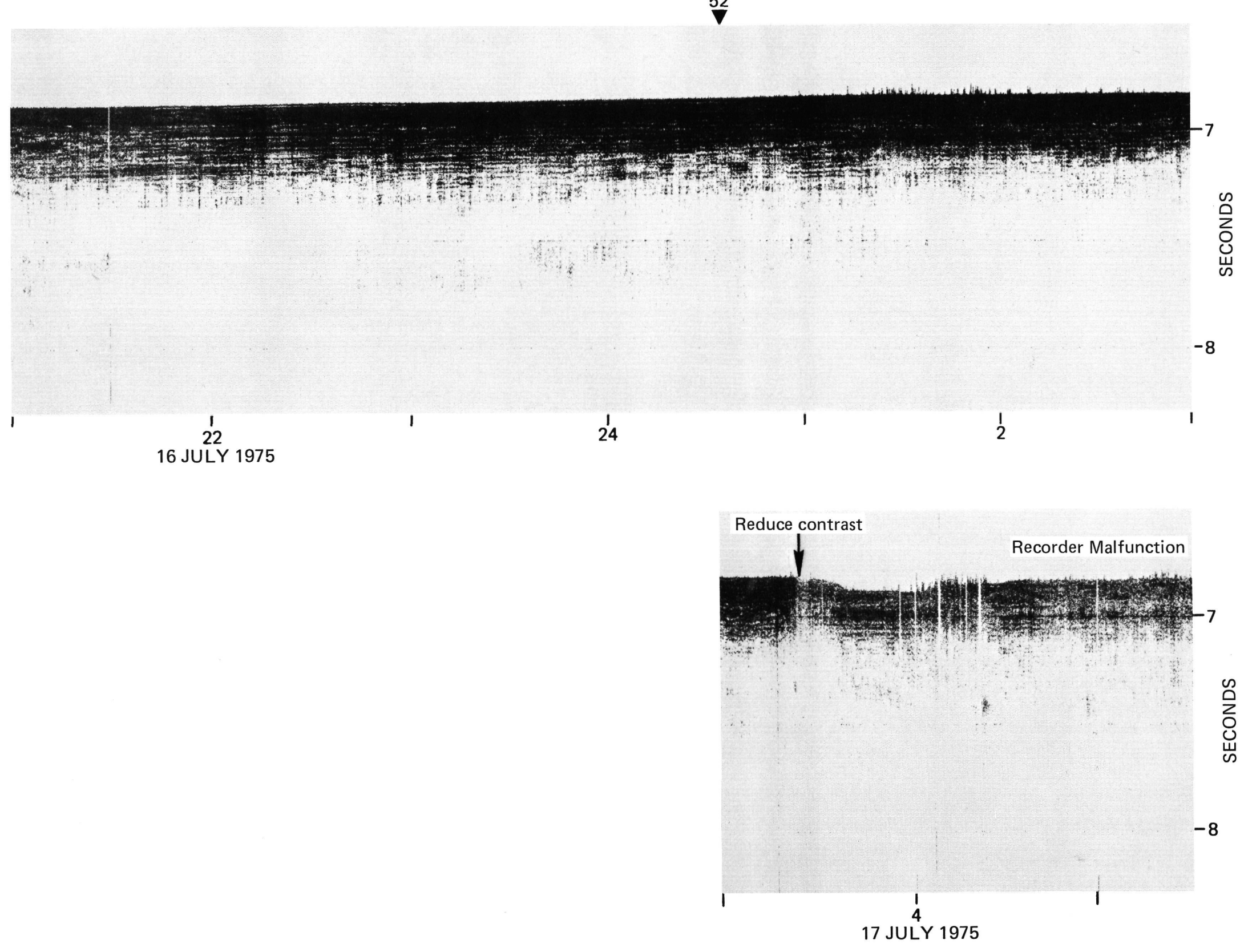

Figure 2. (Continued). 


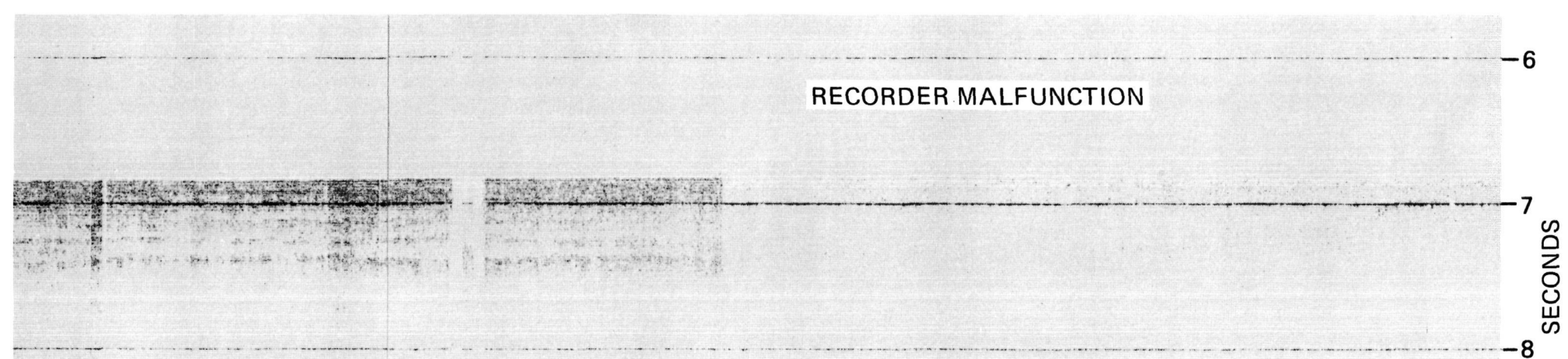

F40/160

6

8

17 JULY 1975

10

$\Gamma^{9}$

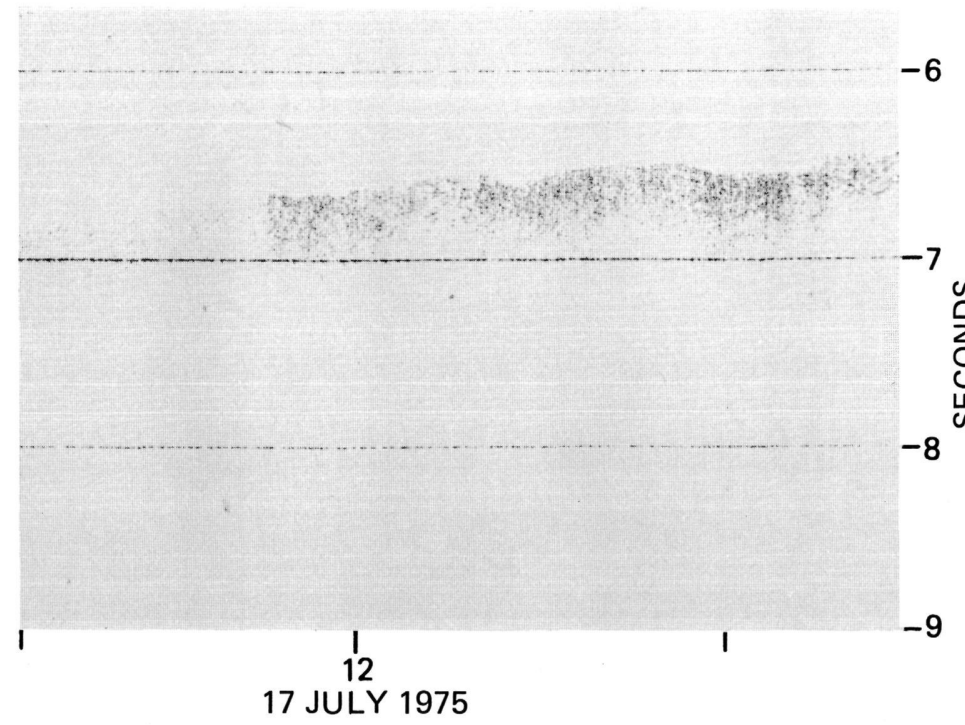

Figure 2. (Continued). 
$\sqrt[53]{ }$

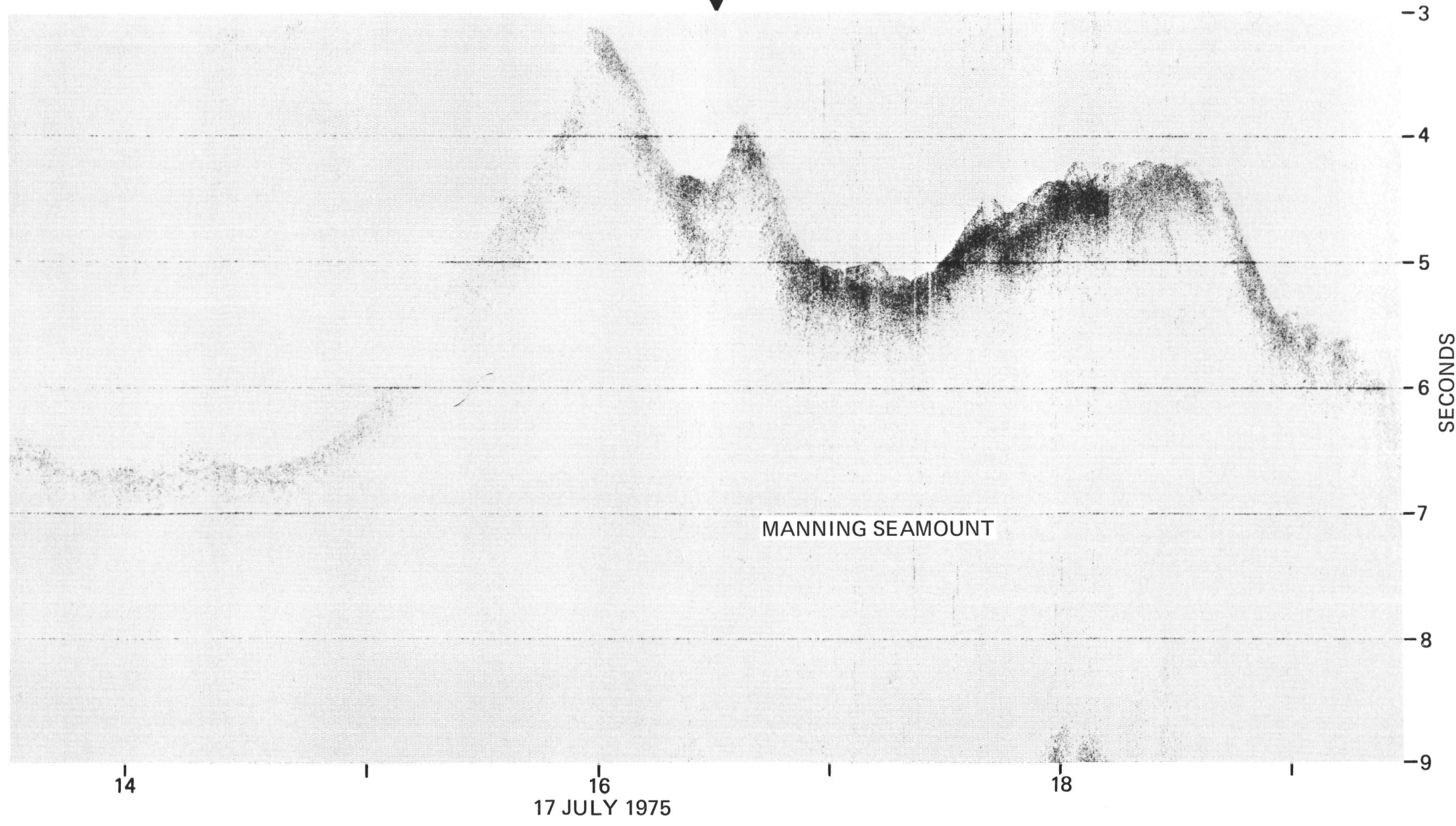

Figure 2. (Continued). 


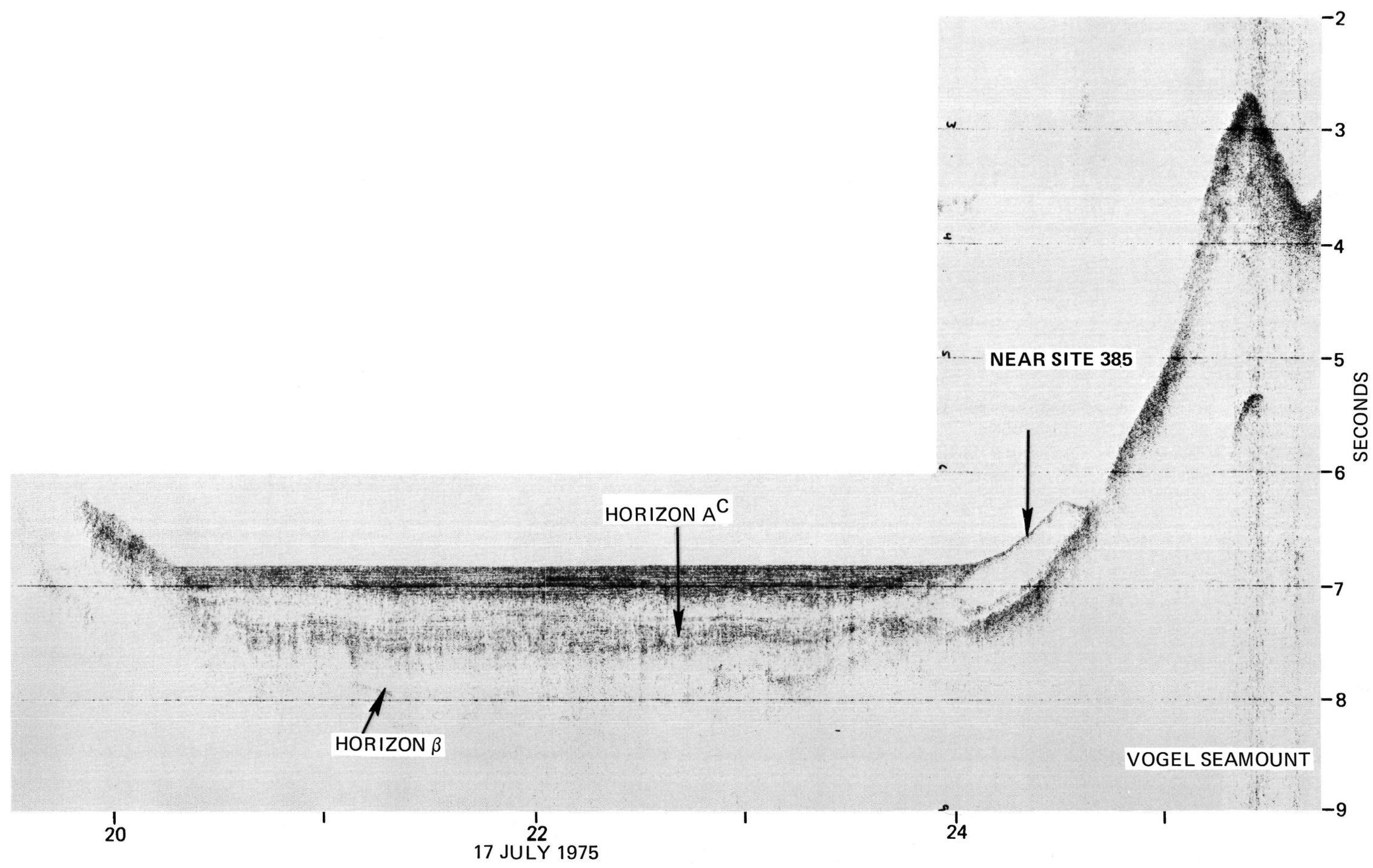

Figure 2. (Continued). 


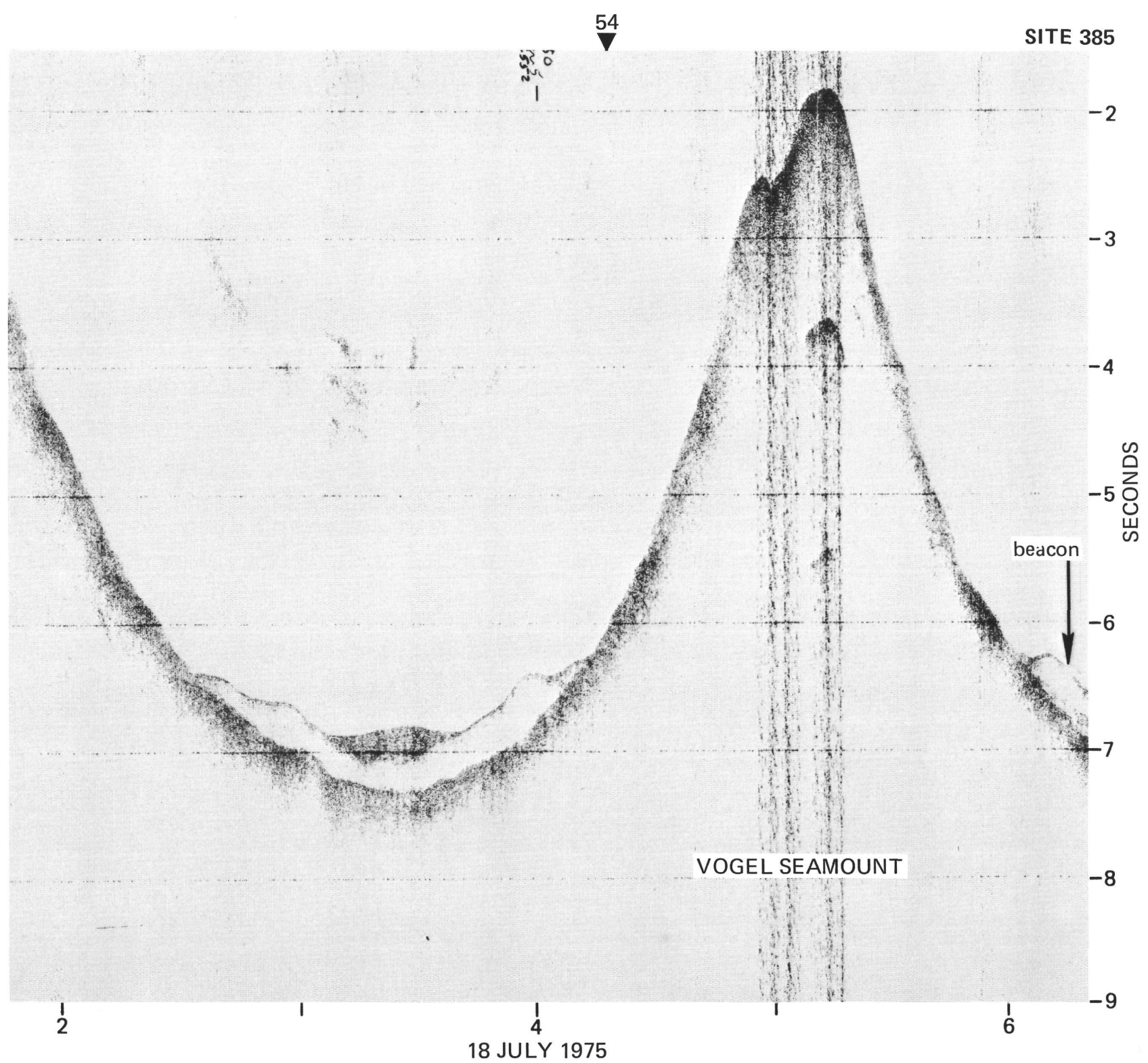

Figure 2. (Continued). 


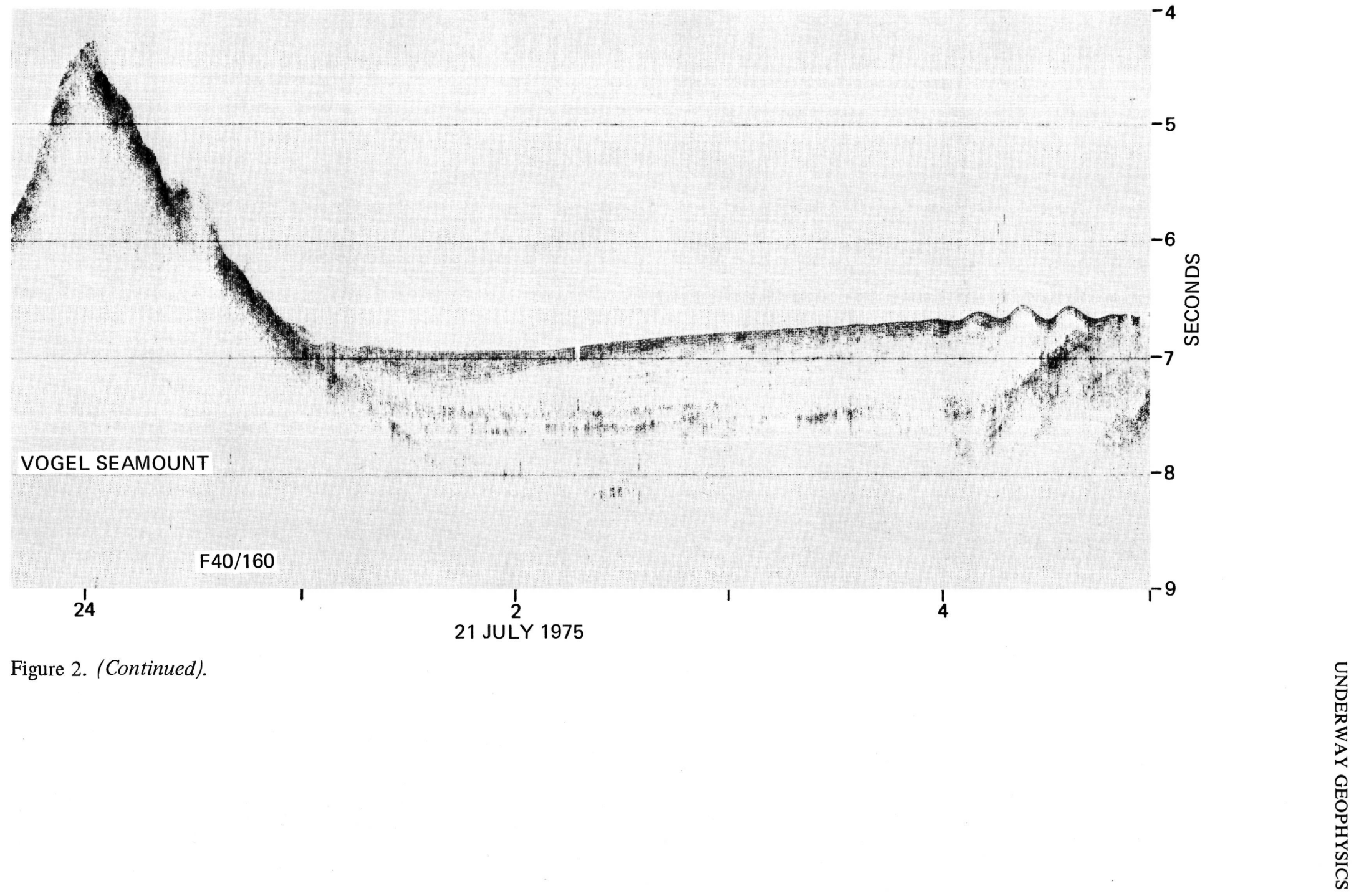



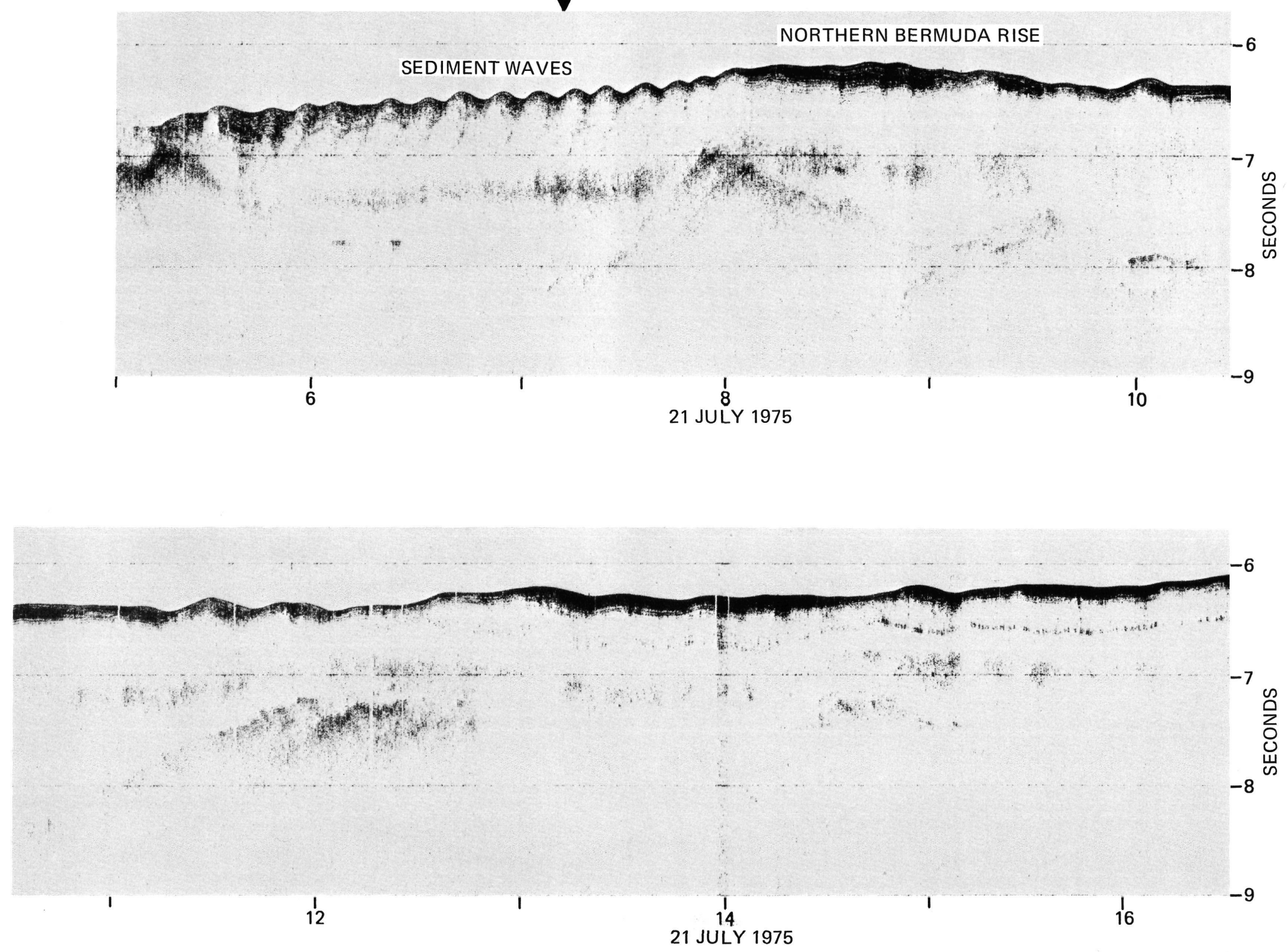

Figure 2. (Continued). 


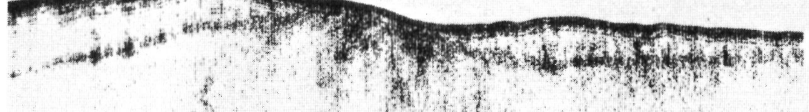

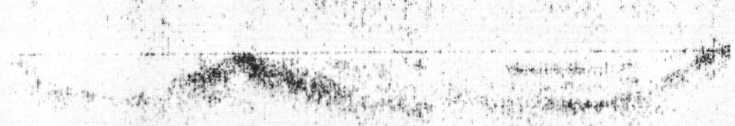

18

21 JULY 1975

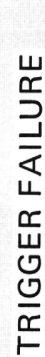

${ }^{\prime} 0$

$-9$

$-5$

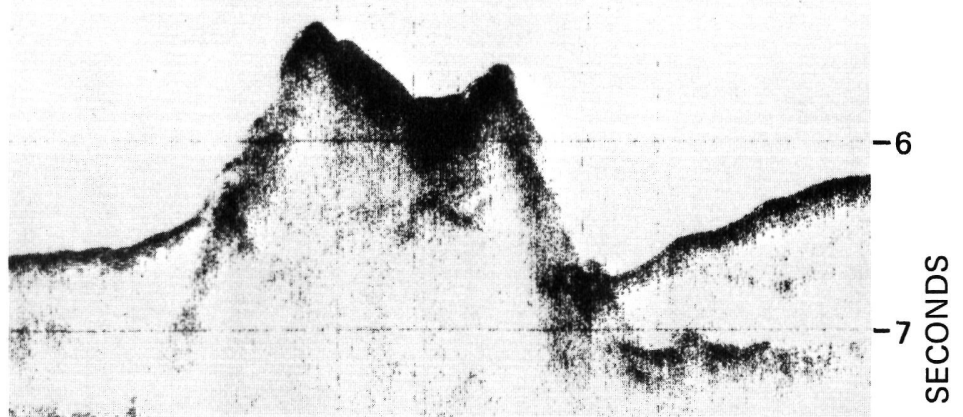

totis

$-8$

${ }^{1-9}$

21 JULY 1975 
<smiles>CC1CCC(CI)CC1C</smiles> 

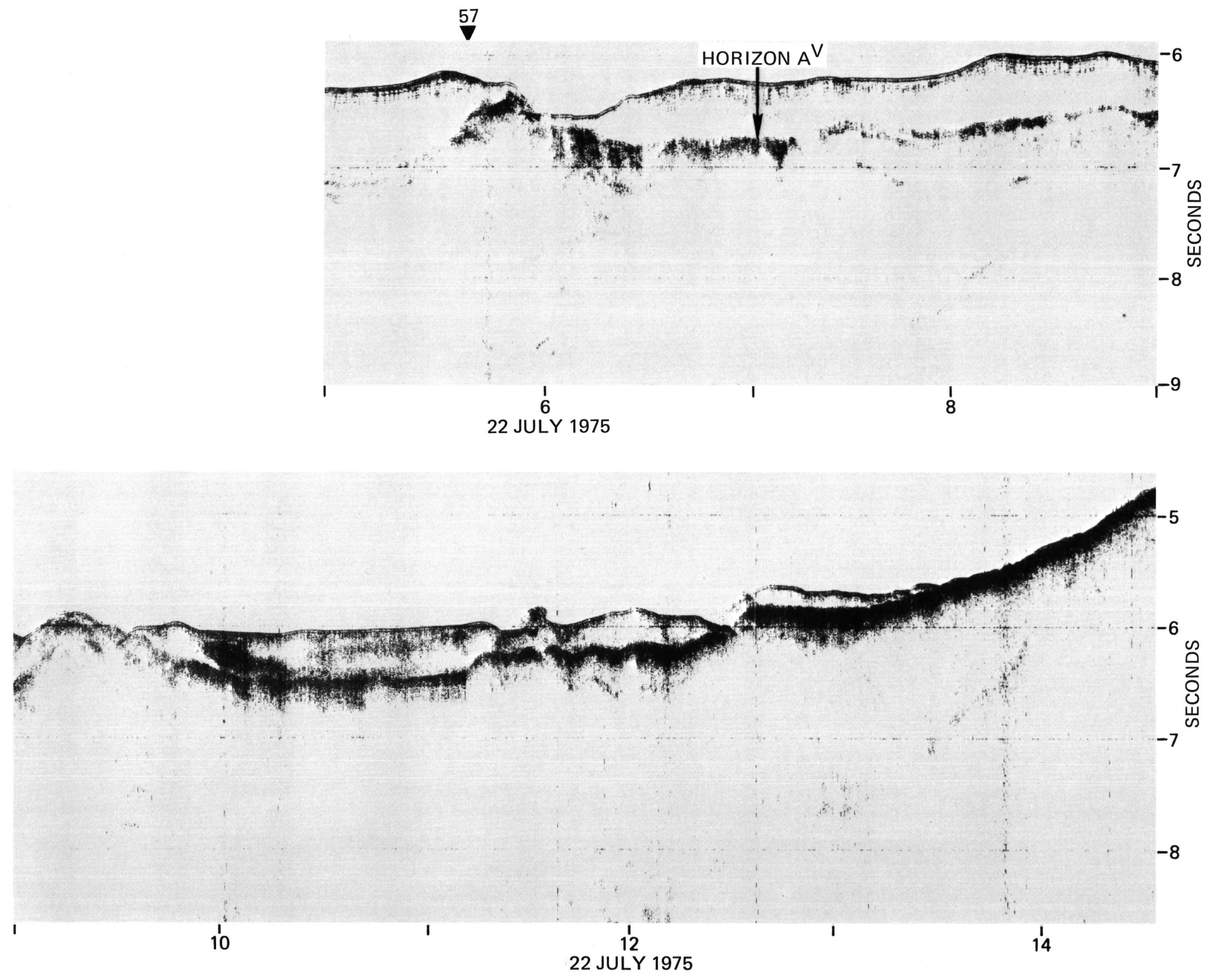

Figure 2. (Continued). 


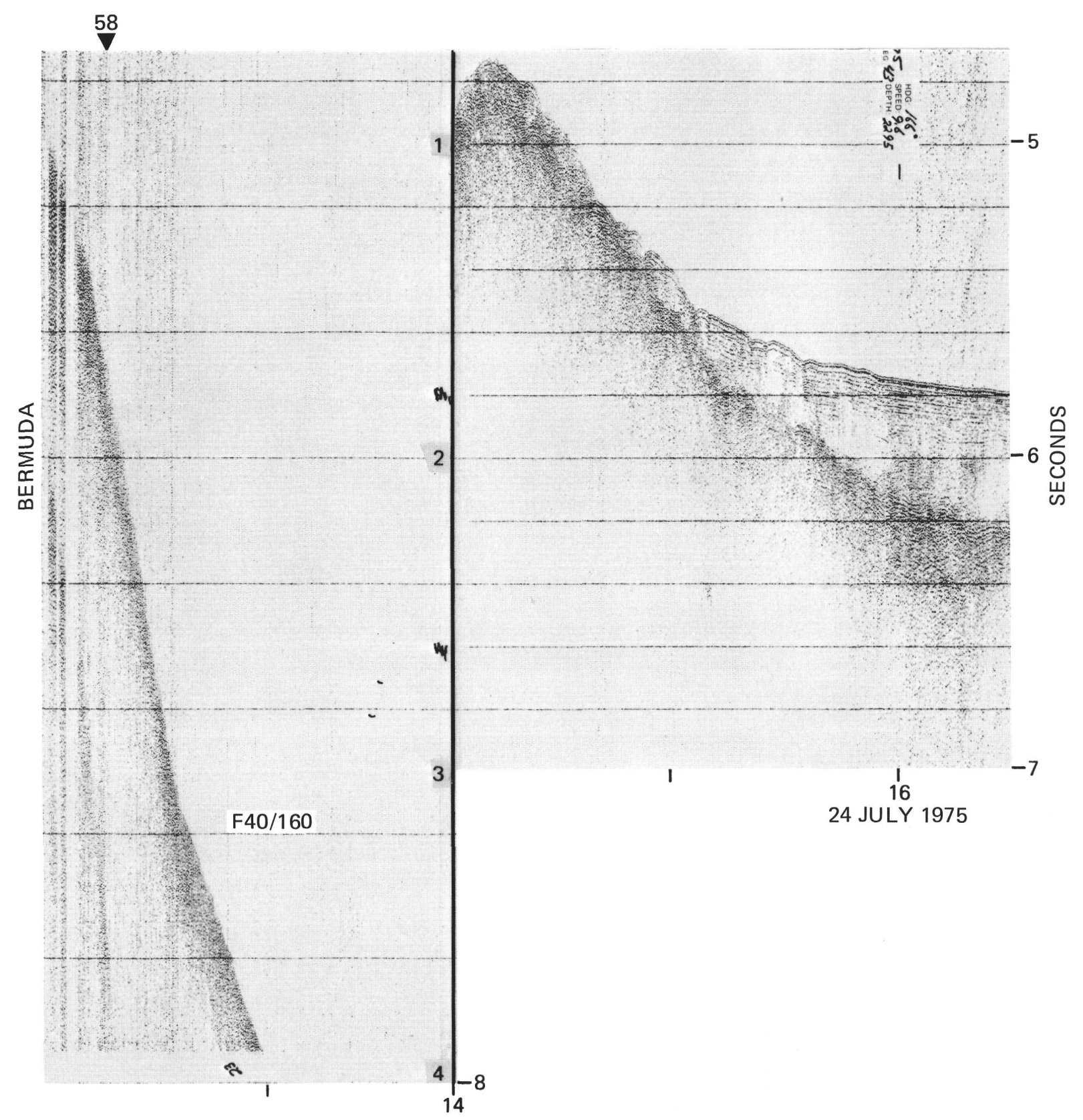

Figure 2. (Continued). 
SITE 386

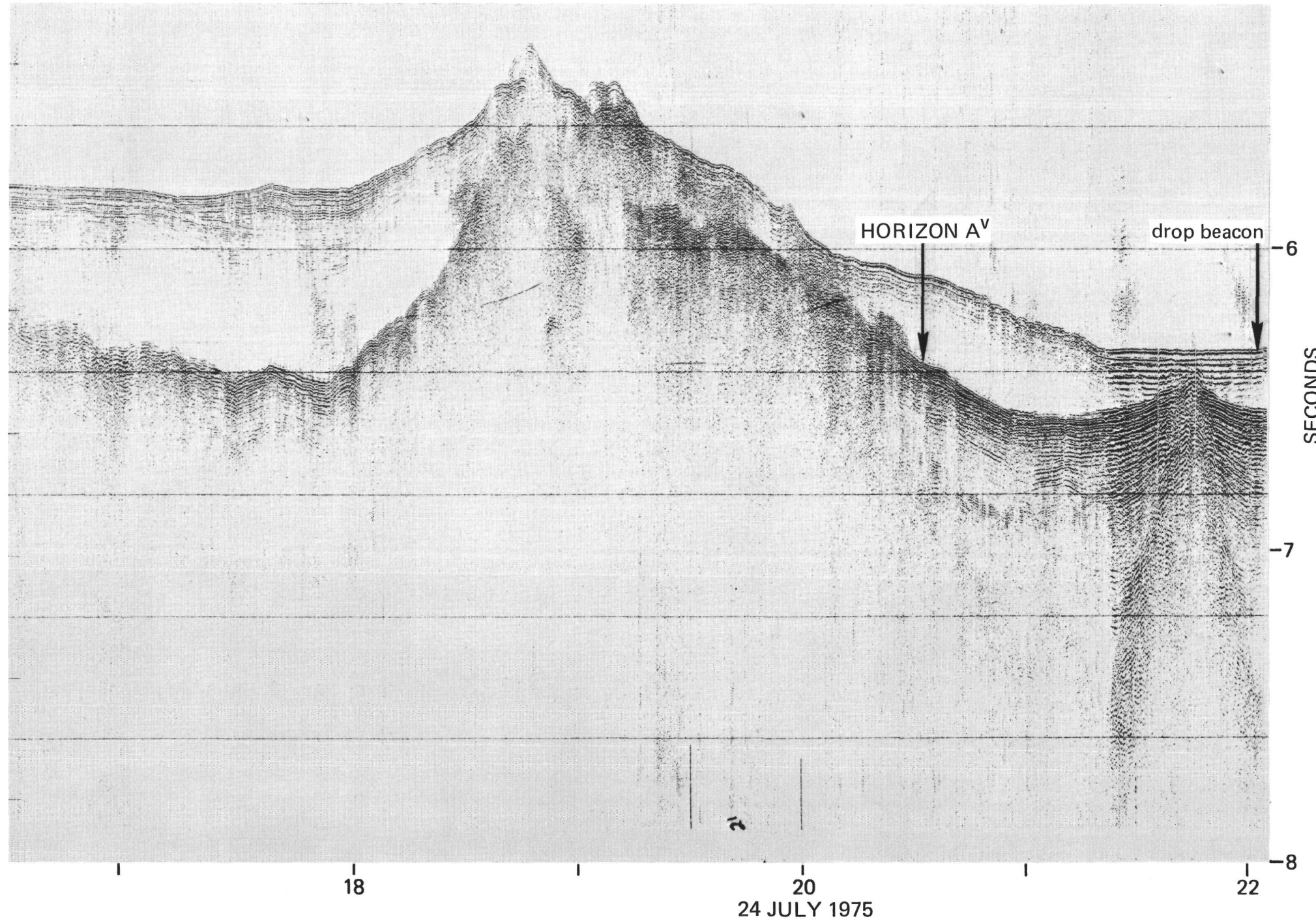

Figure 2. (Continued). 


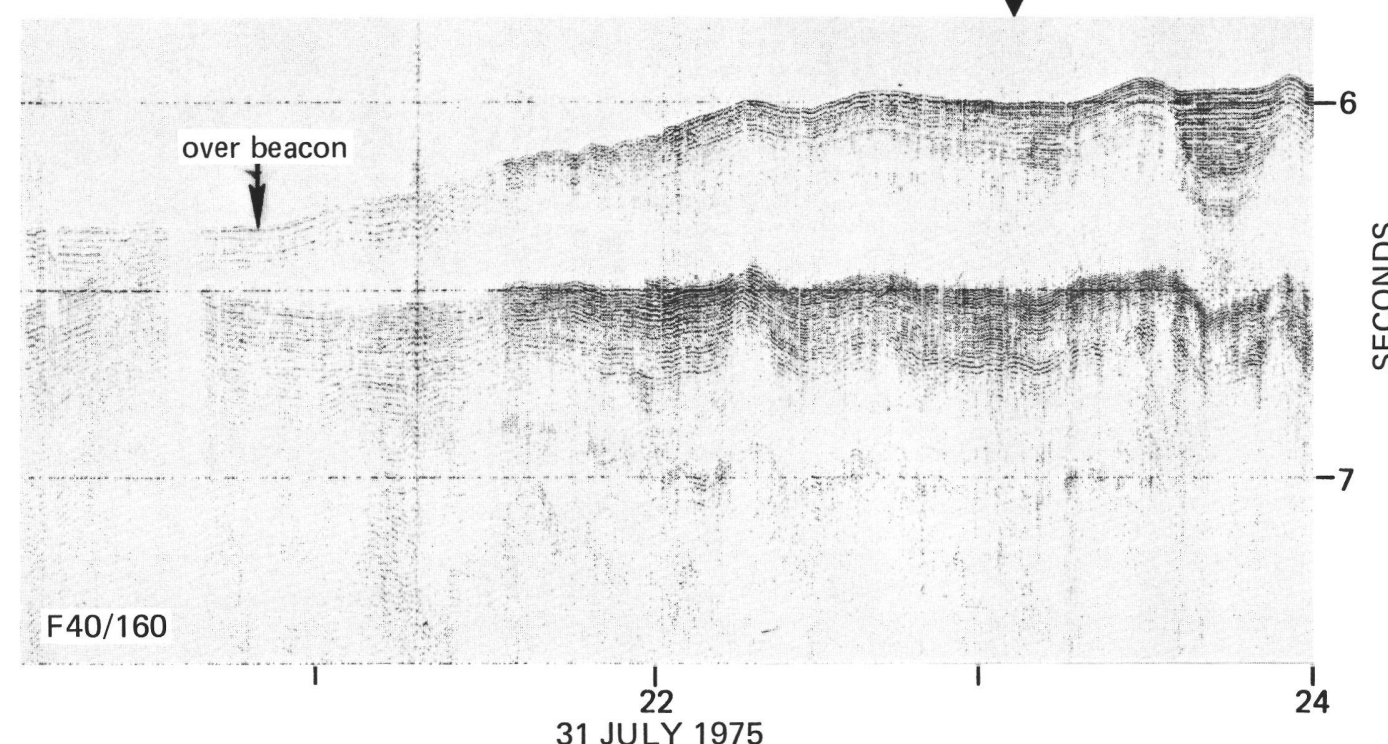

31 JULY 1975

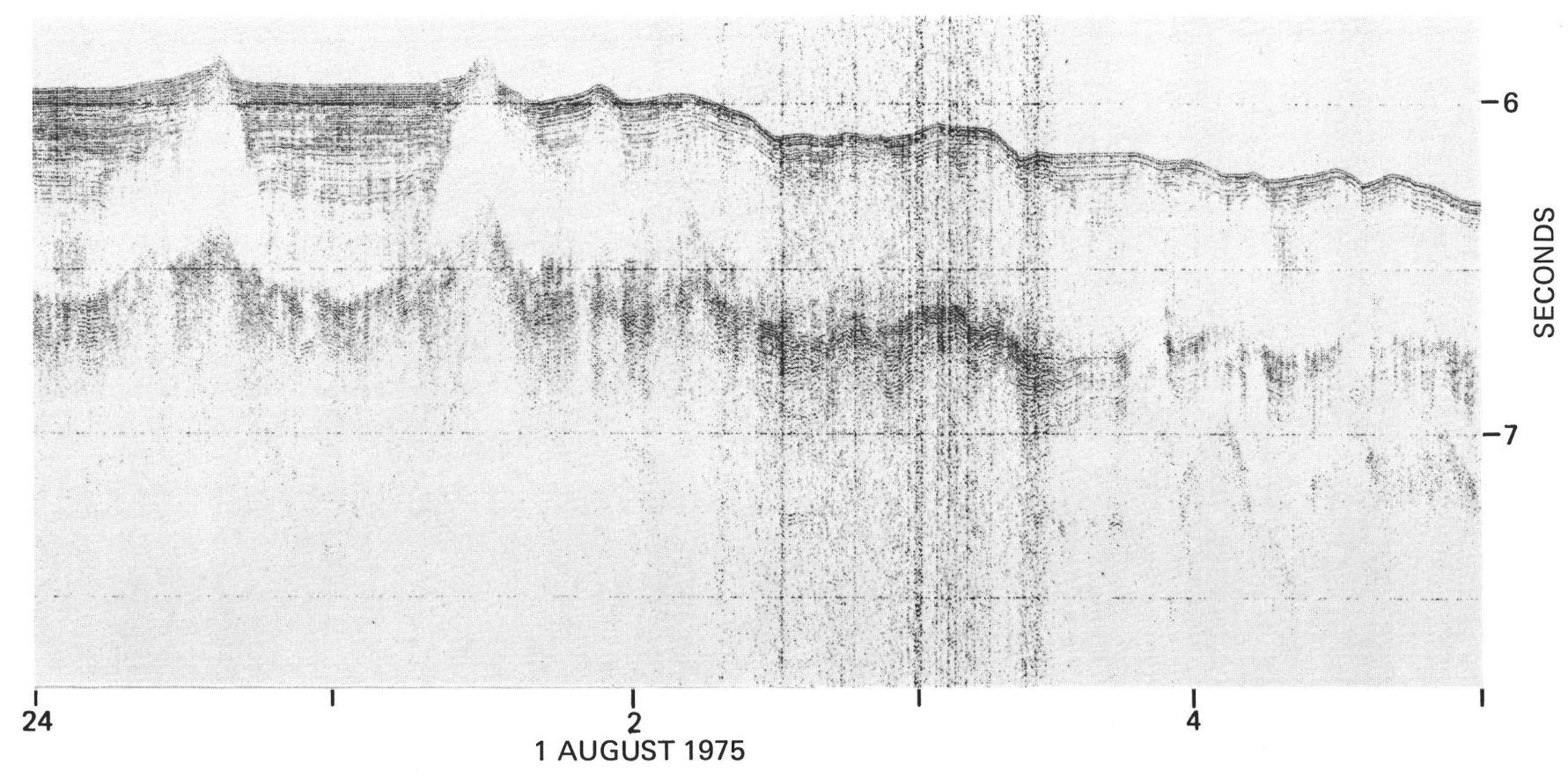

Figure 2. (Continued). 

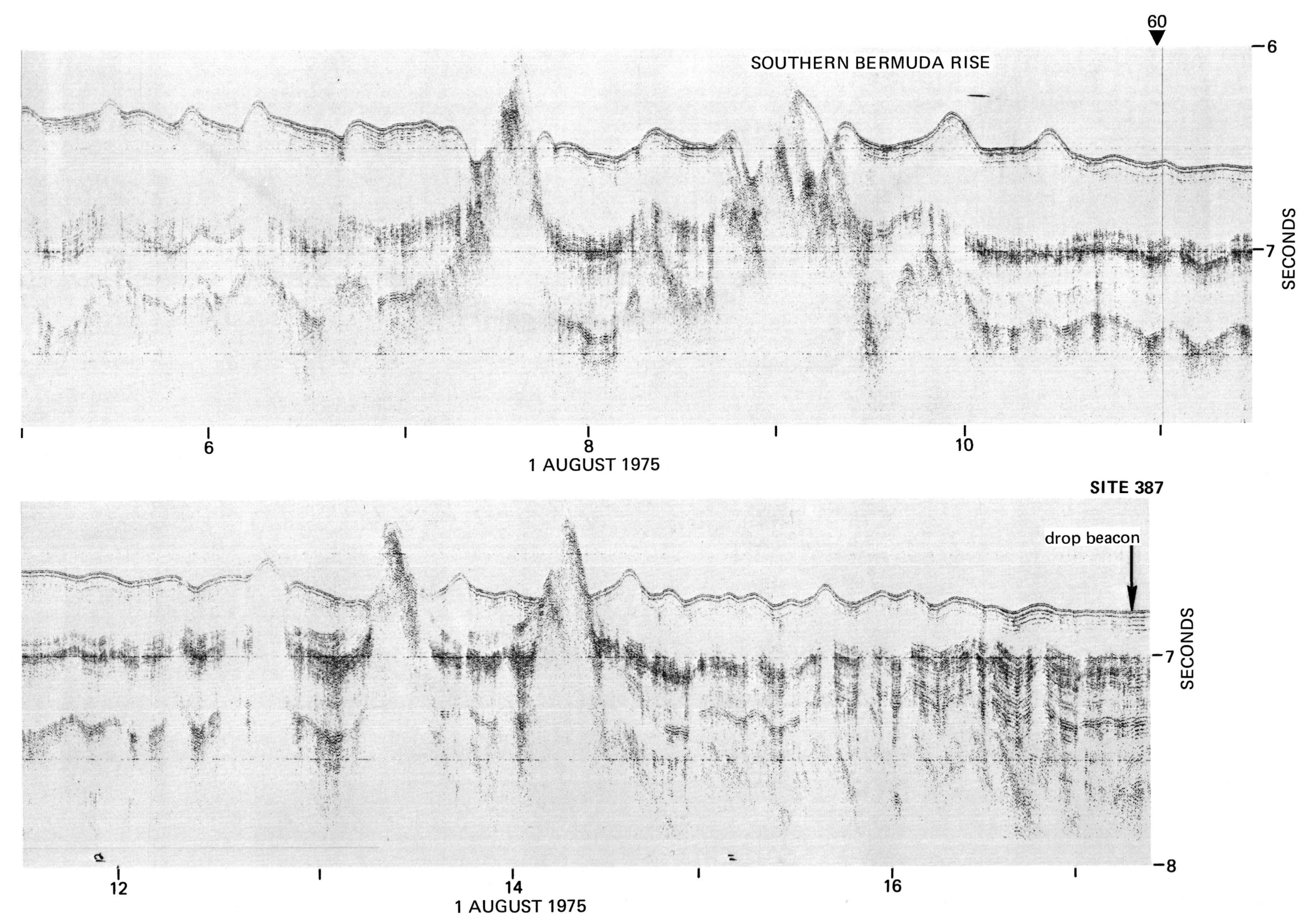


\section{1}

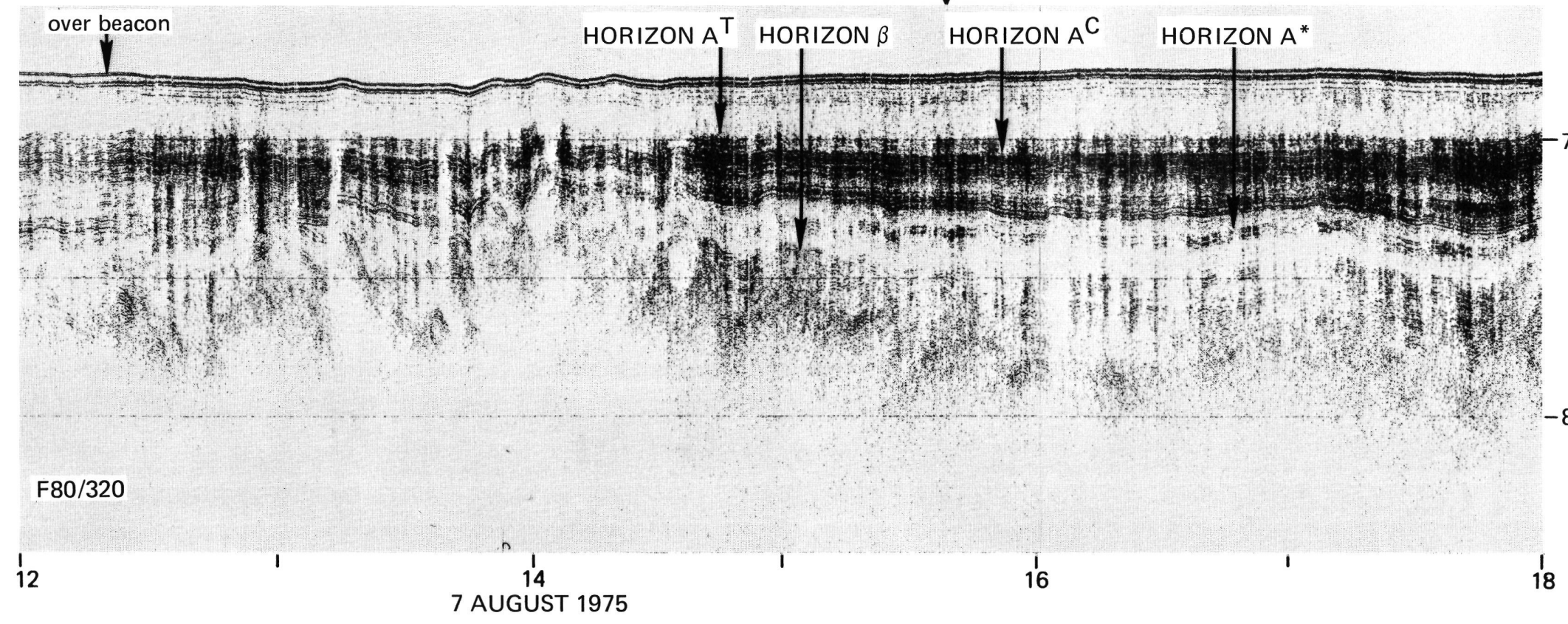

Figure 2. (Continued). 

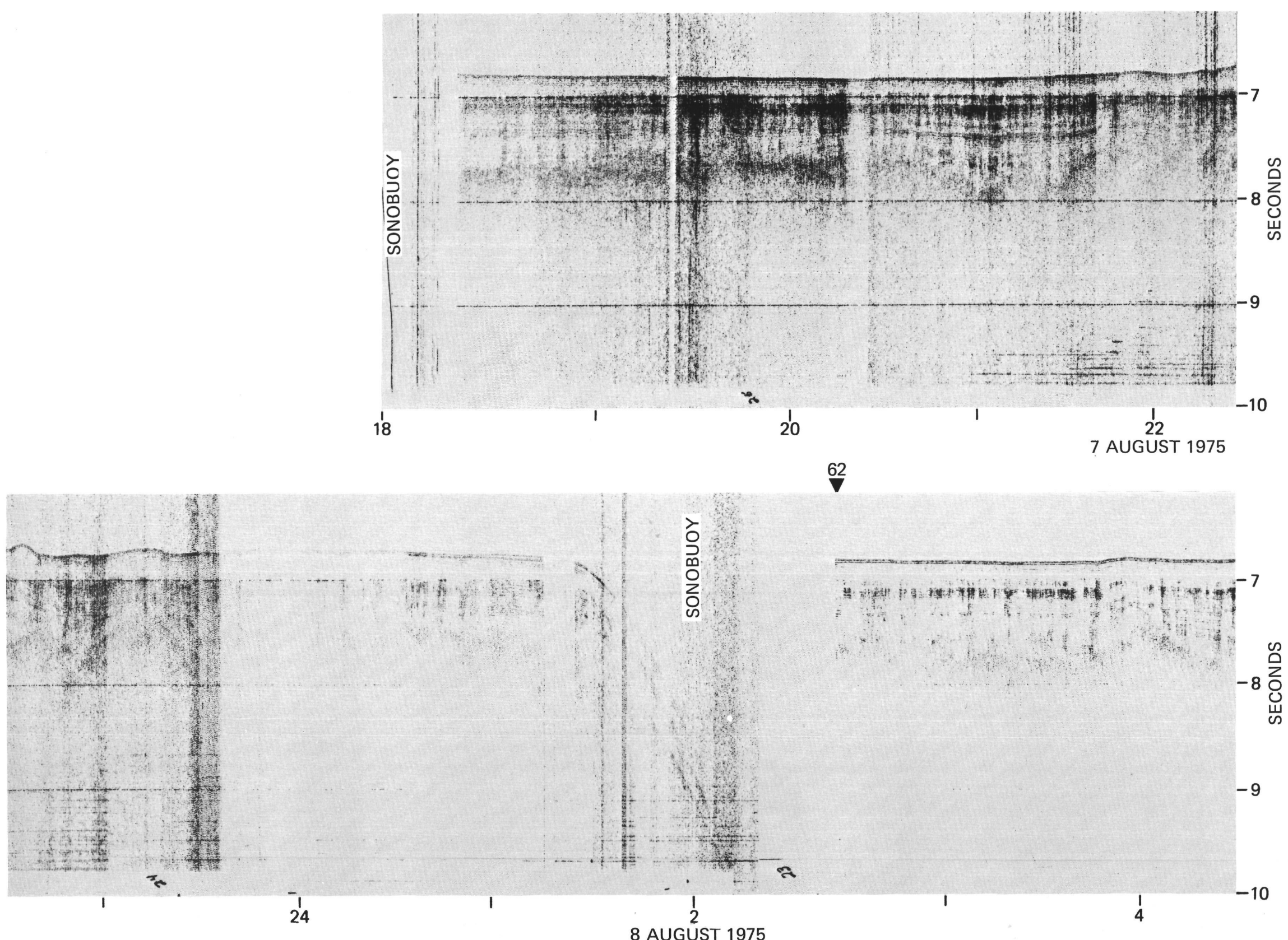

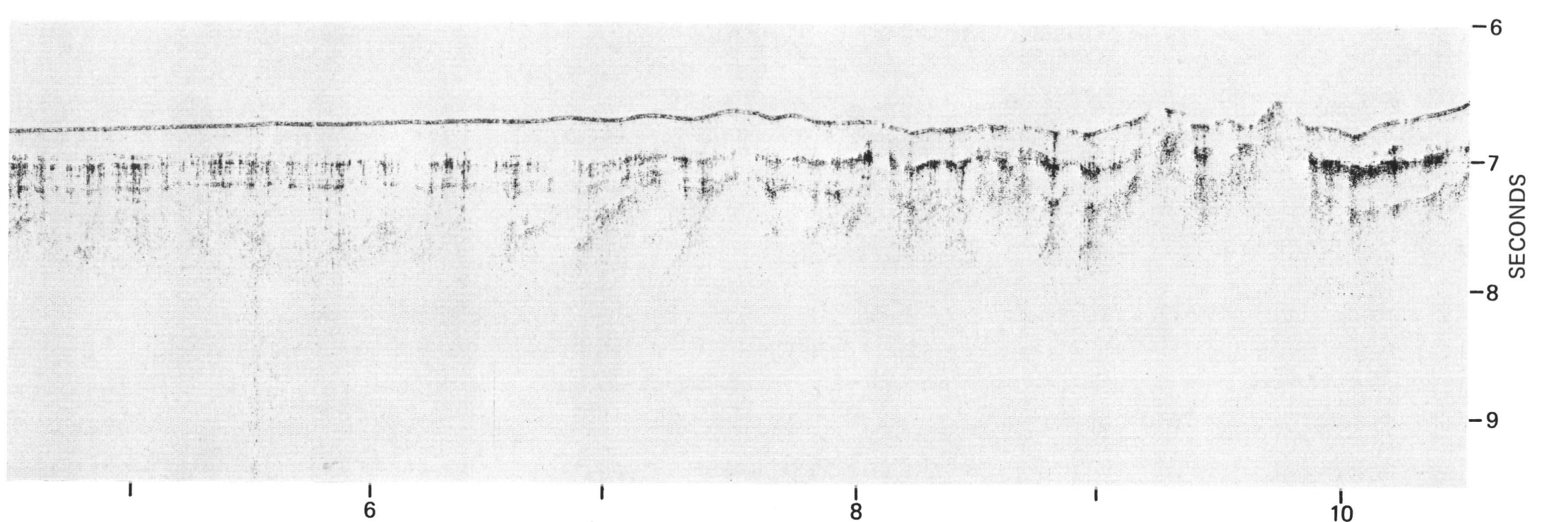

1

8

8 AUGUST 1975

$\sqrt[63]{7}$

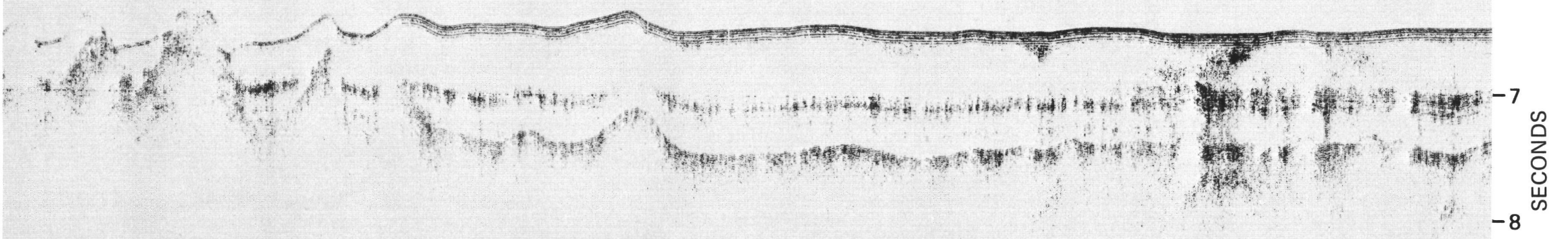

।

12

$\begin{array}{lll}1 & 1 & 1\end{array}$

14

16

8 AUGUST 1975

Figure 2. (Continued). 


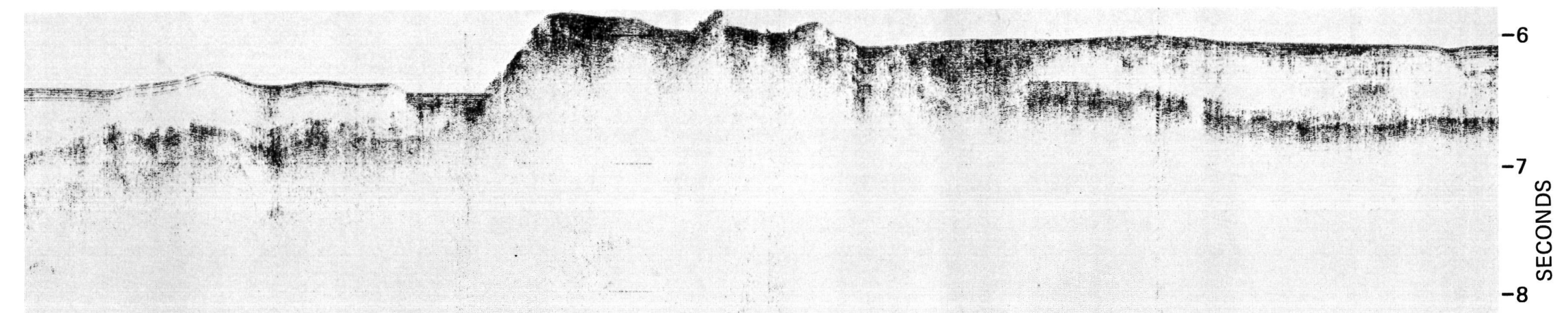

20

22

12

8 AUGUST 1975

64

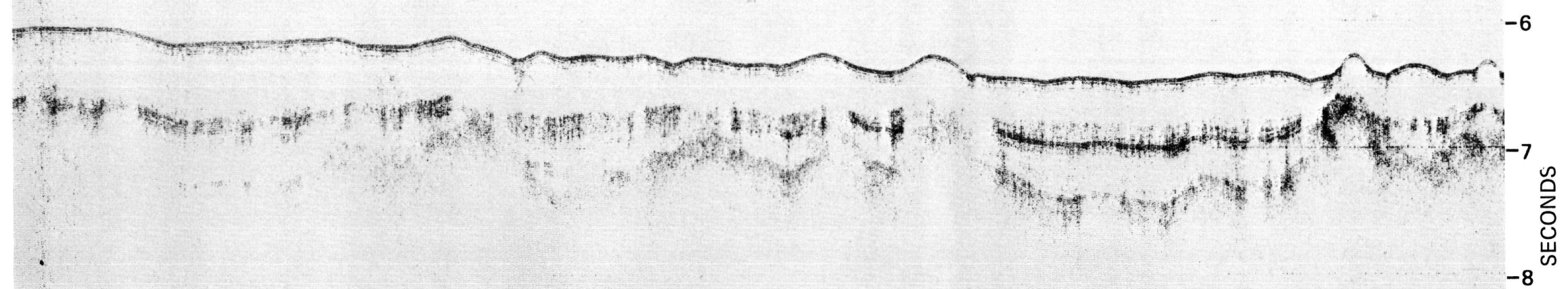




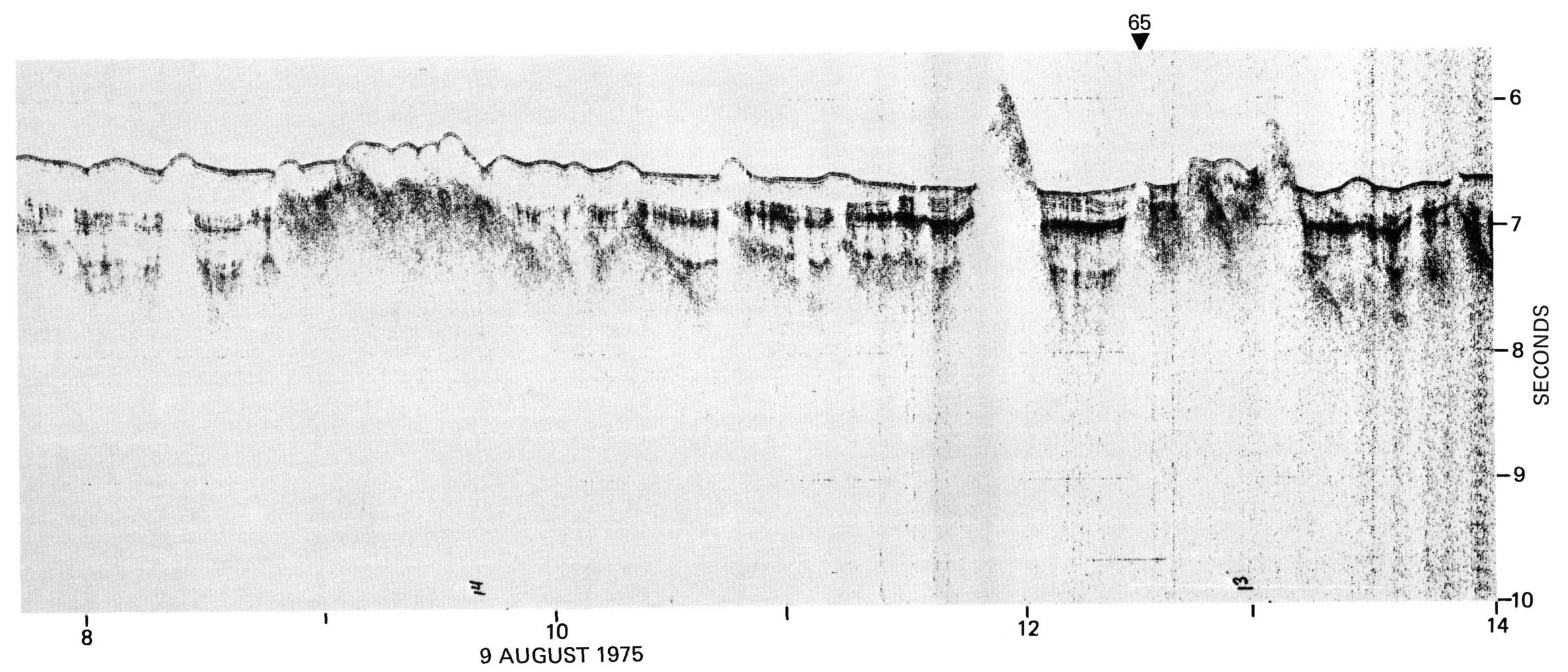

Figure 2. (Continued). 
NEAR SITE 387
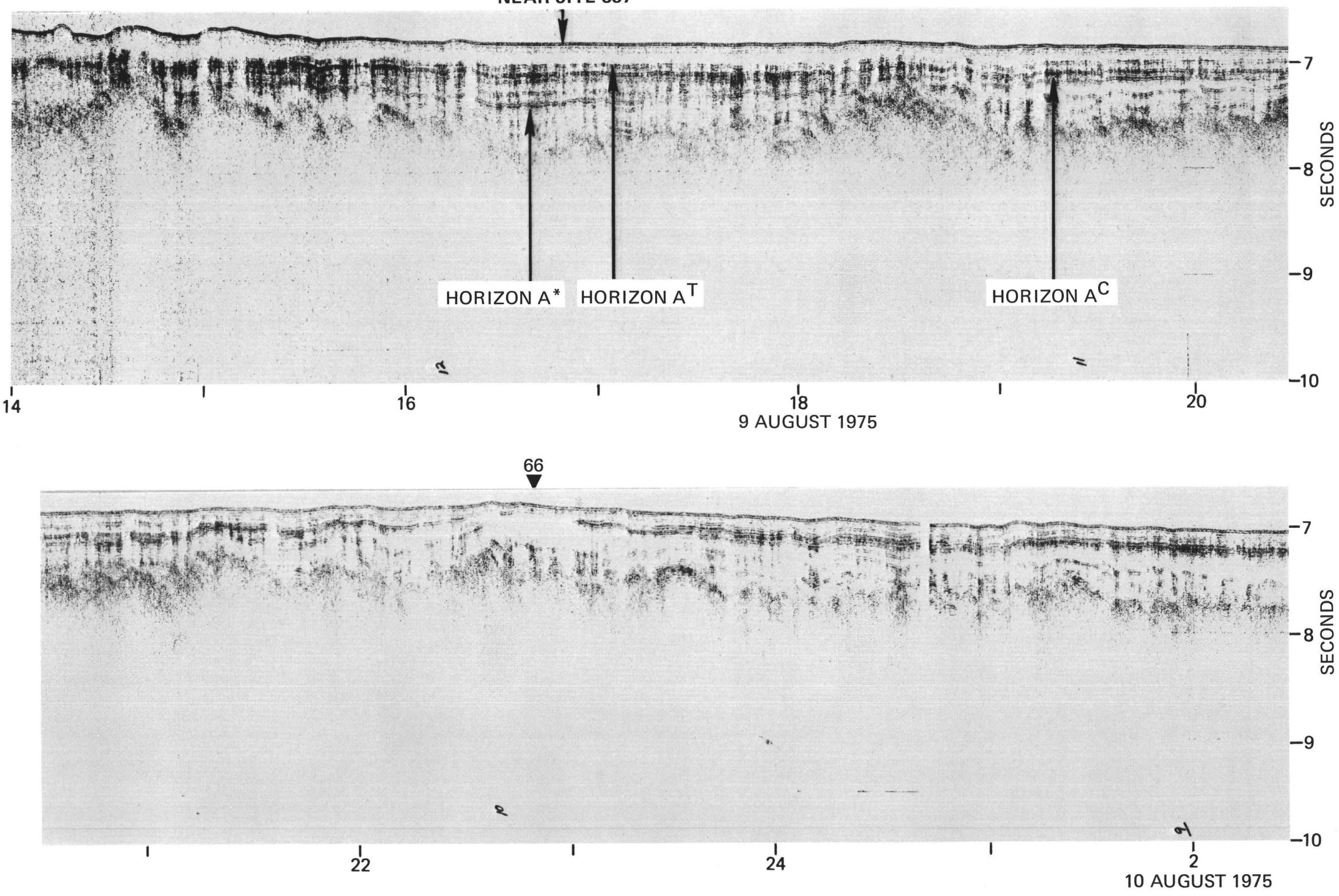

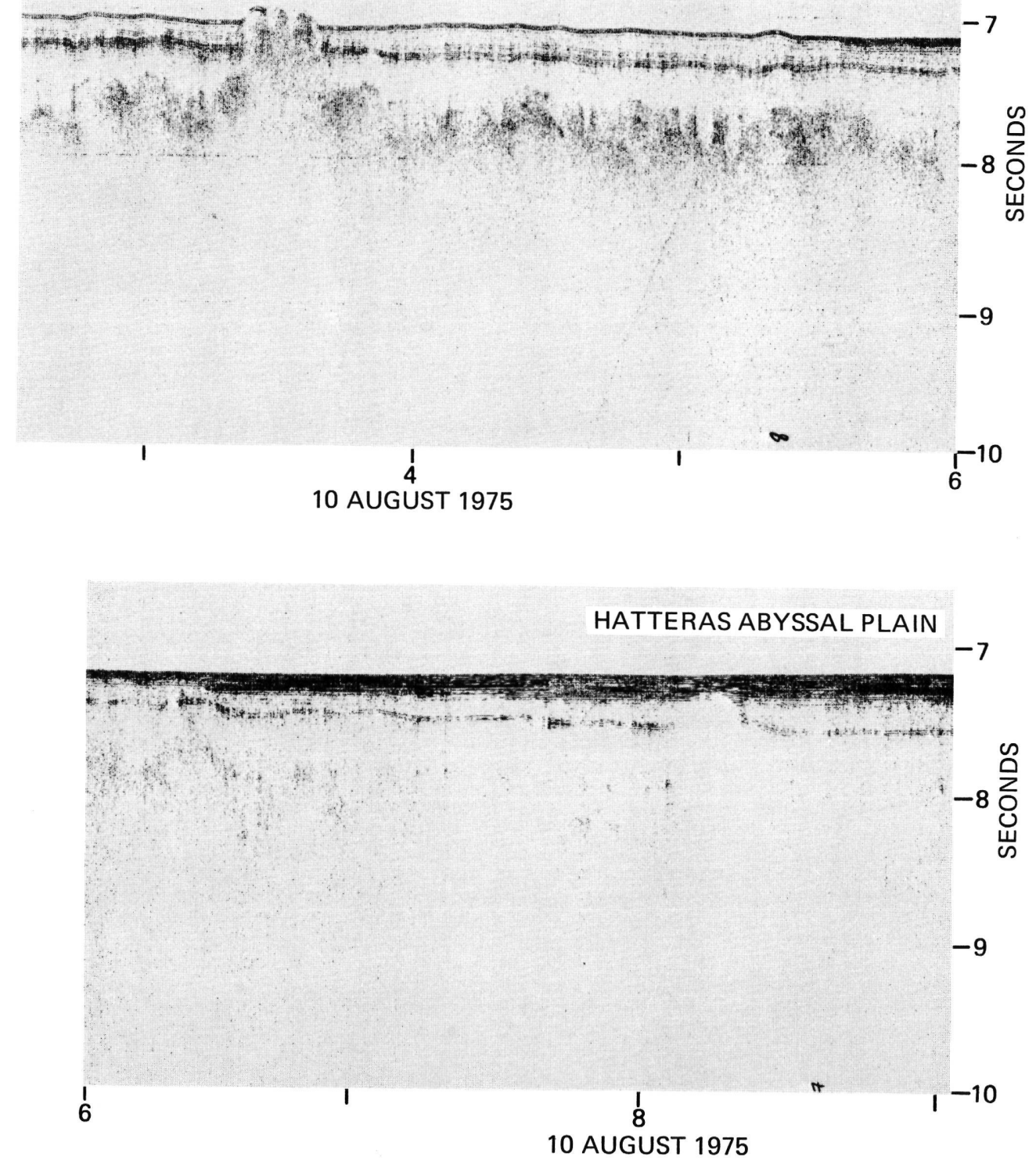

Figure 2. (Continued). 

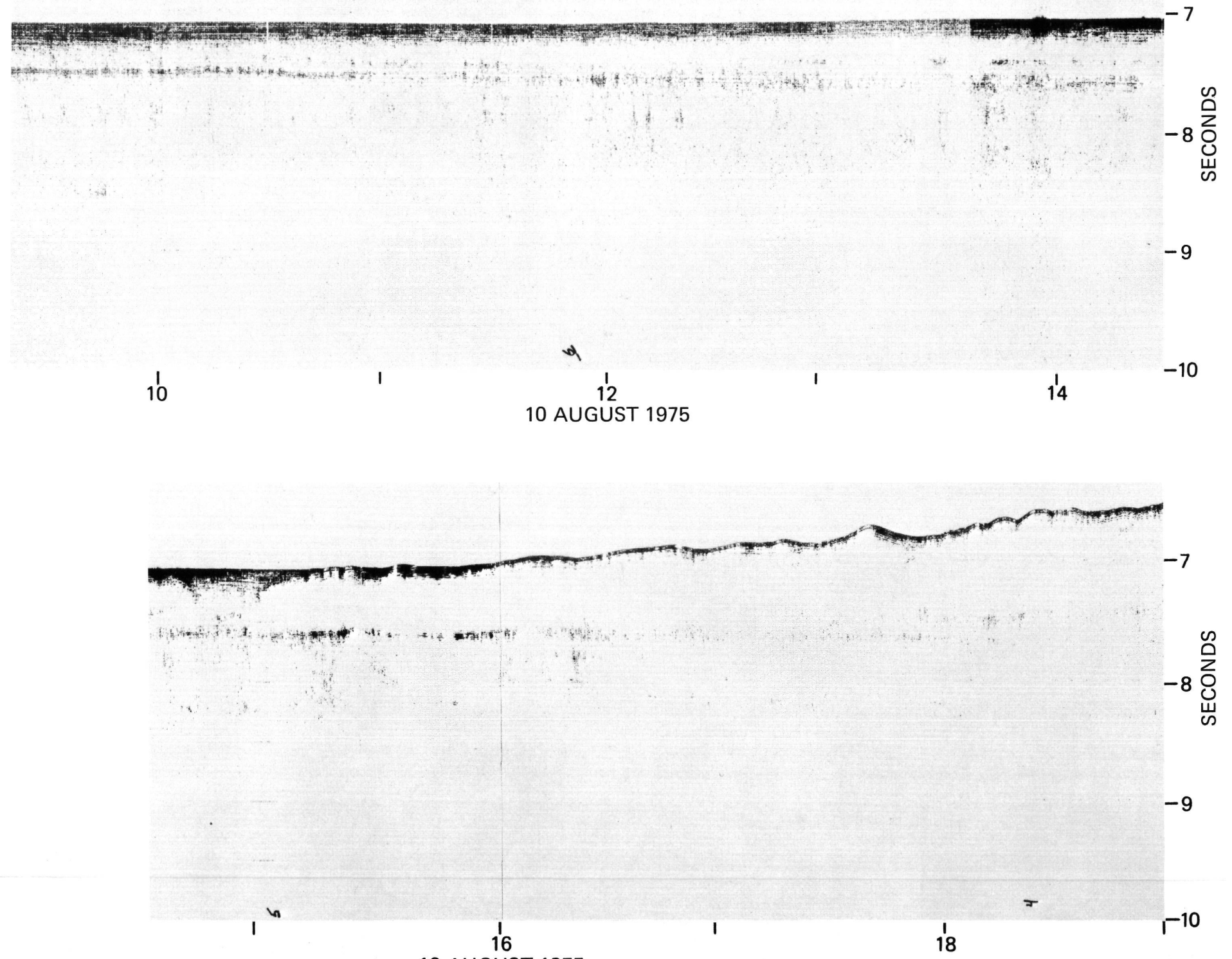

10 AUGUST 1975 


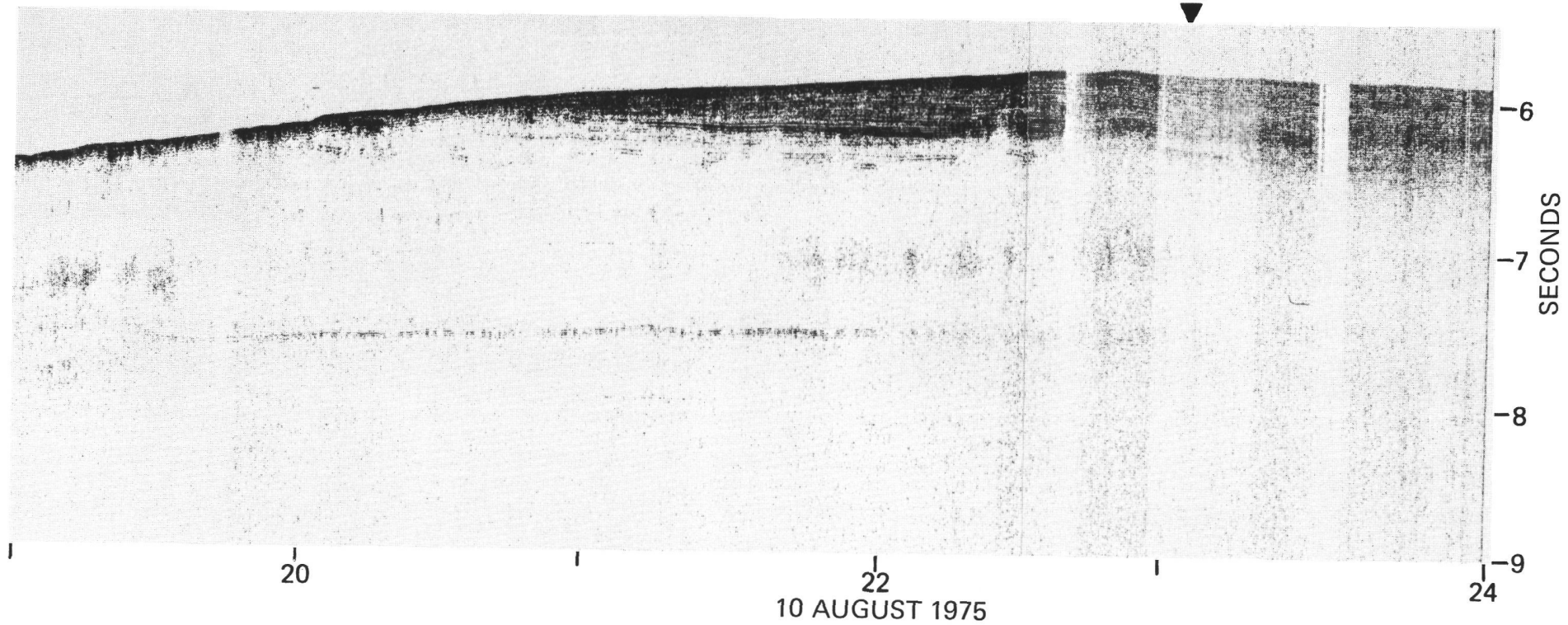

\section{AUGUST 197}

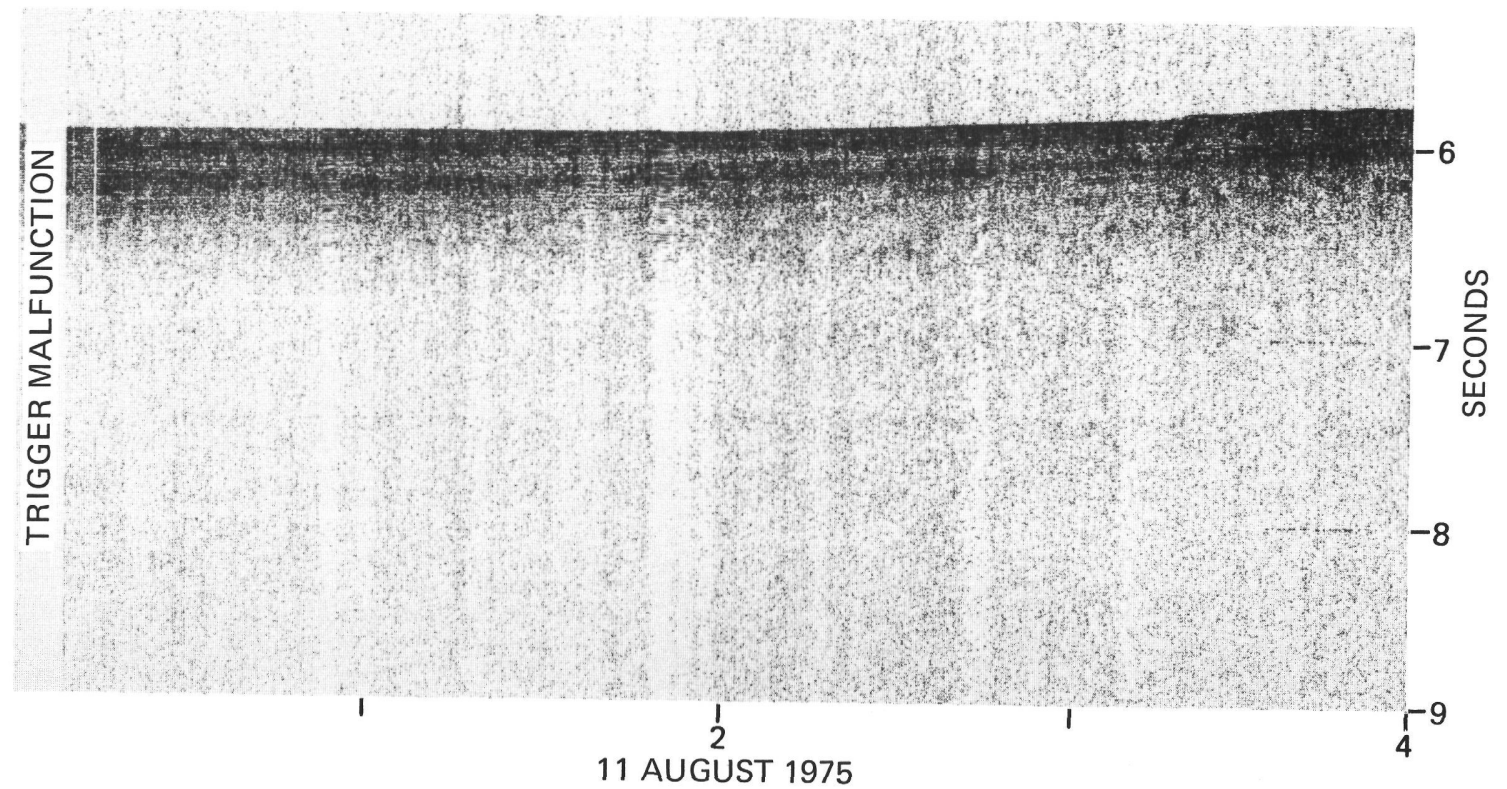

Figure 2. (Continued). 

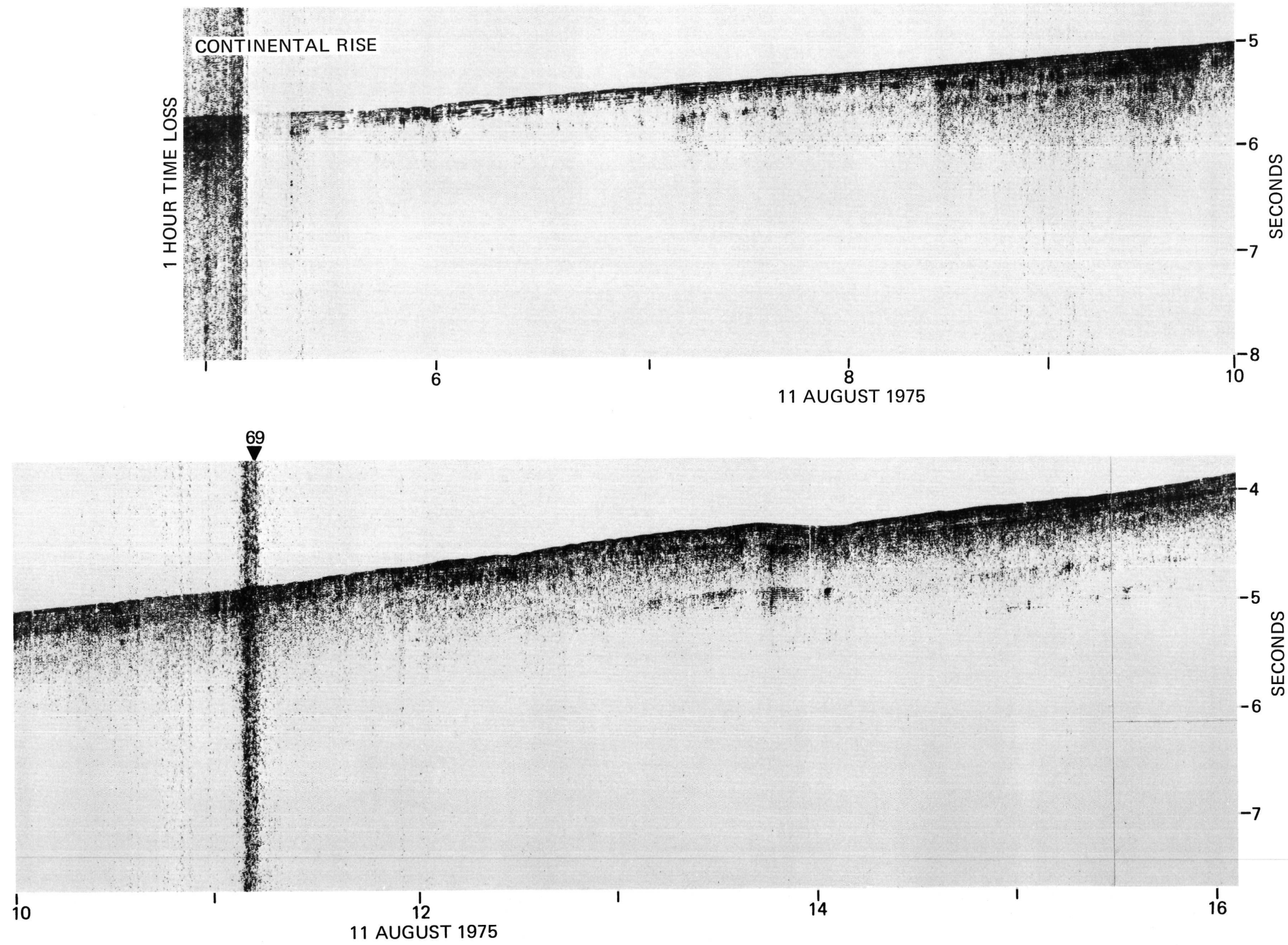


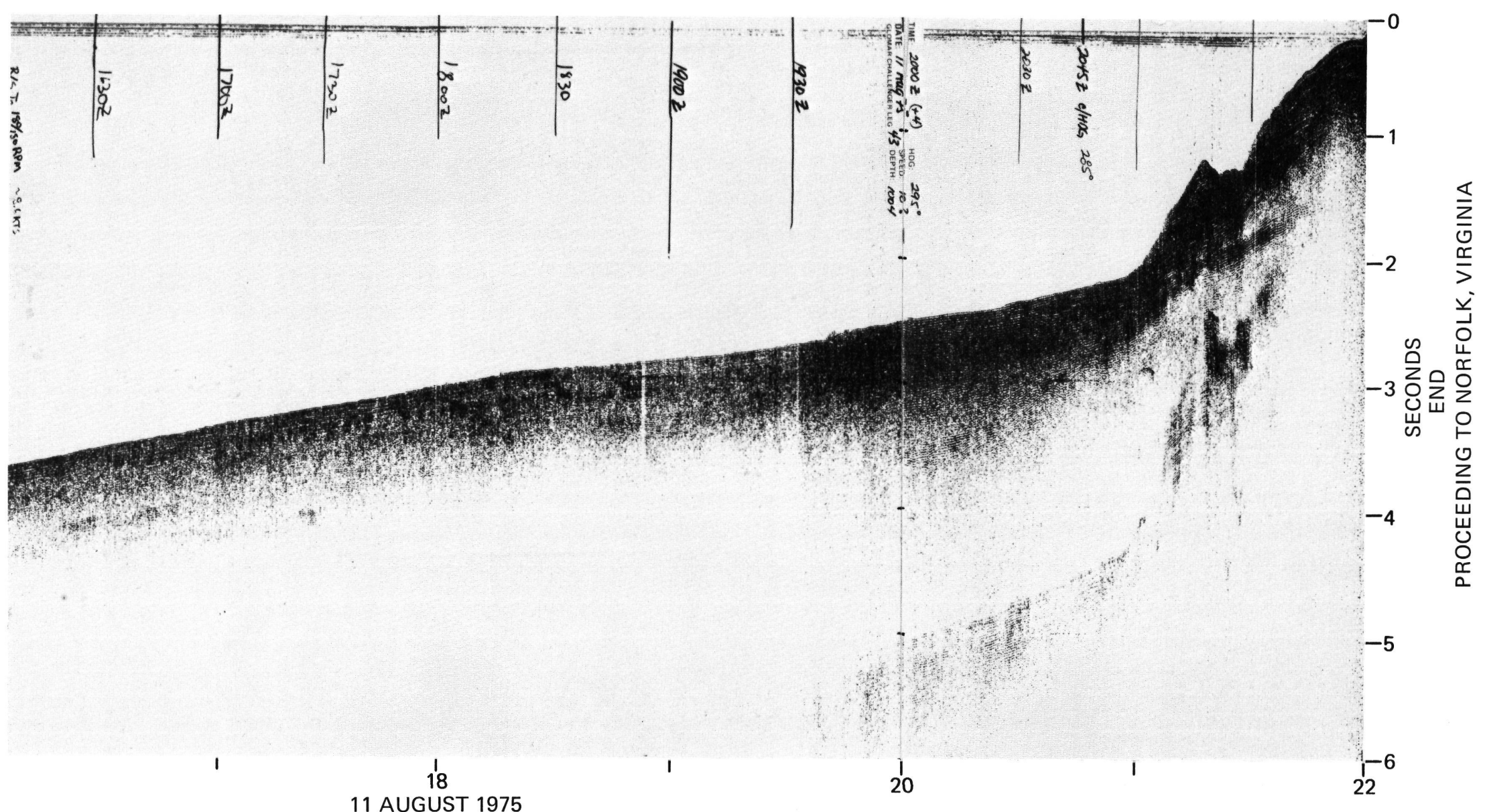

Figure 2. (Continued). 